DESY 17-071

\title{
Exotics: Heavy Pentaquarks and Tetraquarks
}

\author{
Ahmed Ali, ${ }^{1}$ Jens Sören Lange, ${ }^{2}$ and Sheldon Stone ${ }^{3}$ \\ ${ }^{1}$ DESY Theory Group, Notkestrasse 85, D-22607 Hamburg, Germany \\ ${ }^{2}$ Justus-Liebig-Universität Giessen, II. Physikalisches Institut, Heinrich-Buff-Ring 16, \\ D-35392 Giessen, Germany \\ ${ }^{3}$ Physics Department, 201 Physics Building, Syracuse University, Syracuse, NY 13244-1130, USA
}

June 5, 2017

\begin{abstract}
For many decades after the invention of the quark model in 1964 there was no evidence that hadrons are formed from anything other than the simplest pairings of quarks and antiquarks, mesons being formed of a quark-antiquark pair and baryons from three quarks. In the last decade, however, in an explosion of data from both $e^{+} e^{-}$and hadron colliders, there are many recently observed states that do not fit into this picture. These new particles are called generically "exotics". They can be either mesons or baryons. Remarkably, they all decay into at least one meson formed of either a $c \bar{c}$ or $b \bar{b}$ pair. In this review, after the introduction, we explore each of these new discoveries in detail first from an experimental point of view, then subsequently give a theoretical discussion. These exotics can be explained if the new mesons contain two-quarks and twoantiquarks (tetraquarks), while the baryons contain four-quarks plus an antiquark (pentquarks). The theoretical explanations for these states take three divergent tracks: tightly bound objects, just as in the case of normal hadrons, but with more constituents, or loosely bound "molecules" similar to the deuteron, but formed from two mesons, or a meson or baryon, or more wistfully, they are not multiquark states but appear due to kinematic effects caused by different rescatterings of virtual particles; most of these models have all been post-dictions. Both the tightly and loosely bound models predict the masses and related quantum numbers of new, as yet undiscovered states. Thus, future experimental discoveries are needed along with theoretical advances to elucidate the structure of these new exotic states.
\end{abstract}

\section{Contents}

1 Introduction

2 Theoretical techniques for quarkonia and exotic hadrons spectroscopy 6

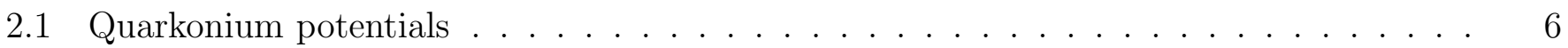

2.2 Lattice simulations . . . . . . . . . . . . . . . . . . . . . 7

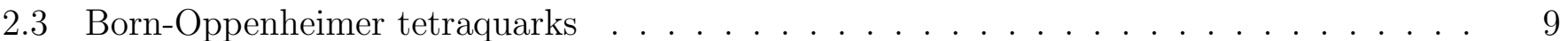

2.4 Doubly heavy tetraquarks on the lattice $\ldots \ldots \ldots \ldots \ldots \ldots$ 
3 Experimental evidence for pentaquarks 11

3.1 History . . . . . . . . . . . . . . . . . . . . . . . . . . . 11

3.2 The LHCb observation of pentaquark charmonium states . . . . . . . . . . . 12

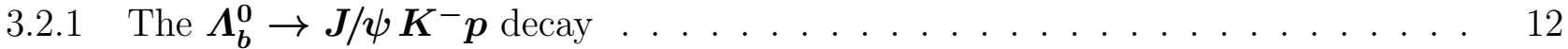

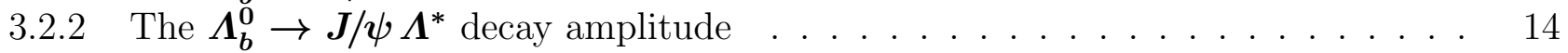

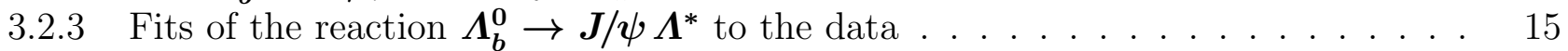

3.2 .4 The matrix element for $\boldsymbol{\Lambda}_{b}^{\mathbf{0}} \rightarrow \boldsymbol{J} / \boldsymbol{\psi} \boldsymbol{P}_{\boldsymbol{c}}^{+} \boldsymbol{K}^{-}$decay $\ldots \ldots \ldots \ldots \ldots \ldots$

3.2.5 Amplitude fits of $\boldsymbol{\Lambda}_{b}^{\mathbf{0}} \rightarrow \boldsymbol{J} / \boldsymbol{\psi} \boldsymbol{K}^{-} \boldsymbol{p}$ allowing $\boldsymbol{P}_{\boldsymbol{c}}^{+}$states $\ldots \ldots \ldots \ldots \ldots$

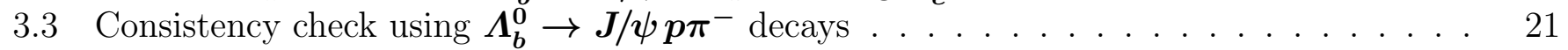

3.4 Independent confirmation of the $\boldsymbol{P}_{\boldsymbol{c}}(\mathbf{4 4 5 0})^{+}$using a model independent $\boldsymbol{\Lambda}^{*}$ description 25

3.5 Impending studies using photoproduction of $\boldsymbol{J} / \boldsymbol{\psi} \boldsymbol{p}$ resonances . . . . . . . . . . 229

4 Experimental evidence for tetraquarks 30

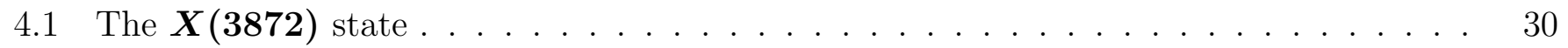

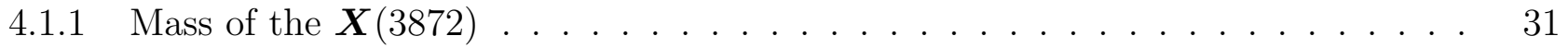

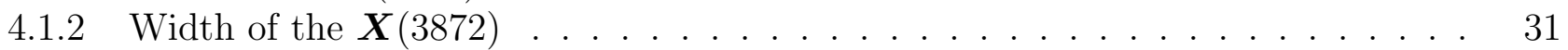

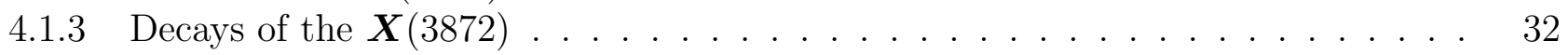

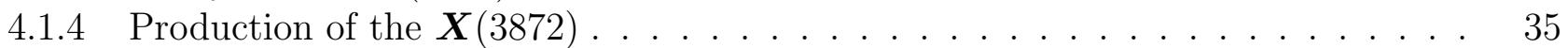

4.1 .5 Interpretation of the $\boldsymbol{X}(3872) \ldots \ldots \ldots \ldots \ldots \ldots \ldots$

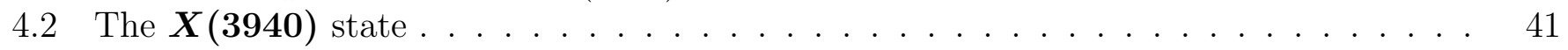

$4.3 \quad \boldsymbol{Y}$ states . . . . . . . . . . . . . . . . . . . . . . . . . . 43

$4.4 \boldsymbol{Z}$ states of type I in the charmonium mass regime . . . . . . . . . . 47

$4.5 Z$ states of type II in the charmonium mass regime . . . . . . . . . . 50

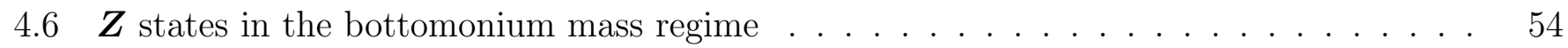

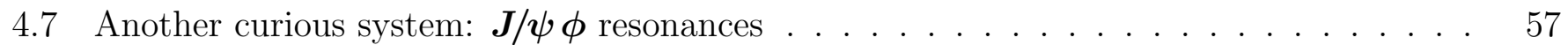

5 Theoretical models for tetraquarks 61

5.1 Tetraquarks as cusps . . . . . . . . . . . . . . . . . . . 62

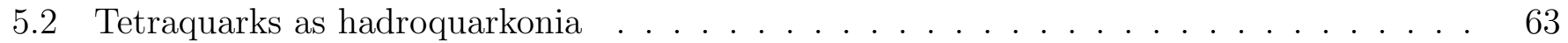

5.3 Tetraquarks as hybrids . . . . . . . . . . . . . . . . . . . . . 64

5.4 Tetraquarks as hadron molecules . . . . . . . . . . . . . . . 64 64

5.5 Tetraquarks as compact diquark-antidiquark mesons . . . . . . . . . . . . 65 65

5.6 The diquark model . . . . . . . . . . . . . . . . . . . . . . . . 66 66

5.7 Non-relativistic Hamiltonian for tetraquarks with hidden charm . . . . . . . . . . 67

5.8 Low-lying $\boldsymbol{S}$ and $\boldsymbol{P}$-wave tetraquark states in the $\boldsymbol{c} \overline{\boldsymbol{c}}$ and $\boldsymbol{b} \overline{\boldsymbol{b}}$ sectors . . . . . . 68

5.9 Heavy-Quark-Spin Flip in $\boldsymbol{\Upsilon}(\mathbf{1 0 8 9 0}) \rightarrow \boldsymbol{h}_{\boldsymbol{b}}(\mathbf{1} \boldsymbol{P}, \mathbf{2} \boldsymbol{P}) \boldsymbol{\pi} \boldsymbol{\pi} \ldots \ldots \ldots \ldots \ldots$

5.10 Drell-Yan mechanism for vector exotica production at the LHC and Tevatron . . . . . 755

6 Theoretical models for pentaquarks

6.1 Pentaquarks as rescattering-induced kinematic effects $\ldots \ldots \ldots \ldots \ldots \ldots$

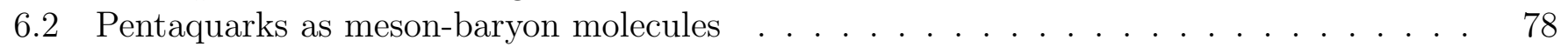

6.3 Pentaquarks in the compact diquark models . . . . . . . . . . . . . . . 78

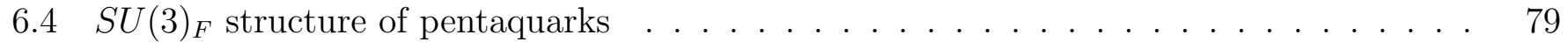

6.5 An effective Hamiltonian for the hidden charm pentaquarks . . . . . . . . . . . . . 80

$6.6 \boldsymbol{b}$-baryon decays to pentaquarks and heavy quark symmetry $\ldots \ldots \ldots \ldots \ldots$

6.7 Weak decays with $\mathbb{P}$ in decuplet representation . . . . . . . . . . . . 83

7 Summary 86

References 88 


\section{Introduction}

When the two $B$ factory experiments $B A B A R$ and Belle were proposed, well over a quarter century ago, their main goal was to measure the matter-antimatter asymmetry in the $B$-meson sector 11 This goal they indeed accomplished very well, together with other related key measurements in flavor physics, which greatly helped in testing the charged current weak interactions in the standard model [1]. While in depth studies of hadronic structure was not on the agenda of these experiments, they also succeeded in uncovering a new facet of QCD. The discovery of the exotic hadron $X(3872)$ in 2003 in the decays $B^{0} \rightarrow J / \psi \pi^{+} \pi^{-} K^{-}$by Belle [2] came as a complete surprise..$^{2}$ Confirmation soon thereafter by BABAR [3, 4], CDF II [5 7], D0 [8], and later by the LHCb [9] and CMS [10], established the $X(3872)$ as a genuine resonance, as opposed to a threshold effect (called a "cusp"). Its discovery turned out to be the harbinger of a new direction in hadron physics. Thus, out of the debris of $B$-meson decays emerged a second layer of strongly interacting particles called tetraquarks (four-quark states), all containing a $c \bar{c}$ quark pair. Likewise, in the decays of the $\Lambda_{b}$-baryon, LHCb later established the existence of two pentaquark states $P_{c}^{+}(4380)$ and $P_{c}^{+}(4450)$ in the $J / \psi p$ decay mode [11]. As the minimum valence quark content of such a state is $c \bar{c} u u d$, the newly discovered baryons have five quarks. Meanwhile, well over two dozen exotic hadrons have found entries in the Particle Data Group [12]. Tentatively called $X, Y, Z$ and $P_{c}$, they have various $J^{P C}$ quantum numbers, and come both as charged and neutrals, as discussed in detail in this review and elsewhere $[13 \sqrt{15}]$. At least two $b \bar{b}$ counterparts of the $c \bar{c}$ states, $Z_{b}^{+}(10610)$ and $Z_{b}^{+}(10650)$, have also been reported by Belle [16]. Their valence quark content is $b \bar{b} u \bar{d}$. A closely related question is whether tetraquarks and pentaquarks also come with a single charm or bottom valence quark.

Quarkonium physics is a well-studied system theoretically, having its roots in the non-relativistic quarkonium potential [17, 18]. Its present formulation is in the context of effective field theories based on QCD [19, 20] and lattice-QCD [21 24]. The progress in lattice-based techniques is impressive and allows us to connect the observed hadronic properties with the fundamental parameters in QCD. In particular, hadron spectra below the strong decay thresholds are calculated accurately, and extensive results for multiplets are available. However, so far there are no reliable lattice results for flavor exotic states, though possible $c \bar{c} \bar{d} u$ candidates with $J^{P C}=1^{+-}$have been searched for in the vicinity of the $D \bar{D}^{*}$ threshold, where the $Z_{c}^{+}(3900)$ is found experimentally. No $c \bar{c} s \bar{s}$ resonance has been found in the $J / \psi \phi$ scattering on the lattice, though their experimental evidence has mounted, and no $c c \bar{d} \bar{u}$ bound state is found in the $D D^{*}$ scattering either [25]. We briefly review the current lattice simulations in section 2, but we shall be mostly using the available phenomenological approaches for the theoretical discussion of tetraquarks and pentaquarks in this review.

For the spectra of the $c \bar{c}$ and $b \bar{b}$ bound states below their corresponding open flavor thresholds, $D \bar{D}$ and $B \bar{B}$, potential models are reliable, as they successfully reproduce the observed $1 \mathrm{~S}, 2 \mathrm{~S}$, and $1 \mathrm{P}$ states, in the $c \bar{c}$-, and many more higher states in the $b \bar{b}$-sector. The most popular of these, the Cornell potential 17, 18, incorporates a color Coulomb term at short distances and a linear confining term at large distances, with the spin-dependent interquark interaction responsible for the splitting of the states in these multiplets governed by a one-gluon exchange Breit-Fermi Hamiltonian. The quarkoniumpotential-based studies were substantially extended to cover the $c \bar{c}$ and $b \bar{b}$ states above the open flavor thresholds (see, for example, Ref. [26]). The concordance between experiments and such theoretical estimates is remarkable in both the charm and bottom sectors, predicting correctly a large number of observed states. While empirical, these potential-model based studies provide useful benchmarks for the quarkonia states, as they provide the background upon which exotic particles are to be searched for and characterized.

What are the criteria for an observed hadron to be termed as exotic? The clues to novel features

\footnotetext{
${ }^{1}$ They followed the exploratory work done by the ARGUS and CLEO experiments operating in the $\Upsilon$ resonance region.

${ }^{2}$ Mention of a specific decay mode implies use of the charge conjugate mode as well.
} 
in QCD may come in a number of ways which we discuss here briefly. For example, the exotic hadrons may have unconventional $J^{P C}$ quantum numbers, such as having $J^{P C}=0^{--}, 0^{+-}, 1^{+-}$, which are not allowed for non-relativistic $q \bar{q}$ states. Also, more hadrons with the same $J^{P C}$ quantum numbers could be discovered than are allowed by the quark model counting. The spectrum of the charmonium states around $4 \mathrm{GeV}$ is particularly interesting in this context, as it contains, in addition to the anticipated conventional $c \bar{c}$ states, such as $\psi(3770), \psi(3823)$, the states $X(3872)\left(J^{P C}=1^{++}\right), X(3915)\left(J^{P C}=\right.$ $\left.0^{++}\right)$, the $Z_{c}^{0}(3900)\left(J^{P C}=1^{+-}\right)$, and the $X(3940)$, whose $J^{P C}$ quantum numbers have yet to be determined. At least some of them are good candidates for non- $q \bar{q}$ states. They may also show sytematic different patterns in their intrinsic properties, such as the spin-spin and spin-orbit interactions, compared to what is known for the quarkonium multiplets. The charged states, such as $Z_{c}^{ \pm}(3900), Z_{c}^{ \pm}(4020)$, $Z_{b}^{ \pm}(10610)$ and $Z_{b}^{ \pm}(10650)$, are manifestly non- $q \bar{q}$ due to the minimum valence quark composition required for their discovery modes. They may also exhibit stark differences in their decay characteristics, such as the total and partial widths as well as in their final state profiles, compared to the other similar and well-understood systems. The dipion invariant mass distributions in $\Upsilon(5 S) \rightarrow \Upsilon(n S) \pi^{+} \pi^{-}$, with $n S=1 S, 2 S, 3 S$, as well as the $\Upsilon(n S) \pi^{ \pm}$invariant mass distribution [27], are two cases in point. Dalitz plot studies in these decays reveal unusual structures in both these channels. The decay rate and dipion mass spectrum in the transition of the lower $\Upsilon$ states, such as $\Upsilon(4 S) \rightarrow \Upsilon(1 S) \pi^{+} \pi^{-}$, on the other hand, are well described by the QCD multipole expansion [28, 29], with no structure seen in the Dalitz distributions, as expected from Zweig-forbidden transitions. In line with this, the partial decay width $\Gamma\left(\Upsilon(4 S) \rightarrow \Upsilon(1 S) \pi^{+} \pi^{-}\right)$as well as $\Gamma\left(\Upsilon(4 S) \rightarrow \Upsilon(2 S) \pi^{+} \pi^{-}\right)$and $\Gamma\left(\Upsilon(n S) \rightarrow \Upsilon(m S) \pi^{+} \pi^{-}\right)$, involving the pairs $(3 S, 1 S),(3 S, 2 S),(2 S, 1 S)$, are all of $\mathrm{O}(1) \mathrm{KeV}$. They are typically two orders of magnitude smaller than the $\Upsilon(5 S) \rightarrow \Upsilon(1 S, 2 S, 3 S) \pi^{+} \pi^{-}$partial decay widths [12]. Likewise, decay rates for the heavy quark spin-flip transitions $\Upsilon(5 S) \rightarrow\left(h_{b}(1 P), h_{b}(2 P)\right) \pi^{+} \pi^{-}$are found experimentally comparable to the heavy quark spin non-flip transitions $\Upsilon(5 S) \rightarrow \Upsilon(n S) \pi^{+} \pi^{-}[30]$ - in apparent violation of the heavy quark symmetry. These are all hints of anomalous phenomena, not predicted by the QCD-based phenomenology of the conventional quarkonium systems, and in all likelihood they point to hitherto unexplored and novel facets of QCD.

The emergence of four-quark (more precisely two quarks and two antiquarks) and five-quark ( four quarks and an antiquark) hadrons has provided new challenges for QCD. As discussed in section 2, it is too early for a definite statement on their properties from lattice QCD, and we review several competing phenomenological models put forward in the literature to accommodate them. They range from the mundane (kinematic artifacts called cusps [31,32]) to compact tetraquarks [33], which consist of a $Q \bar{Q}$ pair and a light quark and an antiquark, bound in a compact color-singlet tetraquark $(Q \bar{Q} q \bar{q})_{1}$. The phenomenolgy of compact tetraquarks is developed in the framework where the structure consists of a color-antitriplet diquark $(Q q)_{\overline{3}}$ and a color-triplet antidiquark $(\bar{Q} \bar{q})_{3}$, bound by a gluon. They are called in the literature interchangably as diquark-onium or simply diquark models. The main idea, going back to the earlier papers by Jaffe [34] and Jaffe and Wilczek [35] is that a tightly bound colored diquark plays a fundamental role in the hadron spectroscopy [36], and possibly beyond [37], such as QCD in high baryon density and color superconductivity. In the context of the $X, Y, Z$ tetraquarks, and the $P_{c}$ pentaquarks, we will follow here mainly the works presented in [38 41]. They will be called diquark models in this review. In between are other constructs, motivated by different theoretical scenarios. The hadroquarkonium models [42, 43] are motivated by the analogy with the hydrogen atom, in that the exotic hadrons have a heavy quarkonium core $\left(J / \psi, \psi^{\prime}, \eta_{c} \ldots\right)$, with light $q \bar{q}$ pair around it. There are other models which are motivated by analogy with the Deuteron, a well known hadron molecule, with a binding energy of about $2.2 \mathrm{MeV}$. Their possible existence in the charmonium sector was already suggested very early on [44]. They were reinvented subsequently by Törnqvist [45,46], who proposed the existence of possible Deuteron-like states of two mesons, called Deusons. Bound together essentially by a pion exchange, they provide a plausible template for some of the exotica in the charmonium and bottomonium sectors. These ideas, borrowed essentially from nuclear bindings, have been further 
developed by a number of groups, in which the constituent heavy mesons rescatter to give rise to the final states in which the exotic hadrons are discovered. They are generically called hadronic molecules, reviewed comprehensively in a recent paper [15]. The last theoretical construct that we briefly discuss are QCD hybrids [47], consisting of a $Q \bar{Q} g$, where $g$ is a constituent gluon, typically having a mass of about $1 \mathrm{GeV}$. The $J^{P C}=1^{--} Y(4260)$ is the usual suspect here. Thus, some of the exotic hadrons may eventually turn out to be hybrids. In the theoretical part of this review, we discuss the salient features of these theoretical proposals, but elaborate the compact diquark model in somewhat more detail, as this represents the most radical and far reaching departure from the $q \bar{q}$ orthodoxy.

The main physics interest in this field is driven by the following questions: Are we at the threshold of a new frontier of QCD - with a vast and unexplored hadronic landscape - in which the compact diquarks and/or the hybrids play a central role? Or, are we witnessing the effects of a residual chromodynamic van der Waals force, surfacing in the form of hadronic molecules? Given enough data, the diquark models can be distinguished from the molecular interpretation, as in the former we expect complete $S U(3)_{F}$ multiplets, unlike the hadronic molecules. This becomes evident later in this review, as we work out the spectroscopy of the multiquark states in the diquark model. There are other differences in the production mechanisms and decays, such as the cross sections, $p_{T}$ distributions, and final state configurations. The cusp-based interpretation can be confirmed, or debunked, based on the phase motion of the amplitudes in question. This difference between a genuine resonance (a Breit-Wigner) and a cusp is well known and will be discussed later as well. In the coming years, the current experiments at the Large Hadron Collider (LHC), in particular LHCb, and BESIII, an experiment at the Beijing electron positron collider, will be joined by Belle II, at KEK in Japan, JLAB in the US, and PANDA at the planned $p \bar{p}$ facility at GSI, Darmstadt. The high luminosity LHC phase with an upgraded $\mathrm{LHCb}$ (and other detectors) will surely contribute greatly to this field. They will subject the current measurements and competing theoretical models to stringent tests. That this is a vibrant field is underscored by the observation of five new narrow $\Omega_{c}^{0}$ states in one go decaying to $\Xi_{c} K^{-}[48]$, which are in all likelihood quark-model states [49 52], and provide valuable information on the diquark binding in charm baryons. Their dynamics, interpreted in terms of a heavy (charm) quark and a light diquark (two strange quarks), can be worked out in the heavy-quark symmetry approach, and this may help elucidate the role of diquarks within tetraquarks and pentaquarks involving heavy quarks.3 ${ }^{3}$

This paper is organized as follows: In section 2, we briefly recall the salient features of the potential models in use in the quarkonium spectroscopy and review some of the ongoing lattice simulations in the excited and exotic charmonia sector. In section 3, we discuss in detail the experimental evidence for the pentaquark states $P_{c}^{+}(4380)$ and $P_{c}^{+}(4450)$ from LHCb in the decays $\Lambda_{b} \rightarrow p J / \psi K^{-}$and $\Lambda_{b} \rightarrow p J / \psi \pi^{-}$. We also discuss briefly the impending studies in the photoproduction process. In section 4 we summarize the experimental evidence for tetraquarks, starting with the $X(3872)$, and review various theoretical interpretations. Next in line is the state $X(3940)$, observed by Belle in double charmonium production $e^{+} e^{-} \rightarrow c \bar{c} c \bar{c}$. We then discuss the $Y$ states, in particular the $Y(4260)$, measured in the initial state radiation process $e^{+} e^{-} \rightarrow \gamma_{I S R} \gamma_{V}$, with $V \rightarrow Y(4260)$, observed by Belle in the invariant mass $m\left(J / \psi \pi^{+} \pi^{-}\right)$. The $Z_{c}$ states are taken up next, first observed by Belle in the decays $B^{0} \rightarrow \psi^{\prime} K^{ \pm} \pi^{ \pm}$, and review $Z_{c}(4430)$ and $Z_{c}(4200)$ in detail. The state $Z_{c}(3900)$, observed by BESIII in the decay $Y(4260) \rightarrow Z_{c}(3900)^{+} \pi^{-}$is discussed next (calling it the $Z_{c}$ state of type II). The $Z_{b}$ states in the bottomonium sector, $Z_{b}(10610)$ and $Z_{b}(10650)$, discovered by Belle in the decays $\Upsilon(5 S) \rightarrow Z_{b}(10610)^{+} \pi^{-}$and $\Upsilon(5 S) \rightarrow Z_{b}(10650)^{+} \pi^{-}$are the last of the $Z$ states we review. We close this section by discussing the resonant $J / \psi \phi$ states, discovered in a number of experiments (CDF, Belle, D0, CMS, LHCb). Theoretical models for tetraquarks are reviewed in section 5, which include the models discussed earlier, and the corresponding models for the pentaquarks are reviewed in section 6. We conclude with a brief summary and outlook in section 7.

\footnotetext{
${ }^{3}$ Luciano Maiani (private communication).
} 


\section{Theoretical techniques for quarkonia and exotic hadrons spectroscopy}

The current interest in the Quarkonium spectroscopy has shifted to excited states, in which the gluonic and light mesonic degrees of freedom are present in the Fock space description of a hadron. This includes the hybrid and tetraquark states, with the latter taking the form of two heavy-light mesons states, bound by pionic exchange (hadronic molecule), or genuine compact four-quark (tetraquark), states bound by gluons. Likewise, with the discovery of the two pentaquarks $P_{c}^{ \pm}(4380)$ and $P_{c}^{ \pm}(4450)$ by $\mathrm{LHCb}$, we have experimental evidence for the $c \bar{c} u u d$ states. The observed pentaquarks are found to have masses very close to a number of baryon-meson thresholds, as discussed quantitatively later. They could be the manifestation of these nearby thresholds in the scattering matrix, but they may also be genuine compact five-quark states. In this case, one anticipates also the bbuud states, and, in general, complete $S U(3)_{F}$ multiplets. So, in line with the experimental developments, the next frontier is to map out the spectrum of the $Q \bar{Q} g, Q \bar{Q} q \bar{q}$, and $Q \bar{Q} q q q$ states in $\mathrm{QCD}$, and figure out the underlying dynamics.

In this section, we review briefly the various theoretical techniques which have been used to calculate the spectroscopy of the excited quarkonia and exotic states. These include the potential models, which reflect the general features of QCD at short and long-distances and include spin-spin interactions among the quarks, and lattice QCD, in terms of the Born-Oppenheimer approximation, and simulations using current correlators with mesonic and diquark degrees of freedom. Also, very popular are the QCD sum rules, which have played an important role in understanding the properties of the light and heavy mesons, and which have also been applied for the studies of the exotic hadrons. However, as they have been covered extensively in a recent review [53], we will not discuss them here.

We start by briefly reviewing the Cornell non-relativistic potential [17, 18 and the Godfrey-Isgur model [54], which is its relativistic version, as both of them had a major impact on understanding the spectrum of the observed states and the transitions connecting the various quarkonium states.

\subsection{Quarkonium potentials}

The central Cornell potential is the standard color Coloumb plus a scalar linear form, and includes a hyperfine interaction. For the sake of definiteness we concentrate on the charmonium sector:

$$
V_{0}^{c \bar{c}}(r)=-\frac{4}{3} \frac{\alpha_{s}}{r}+b r+\frac{32 \pi \alpha_{s}}{9 m_{c}^{2}} \delta_{\sigma}(r) \vec{S}_{c} \cdot \vec{S}_{\bar{c}}
$$

where $\delta_{\sigma}(r)=(\sigma / \pi)^{3} e^{-\sigma^{2} r^{2}}$ is a Gaussian-smeared spin-spin contact interaction, a form taken from a later work [26]. There are four parameters, the effective strong coupling $\alpha_{s}$, string tension, $b$, charm quark mass, $m_{c}$, and the hyperfine coupling strength, $\sigma$. The remaining spin-independent terms of the potential are included in the leading order perturbation theory, and include the one-gluon-exchange spin-orbit and tensor interactions:

$$
V_{\alpha_{s}}^{c \bar{c}}(r)=\frac{1}{m_{c}^{2}}\left[\left(\frac{2 \alpha_{s}}{r^{3}}-\frac{b}{2 r}\right) \vec{L} \cdot \vec{S}+\frac{4 \alpha_{s}}{r^{3}} T\right] .
$$

There are just three independent spin-dependent operators, shown in Eqs. 1 and 2 . Of these, the tensor operator is defined in the configuration space as

$$
\vec{T} \equiv\left(\vec{S}_{c} \cdot \hat{r}\right)\left(\vec{S}_{\bar{c}} \cdot \hat{r}\right)-\frac{1}{3} \vec{S}_{c} \cdot \vec{S}_{\bar{c}}
$$

and the spin-orbit operators is $\vec{L} \cdot \vec{S}$, where $\vec{S}=\vec{S}_{c}+\vec{S}_{\bar{c}}$. They transform as $S=2$ and $S=1$, respectively. Hence, for states with $S=0$, their matrix elements vanish. The matrix elements also 
vanish for $L=0$ states. For $L>0$ and $S=1$, the total angular quantum number can assume the values $J=L-1, L$ and $L+1$.

The quarkonium potential-model studies were updated in 2005 [26] to incorporate the data from CLEO and the two $B$-factories. The parameters of the potential $V_{\mathrm{NR}}^{c \bar{c}}(r)=V_{0}^{c \bar{c}}(r)+V_{\alpha_{s}}^{c \bar{c}}(r)$ are fitted from the well-established $c \bar{c}$ states $1 S-4 S, 1 P$, and the two $D$-states $\psi(3770)$ and $\psi(4159)$. With only four parameters, $\left(\alpha_{s}, b, m_{c}, \sigma\right)$, the NR-cornell potential model is very predictive, and the entire normal charmonium spectrum was worked out. In particular, this allowed to predict the charmonium spectrum above the $D \bar{D}$ threshold, which includes $2 P, 3 P, 1 D, 2 D, 1 F, 2 F$, and $1 G$ states.

The Godfrey-Isgur model [54] assumes a relativistic dispersion relation for the quark kinetic energy, and an effective potential of the $c \bar{c}$ system, containing a Lorentz vector one-gluon exchange shortdistance and a scalar linear confining part for the long distance,

$$
\mathcal{H}_{G I}^{c \bar{c}}(p, r)=2 \sqrt{m_{c}^{2}+\mathbf{p}^{2}}+V_{\mathrm{eff}}^{c \bar{c}}(\mathbf{p}, r) .
$$

The effective potential $V_{\text {eff }}^{c \bar{c}}(\mathbf{p}, r)$ is derived from the on-shell $c \bar{c}$ amplitude, and given in Ref. [54]. In the non-relativistic limit, it reduces to the potential $V_{\mathrm{NR}}^{c \bar{c}}(r)$ given above, with $\alpha_{s}$ replaced by the running coupling constant. Hence, the fits yield different values for the parameters $b$ (string tension) and $m_{c}$. The spectra predicted by $V_{\mathrm{NR}}^{c \bar{c}}(r)$ and $\mathcal{H}_{G I}^{c \bar{c}}(p, r)$ are very similar for the $S$ - and $P$-wave states [26]. Both models predicted the charmonium spectroscopy quite accurately, including some of the states which lie above the corresponding open heavy meson thresholds.

However, not all the predicted charmonium states have been found, and several $2 P$ states in the charmonium sector, such as the $0^{++} \chi_{c 0}(2 P)$, the $1^{++} \chi_{c 1}(2 P)$, and the $1^{+-} h_{c}(2 P)$, are still missing or not identified unambiguously. The potential model-based estimates may receive mass shifts due to other effects, such as the couplings to open flavor channels [55], which are important if some of the states are found to have masses near thresholds. By construction, the potential models are not made to cover the excited charmonia (and bottomonia) states, and exotica, which have extra light degrees of freedoms in their Fock space, such as the hybrids $(Q \bar{Q} g)$ and the tetraquark states $Q \bar{Q} q \bar{q}$, where $q \bar{q}$ is a light quark $(q=u, d, s)$ pair. Their role is that they provide benchmarks to map out the normal quarkonia, and hence are very valuable in the search for the excited quarkonium states and exotica.

\section{$2.2 \quad$ Lattice simulations}

The spectroscopy of the mesons containing hidden and open-charm quarks calculated with the lattice techniques has made great strides lately. In particular, the calculations of the lowest-lying states well below the strong decay threshold have attained impressive precision with various systematic effects under control 22,56 58]. In lattice simulations, the mass of a single hadron is extracted from $m=E_{\vec{p}=0}$, extracted from the energies obtained from the $q \bar{q}$ or $q q q$ interpolating fields, extrapolated to the infinite volume, i.e. $L \rightarrow \infty$, with the lattice spacing $a \rightarrow 0$, and the quark mass $m_{q}^{\text {lattice }} \rightarrow m_{q}^{\text {phys }}$. For hadrons with heavy-light quarks $Q \bar{q}$ or with double heavy quark $Q \bar{Q}$, the lattice simulations use the Heavy Quark Effective Theory (HQET) or NRQCD, and expansions in either the heavy quark mass or the heavy quark velocity, and include four flavors $(u, d, s, c)$ of dynamical quarks. The current status of the spectroscopy for the heavy mesonic systems for the charm and bottom quarks is reviewed in the Particle Data Group (PDG) and compared with the lattice results (see Fig. 15.7 of the review by Amsler, DeGrand and Krusche in $[12]$ ). However, there are a number of states near the $D \bar{D}$ threshold, like $X(3872), Z_{c}^{ \pm}(3900)$ and $X(4140)$, whose quark composition is obscure.

A recent lattice work on the excited and exotic charmonium states is from the Hadron Spectrum Collaboration [59] where the excited spectrum is studied by varying the light quark mass. Using the $\eta_{c}$ mass to estimate the systematic error from tuning the charm quark mass yields an error of order $1 \%$ from this source. Adopting an anisotropic lattice formulation with the spatial lattice spacing $a_{s}=0.12$ fm and a temporal lattice spacing $a_{t}=3.5 a_{s}$, with $N_{f}=2+1$ flavor of dynamical quarks and a pion 


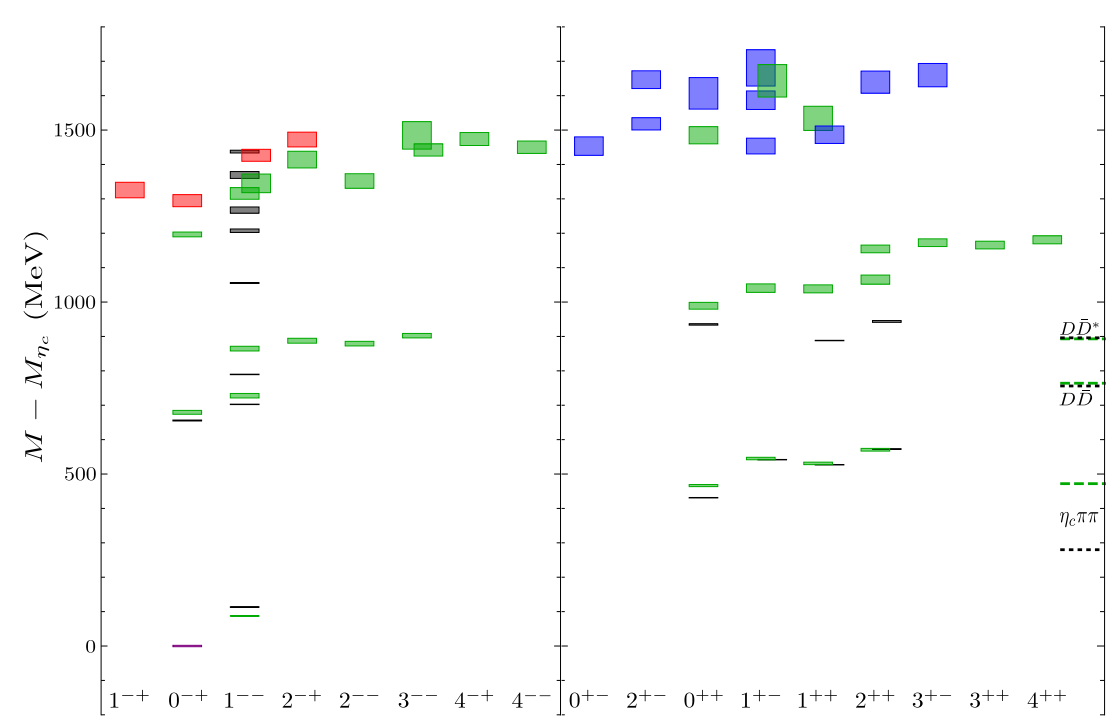

Figure 1: Charmonium spectrum up to $4.5 \mathrm{GeV}$, with the indicated $J^{P C}$ quantum numbers for the negative (positive) parity states shown in the left (right) panel. The calculated and experimental masses are shown in terms of $M-M_{\eta_{c}}$. The vertical size of the boxes represents the one-sigma statistical error. Red and blue boxes correspond to states identified as hybrid mesons, grouped into respectively, the lightest and first excited supermultiplet, and black boxes are experimental values from PDG. Location of some of the thresholds for strong decays are also indicated (from lattice QCD studies [59]).

mass $M_{\pi}=240 \mathrm{MeV}$, the result for the charmonium spectrum is presented in Fig. 1. The calculated and the experimental masses are compared with each other, with the $\eta_{c}$ mass subtracted to further reduce the uncertainty from the charm quark mass. Many of the states with non-exotic $J^{P C}$ quantum numbers follow the $n^{2 S+1} L_{J}$ pattern, predicted by the charmonium potential models, discussed earlier. All states up to $J=4$ are found. Fig. 1 also shows the states which do not fit the $n^{2 S+1} L_{J}$ pattern. Some of these have exotic $J^{P C}$ quantum numbers, $0^{+-}, 1^{-+}, 2^{+-}$. These and some extra non-exotic states are interpreted as hybrid mesons. The lightest hybrids appear about $1.2-1.3 \mathrm{GeV}$ above the lightest $S$-wave meson multiplets. This shows that a rich charmonium hybrid spectrum is waiting to be explored. The lattice mass estimates are in need of better systematic control to be quantitative, in particular, the current value of the pion mass used in these simulations is still quite high.

In the energy region near or above the strong decay thresholds, the masses of the bound-states and resonances are inferred from the infinite-volume scattering matrix of elastic or inelastic scattering. They represent one-channel and multiple-channel cases, respectively, with the inelastic case obviously technically more challenging. Various approaches with varying degrees of mathematical rigour are used in the simulation. The most widely used approach is based on Lüscher's formulation [60, 61] and its generalisation. Other approaches based on finite volume Hamiltonian Effective Field Theory (EFT) are also in use, in which the parameters are extracted by fitting the analytic expressions for the eigenvalues of the finite volume EFT using the lattice spectrum $E_{n}$ [62]. Likewise, the HALQCD approach 63], which is based on the lattice determination of the Nambu-Bethe-Salpeter wave functions for two hadrons as a function of their separation, has been employed to extract the potential $V(r)$ between the two hadrons. Their technical discussion will take us too far from the phenomenological focus of this review; they are reviewed in a number of papers $25,64,65$.

Several lattice results on the near-threshold bound states, scattering and resonances in the charm sector have been reported. Likewise, tetraquark spectroscopy has been investigated in a number of simulations, and there is at least one calculation on the lattice for pentaquarks [66]. A recent review on these lattice-based developments is Ref. [67]. In general, investigations of the excited charmonia and excited open charm mesons have systematic uncertainties, which are not quite accounted for. We 
discuss a sampling of these studies to illustrate the current state of this field.

$\mathbf{X}(\mathbf{3 8 7 2})$ : The $X(3872)$ lies very close to the $D^{0} \bar{D}^{* 0}$ threshold and it is essential to take into account the effect of this threshold on the lattice. This represents a shallow bound state from onechannel scattering. The first simulation of $D \bar{D}^{*}$ scattering was done in Ref. [68], where a pole in the scattering matrix was found just below the threshold in the $I=0, J^{P C}=1^{++}$channel. The pole was associated with the $X(3872)$, and was confirmed subsequently in a simulation by the Fermilablattice/MILC collaboration [69]. More recently, this topic was reinvestigated [70] by including diquarkantidiquark interpolating fields to determine which Fock components are essential for the $X(3872)$, suggesting that both the $c \bar{c}$ and the $D^{0} \bar{D}^{* 0}$ threshold are required for the $X(3872)$, rather than the diquark-antidiquark correlator. If confirmed by theoretically improved calculations with controlled systematic errors, this would imply that the $X(3872)$, the trailblazer of the exotica, is indeed a $c \bar{c}$ state whose mass is modified by the threshold.

$\mathbf{Z}_{\mathbf{c}}^{+}\left(\mathbf{3 9 0 0 )}\right.$ : Lattice simulation of the $Z_{c}^{+}(3900)$, which has the quark flavor content $c \bar{c} u \bar{d}$ and has the quantum numbers $I^{G}\left(J^{P C}\right)=1^{+}\left(1^{+-}\right)$, is reported in Ref. 71]. This is studied by the method of coupled-channel scattering involving the final states $D \bar{D}^{*}, \pi J / \bar{\psi}$ and $\rho \eta_{c}$, for which the HALQCD approach [63] is used. It involves a calculation of the potential for the $3 \times 3$ scattering matrix. First the potential $V_{\pi J / \psi \rightarrow \pi J / \psi}(r)$ related to the Nambu-Bethe-Salpeter equation is determined between the $J / \psi$ and $\pi$ as a function of their separation $r$. The potential for the other two channels $D \bar{D}^{*}$ and $\rho \eta_{c}$ and the off-diagonal elements involving these channels are likewise calculated, and used to determine the three-body decay $Y(4260) \rightarrow J / \psi \pi \pi$ and $Y(4260) \rightarrow D \bar{D}^{*}$ in a phenomenological way. Indeed, a peak around the $Z_{c}^{+}(3900)$ is found. If the coupling between the $D \bar{D}^{*}$ and $\pi J / \psi$ is switched off, the peak disappears, which reflects that the potential for the off-diagonal element $D \bar{D}^{*}$ and $\pi J / \psi$ is larger than the other potentials. Hence, this study suggests that also the $Z_{c}^{+}(3900)$ is possibly a rescattering effect. However, the simulation in Ref. [71] is not done using the rigorous Lüscher's formulation, and hence the conclusions are generally not accepted by the lattice community [67].

Pentaquarks: Attempts to simulate the LHCb-type pentaquarks on the lattice are also under way. The NPLQCD collaboration [66] has presented first evidence for a $\eta_{c} N$ bound state approximately 20 $\mathrm{MeV}$ below the $\eta_{c} N$ threshold - again a case of a shallow one-channel scattering, similar to the $X(3872)$, but now in the baryonic sector. The simulation is done at an $S U(3)_{F}$-symmetric point corresponding to $m_{\pi}=800 \mathrm{MeV}$. This is far afield from the physical mass of the pion, and it is not clear if the evidence for the bound state will persist for more realistic pion mass. The observed pentaquarks $P_{c}^{+}(4380)$ and $P_{c}^{*}(4450)$ are, on the other hand, about $400 \mathrm{MeV}$ above the $J / \psi p$ threshold. The lattice simulation of these pentaquarks is much more challenging as it is a multi-channel problem, with several open thresholds nearby. It would be exciting if experimentally a bound state is found in the $\eta_{c} N$ channel hinted by the NLPQCD simulation.

\subsection{Born-Oppenheimer tetraquarks}

The Born-Oppenheimer (B-O)approximation, which was introduced in 1927 to study the binding of atoms into molecules [72, makes use of the large ratio of the masses of the atomic nucleus and the electron. The nuclei can be approximated by static sources for the electric field, and the electrons respond almost instantaeneously to the motion of the nuclei. The implicit adiabatic approximation reduces the rather intricate dynamics to the tractable problem of calculating the B-O potentials, which are defined by the Coulomb energy of the nuclei and the energy of the electrons. The QCD analog of this is that the nucleus is replaced by a heavy $Q \bar{Q}$ (or $Q Q$ ), pair, and the electron cloud is replaced by the light degrees of freedom, a gluon for a $Q \bar{Q} g$ hybrid, or a light $q \bar{q}$ (or $\bar{q} \bar{q}$ ) pair for a tetraquark. If the masses of the two heavy quarks are much larger than the QCD scale, $\Lambda_{\mathrm{QCD}}$, which is the case for the 
bottom and charm quarks, then the dynamics can be described by a quantum mechanical Hamiltonian with an appropriate QCD potential, which can be studied on the lattice.

The B-O approximation for $Q \bar{Q}$ mesons in QCD was studied by Juge, Kuti, and Morningstar [73] who investigated the hybrid $b \bar{b} g$ molecules, a bound system of $b \bar{b}$ and excited gluon field, and also carried out detailed studies in the lattice quenched approximation. The consistencey of the result from the two approaches made a compelling case for the heavy hybrid states. The interest in the B-O approximation was revived in Ref. $[74$ to cover also the flavor-nonsinglet $Q \bar{Q}$ mesons, in particular, the $X Y Z$ tetraquarks. The B-O potential involves a single-channel approximation that simplifies the Schrödinger equation, which can be solved for just one radial wavefunction. In that case, the B-O approximation offers a reliable template for a coherent description of these hadrons in QCD. On the other hand, if the masses of the $X Y Z$ tetraquarks are close to the thresholds for a pair of heavy mesons, which is often the case, then one has to account for the coupling to the meson pair scattering, and the Schrödinger equation becomes a multi-channel problem. This requires detailed lattice calculations of the B-O potentials to estimate the effects of the couplings between the channels, which has still to be carried out for the $X Y Z$ hadrons.

\subsection{Doubly heavy tetraquarks on the lattice}

The case of two heavy antiquarks $(\bar{Q} \bar{Q})$ and a light $q q$ pair, bound in a hadron, has also received a lot of theoretical interest, though so far there is no trace of hadrons, such as $u d \bar{b} \bar{b}$ or $u s \bar{b} \bar{b}$ experimentally. Such exotics are difficult to produce in high energy experiments, with presumably LHC the only collider where they may show up albeit with a very small cross section. If found, they would be truly exotic. As we focus mainly on the observed pentaquarks and tetraquarks in the rest of this review, we briefly discuss two recent theoretical estimates of the doubly heavy tetraquarks masses and decay widths [75, 76].

In Ref. [75, the potential of two static antiquarks $\bar{Q} \bar{Q}$ in the presence of two light quarks $q q$ is parametrized by a screened Coulomb potential

$$
V(r)=-\frac{\alpha}{r} e^{-r^{2} / d^{2}}
$$

which is inspired by one-gluon exchange at small $\bar{Q} \bar{Q}$ separation $r$ and a screening of the Coulomb potential due to the formation of two $\bar{B}$-mesons at large $r$. The parameters $\alpha$ and $d$ depend on the isospin $I$ and the total angular momentum $j$ of the light $q q$ pair. They are determined on the lattice by fitting the ground state potentials in the attractive channels, and it is found that the $(I=0, j=0)$ potential is more attractive than $(I=1, j=1)[77]$, yielding $\alpha=0.34 \pm 0.03$ and $d=\left(0.45_{-0.10}^{+0.12}\right) \mathrm{fm}$. With this potential the Schrödinger equation is solved: $\left(H_{0}+V(r)-E\right) X=-V(r) \psi_{0}$, where $X$ is defined by the wavefunction splitting $\psi=\psi_{0}+X$, with $\psi_{0}$ the incident and $X$ the emergent wave. From the asymptotic behavior of $X$, the phase shift, $\delta_{\ell}$ is determined as a function of the energy $E$, which is continued to the complex E-plane. Analyticity is used to determine the pole position of the resonance and its width, yielding $m=10576 \pm 4 \mathrm{MeV}$ and $\Gamma=112_{-103}^{+90} \mathrm{MeV}$ for the $u d \bar{b} \bar{b}$ charged exotic having the quantum numbers $I\left(J^{P}\right)=0\left(1^{-}\right)[75$.

The possibility of a doubly heavy tetraquark $q q^{\prime} \bar{b} \bar{b}$ bound states has also recently been studied on the lattice, using NRQCD to simulate the bottom quarks [76]. A two-point lattice QCD correlation function is defined in the Euclidean time

$$
C_{\mathcal{O}_{1} \mathcal{O}_{2}}(p, t)=\sum_{n}\left\langle 0\left|\mathcal{O}_{1}\right| n\right\rangle\left\langle n\left|\mathcal{O}_{2}\right| 0\right\rangle e^{-E_{n}(p) t}
$$

where the operators $\left(\mathcal{O}_{i}\right)$ have the appropriate quantum numbers. Thus, for the $B(5279)$ meson, with $I\left(J^{P}\right)=\frac{1}{2}\left(0^{-}\right)$, and $B^{*}(5325)$, with $I\left(J^{P}\right)=\frac{1}{2}\left(1^{-}\right)$, the interpolating fields are the $q \bar{q}$ bilinears $P \sim \bar{b} \gamma_{5} q$ 
and $V \sim \bar{b} \gamma_{i} q$, respectively. They are used to define a two-meson operator $M(x)$ having the quantum number $J^{P}=1^{+}$:

$$
M(x)=\bar{b}_{a}^{\alpha}(x) \gamma_{5}^{\alpha \beta} u_{a}^{\beta}(x) \bar{b}_{b}^{\kappa}(x) \gamma_{i}^{\kappa \rho} d_{b}^{\rho}(x)-\bar{b}_{a}^{\alpha}(x) \gamma_{5}^{\alpha \beta} d_{a}^{\beta}(x) \bar{b}_{b}^{\kappa}(x) \gamma_{i}^{\kappa \rho} u_{b}^{\rho}(x),
$$

and an analogous operator with the $B_{s} B^{*}$ structure. Here, $a, b$ are color indices, and $\alpha, \beta, \ldots$ are Dirac indices .

The second operator $D(x)$ has the diquark-antidiquark structure with $\bar{b} \bar{b}$ a color triplet $3_{c}$, spin 1 , and the light diquark having the flavor, spin, color quantum numbers $\left(\overline{3}_{F}, 0, \overline{3}_{c}\right)$,

$$
D(x)=\left(\left[u_{a}^{\alpha}(x)\right]^{T}\left(C \gamma_{5}\right)^{\alpha \beta} q_{b}^{\beta}(x)\right)\left(\bar{b}_{a}^{\kappa}(x)\left(C \gamma_{i}\right)^{\kappa \rho}\left[\bar{b}_{b}^{\rho}(x)\right]^{T}\right),
$$

where $q=d, s$. This yields a $J^{P}=1^{+}$state. With this the binding correlator

$$
G_{\mathcal{O}_{1} \mathcal{O}_{2}}(p, t)=\frac{C_{\mathcal{O}_{1} \mathcal{O}_{2}}(p, t)}{C_{P P(t)} C_{V V(t)}}
$$

is studied. For a channel with a tetraquark ground state with (negative) binding energy $\Delta E$, with respect to the two-meson $P V$ threshold, this correlator grows as $e^{-\Delta E t}$. With this, the $(2 \times 2)$ matrix with the matrix elements $G_{D D}(t), G_{D M}(t), G_{M D}(t), G_{M M}(t)$ are studied and the two eigenvalues extracted, yielding the states $u d \bar{b} \bar{b}$ and $\ell s \bar{b} \bar{b}$, with $\ell=u, d$. They lie, respectively, 189(10) and 98(7) MeV below the corresponding thresholds, with $J^{P}=1^{+}$. These double $b$-quark tetraquarks are stable both with respect to the strong and electromagnetic decays, and will decay weakly. The discovery modes are listed as $B^{+} \bar{D}^{0}$ and $J / \psi B^{+} K^{0}$ for the first, and $J / \psi B_{s} K^{+}$and $J / \psi B^{+} \phi$ for the second.

In concluding this section on quarkonium potentials and lattice simulations, we note that the two approaches agree remarkably with each other for the case of single hadrons well below the strong decay thresholds. However, for the cases where the elastic or the inelastic scatterings are involved, reliable results from the lattice are not yet quantitative. This problem becomes much more complicated if several channels lie near each other. For tetraquarks with doubly heavy quarks $u d \bar{b} \bar{b}$ and $\ell s \bar{b} \bar{b}$, with $\ell=u, d$, lattice results have been obtained, and remain to be tested against experiments. The B-O approximation can yield reliable results for the single-channel case, but requires further study for the states where scattering thresholds have to be taken into account.

\section{Experimental evidence for pentaquarks}

\subsection{History}

The prospect of multi-quark hadrons was raised first by Jaffe in 1976 [34], and expanded upon by Strottman [78 to include baryons composed of four-quarks plus one antiquark. The name pentaquark was coined by Lipkin who predicted states with charmed quarks $[79]$. The concept of stable pentaquarks was mentioned around the same time by Gignoux et al. [80].

Pentaquark states composed only of $u, d$ and $s$ quarks have been previously reported to great fanfare, circa 2004. However, they have been debunked by updated analyses. The Hicks article [81] gives a good review of previous claims and their refutations. A brief summary will be given here. The story starts with observations of a bump in the invariant kaon nucleon mass spectra was observed at about $1.54 \mathrm{GeV}$ in various reactions listed in Table 1. The widths were rather narrow, around $10 \mathrm{MeV}$, quite surprising for pentaquarks that could easily decay, and thus should have a larger widths.

One notes these were all nuclear physics experiments whose significances did not exceed 5 standard deviation and whose statistics was rather low, so full amplitude analyses were not undertaken. These results were followed by the announcement of $\Xi^{--}$pentaquark state by NA49 [86. This state, however, 
Table 1: The first four experiments with positive evidence for the $\Theta^{+}$.

\begin{tabular}{|c|c|c|c|}
\hline Experiment & Reaction & Mass (GeV) & Significance \\
\hline LEPS 82$]$ & $\gamma C \rightarrow K^{+} K^{-} X$ & $1.54 \pm 0.01$ & $4.6 \sigma$ \\
\hline DIANA 83 & $K^{+} X e \rightarrow K_{\mathrm{S}}^{0} p X$ & $1.539 \pm 0.002$ & $4.4 \sigma$ \\
\hline CLAS 84 & $\gamma d \rightarrow K^{+} K^{-} p n$ & $1.542 \pm 0.005$ & $(5.2 \pm 0.6) \sigma$ \\
\hline SAPHIR 85 & $\gamma p \rightarrow K_{\mathrm{S}}^{0} K^{+} n$ & $1.540 \pm 0.004$ & $4.8 \sigma$ \\
\hline
\end{tabular}

was not seen by the HERA-B collaboration, that had a larger data sample [87]. Soon thereafter the pentaquark fervor was stoked by a paper from the $\mathrm{H} 1$ collaboration that showed evidence for a pentaquark containing $u d u d \bar{c}$ quarks, a charmed pentaquark [88], seen in the $D^{*-} p$ decay mode that was quickly contradicted by the Zeus experiment [89].

All of the positive results were controversial. There was an attempt to explain the data from kinematical reflections [90]. There then followed a period with several "confirmations" of the $\Theta^{+}$but also many other experiments that did not see it. CLAS, for example, with a 20 times larger data sample than their original result, showed that the state was not present. Very high statistics experiments such as BaBar did not see any of these states. (References to these articles can be found in [81]). Currently there is no obvious explanation of the positive results, although this may be an additional example of "pathological science" 91].

\subsection{The LHCb observation of pentaquark charmonium states}

\subsubsection{The $\Lambda_{b}^{0} \rightarrow J / \psi K^{-} p$ decay}

The principle aim of the LHCb experiment is to find physics beyond the Standard Model using rare and $C P$-violating decays of $b$-flavored hadrons [92]. During one of these studies involving $B \rightarrow J / \psi K^{+} K^{-}$ decays a question of a possible background was raised from an as yet to be observed decay $\Lambda_{b}^{0} \rightarrow$ $J / \psi K^{-} p$, where the $p$ was misidentified as a $K^{+}$meson. Note, at the LHC $\Lambda_{b}^{0}$ baryons are prolifically produced. In the acceptance of the $\mathrm{LHCb}$ experiment $\sim 20 \%$ of all $b$-flavored hadrons are $\Lambda_{b}^{0}$ 's $[93]$. It was quickly realized that this decay mode offered an excellent way to measure the $\Lambda_{b}^{0}$ lifetime as it had a large event yield, and had four charged tracks that were used to define the coordinates of the $\Lambda_{b}^{0}$ decay point. The reconstructed $J / \psi K^{-} p$ invariant mass distribution is shown in Fig. 2(left) showing about 26,000 signal candidates along with 1,400 background events within $\pm 15 \mathrm{MeV}$ of the mass peak.
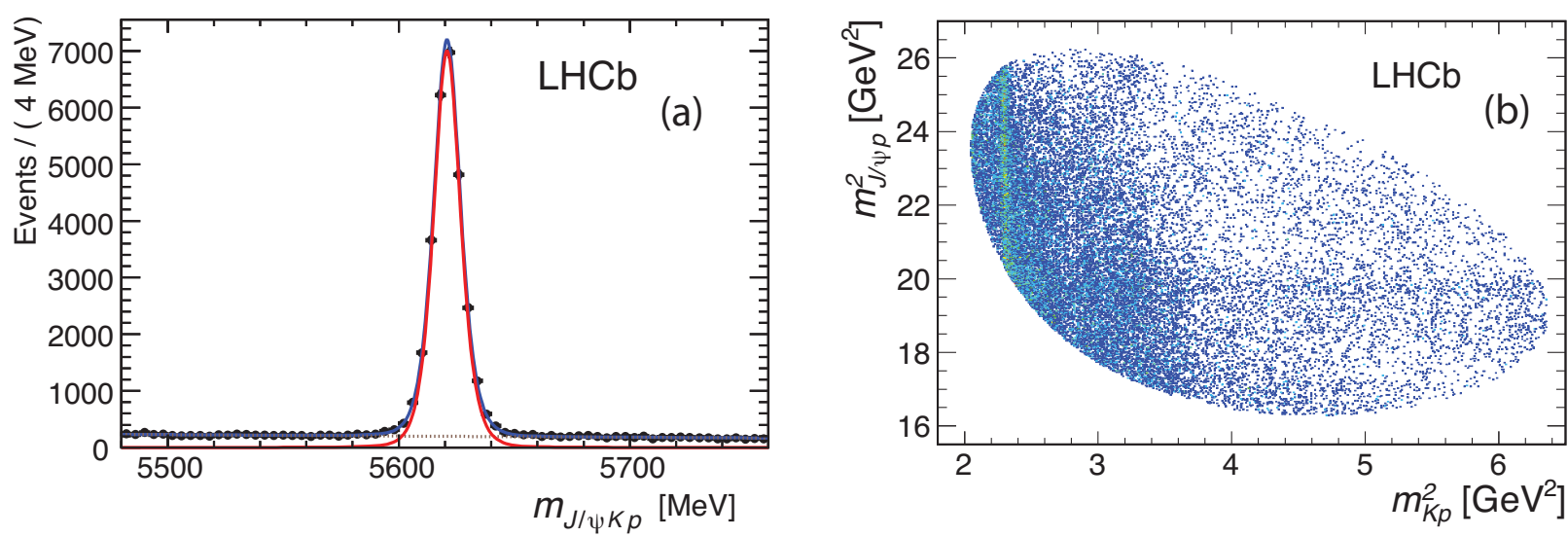

Figure 2: (a) Invariant mass spectrum of $J / \psi K^{-} p$ combinations, with the total fit, signal and background components shown as solid (blue), solid (red) and dashed lines, respectively. (b) Invariant mass squared of $K^{-} p$ versus $J / \psi p$ for candidates within $\pm 15 \mathrm{MeV}$ of the $\Lambda_{b}^{0}$ mass (from [1]). 

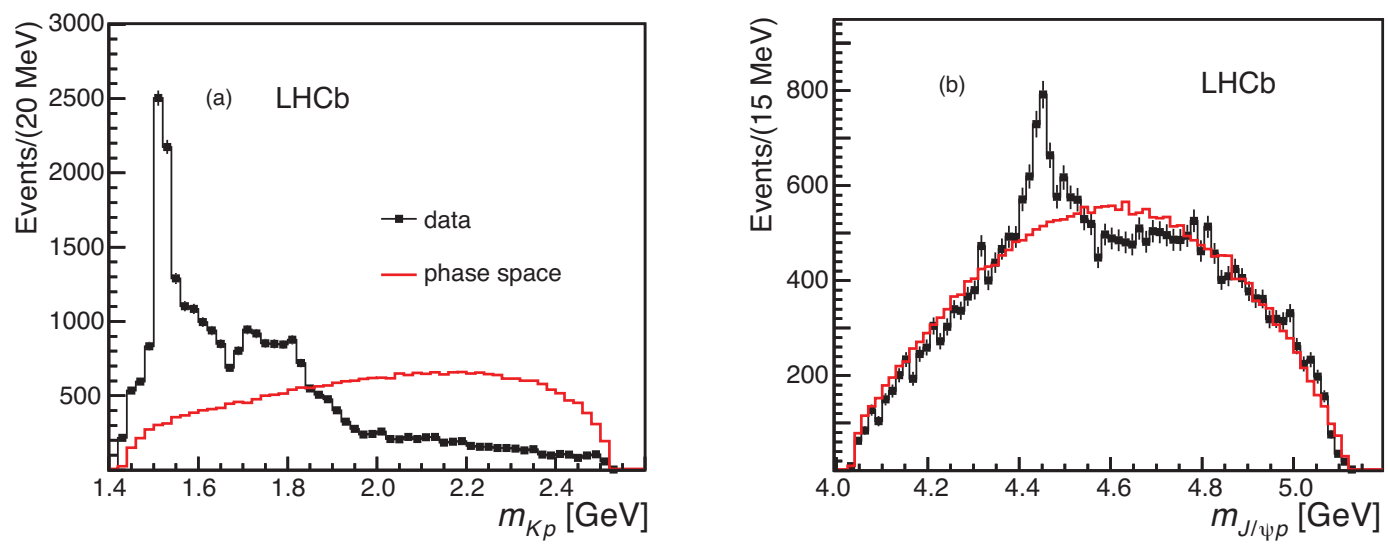

Figure 3: Invariant mass of (a) $K^{-} p$ and (b) $J / \psi p$ combinations from $\Lambda_{b}^{0} \rightarrow J / \psi K^{-} p$ decays. The solid (red) curve is the expectation from phase space. The background has been subtracted (from [11]).

A consequence of this discovery was that the $\Lambda_{b}^{0}$ lifetime was measured precisely, which settled an important issue as many previous measurements showed a large difference with other $b$-flavored hadron species that was not predicted and not confirmed by LHCb [94]. However, examination of the decay products showed an anomalous feature [11]. The Dalitz like plot [95] shown in Fig. 2(right) using the $K^{-} p$ and $J / \psi p$ invariant masses-squared as independent variables ${ }^{4}$ There are vertical bands corresponding to $\Lambda^{*} \rightarrow K^{-} p$ resonant structures, and an unexpected horizontal band near $19.5 \mathrm{GeV}^{2}$.

The Dalitz plot projections are shown in Fig. 3. Indeed there are significant structures in the $K^{-} p$ mass spectrum that differ from phase space expectations, and there is also a peak in the $J / \psi p$ mass spectrum. The leading order Feynman diagrams for $\Lambda_{b}^{0} \rightarrow J / \psi \Lambda^{*}$, and for $\Lambda_{b}^{0} \rightarrow K^{-} P_{c}^{+}$, where $P_{c}^{+}$is a possible state that decays into $J / \psi p$, are shown in Fig. 4.

This decay can proceed by the diagram shown in Fig. 4(a), and is expected to be dominated by $\Lambda^{*} \rightarrow K^{-} p$ resonances, as are evident in the data shown in Fig. 3(a). It could also have exotic contributions, as indicated by the diagram in Fig. 4(b), that could result in resonant structures in the $J / \psi p$ mass spectrum shown in Fig. 3(b).

In order to establish the resonant content of this $\Lambda_{b}^{0}$ decay it is necessary to consider both $\Lambda_{b}^{0} \rightarrow J / \psi \Lambda^{*}$ and $\Lambda_{b}^{0} \rightarrow K^{-} P_{c}^{+}$decay sequences simultaneously. LHCb, however, had to consider first consider the possibility that interferences among only the $\Lambda^{*}$ decay chain caused a peak in the $J / \psi p$ mass spectrum. So at first only the amplitudes described by the diagram in Fig. 4(a) were considered.

Even with this restriction there are many final states. The decays $\Lambda^{*} \rightarrow K^{-} p$ and $\Sigma^{*} \rightarrow K^{-} p$ are both, in principle, possible. Note however, that the isospin of the initial $\Lambda_{b}^{0}$ baryon is zero, as is the isospin of the $J / \psi$ meson, so if the resonant state were to be a isospin one $\Sigma^{*}$ rather than a $\Lambda^{*}$ we would have a change of isospin of one unit in the weak decay, which even though possible is thought to be highly suppressed, similar to the situation in the decay of the kaon into two pions [96] where the

\footnotetext{
${ }^{4}$ The Dalitz plot was conceived for decays into three scalar mesons, where the phase space is uniform over the plot area, so that the effects of the matrix element governing the decay are directly visible.
}
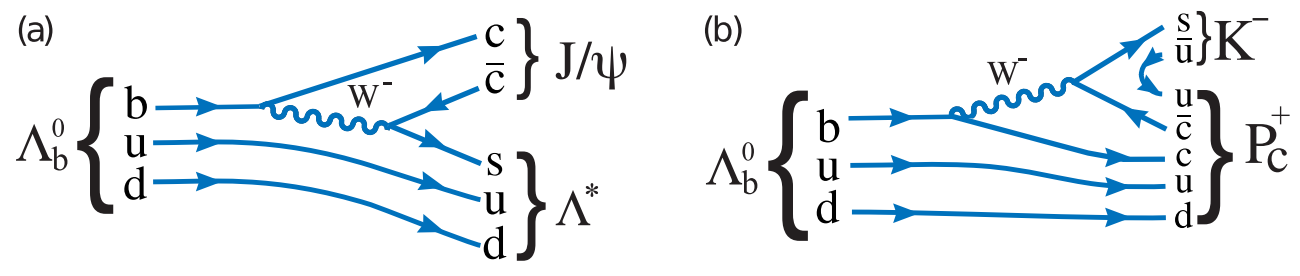

Figure 4: Feynman diagrams for (a) $\Lambda_{b}^{0} \rightarrow J / \psi \Lambda^{*}$ and (b) $\Lambda_{b}^{0} \rightarrow P_{c}^{+} K^{-}$decay (from [11]). 
isospin changing $3 / 2$ amplitude is highly suppressed with respect to the $1 / 2$ amplitude. The $\Lambda^{*}$ states are listed as the "Extended" model in Table 2, Along with each state the number of possible decay amplitudes with different orbital angular momentum $L$, and spin $S$ quantum numbers are also given. Of course it is not apriori known which of these states and amplitudes is present, or even if there are additional states that have yet to be discovered which also contribute.

Table 2: The $\Lambda^{*}$ resonances used in the different fits. Parameters are taken from the PDG [12]. We take $5 / 2^{-}$for the $J^{P}$ of the $4(2585)$. The number of $L S$ couplings is also listed for both the "reduced" and "extended" models. To fix overall phase and magnitude conventions, which otherwise are arbitrary, we set $B_{0, \frac{1}{2}}=(1,0)$ for $\Lambda(1520)$. A zero entry means the state is excluded from the fit.

\begin{tabular}{lccccc}
\hline State & $J^{P}$ & $M_{0}(\mathrm{MeV})$ & $\Gamma_{0}(\mathrm{MeV})$ & \# Reduced & \# Extended \\
\hline$\Lambda(1405)$ & $1 / 2^{-}$ & $1405.1_{-1.0}^{+1.3}$ & $50.5 \pm 2.0$ & 3 & 4 \\
$\Lambda(1520)$ & $3 / 2^{-}$ & $1519.5 \pm 1.0$ & $15.6 \pm 1.0$ & 5 & 6 \\
$\Lambda(1600)$ & $1 / 2^{+}$ & 1600 & 150 & 3 & 4 \\
$\Lambda(1670)$ & $1 / 2^{-}$ & 1670 & 35 & 3 & 4 \\
$\Lambda(1690)$ & $3 / 2^{-}$ & 1690 & 60 & 5 & 6 \\
$\Lambda(1800)$ & $1 / 2^{-}$ & 1800 & 300 & 4 & 4 \\
$\Lambda(1810)$ & $1 / 2^{+}$ & 1810 & 150 & 3 & 4 \\
$\Lambda(1820)$ & $5 / 2^{+}$ & 1820 & 80 & 1 & 6 \\
$\Lambda(1830)$ & $5 / 2^{-}$ & 1830 & 95 & 1 & 6 \\
$\Lambda(1890)$ & $3 / 2^{+}$ & 1890 & 100 & 3 & 6 \\
$\Lambda(2100)$ & $7 / 2^{-}$ & 2100 & 200 & 1 & 6 \\
$\Lambda(2110)$ & $5 / 2^{+}$ & 2110 & 200 & 1 & 6 \\
$\Lambda(2350)$ & $9 / 2^{+}$ & 2350 & 150 & 0 & 6 \\
$\Lambda(2585)$ & $?$ & $\approx 2585$ & 200 & 0 & 6 \\
\hline
\end{tabular}

\subsubsection{The $\Lambda_{b}^{0} \rightarrow J / \psi \Lambda^{*}$ decay amplitude}

To identify the $\Lambda^{*}$ states present one could try to fit the $m_{K p}$ mass distribution with some or all of the resonances listed in Table 2. This procedure, however, would only use part of the information available in the data. The correlations among the decay angular distributions of the final state particles contains information on the spin-parities of intermediate $\Lambda^{*}$ resonances. In fact, the decay of the $\Lambda_{b}^{0}$ into $J / \psi \Lambda$ with $J / \psi \rightarrow \mu^{+} \mu^{-}$and $\Lambda^{*} \rightarrow K^{-} p$ is fully characterized by $K^{-} p$ invariant mass and the five angular variables shown in Fig. 5. The $\Lambda_{b}^{0}$ decay plane is defined by the cross product of the $\psi$ and $\Lambda^{*}$ three vectors, while the $\psi$ decay plane defined by the cross product of the $\mu^{+}$and $\mu^{-}$three vectors, and the $\Lambda^{*}$ decay plane by the cross product of the $K^{-}$and $p$ three vectors. The angle between the $\Lambda_{b}^{0}$ rest frame and the $\psi$ rest frame is denoted as $\phi_{\mu}$, while the angle of the $\mu^{-}$in the $\psi$ rest frame with respect to the $\psi$ direction is called $\theta_{\psi}$. Similarly, the angle between the $\Lambda_{b}^{0}$ rest frame and the $\Lambda^{*}$ rest frame is denoted as $\phi_{K}$, while the angle of the $K^{-}$in the $\Lambda^{*}$ rest frame with respect to the $\Lambda^{*}$ direction is called $\theta_{\Lambda^{*}}$.

In order to fit the data to determine its resonant content, it is necessary to express the total decay amplitude in terms of these variables and the allowed individual decay amplitudes for each allowed $\Lambda^{*}$ resonance decays listed in Table 2. The mathematical expression for this amplitude is derived in the supplementary material of Ref. [11]. Besides finding the correct angular expressions, the amplitude uses Breit-Wigner functions for the $m_{K p}$ distributions except for the $\Lambda(1405)$ where a Flatté parameterization [97] is taken. These functions are labeled as $R_{A}\left(m_{K p}\right)$ in the expression below and $R_{\Lambda^{*} n}\left(m_{K p}\right)$ in Eq. [12 


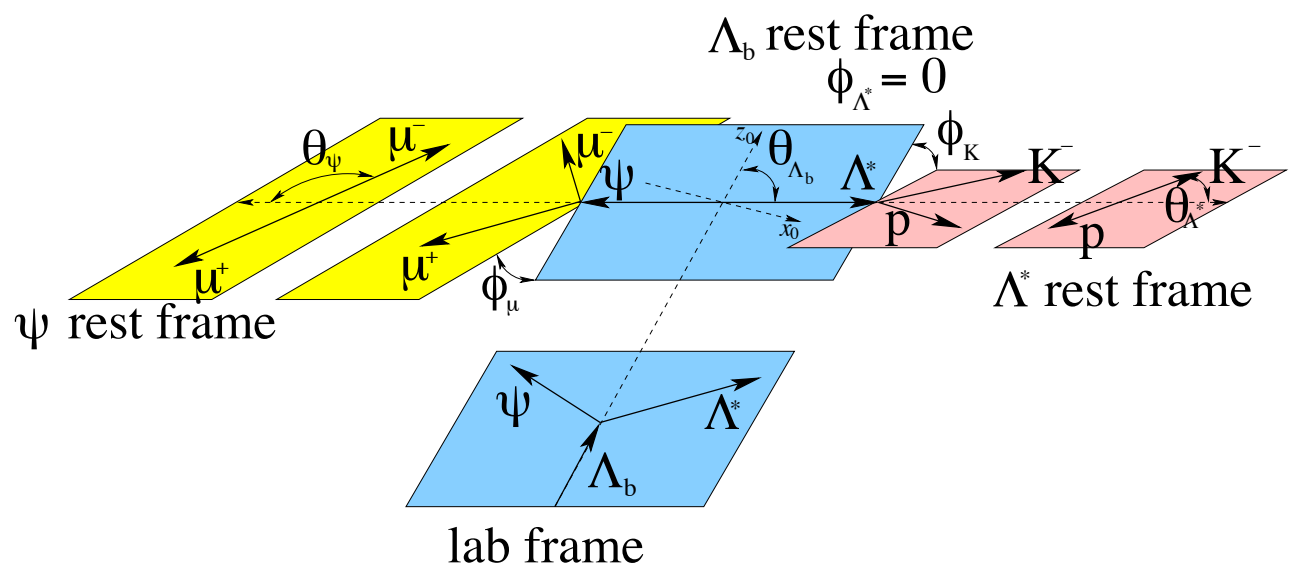

Figure 5: Definition of the decay angles in the $\Lambda^{*}$ decay chain (from [1]|).

It is customary to use the helicity formalism 98 100] where each sub-decay $A \rightarrow B C$ contributes a term to the amplitude:

$$
\begin{aligned}
\mathcal{H}_{\lambda_{B}, \lambda_{C}}^{A \rightarrow B C} D_{\lambda_{A}, \lambda_{B}-\lambda_{C}}^{J_{A}}\left(\phi_{B}, \theta_{A}, 0\right)^{*} R_{A}\left(m_{B C}\right)= & \mathcal{H}_{\lambda_{B}, \lambda_{C}}^{A \rightarrow B C} e^{i \lambda_{A} \phi_{B}} d_{\lambda_{A}, \lambda_{B}-\lambda_{C}}^{J_{A}}\left(\theta_{A}\right) \\
& \times R_{A}\left(m_{B C}\right) .
\end{aligned}
$$

The $\lambda$ 's are the helicity quantum numbers given by the projection of the particles spin in the direction of its momentum vector, and $\mathcal{H}_{\lambda_{B}, \lambda_{C}}^{A \rightarrow B C}$ are complex helicity-coupling amplitudes describing the decay dynamics. Here $\theta_{A}$ and $\phi_{B}$ are the polar and azimuthal angles of $B$ in the rest frame of $A\left(\theta_{A}\right.$ is often called the "helicity angle" of $A$ ). $D$ is the Wigner matrix whose three arguments are Euler angles describing the rotation of the initial coordinate system with the $z$-axis along the helicity axis of $A$ to the coordinate system with the $z$-axis along the helicity axis of $B[12]$. The convention is chosen which sets the third Euler angle to zero. In Eq. 10$), d_{\lambda_{A}, \lambda_{B}-\lambda_{C}}^{J_{A}}\left(\theta_{A}\right)$ is the small- $d$ Wigner matrix.

Using Clebsch-Gordan coefficients, the helicity couplings can be written in terms of $L S$ couplings $\left(B_{L, S}\right)$, where $L$ is the orbital angular momentum in the decay, and $S$ is the total spin of $A$ plus $B$ :

$$
\begin{aligned}
\mathcal{H}_{\lambda_{B}, \lambda_{C}}^{A \rightarrow B C}=\sum_{L} \sum_{S} \sqrt{\frac{2 L+1}{2 J_{A}+1}} B_{L, S} & \left(\begin{array}{cc|c}
J_{B} & J_{C} & S \\
\lambda_{B} & -\lambda_{C} & \lambda_{B}-\lambda_{C}
\end{array}\right) \times \\
& \left(\begin{array}{cc|c}
L & S & J_{A} \\
0 & \lambda_{B}-\lambda_{C} & \lambda_{B}-\lambda_{C}
\end{array}\right),
\end{aligned}
$$

where the expressions in parentheses are the standard Wigner 3j-symbols. For strong decays, possible $L$ values are constrained by the conservation of parity: $P_{A}=P_{B} P_{C}(-1)^{L}$.

The matrix element for the $\Lambda_{b}^{0} \rightarrow J / \psi \Lambda^{*}$ decay sequence is

$$
\begin{aligned}
\mathcal{M}_{\lambda_{\Lambda_{b}^{0}}, \lambda_{p}, \Delta \lambda_{\mu}}^{\Lambda^{*}} \equiv & \sum_{n} \sum_{\lambda_{\Lambda^{*}}} \sum_{\lambda_{\psi}} \mathcal{H}_{\lambda_{\Lambda^{*}, \lambda_{\psi}}^{\Lambda^{0} \rightarrow \Lambda^{*} n \psi}} D_{\lambda_{\Lambda_{b}^{0}}}^{\frac{1}{2}} \lambda_{\Lambda^{*}-\lambda_{\psi}}\left(0, \theta_{\Lambda_{b}^{0}}, 0\right)^{*} \mathcal{H}_{\lambda_{p}, 0}^{\Lambda^{*} n \rightarrow K p} \\
& D_{\lambda_{\Lambda^{*}}, \lambda_{p}}^{J_{\Lambda^{*} n}}\left(\phi_{K}, \theta_{\Lambda^{*}}, 0\right)^{*} R_{\Lambda^{*} n}\left(m_{K p}\right) D_{\lambda_{\psi}, \Delta \lambda_{\mu}}^{1}\left(\phi_{\mu}, \theta_{\psi}, 0\right)^{*}
\end{aligned}
$$

where the $x$-axis, in the coordinates describing the $\Lambda_{b}^{0}$ decay, is chosen to fix $\phi_{\Lambda^{*}}=0$. The sum over $n$ allows several different $\Lambda^{*} n$ resonances to contribute to the amplitude. Since the $J / \psi$ decay is electromagnetic, the values of $\Delta \lambda_{\mu} \equiv \lambda_{\mu^{+}}-\lambda_{\mu^{-}}$are restricted to \pm 1 .

\subsubsection{Fits of the reaction $\Lambda_{b}^{0} \rightarrow J / \psi \Lambda^{*}$ to the data}

The next step is to fit the square of the matrix element given in Eq. 13 to the background subtracted and efficiency corrected data. One method of subtracting the background uses the sidebands on either 
side of the $\Lambda_{b}^{0}$ mass peak to provide a sample of events. The efficiency correction is done by simulating many events according to a model where the decay $\Lambda_{b}^{0} \rightarrow J / \psi K^{-} p$ has a unit matrix element and so the decay distribution merely represents the available phase space. Then after the fully simulated events are reconstructed maps of the efficiencies versus all of the variables are formed. An unbinned maximum liklihood $(\mathcal{L})$ fit is then performed. The differences between values of $-2 \log \mathcal{L}$ are used to discriminate among the fits with various resonant or non-resonant components included. The liklihood $\mathcal{L}$ is itself a function of $m_{K p}$ and the five angular variables.

The results with only $\Lambda^{*}$ without any $P_{c}^{+}$component are shown in Fig. 6. The $m_{K P}$ variable is one of the fit variables, while $m_{J / \psi p}$ is calculated from the other variables. The $m_{K p}$ distribution is reasonably well described by $\Lambda^{*}$ resonances and their interferences, however the peaking structure in $m_{J / \psi p}$ is not reproduced.

Not satisfied with using all the known $\Lambda^{*}$ states LHCb tried several other different configurations: (i) added all the possible $\Sigma^{*}$ states, (ii) added two additional $\Lambda^{*}$ allowing their masses and widths to float in the fit and allowed spins up to $5 / 2$ with both parities, and (iii) added four non-resonant components with $J^{P}=1 / 2^{+}, 1 / 2^{-}, 3 / 2^{+}$, and $3 / 2^{-}$. None of these fits explains the data, indeed the improvements were small.

\subsubsection{The matrix element for $\Lambda_{b}^{0} \rightarrow J / \psi P_{c}^{+} K^{-}$decay}

In order to examine whether resonant structure can explain the angular data and the $J / \psi p$ mass spectrum the matrix element for the decay $\Lambda_{b}^{0} \rightarrow J / \psi P_{c}^{+} K^{-}, P_{c}^{+} \rightarrow J / \psi p$ needs to be constructed. The decay angles for the $P_{c}^{+}$decay sequence are defined in Fig. 7. The matrix element for the $P_{c}^{+}$decay chain is given by

$$
\begin{aligned}
\mathcal{M}_{\lambda_{\Lambda_{b}^{0}}, \lambda_{p}^{P_{c}}, \Delta \lambda_{\mu}^{P_{c}}}^{P_{c}} \equiv & \sum_{j} \sum_{\lambda_{P_{c}}} \sum_{\lambda_{\psi}^{P_{c}}} \mathcal{H}_{\lambda_{P_{c}}, 0}^{\Lambda_{b}^{0} \rightarrow P_{c j} K} D_{\lambda_{\Lambda_{b}^{0}}, \lambda_{P_{c}}}^{\frac{1}{2}}\left(\phi_{P_{c}}, \theta_{\Lambda_{b}^{0}}^{P_{c}}, 0\right)^{*} \\
& \times \mathcal{H}_{\lambda_{\psi}^{P_{c}}, \lambda_{p}^{P_{c}}}^{P_{c j} \rightarrow \psi p} D_{\lambda_{P_{c}}, \lambda_{\psi}^{P_{c}}-\lambda_{p}^{P_{c}}}^{J_{P_{P_{c}}}}\left(\phi_{\psi}, \theta_{P_{c}}, 0\right)^{*} R_{P_{c j}}\left(m_{\psi p}\right) \\
& \times D_{\lambda_{\psi}^{P_{c}}, \Delta \lambda_{\mu}^{P_{c}}}^{1}\left(\phi_{\mu}^{P_{c}}, \theta_{\psi}^{P_{c}}, 0\right)^{*},
\end{aligned}
$$

where the angles and helicity states carry the superscript or subscript $P_{c}$ to distinguish them from those defined for the $\Lambda^{*}$ decay chain. The sum over $j$ allows for the possibility of contributions from more than one $P_{c}^{+}$resonance. There are $2(3)$ independent helicity couplings $\mathcal{H}_{\lambda_{\psi}^{P_{c}}, \lambda_{p}^{P_{c}}}^{P_{p} \rightarrow \psi p}$ for $J_{P_{c j}}=\frac{1}{2}\left(>\frac{1}{2}\right)$, and a ratio of the two $\mathcal{H}_{\lambda_{P_{c}}, 0}^{\Lambda_{b}^{0} \rightarrow P_{c j} K}$ couplings, to determine from the data.
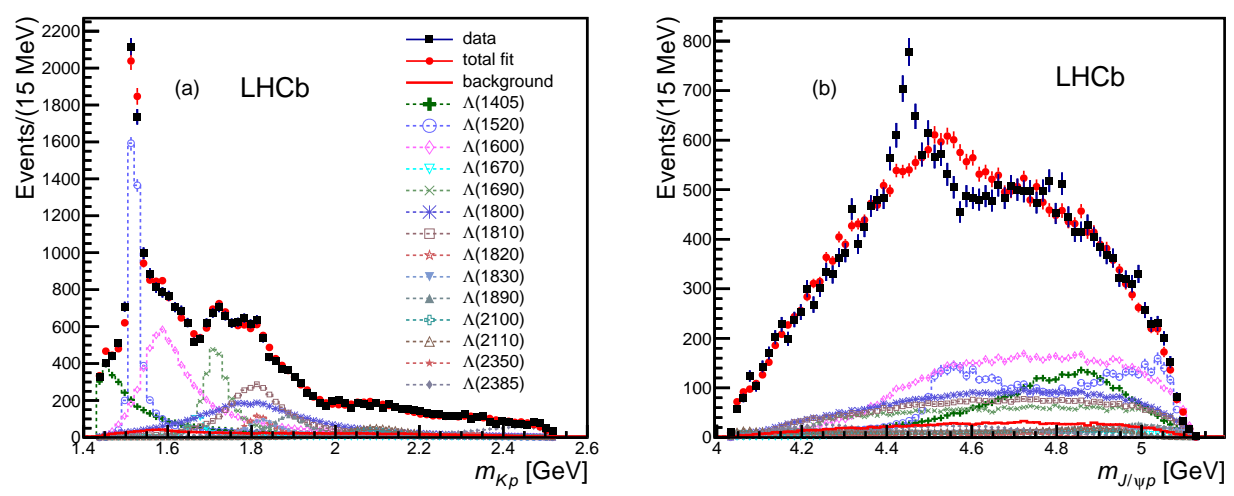

Figure 6: Results for (a) $m_{K p}$ and (b) $m_{J / \psi p}$ for the extended $\Lambda^{*}$ model fit without $P_{c}^{+}$states. The data are shown as (black) squares with error bars, while the (red) circles show the results of the fit. The error bars on the points showing the fit results are due to simulation statistics (from [11]). 


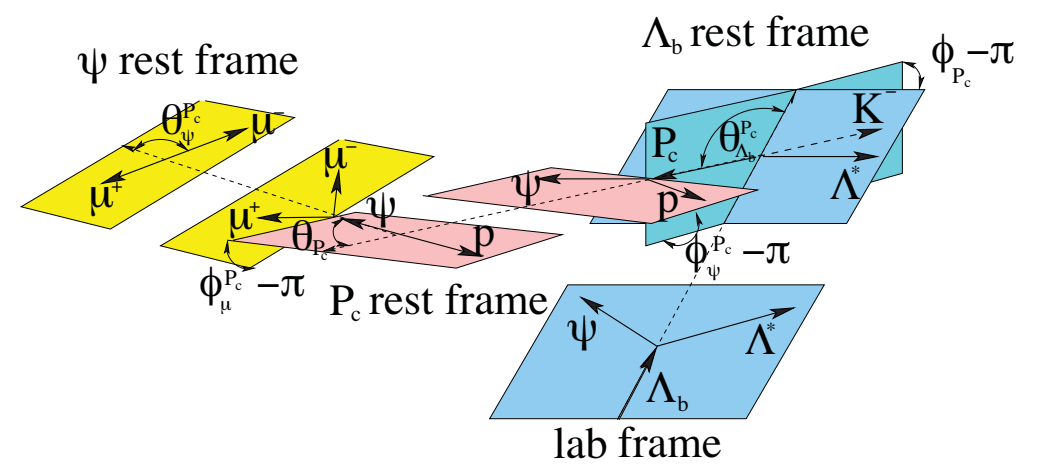

Figure 7: Definition of the decay angles in the $P_{c}^{+}$decay chain (from [11]).

Before the matrix elements for the two decay sequences can be added coherently, the proton and muon helicity states in the $\Lambda^{*}$ decay chain must be expressed in the basis of helicities in the $P_{c}^{+}$decay chain. The appropriate transformation is:

$$
|\mathcal{M}|^{2}=\sum_{\lambda_{\Lambda_{b}^{0}}} \sum_{\lambda_{p}} \sum_{\Delta \lambda_{\mu}}\left|\mathcal{M}_{\lambda_{\Lambda_{b}^{0}}^{\Lambda^{*}}, \lambda_{p}, \Delta \lambda_{\mu}}^{\Lambda^{*}}+e^{i \Delta \lambda_{\mu} \alpha_{\mu}} \sum_{\lambda_{p}^{P_{c}}} d_{\lambda_{p}^{P_{c}}, \lambda_{p}}^{\frac{1}{2}}\left(\theta_{p}\right) \mathcal{M}_{\lambda_{\Lambda_{b}^{0}}, \lambda_{p}^{P_{c}}, \Delta \lambda_{\mu}}^{P_{P}}\right|^{2}
$$

where $\theta_{p}$ is the polar angle in the $p$ rest frame between the boost directions from the $\Lambda^{*}$ and $P_{c}^{+}$rest frames, and $\alpha_{\mu}$ is the azimuthal angle correcting for the difference between the muon helicity states in the two decay chains. Note that $m_{\psi p}, \theta_{\Lambda_{b}^{0}}^{P_{c}}, \phi_{P_{c}}, \theta_{P_{c}}, \phi_{\psi}, \theta_{\psi}^{P_{c}}, \phi_{\mu}^{P_{c}}, \theta_{p}$ and $\alpha_{\mu}$ can all be derived from the values of $m_{K p}$ and $\Omega$, and thus do not constitute independent dimensions in the $\Lambda_{b}^{0}$ decay phase space.

$\Lambda_{b}^{0}$ production at the LHC is mediated by strong interactions which conserve parity and, therefore, cannot produce longitudinal $\Lambda_{b}^{0}$ polarization $\left[101\right.$. Thus, $\lambda_{\Lambda_{b}^{0}}=+1 / 2$ and $-1 / 2$ values are equally likely, which is assumed in writing down Eq. 114.

\subsubsection{Amplitude fits of $\Lambda_{b}^{0} \rightarrow J / \psi K^{-} p$ allowing $P_{c}^{+}$states}

In each fit we minimize $-2 \ln \mathcal{L}$ where $\mathcal{L}$ represents the fit likelihood. The difference of $\Delta \equiv-2 \ln \mathcal{L}$ between different amplitude models reflects the goodness of fit. For two models representing separate hypotheses, e.g. when discriminating between different $J^{P}$ values assigned to a $P_{c}^{+}$state, the assumption of a $\chi^{2}$ distribution with one degree of freedom for $\Delta$ under the disfavored $J^{P}$ hypothesis allows the calculation of a lower limit on the significance of its rejection, i.e. the p-value [102]. Therefore, it is convenient to express values of $\Delta$ as $n_{\sigma}^{2}$, where $n_{\sigma}$ corresponds to the number of standard deviations in the normal distribution with the same p-value. When discriminating between models without and with $P_{c}^{+}$states, $n_{\sigma}$ overestimates the p-value by a modest amount. Thus, we use simulations to obtain better estimates of the significance of the $P_{c}^{+}$states.

We perform separate fits for $J^{P}$ values of $1 / 2^{ \pm}, 3 / 2^{ \pm}$and $5 / 2^{ \pm}$. The mass and width of the putative $P_{c}^{+}$state are allowed to vary. The best fit prefers a $5 / 2^{+}$state, which improves $-2 \ln \mathcal{L}$ by $14.7^{2}$. Figure 8 shows the projections for this fit. While the $m_{K p}$ projection is well described, clear discrepancies in $m_{J / \psi p}$ remain visible.

The next step is to fit with two $P_{c}^{+}$states including their allowed interference. These fits were performed both with the reduced model and the extended model in order to estimate systematic uncertainties. Toy simulations are done to more accurately evaluate the statistical significances of the two states, resulting in 9 and 12 standard deviations, for lower mass and higher mass states, using the extended model which gives lower significances. The best fit projections are shown in Fig. 9. Both $m_{K p}$ and the peaking structure in $m_{J / \psi p}$ are reproduced by the fit. The reduced model has 64 free parameters 

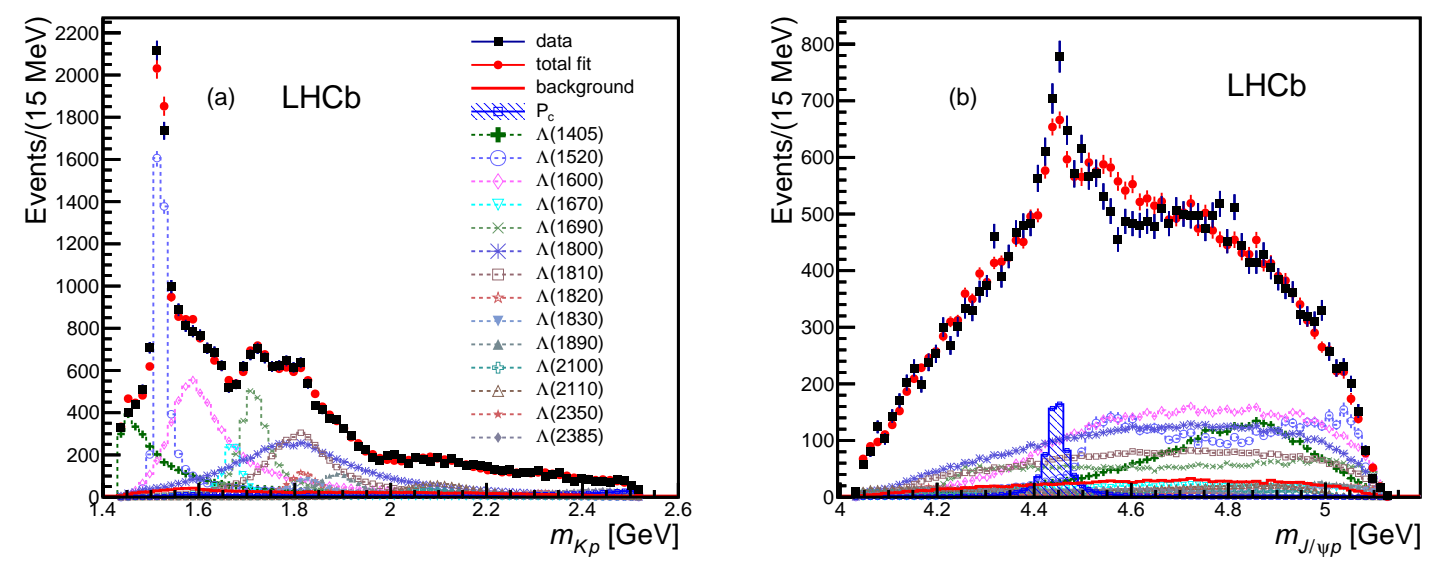

Figure 8: Results of the fit with one $J^{P}=5 / 2^{+} P_{c}^{+}$candidate. (a) Projection of the invariant mass of $K^{-} p$ combinations from $\Lambda_{b}^{0} \rightarrow J / \psi K^{-} p$ candidates. The data are shown as (black) squares with error bars, while the (red) circles show the results of the fit; (b) the corresponding $J / \psi p$ mass projection. The (blue) shaded plot shows the $P_{c}^{+}$projection, the other curves represent individual $\Lambda^{*}$ states. From [11].

for the $\Lambda^{*}$ rather than 146 and allows for a much more efficient examination of the parameter space and, thus, is used for numerical results. The two $P_{c}^{+}$states are found to have masses of $4380 \pm 8 \pm 29 \mathrm{MeV}$ and $4449.8 \pm 1.7 \pm 2.5 \mathrm{MeV}$, with corresponding widths of $205 \pm 18 \pm 86 \mathrm{MeV}$ and $39 \pm 5 \pm 19 \mathrm{MeV}$. (Whenever two uncertainties are quoted the first is statistical and the second systematic.) The fractions of the total sample due to the lower mass and higher mass states are $(8.4 \pm 0.7 \pm 4.2) \%$ and $(4.1 \pm 0.5 \pm 1.1) \%$, respectively. The overall branching fraction has recently be determined to be [103].

$$
\mathcal{B}\left(\Lambda_{b}^{0} \rightarrow J / \psi K^{-} p\right)=\left(3.04 \pm 0.04_{-0.43}^{+0.55}\right) \times 10^{-4}
$$

where the systematic uncertainty is largely due to the normalization procedure, leading to the product
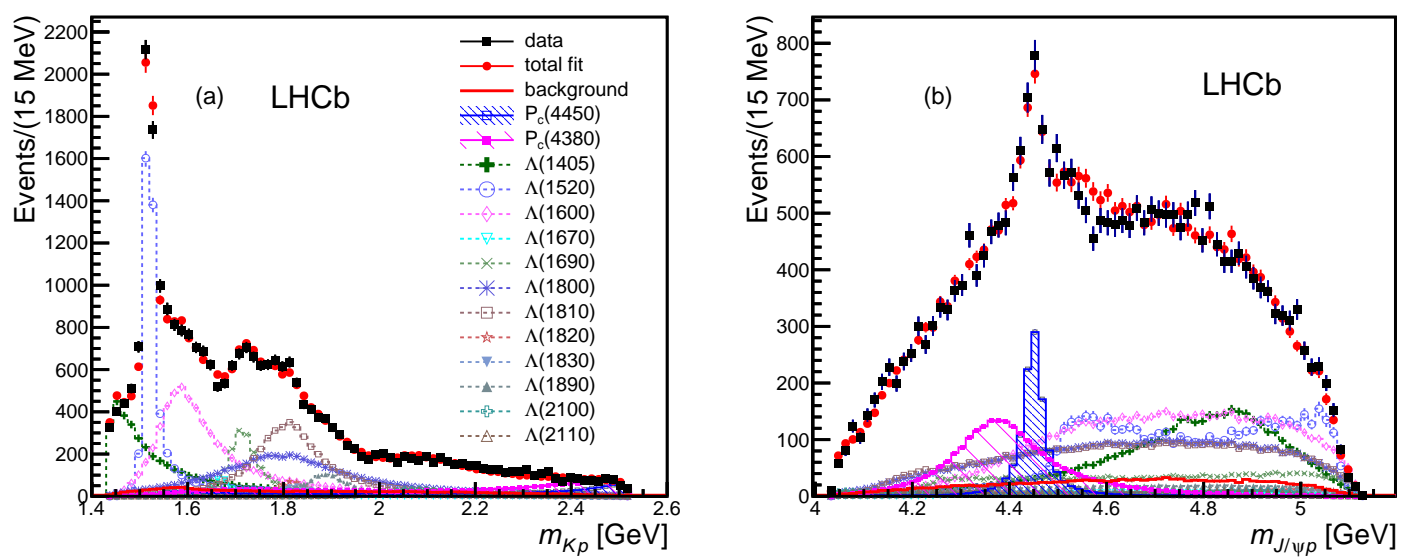

Figure 9: Fit projections for (a) $m_{K p}$ and (b) $m_{J / \psi p}$ for the reduced $\Lambda^{*}$ model with two $P_{c}^{+}$states (see Table 2). The data are shown as solid (black) squares, while the solid (red) points show the results of the fit. The solid (red) histogram shows the background distribution. The (blue) open squares with the shaded histogram represent the $P_{c}(4450)^{+}$state, and the shaded histogram topped with (purple) filled squares represents the $P_{c}(4380)^{+}$state. Each $\Lambda^{*}$ component is also shown (from [11]). 
branching fractions:

$$
\begin{aligned}
& \mathcal{B}\left(\Lambda_{b}^{0} \rightarrow P_{c}(4380)^{+} K^{-} p\right) \mathcal{B}\left(P_{c}(4380)^{+} \rightarrow J / \psi p\right)=\left(2.56_{-1.34}^{+1.38}\right) \times 10^{-5} \\
& \mathcal{B}\left(\Lambda_{b}^{0} \rightarrow P_{c}(4450)^{+} K^{-} p\right) \mathcal{B}\left(P_{c}(4450)^{+} \rightarrow J / \psi p\right)=\left(1.25_{-0.40}^{+0.42}\right) \times 10^{-5}
\end{aligned}
$$

where all the uncertainties have been added in quadrature.

The best fit solution has spin-parity $J^{P}$ values of $\left(3 / 2^{-}, 5 / 2^{+}\right)$. Acceptable solutions are also found for additional cases with opposite parity, either $\left(3 / 2^{+}, 5 / 2^{-}\right)$or $\left(5 / 2^{+}, 3 / 2^{-}\right)$. The five angular distributions are also well fit as can be seen in Fig. 10.

The fit projections in different slices of $K^{-} p$ invariant mass are given in Fig. 11. In slice (a) the $P_{c}^{+}$ states are not present, nor should they be as they are outside of the Dalitz plot boundary. In slice $(d)$ both $P_{c}^{+}$states form a large part of the mass spectrum; there is also a considerable amount of negative interference between them. This can be seen better by examining the decay angle of the $P_{c}^{+}, \theta_{P}$, the angle of the proton in $J / \psi p$ rest frame with respect to the $P_{c}^{+}$direction transformed into its rest frame, shown in Fig. 12 for the entire $m_{K p}$ range. The summed fit projections agrees very well with the angular distributions in the data showing that two interfering states are needed to reproduce the asymmetric distribution 5

Systematic uncertainties are evaluated for the masses, widths and fit fractions of the $P_{c}^{+}$states, and for the fit fractions of the two lightest and most significant $\Lambda^{*}$ states. Additional sources of modeling uncertainty that were not considered may affect the fit fractions of the heavier $\Lambda^{*}$ states. The sources of systematic uncertainties are listed in Table 3. They include differences between the results of the extended versus reduced model, varying the $\Lambda^{*}$ masses and widths, uncertainties in the identification

\footnotetext{
${ }^{5}$ It can be shown mathematically that the states need to be of opposite parity.
}

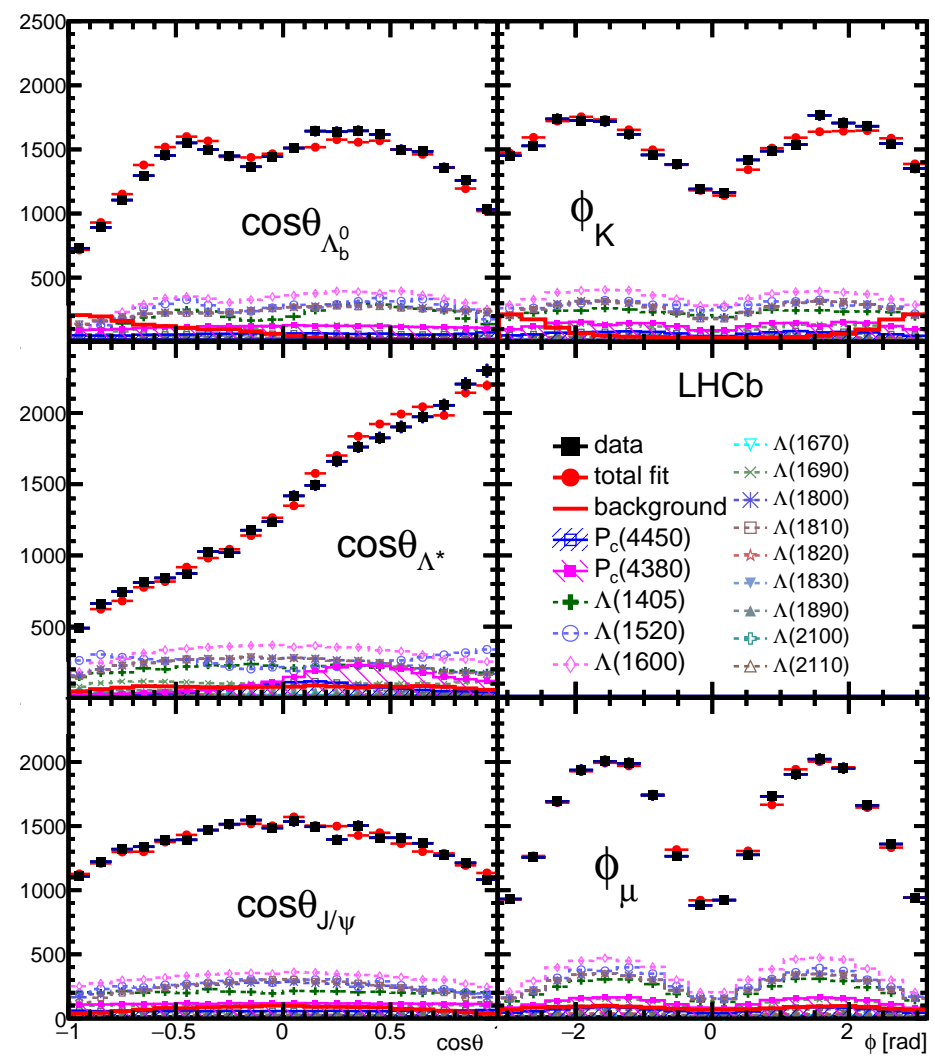

Figure 10: Various decay angular distributions for the fit with two $P_{c}^{+}$states. The data are shown as (black) squares, while the (red) circles show the results of the fit. Each fit component is also shown. The angles are defined in the text (from [11]). 


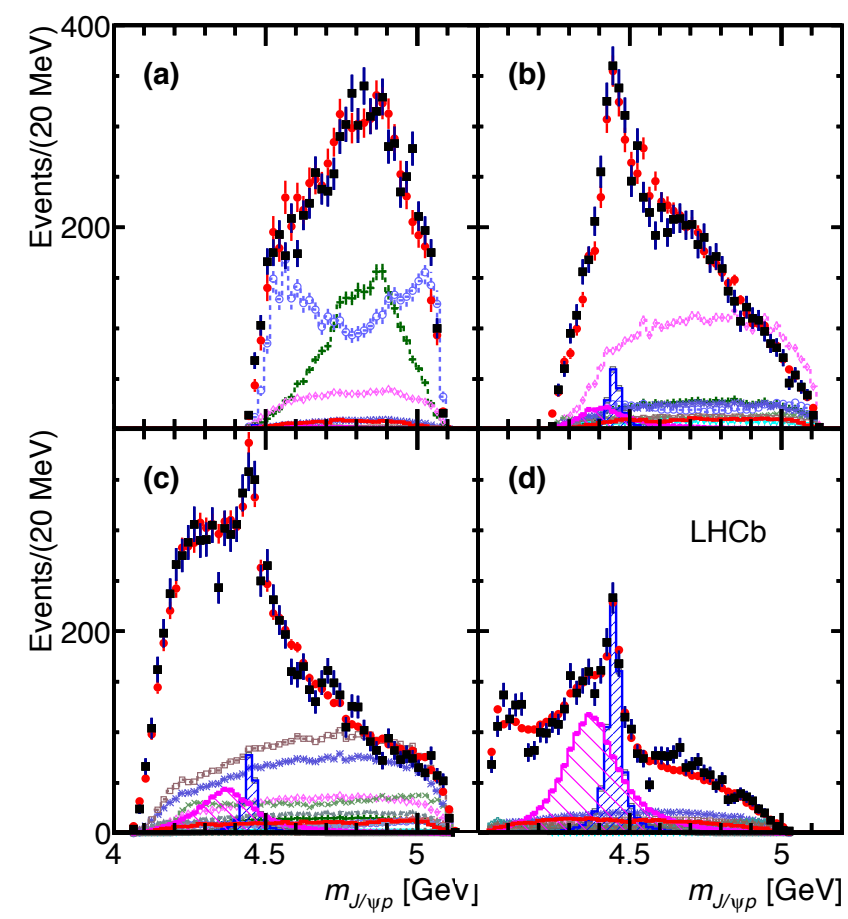

Figure 11: $m_{J / \psi p}$ in various intervals of $m_{K p}$ for the fit with two $P_{c}^{+}$states: (a) $m_{K p}<1.55 \mathrm{GeV}$, (b) $1.55<m_{K p}<1.70 \mathrm{GeV}$, (c) $1.70<m_{K p}<2.00 \mathrm{GeV}$, and (d) $m_{K p}>2.00 \mathrm{GeV}$. The data are shown as (black) squares with error bars, while the (red) circles show the results of the fit. The blue and purple histograms show the two $P_{c}^{+}$states. See Fig. 10 for the legend (from [11]).

requirements for the proton, and restricting its momentum, inclusion of a nonresonant amplitude in the fit, use of separate higher and lower $\Lambda_{b}^{0}$ mass sidebands, alternate $J^{P}$ fits, varying the Blatt-Weisskopf barrier factor, $d$, between 1.5 and $4.5 \mathrm{GeV}^{-1}$ in the Breit-Wigner mass shape-function, changing the angular momentum $L$ by one or two units, and accounting for potential mis-modeling of the efficiencies. For the 1 (1405) fit fraction we also added an uncertainty for the Flatté couplings, determined by both halving and doubling their ratio, and taking the maximum deviation as the uncertainty.

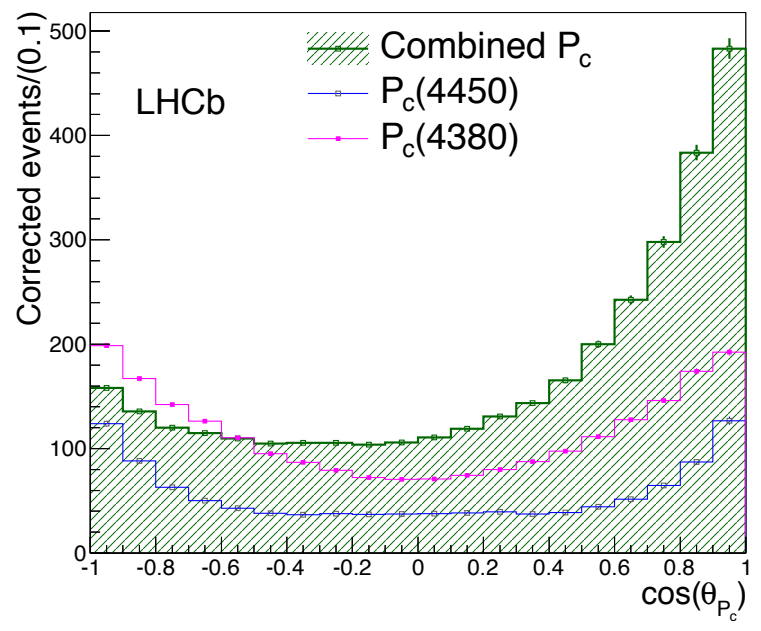

Figure 12: Efficiency corrected and background subtracted fit projections of the decay angular distributions for the two $P_{c}^{+}$states and their sum. Values of $\cos \theta_{P_{c}}$ near -1 are correlated with values of $m_{K p}$ near threshold, while those near +1 are correlated with higher values (from [104]). 
Table 3: Summary of systematic uncertainties on $P_{c}^{+}$masses, widths and fit fractions, and $\Lambda^{*}$ fit fractions. A fit fraction is the ratio of the phase space integrals of the matrix element squared for a single resonance and for the total amplitude. The terms "low" and "high" correspond to the lower and higher mass $P_{c}^{+}$states.

\begin{tabular}{|c|c|c|c|c|c|c|c|c|}
\hline \multirow[t]{2}{*}{ Source } & \multicolumn{2}{|c|}{$M_{0}(\mathrm{MeV})$} & \multicolumn{4}{|c|}{$\Gamma_{0}(\mathrm{MeV})$} & \multicolumn{2}{|c|}{ Fit fractions (\%) } \\
\hline & low & high & low & high & low & high & $\Lambda(1405)$ & $\Lambda(1520)$ \\
\hline Extended vs. reduced & 21 & 0.2 & 54 & 10 & 3.14 & 0.32 & 1.37 & 0.15 \\
\hline$\Lambda^{*}$ masses \& widths & 7 & 0.7 & 20 & 4 & 0.58 & 0.37 & 2.49 & 2.45 \\
\hline Proton ID & 2 & 0.3 & 1 & 2 & 0.27 & 0.14 & 0.20 & 0.05 \\
\hline $10<p_{p}<100 \mathrm{GeV}$ & 0 & 1.2 & 1 & 1 & 0.09 & 0.03 & 0.31 & 0.01 \\
\hline Nonresonant & 3 & 0.3 & 34 & 2 & 2.35 & 0.13 & 3.28 & 0.39 \\
\hline Separate sidebands & 0 & 0 & 5 & 0 & 0.24 & 0.14 & 0.02 & 0.03 \\
\hline$J^{P}\left(3 / 2^{+}, 5 / 2^{-}\right)$or $\left(5 / 2^{+}, 3 / 2^{-}\right)$ & 10 & 1.2 & 34 & 10 & 0.76 & 0.44 & & \\
\hline$d=1.5-4.5 \mathrm{GeV}^{-1}$ & 9 & 0.6 & 19 & 3 & 0.29 & 0.42 & 0.36 & 1.91 \\
\hline$L_{\Lambda_{b}^{0}}^{P^{+}} \Lambda_{b}^{0} \rightarrow P_{c}^{+}($low $/$high $) K^{-}$ & 6 & 0.7 & 4 & 8 & 0.37 & 0.16 & & \\
\hline$L_{P_{c}^{+}}^{+} P_{c}^{+}($low $/$high $) \rightarrow J / \psi p$ & 4 & 0.4 & 31 & 7 & 0.63 & 0.37 & & \\
\hline$L_{\Lambda_{b}^{0}}^{\Lambda_{n}^{*}} \Lambda_{b}^{0} \rightarrow J / \psi \Lambda^{*}$ & 11 & 0.3 & 20 & 2 & 0.81 & 0.53 & 3.34 & 2.31 \\
\hline Efficiencies & 1 & 0.4 & 4 & 0 & 0.13 & 0.02 & 0.26 & 0.23 \\
\hline Change $\Lambda(1405)$ coupling & 0 & 0 & 0 & 0 & 0 & 0 & 1.90 & 0 \\
\hline Overall & 29 & 2.5 & 86 & 19 & 4.21 & 1.05 & 5.82 & 3.89 \\
\hline
\end{tabular}

The stability of the results are cross-checked by comparing the data recorded in 2011/2012, with the LHCb dipole magnet polarity in up/down configurations, $\Lambda_{b}^{0} / \bar{\Lambda}_{b}^{0}$ decays, and $\Lambda_{b}^{0}$ produced with low/high values of $p_{\mathrm{T}}$. The fitters were tested on simulated pseudoexperiments and no biases were found. In addition, selection requirements are varied, and the vetoes of $\bar{B}_{s}^{0}$ and $\bar{B}^{0}$ are removed and explicit models of those backgrounds added to the fit; all give consistent results.

Further evidence for the resonant character of the higher mass, narrower, $P_{c}^{+}$state is obtained by viewing the evolution of the complex amplitude in the Argand diagram [12]. In the amplitude fits discussed above, the $P_{c}(4450)^{+}$is represented by a Breit-Wigner amplitude, where the magnitude and phase vary with $m_{J / \psi p}$ according to an approximately circular trajectory in the $\left(\operatorname{Re} A^{P_{c}}, \operatorname{Im} A^{P_{c}}\right)$ plane, where $A^{P_{c}}$ is the $m_{J / \psi p}$ dependent part of the $P_{c}(4450)^{+}$amplitude. An additional fit to the data was performed using the reduced $\Lambda^{*}$ model, in which we represent the $P_{c}(4450)^{+}$amplitude as the combination of independent complex amplitudes at six equidistant points from $-\Gamma$ to $\Gamma$ around $M=4449.8 \mathrm{MeV}$ as determined in the default fit. Real and imaginary parts of the amplitude are interpolated in mass between the fitted points. The resulting Argand diagram, shown in Fig. 13(a), is consistent with a rapid counter-clockwise change of the $P_{c}(4450)^{+}$phase when its magnitude reaches the maximum, a behavior characteristic of a resonance. A similar study for the wider state is shown in Fig. 13(b); although the fit does show a large phase change, the amplitude values are sensitive to the details of the $\Lambda^{*}$ model and so this latter study is not conclusive.

\subsection{Consistency check using $\Lambda_{b}^{0} \rightarrow J / \psi p \pi^{-}$decays}

The $\Lambda_{b}^{0} \rightarrow J / \psi p \pi^{-}$decay is the Cabibbo suppressed version of the $\Lambda_{b}^{0} \rightarrow J / \psi K^{+} \pi^{-}$decay. Figure 15 shows the quark level diagrams for both the decay into (a) $J / \psi N^{*}$ and (b) $P_{c}^{+} \pi^{-}$. (Compare with Fig. 4.) Also note that since there are two $d$ quarks in the final state, the origin of the two $d$ quarks 


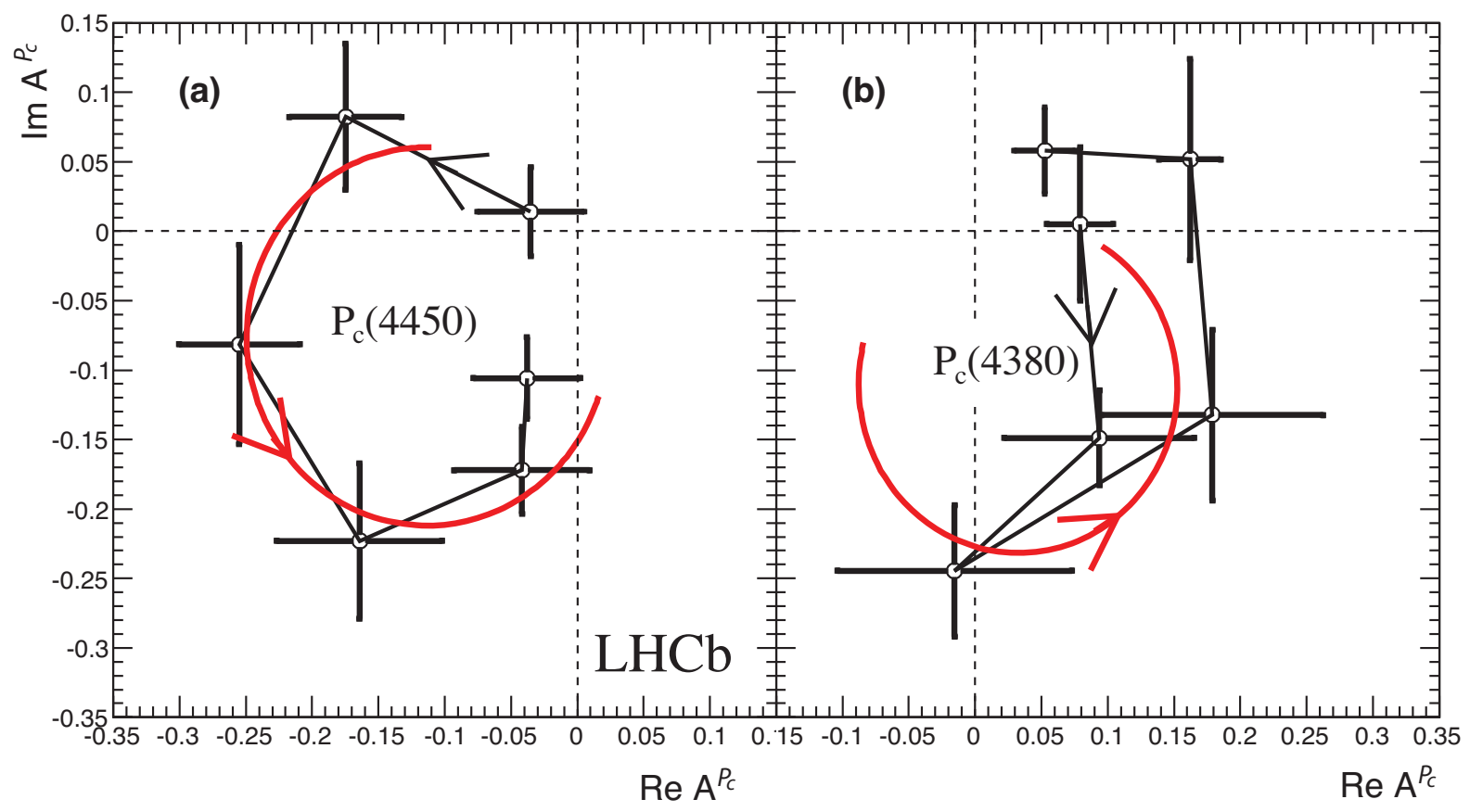

Figure 13: Fitted values of the real and imaginary parts of the amplitudes for the baseline $\left(3 / 2^{-}\right.$, $\left.5 / 2^{+}\right)$fit for a) the $P_{c}(4450)^{+}$state and b) the $P_{c}(4380)^{+}$state, each divided into six $m_{J / \psi p}$ bins of equal width between $-\Gamma$ and $+\Gamma$ shown in the Argand diagrams as connected points with error bars $\left(m_{J / \psi_{p}}\right.$ increases counterclockwise). The solid (red) curves are the predictions from the Breit-Wigner formula for the same mass ranges with $M(\Gamma)$ of 4450 (39) $\mathrm{MeV}$ and 4380 (205) $\mathrm{MeV}$, respectively, with the phases and magnitudes at the resonance masses set to the average values between the two points around $M$. The phase convention is fixed by the $1(1520)$. Systematic uncertainties are not included (from [1]).

can be switched, which corresponds to a different amplitude shown in (c), and these amplitudes can interfere and modify the decay rate 105]. The relative branching fractions of the suppressed versus non-suppressed modes has been measured as $\mathcal{B}\left(\Lambda_{b}^{0} \rightarrow J / \psi p \pi^{-}\right) / \mathcal{B}\left(\Lambda_{b}^{0} \rightarrow J / \psi p K^{-}\right)=0.0824 \pm 0.0024 \pm$ 0.0042 [106], consistent with the expectation of $\sim 0.05$, however, the crucial question is if we can see the pentaquark states at a rate consistent with this Cabibbo suppression. There is also the possibility of production of the exotic meson state $Z_{c}(4200)^{-}$that decays into $J / \psi \pi^{-}$; the Feynman diagram is shown in (d). The analogous concern, i.e. of a $J / \psi K^{-}$resonant system in the $\Lambda_{b}^{0} \rightarrow J / \psi K^{+} \pi^{-}$decay, was not present because the data showed the absence of such a reasonant state, and because it has not been seen in any other decay.

Event candidates are reconstructed with similar criteria as for the $J / \psi K^{-} \pi^{+}$mode with different particle identification criteria for the pion. Due to the larger backgrounds veto's are employed to get rid of backgrounds caused by proton particle identification failures including the modes $\bar{B}^{0} \rightarrow J / \psi \pi^{+} K^{-}$ or as $\bar{B}_{s}^{0} \rightarrow J / \psi K^{+} K^{-}$. Here the protons are interpreted as kaons and if the calculated invariant masses are within $\pm 3 \sigma$ of either of the known $B$ masses, the combination is rejected. In addition, $p \pi^{-}$ combinations within $\pm 5 \mathrm{MeV}$ of the $\Lambda$ mass are removed.

The invariant mass spectrum of $\Lambda_{b}^{0}$ candidates is shown in Fig. 14. The signal yield is $1885 \pm 50$. The signal is described by a double-sided Crystal Ball function [107]. The combinatorial background is modeled by an exponential function. The background shape of $\Lambda_{b}^{0} \rightarrow J / \psi p K^{-}$events is described by a histogram obtained from simulation; the normalization is not fixed, but allowed to vary in the fit. Candidates are assigned weights using the sPlot technique [108] allowing the signal and background 
components to be projected out depending on their $\mathrm{J} / \psi \mathrm{p} \pi^{-}$mass.

The analysis must incorporate all of the amplitudes shown in Fig. 15. The amplitude for diagram (a) is the same as that for $\Lambda_{b}^{0} \rightarrow J / \psi K^{-} p$ but with the final state $\Lambda^{*}$ states being replaced by $N^{*}$ states. These are listed in Table 4. Here two models are used, one called the Reduced Model (RM) which uses fewer angular momentum amplitudes and states and the Extended Model (EM). Table 2 lists the $N^{*}$ resonances considered in the amplitude model of $p \pi^{-}$contributions. There are 15 well-established $N^{*}$ resonances [12]. The high-mass and high-spin states $(9 / 2$ and $11 / 2)$ are not included, since they require $L \geq 3$ in the $\Lambda_{b}^{0}$ decay and therefore are unlikely to be produced near the upper kinematic limit. Included, however, are two unconfirmed high-mass, low-spin resonances found by the BESIII collaboration, the $N(2300)$ and the $N(2700)$ [109]. The amplitudes for diagrams (b) and (c) differ only by a sign [105] and thus are modeled as one amplitude for each of the $P_{c}^{+}$states. Finally, an amplitude is included for the possible $\Lambda_{b}^{0} \rightarrow Z_{c}(4200)^{-} p$ decay. ${ }^{6}$

Amplitude fits are performed by minimizing a six-dimensional unbinned negative log-likelihood, $-2 \ln \mathcal{L}$, with the background subtracted using the weights described above, and the efficiency folded into the signal probability density function, as discussed in detail in Ref. [11]. Shown in Fig. 16 are the results of fits to several different amplitude hypotheses to both the $p \pi^{-}$and $J / \psi p$ mass spectra. First let us concentrate on the fit where no exotic contributions are considered (amplitude (a) in Fig. 15), the one shown in the green open circles. The fit does describe the $p \pi^{-}$and $J / \psi p$ mass projections quite well. However, if one looks at the $p \pi^{-}$mass region above $1.8 \mathrm{GeV}$, which eliminates the "background" from the lower mass $N^{*}$ resonances, there is an excess of events in the $J / \psi p$ mass region between 4.2 and $4.5 \mathrm{GeV}$.

It is necessary to also consider the possibility of an exotic $J / \psi \pi^{-}$resonance. In Fig. 17 the plot of the $J / \psi \pi^{-}$invariant mass for the entire $m_{p \pi}$ and for $m_{p \pi}>1.8 \mathrm{GeV}$, shows an excess of events in the in the region between 4.1 and $4.3 \mathrm{GeV}$ compared with the fit using only the $J / \psi N^{*}$ model.

To test these other possibilities full amplitude fits including the decay angles are performed and differences in the $-2 \ln \mathcal{L}$ of fits computed. The baseline model includes the set of $\mathrm{RM} N^{*}$ resonances plus all three exotic states, labelled as "RM $N^{*}+Z_{c}+2 P_{c}$ " in the figures. The fit projections of all three exotic states are also shown. The $J / \psi \pi^{-}$mass projections are shown in Fig. 17.

\footnotetext{
${ }^{6}$ The construction of this amplitude is documented in the supplemental material of Ref. 11.
}

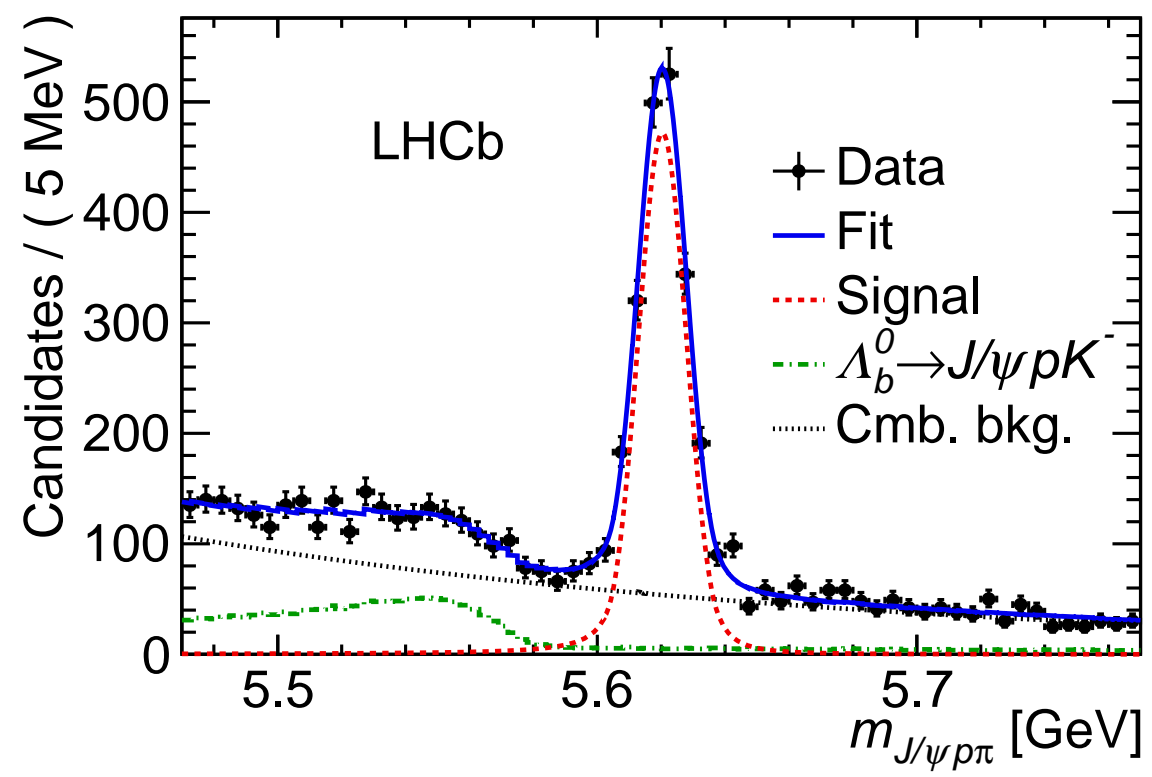

Figure 14: Invariant mass spectrum for the selected $\Lambda_{b}^{0} \rightarrow J / \psi p \pi^{-}$candidates (from 106]. 

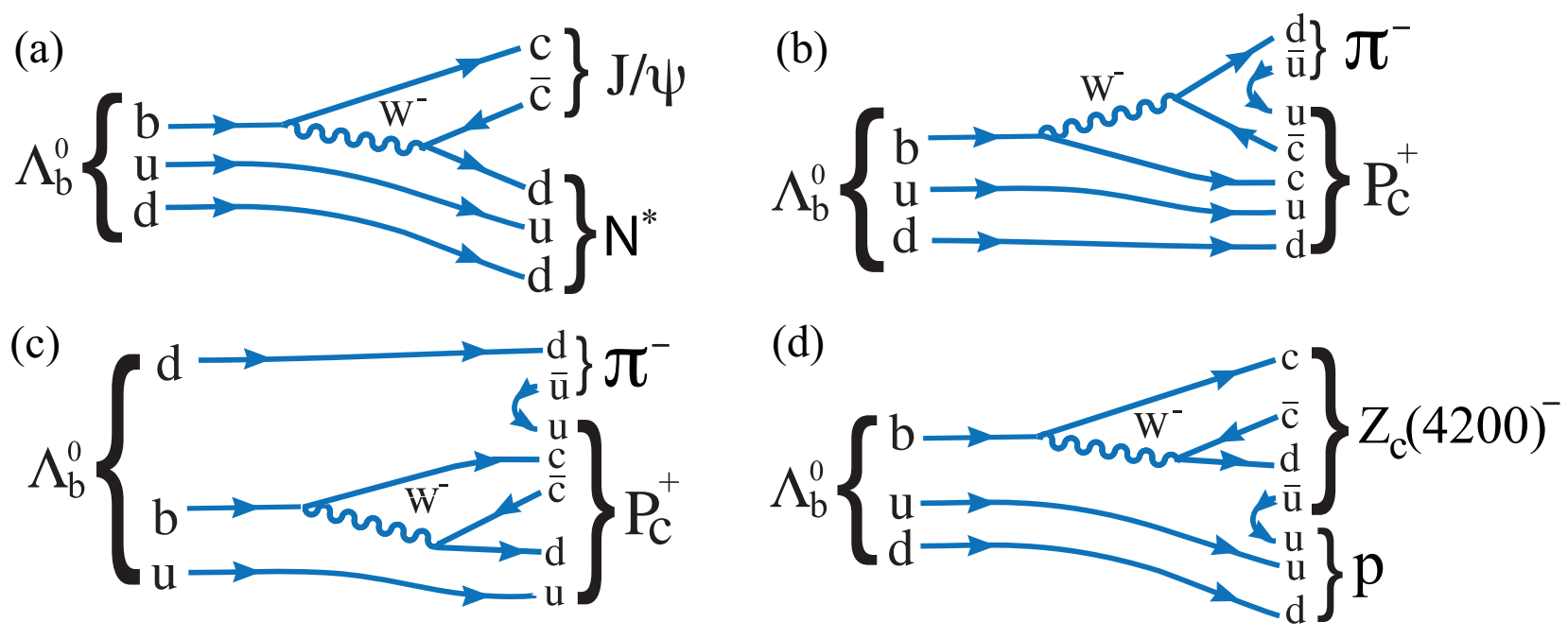

Figure 15: Feynman diagrams for (a) $\Lambda_{b}^{0} \rightarrow J / \psi N^{*}$, (b) $\Lambda_{b}^{0} \rightarrow P_{c}^{+} \pi^{-}$decay, (c) another diagram for $\Lambda_{b}^{0} \rightarrow P_{c}^{+} \pi^{-}$decay, and (d) diagram for $\Lambda_{b}^{0} \rightarrow Z_{c}(4200)^{-} p$ decay.

The fit with all three exotic states is superior to that of the only the $N^{*}$ model. Quantitatively, the change in $-2 \ln \mathcal{L}(\Delta(-2 \ln \mathcal{L}))$ corresponds to $3.9 \sigma$. Other models tried included using only the two $P_{c}$ states, or only the $Z_{c}$ state. Table 5 lists the models, the number of standard deviation $(\mathrm{N} \sigma)$ changes with respect to the $N^{*}$ EM only model, and the fit fractions.

The fit with either one of the two $P_{c}$ states, or only the $Z_{c}$ state can adequately describe the data, although the $Z_{c}$ only fit has a seemingly rather large fit fractions of $(17 \pm 3.5) \%$. Adding the $Z_{c}$ state hardly changes the $P_{c}$ fit fractions. These correspond to the ratio of branching fractions $\mathcal{B}\left(\Lambda_{b}^{0} \rightarrow\right.$ $\left.P_{c}(4380)^{+} \pi^{-}\right) / \mathcal{B}\left(\Lambda_{b}^{0} \rightarrow P_{c}(4380)^{+} K^{-}\right)=0.050 \pm 0.016_{-0.016}^{+0.026} \pm 0.025$ and $\mathcal{B}\left(\Lambda_{b}^{0} \rightarrow P_{c}(4450)^{+} \pi^{-}\right) / \mathcal{B}\left(\Lambda_{b}^{0} \rightarrow\right.$ $\left.P_{c}(4450)^{+} K^{-}\right)=0.033_{-0.014-0.010}^{+0.016+0.011} \pm 0.009$, where first uncertainty is statistical, the second systematic and the third due to the error on the fit fractions in the $J / \psi K^{-} p$ mode. These are consistent with the expectations for Cabibbo suppressed rates within the large uncertainties [105.

To summarize this section, the $J / \psi \pi^{-} p$ mode shows that the data are inconsistent with not having
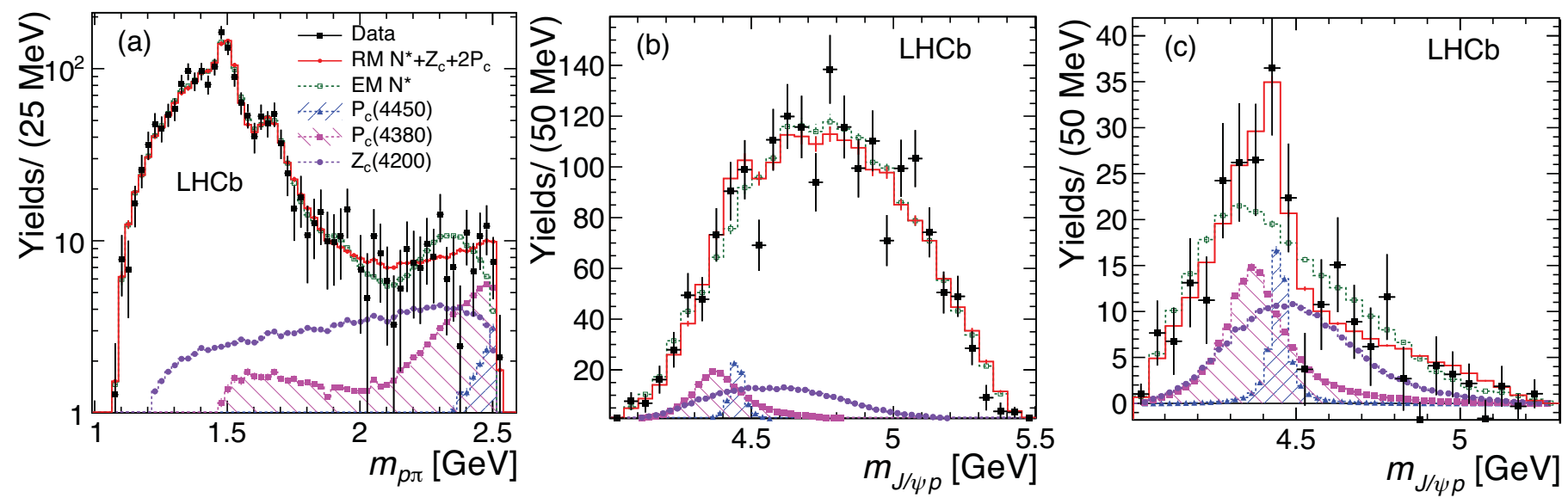

Figure 16: (a) Background-subtracted data and fit projections onto $m_{J / \psi p}$ for all events (log scale), (b) all events (linear scale), and (c) the $m_{p \pi}>1.8 \mathrm{GeV}$ region. The open circles (green) are for the $J / \psi N^{*}$ model using the EM suite of $N^{*}$ states, while the red histogram includes the RM sample of $N^{*}$ states as well as the two $P_{c}$ states and the $Z_{c}$ state; the other curves show the individual components (from [106]). 
Table 4: The $N^{*}$ resonances used in the different fits. Parameters are taken from the PDG [12]. The number of $L S$ couplings is listed in the columns to the right for the two versions Reduced Model (RM) and Extended Model (EM). To fix overall phase and magnitude conventions, the $N(1535)$ complex coupling of lowest $L S$ is set to $(1,0)$.

\begin{tabular}{lccccc} 
State & $J^{P}$ & Mass $(\mathrm{MeV})$ & Width $(\mathrm{MeV})$ & $\mathrm{RM}$ & EM \\
\hline $\mathrm{NR} p \pi$ & $1 / 2^{-}$ & - & - & 4 & 4 \\
$N(1440)$ & $1 / 2^{+}$ & 1430 & 350 & 3 & 4 \\
$N(1520)$ & $3 / 2^{-}$ & 1515 & 115 & 3 & 3 \\
$N(1535)$ & $1 / 2^{-}$ & 1535 & 150 & 4 & 4 \\
$N(1650)$ & $1 / 2^{-}$ & 1655 & 140 & 1 & 4 \\
$N(1675)$ & $5 / 2^{-}$ & 1675 & 150 & 3 & 5 \\
$N(1680)$ & $5 / 2^{+}$ & 1685 & 130 & - & 3 \\
$N(1700)$ & $3 / 2^{-}$ & 1700 & 150 & - & 3 \\
$N(1710)$ & $1 / 2^{+}$ & 1710 & 100 & - & 4 \\
$N(1720)$ & $3 / 2^{+}$ & 1720 & 250 & 3 & 5 \\
$N(1875)$ & $3 / 2^{-}$ & 1875 & 250 & - & 3 \\
$N(1900)$ & $3 / 2^{+}$ & 1900 & 200 & - & 3 \\
$N(2190)$ & $7 / 2^{-}$ & 2190 & 500 & - & 3 \\
$N(2300)$ & $1 / 2^{+}$ & 2300 & 340 & - & 3 \\
$N(2570)$ & $5 / 2^{-}$ & 2570 & 250 & - & 3 \\
\hline \multicolumn{5}{l}{ Free parameters } &
\end{tabular}

exotic components, are consistent with the expectations for the rates of the Cabibbo suppressed $P_{c}$ states, but also could be explained by an exotic $Z_{c}$ or both exotic contributions.

\subsection{Independent confirmation of the $\boldsymbol{P}_{c}(4450)^{+}$using a model independent $\Lambda^{*}$ description}

While a convincing case has been made for the existence of the two $P_{c}$ states, there is an alternative method of examining the consistency of the data with the presence of exotic states. The poorly under-
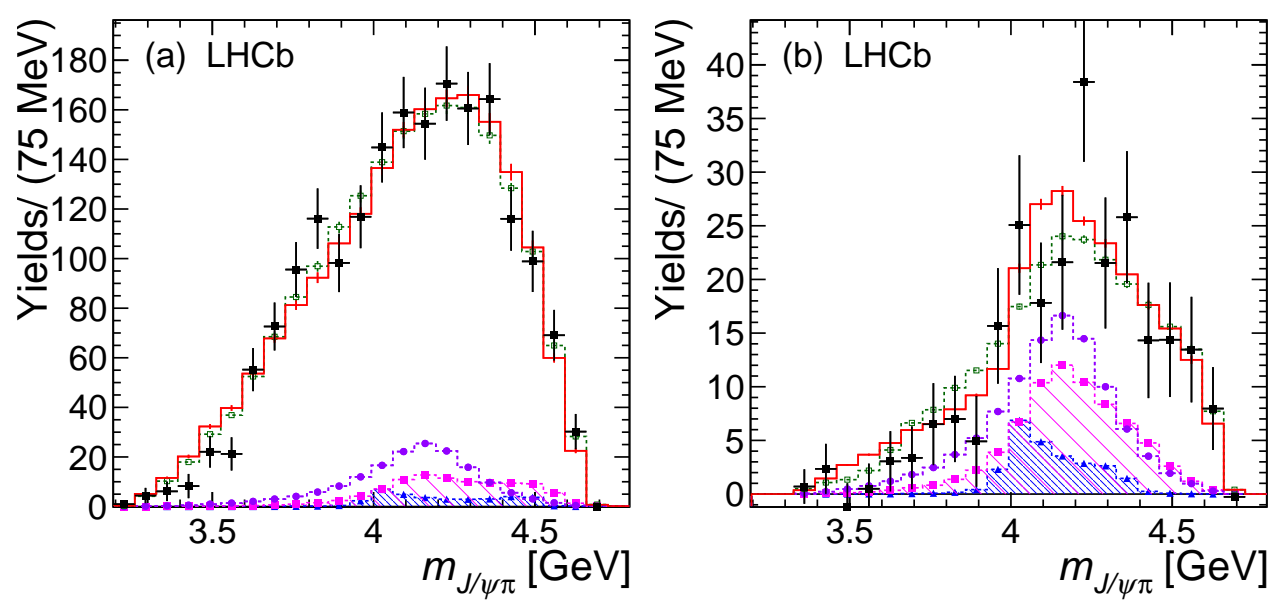

Figure 17: Background-subtracted data and fit projections onto $m_{J / \psi \pi}$ for (a) all events and (b) the $m_{p \pi}>1.8 \mathrm{GeV}$ region. See the legend and caption of Fig. 16 for a description of the components (from [106]). 
Table 5: Results of different amplitude model fits to the data. The change N $\sigma$ refers to the improvement in the fit with respect to the EM $N^{*}$ model. The fit fractions are the percentages of each of the final states as determined by the fit.

\begin{tabular}{lcccc} 
Model & $\mathrm{N} \sigma$ & \multicolumn{3}{c}{ Fit Fractions $(\%)$} \\
& & $P_{c}(4380)^{+}$ & $P_{c}(4450)^{+}$ & $Z_{c}(4200)^{-}$ \\
\hline$N^{*}+P_{c}+Z_{c}$ & 3.9 & $5.1 \pm 1.5_{-1.6}^{+2.6}$ & $1.6_{-0.6-0.5}^{+0.8+0.6}$ & $7.7 \pm 2.8_{-4.0}^{+3.4}$ \\
$N^{*}+P_{c}$ & 3.3 & $\approx 5.1$ & $\approx 1.6$ & - \\
$N^{*}+Z_{c}$ & 3.2 & - & - & $17.2 \pm 3.5$ \\
\hline
\end{tabular}

stood nature of the $\Lambda^{*}$ states [12] and the difficulty of knowing exactly which amplitudes are present can be circumvented by using moments related to the $\Lambda^{*}$ decay angle that can be calculated without knowledge of what $\Lambda^{*}$ states are present. The only assumption made is that very high spin states cannot be present in $\Lambda_{b}^{0} \rightarrow J / \psi \Lambda^{*}$ decays especially near the largest possible allowed $\Lambda^{*}$ masses. This approach was developed by the BaBar collaboration for their studies of $\left[110 \bar{B}^{0} \rightarrow J / \psi \pi^{-} K^{+}\right.$decays, where they attempted to explain the $Z(4430)^{-} \rightarrow J / \psi \pi^{-}$as the sum of interfering $K^{*}$ resonances. The same method was adapted by LHCb with opposite conclusions [111].

In principle, after integrating over the decay angular variables the decay amplitude can be written in terms of $m_{K p}$ and $m_{J / \psi p}$, or equivalently $m_{K p}$ and $\cos \theta_{\Lambda^{*}}$, where the latter is the helicity decay angle of the $K^{-} p$ system. For each bin in $m_{K p}$ the $\cos \theta_{\Lambda^{*}}$ distribution can be expressed as a sum over Legendre polynomials:

$$
d N / d \cos \theta_{\Lambda^{*}}=\sum_{\ell=0}^{\ell_{\max }}\left\langle P_{\ell}^{U}\right\rangle P_{\ell}\left(\cos \theta_{\Lambda^{*}}\right),
$$

where $N$ is the efficiency-corrected and background-subtracted signal yield, $\ell_{\max }$ is the maximum allowed angular momentum value, and $\left\langle P_{\ell}^{U}\right\rangle$ is an unnormalized Legendre moment of rank $\ell$,

$$
\left\langle P_{\ell}^{U}\right\rangle=\int_{-1}^{+1} d \cos \theta_{\Lambda^{*}} P_{\ell}\left(\cos \theta_{\Lambda^{*}}\right) d N / d \cos \theta_{\Lambda^{*}}
$$

To ensure the validity of this procedure, the event sample must be background subtracted and efficiency corrected over the full range of angular variables in the decay amplitude.

In this analysis 111 two hypotheses are considered, one where the $P_{c}^{+}$states are absent, denoted as $H_{0}$, and another where they are present, called $H_{1}$. For $H_{0}$ the maximum allowed value of $\ell_{\max }$ is determined from the angular momentum of the $\Lambda^{*}$. Since the $\Lambda^{*}$ decays into a spinless kaon and a spin $1 / 2$ proton, $\ell_{\max }$ can only be as large as twice the spin of any particular $\Lambda^{*}$ resonance.7 The maximum spin of these resonances are limited by the $\Lambda^{*}$ mass, with higher masses having larger allowed spins. This rather mild hypothesis is based on both experimental measurement and theoretical predictions as illustrated in Fig. 18.

This analysis takes advantage of the limited $\ell_{\max }\left(m_{K p}\right)$ by allowing only moments above the red line in Fig. 18. Thus the largest $\ell_{\max }$ to be considered is 9 and that is only for $m_{K p}>2.050 \mathrm{GeV}$. The background subtracted and efficiency corrected data projected in $m_{K p}$ are shown in Fig. 19.

A probability density function based on the $\Lambda^{*}$ helicity distribution is then formed via linear interpolation between the $m_{K p}$ bins, labeled as $k$, as

$$
\mathcal{P}\left(\cos \theta_{\Lambda^{*}} \mid H_{0}, m_{K p}{ }^{k}\right)=\sum_{l=0}^{\ell_{\max }\left(m_{K p}{ }^{k}\right)}\left\langle P_{\ell}^{N}\right\rangle^{k} P_{\ell}\left(\cos \theta_{\Lambda^{*}}\right) .
$$

\footnotetext{
${ }^{7}$ The angular distribution of a spin $\ell / 2$ resonance is described by a Legendre polynomial of order $\ell-1$ to which one more unit of additional angular momentum is possible due to the spin $1 / 2$ nature of the proton.
} 


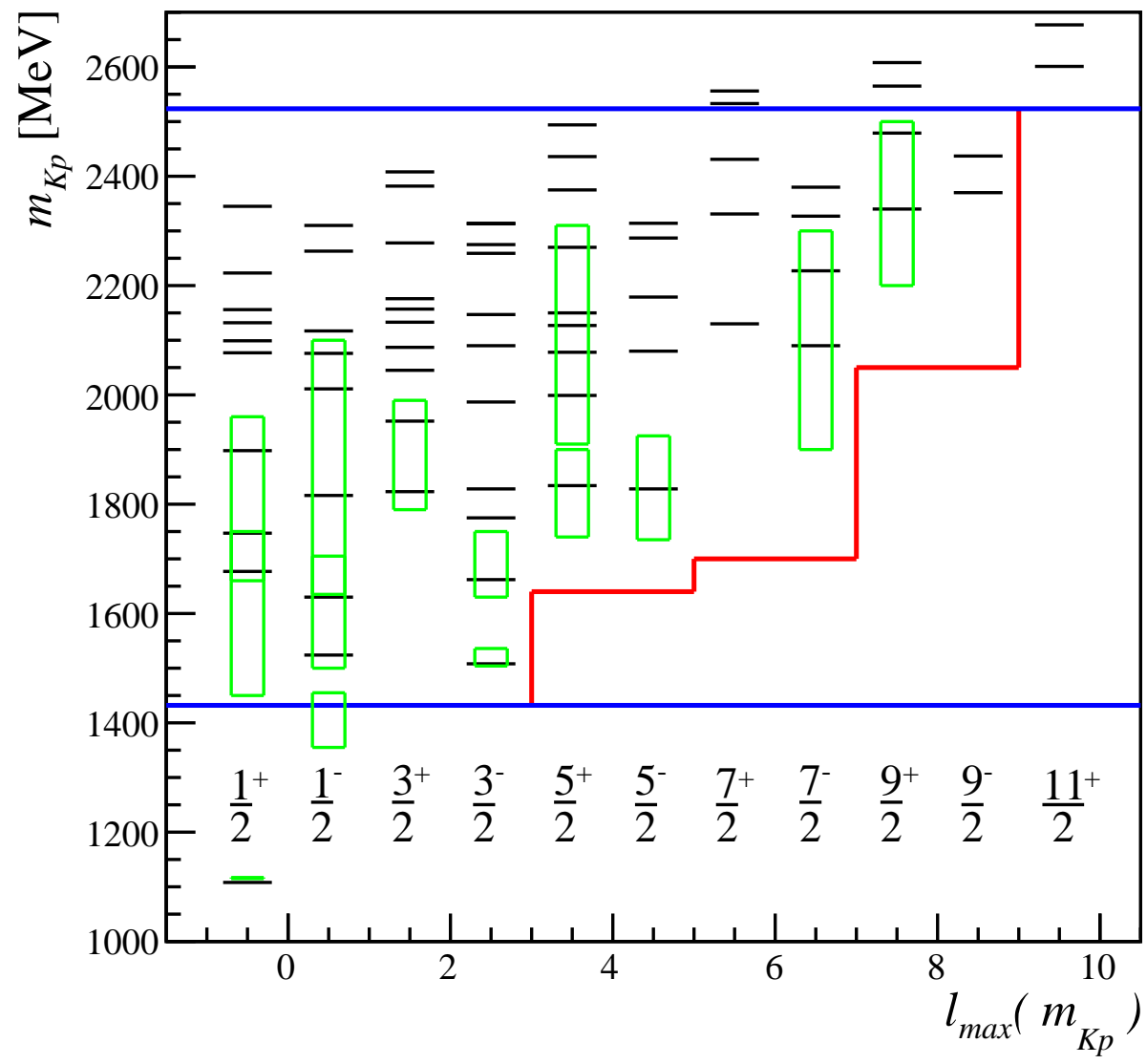

Figure 18: Excitations of the $\Lambda$ baryon. States predicted in ref. [112] are shown as short horizontal lines (black) and experimentally well-established $\Lambda^{*}$ states are shown as green boxes covering the mass ranges from $M_{0}-\Gamma_{0}$ to $M_{0}+\Gamma_{0}$. The $m_{K p}$ mass range probed in $\Lambda_{b}^{0} \rightarrow J / \psi p K^{-}$decays is shown by long horizontal lines (blue). The $\ell_{\max }$ upper limit as a function of $m_{K p}$ is shown as a stepped line (red). All contributions from $\Lambda^{*}$ states with $J^{P}$ values to the left of the red line are accepted by the filter (from 111).

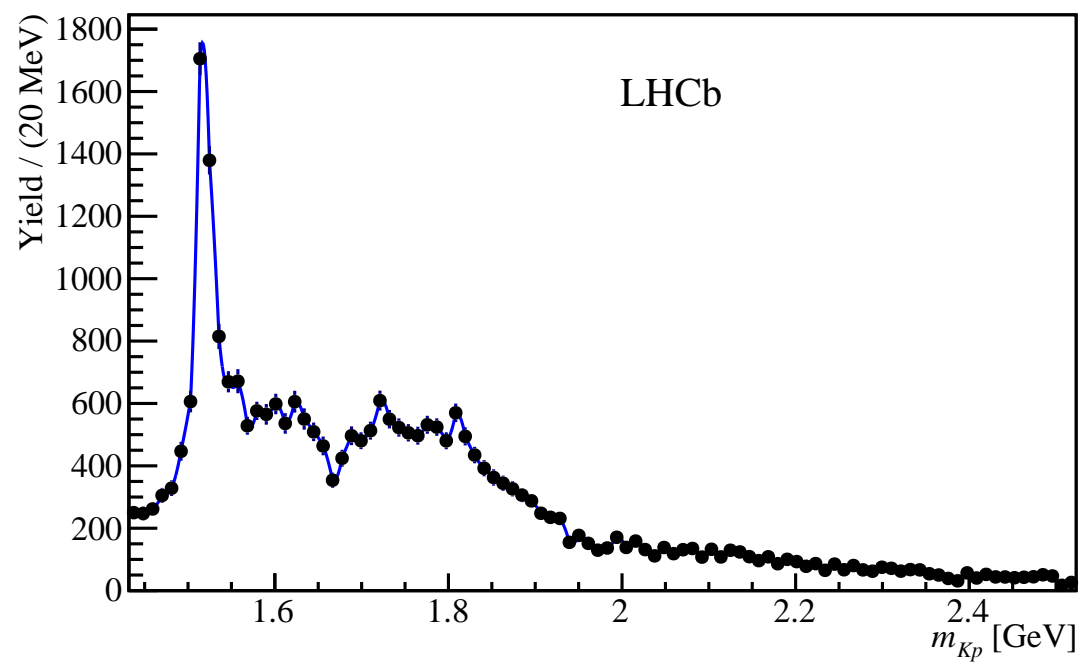

Figure 19: Efficiency-corrected and background-subtracted $m_{K p}$ distribution of the data (black points with error bars), with $\mathcal{P}\left(m_{K p} \mid H_{0}\right)$ superimposed (solid blue line). $\mathcal{P}\left(m_{K p} \mid H_{0}\right)$ fits the data by construction (from [111]). 
Here the Legendre moments $\left\langle P_{\ell}^{N}\right\rangle^{k}$ are normalized by the yield in the corresponding $m_{K p}$ bin. These data are used to determine

$$
\left\langle P_{\ell}^{U}\right\rangle^{k}=\sum_{i=1}^{n_{\text {cand }}^{k}}\left(w_{i} / \epsilon_{i}\right) P_{\ell}\left(\cos \theta_{\Lambda^{*}}^{i}\right) .
$$

Here the index $i$ runs over selected $J / \psi p K^{-}$candidates in the signal and sideband regions for the $k^{t h}$ bin of $m_{K p}\left(n_{\text {cand }}{ }^{k}\right.$ is their total number $), \epsilon_{i}=\epsilon\left(m_{K p}{ }^{i}, \cos \theta_{\Lambda^{*}}{ }^{i}, \Omega_{a}{ }^{i}\right)$ is the efficiency correction, and $w_{i}$ is the background subtraction weight.

Now that the moments are computed, under the hypothesis that the $\Lambda^{*}$ states indeed follow the $\ell_{\max }$ limit, they need to compared to the data to see if they explain it. The best comparison is achieved using the $m_{J / \psi p}$ distribution. After some mathematical gymnastics the $\mathcal{P}\left(m_{J / \psi p} \mid H_{0}\right)$ distribution is formed and compared to the directly obtained efficiency-corrected and background-subtracted $m_{J / \psi p}$ distribution in the data in Fig. 20.

While it is clear to the naked eye that the $H_{0}$ hypothesis is a bad fit to the data, mostly due the structure near $4450 \mathrm{MeV}$, a quantitative method of distinguishing between this and other models is needed. Consider the specific alternative hypothesis that includes the two pentaquark states, $H_{1}$. To evaluate $\mathcal{P}\left(\cos \theta_{\Lambda^{*}} \mid H_{1}, m_{K p}{ }^{k}\right)$, similar to the determination of $H_{0}$ in Eq. 19 , $\ell_{\max }$ is increased to allow for the effects of pentaquark states on the higher moments. The actual choice of the new $\ell_{\max }$ is a matter of judgement: a very large increase would describe the data better, but at the expense of a weakened test due to adding possible additional fluctuations. In this analysis the maximum value somewhat arbitrarily was chosen as 31 .

A good metric to use in order to test for the best hypothesis is the likelihood ratio

$$
\Delta(-2 \ln L)=\sum_{i=1}^{n} w_{i} \ln \frac{\mathcal{P}\left(m_{J / \psi p}{ }^{i} \mid H_{0}\right) / I_{H_{0}}}{\mathcal{P}\left(m_{J / \psi p}{ }^{i} \mid H_{1}\right) / I_{H_{1}}},
$$

where $n$ is the number of signal and background events, $w_{i}$ the background subtraction weight, and the $I_{H}$ 's are normalization factors.

It is necessary to ascertain the distribution of the test variable for the $H_{0}$ hypothesis, $\mathcal{F}_{t}\left(m_{J / \psi p}^{i} \mid H_{0}\right)$. This is done by simulating many pseudo-experiments, varying the measured parameters, but keeping

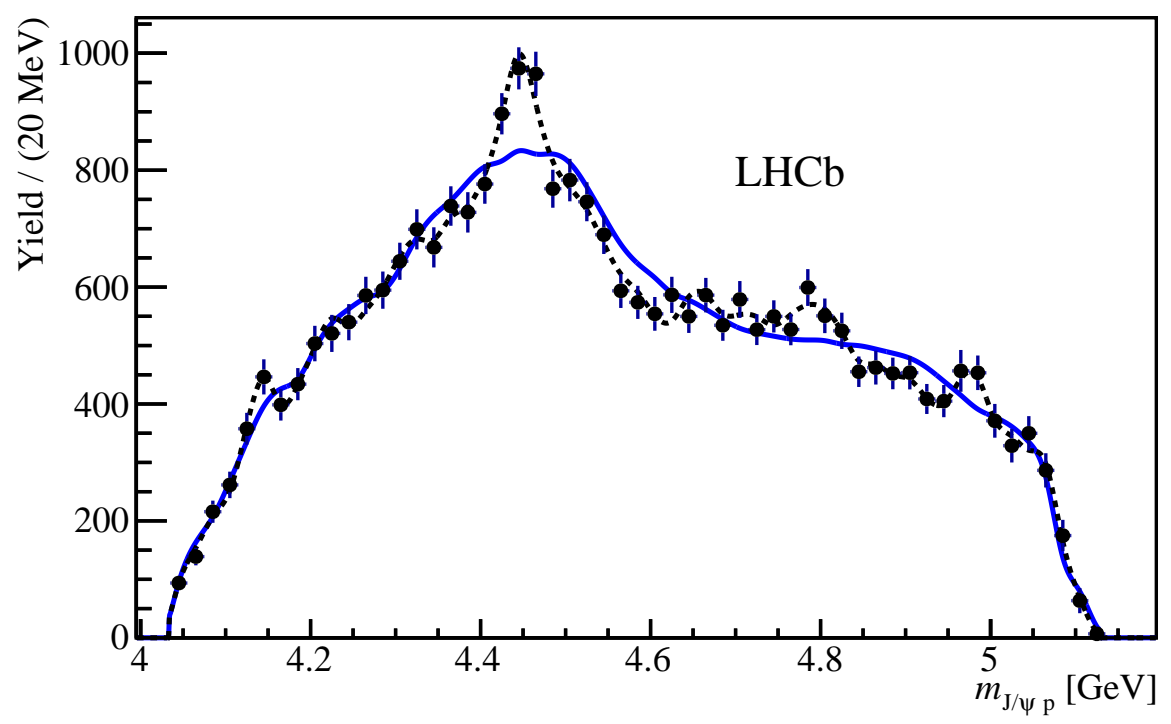

Figure 20: Efficiency-corrected and background-subtracted $m_{J / \psi p}$ distribution of the data (black points with error bars), with $\mathcal{P}\left(m_{J / \psi p} \mid H_{0}\right)$ (solid blue line) and $\mathcal{P}\left(m_{J / \psi p} \mid H_{1}\right)$ (dashed black line) superimposed (from $[11]$ ). 
the number of signal and background events fixed. Signal events are generated using the $\mathcal{P}\left(m_{J / \psi p}^{i} \mid H_{0}\right)$ function, while the background is generated using the parameters determined in the amplitude analysis in Ref. [103]. The distribution is shown in the Fig. 21, and in the inset in logarithmic scale.

It is interesting to see how adding $P_{c}^{+}$resonances into the simulation would change the liklihood function. When the $P_{c}(4380)^{+}$with $205 \mathrm{MeV}$ total width $(\Gamma)$ is added the liklihood distribution hardly changes from $\mathcal{F}_{t}\left(m_{J / \psi p}^{i} \mid H_{0}\right)$, (see Fig. 21) showing that this technique cannot detect its presence. Other simulations show that if its width were $102 \mathrm{MeV}$, or better yet $51 \mathrm{MeV}$ its presence would be clear. The prediction for both the $205 \mathrm{MeV} P_{c}(4380)^{+}$and the $\Gamma=39 \mathrm{MeV} P_{c}(4450)^{+}$are predicted to show a large separation and this is confirmed by the value of $\Delta(-2 \ln L)$ from the data, shown in the insert. The $p$-value obtained from Eq. 21 corresponds to 9 standard deviations and confirms the presence of the $P_{c}(4450)^{+}$.

\subsection{Impending studies using photoproduction of $J / \psi p$ resonances}

While LHCb has collected more data in 2015 and 2016, about doubling their sample of $\Lambda_{b}^{0}$ baryons, and will collect even more data in the future, from which more information on the $P_{c}^{+}$states will be extracted, it is very important to have confirmation of the two $P_{c}^{+}$states from another experiment. A very promising avenue was suggested soon after the pentaquark discovery was announced involving $J / \psi$ photoproduction via the reaction $\gamma p \rightarrow J / \psi p[113$ 115]. Since the photon has the same quantum numbers as the $J / \psi$ in the field of a particle it can transform temporarily into a $J / \psi$, or for that matter any vector meson. This process is called "vector dominance" [116]. The virtual $J / \psi$ then interacts with the proton. This process has been studied experimentally [12], and is thought to proceed via the diffractive diagram shown in Fig. 22(a) where the gluon exchanges are summarized as a "Pomeron." The resonant diagram is shown in Fig. 22(b).

The presence of resonant $P_{c}^{+}$states could be observed if $\mathcal{B}\left(P_{c}^{+} \rightarrow J / \psi p\right)$ is large enough. Fig. 23 shows the expected $\gamma p \rightarrow J / \psi p$ cross-section as a function of energy, assuming hat $\mathcal{B}\left(P_{c}^{+} \rightarrow J / \psi p\right)$ is $5 \%$. Measurements in the region where the $P_{c}$ states could be observed are scarce and easily could have missed any resonance structure because of acceptance and resolution issues. Experiments have been approved at Jefferson lab using a $12 \mathrm{GeV}$ photon beam $117-119$ and we eagerly await the results.

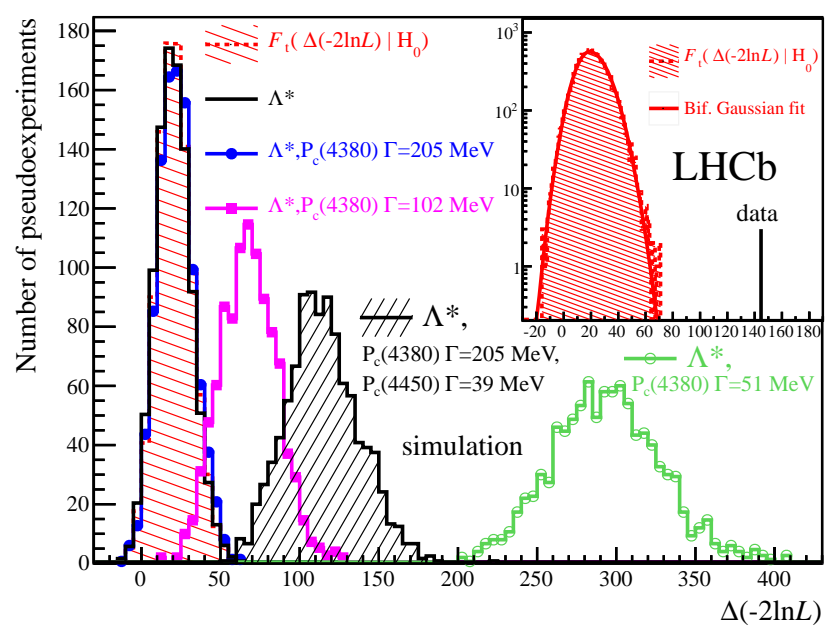

Figure 21: Distributions of $\Delta(-2 \ln L)$ in the model-independent pseudo-experiments corresponding to $H_{0}$ (red falling hatched) compared to the distributions for pseudo-experiments generated from various amplitude models and, in the inset, to the bifurcated Gaussian fit function (solid line) and the value obtained for the data (vertical bar) (from [111]). 

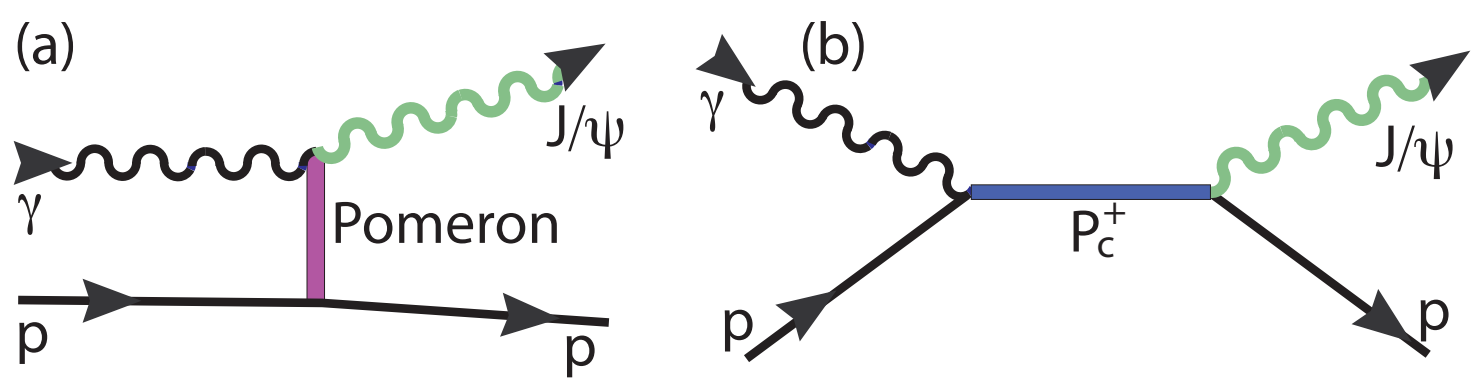

Figure 22: Feynman diagrams for $\gamma p \rightarrow J / \psi p$ via (a) the diffractive (Pomeron) process and (b) mediated by the $P_{c}^{+}$states.
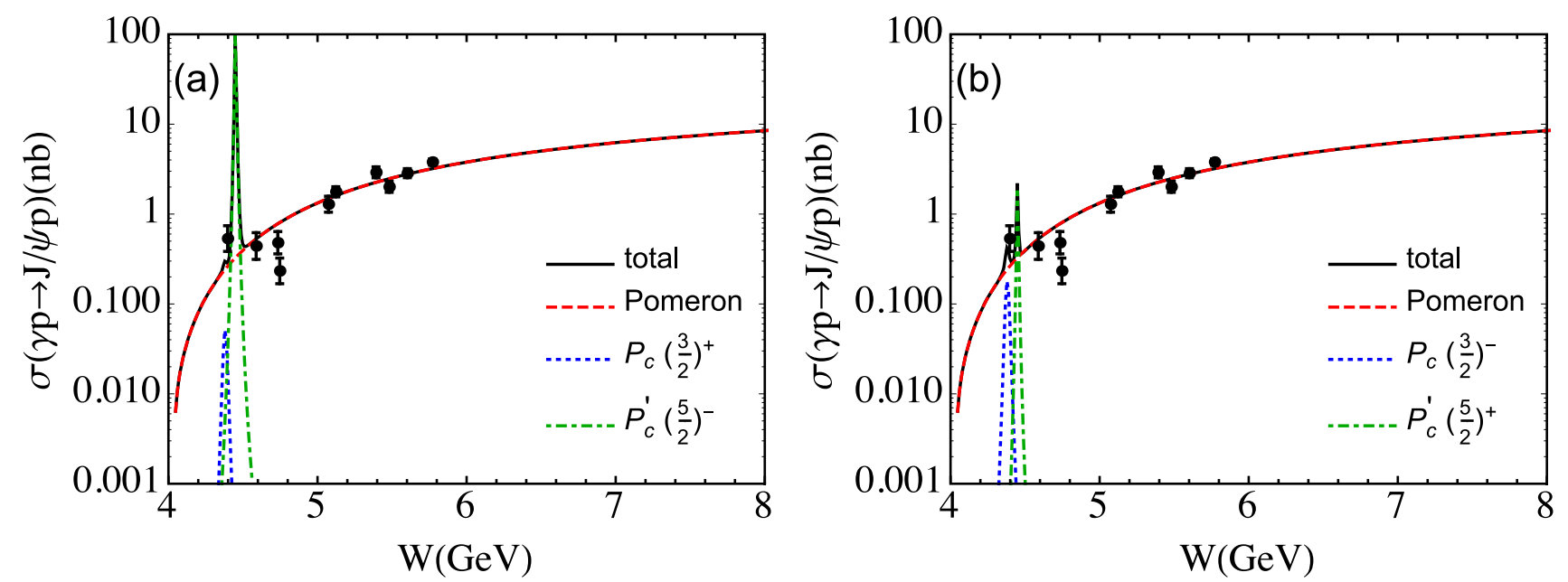

Figure 23: Measurements of the $\gamma p \rightarrow J / \psi p$ cross-section as a function of the total center-of-mass energy $\mathrm{W}$ for the diffractive (Pomeron) process and the two observed $P_{c}^{+}$states, under the assumption that $\mathcal{B}\left(P_{c}^{+} \rightarrow J / \psi p\right)$ is $5 \%$, for two different parity assignments as indicated (from 113 ).

\section{Experimental evidence for tetraquarks}

\subsection{The $X(3872)$ state}

Results of the E705 experiment from interactions of a $300 \mathrm{GeV} / \mathrm{c}$ momentum $\pi^{ \pm}$beam on a nuclear $L i$ target reported in 1993 indicated a state just above $3.8 \mathrm{GeV}$ in mass decaying into $J / \psi \pi^{+} \pi^{-}[120]$. The first observation was made by Belle [121] in a data set of $152 \times 10^{6} B \bar{B}$ events with a large statistical significance of $9.4 \sigma$ in the decay $B^{ \pm} \rightarrow K^{ \pm} X(3872)\left(\rightarrow J / \psi \pi^{+} \pi^{-}\right)$. Since an assignment of a charmonium state was a priori not possible, the state was called $X(3872)$. Soon afterwards it was confirmed by BABAR [3, 4], CDF II [5 7], D0 [8], LHCb [9] and CMS [10].

Two measurements serve as examples of two different production mechanisms. First of all, in $B$ mesons decays, Belle performed an analysis using the complete data set of $711 \mathrm{fb}^{-1}$ collected at the $\Upsilon(4 S)$ resonance [122]. The decays $B^{+} \rightarrow K^{+} X(3872)$ and $B^{0} \rightarrow K^{0}\left(\rightarrow \pi^{+} \pi^{-}\right) X(3872)$ were investigated. For the determination of the mass and the width (see below) of the $X(3872)$ in the mode $X(3872) \rightarrow J / \psi \pi^{+} \pi^{-}$, a 3-dimensional fit was performed using the three variables: beam constraint mass $M_{\mathrm{bc}}=\sqrt{\left(E_{\text {beam }}^{c m s}\right)^{2}-\left(p_{B}^{c m s}\right)^{2}}$ (with the energy in the center-of-mass system $E_{\text {beams }}^{c m s}$, and the momentum of the $B$ meson in the center-of-mass system $\left.p_{B}^{c m s}\right)$, the invariant mass $m\left(J / \psi \pi^{+} \pi^{-}\right)$and the energy difference $\Delta E=E_{B}^{c m s}-E_{\text {beam }}^{c m s}$ (with the energy of the $B$ meson in the center-of-mass system $E_{B}^{c m s}$ ). For the mass measurement, in a first step, the fit was performed for the reference channel $\psi^{\prime} \rightarrow J / \psi \pi^{+} \pi^{-}$, and the resolution parameters (i.e. the widths of a core Gaussian and a tail Gaussian) were then fixed for the fit of the $X(3872)$. Fig. 24 (top) shows the data and the fits for the $X(3872)$ in the projections 

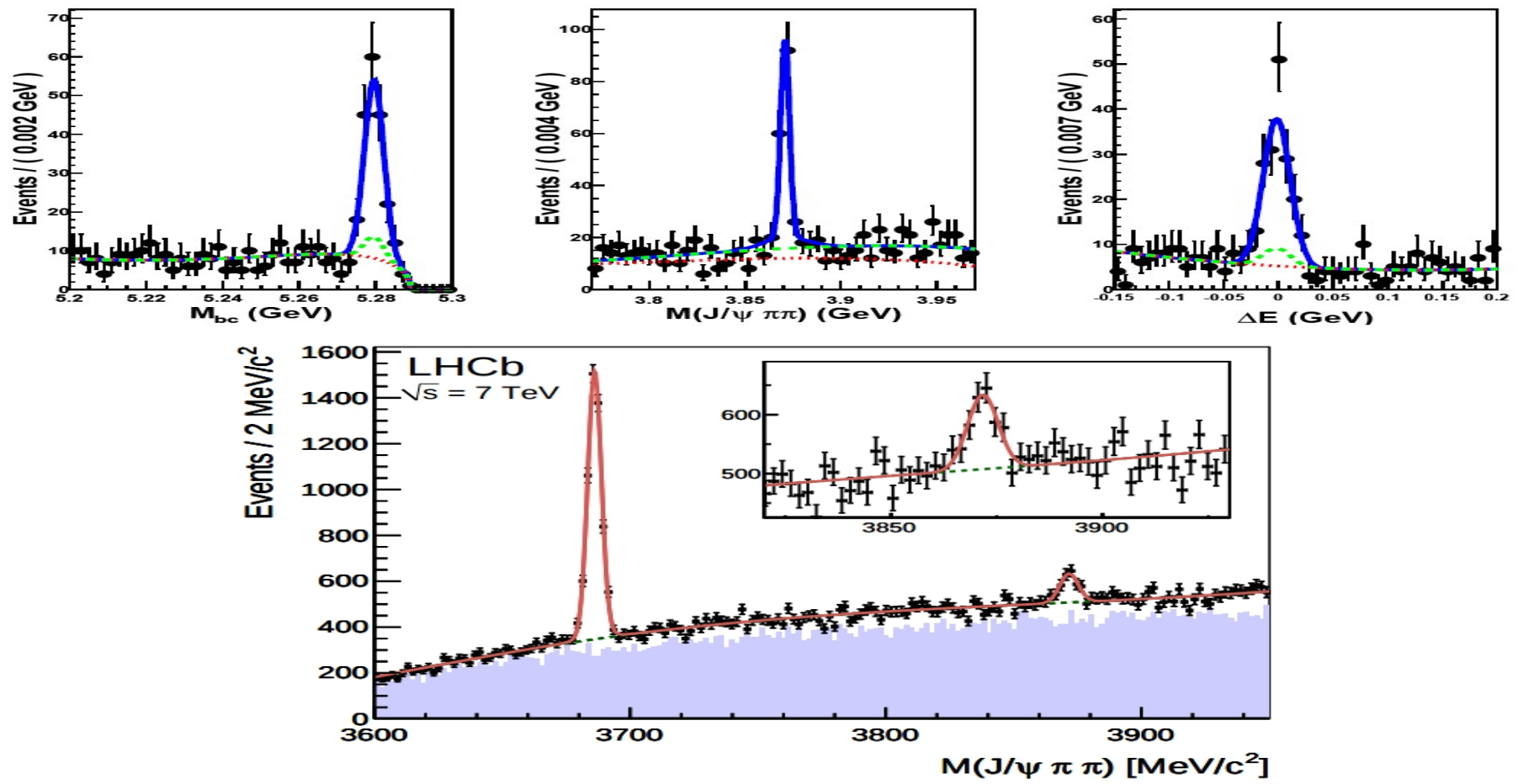

Figure 24: Top: Beam constraint mass $M_{\mathrm{bc}}$ (left), invariant mass $m\left(J / \psi \pi^{+} \pi^{-}\right)$(center) and $\Delta E$ (right) for $B^{+} \rightarrow K^{+} X(3872)\left(\rightarrow J / \psi \pi^{+} \pi^{-}\right)$from Belle [122]. The blue line indicates signal, and the dashed green line background. Bottom: Invariant mass $m\left(J / \psi \pi^{+} \pi^{-}\right)$in inclusive production in $p p$ collision at $\sqrt{s}=7 \mathrm{TeV}$ from LHCb $[9]$.

of the three variables as defined above. The yield is $151 \pm 15$ events for $B^{+}$decays and $21.0 \pm 5.7$ events for $B^{0}$ decays.

Secondly, LHCb observed the inclusive production of the $X(3872)$ in $p p$ collisions at $\sqrt{s}=7 \mathrm{TeV}$ with an integrated luminosity of $34.7 \mathrm{pb}^{1}[9]$. Fig. 24 (bottom) shows the invariant mass $m\left(J / \psi \pi^{+} \pi^{-}\right)$with a fitted signal yield of $565 \pm 62$ events for the $X(3872)$. In an updated analysis [123] using increased integrated luminosity of $1 \mathrm{fb}^{-1}$, but a tighter selection, a signal yield $313 \pm 26$ was reported.

\subsubsection{Mass of the $X(3872)$}

The world average mass of the $X(3872)$ is $3871.69 \pm 0.17 \mathrm{MeV}[12]$. As will be discussed below, the $X(3872)$ does not fit into potential model predictions, and is discussed in literature as a possible $S$-wave $D^{* 0} \bar{D}^{0}$ molecular state, in particular because its mass is close to the $D^{* 0} \bar{D}^{0}$ threshold. In this case, the binding energy $E_{b}$ is given by the mass difference $m(\mathrm{X})-m\left(D^{* 0}\right)-m\left(D^{0}\right)$, yielding $E_{b}=0.01 \pm 0.18 \mathrm{MeV}$ using present world average masses of the $X$, the $D^{0}$ and the $D^{* 0}[12$. For an $S$-wave near-threshold resonance with an assumed positive scattering length, $E_{b}$ is inverse proportional to the squared scattering length $a$ according to $E_{b}=\hbar^{2} / 2 \mu a^{2}[124-126$ using the reduced mass $\mu$. The average radius can be approximated in first order by $\langle r\rangle=a / \sqrt{2}$, which would lead to a very large value of $<r>\geq 31.7_{-24.5}^{+\infty} \mathrm{fm}$.

\subsubsection{Width of the $X(3872)$}

Belle used the above mentioned 3-dimensional fit to attempt to measure the width. The 3-dimensional fits are more sensitive to the natural width than the mass resolution provided by the detector because of the constraints which enter from additional kinematic parameters. In the first step, the fit was performed for the reference channel $\psi^{\prime} \rightarrow J / \psi \pi^{+} \pi^{-}$, and the resolution parameters (i.e. the widths of a 
core Gaussian and a tail Gaussian) were then fixed for the fit of the $X(3872)$. With this 3-dimensional fit, a new precise measurement of the width of the $X(3872)$ was performed. This method of determining the width was tested using the $\psi^{\prime}$ as reference providing a result of $\Gamma_{\psi^{\prime}}^{\text {measured }}=0.52 \pm 0.11 \mathrm{MeV}$ larger than the world average of $\Gamma_{\psi^{\prime}}^{P D G}=0.304 \pm 0.009 \mathrm{MeV}[12$, indicating a bias in the Belle measurement of $\Delta \Gamma=+0.23 \pm 0.11 \mathrm{MeV}$. The procedure for the determination of the upper limit on $\Gamma_{X(3872)}$ is: for a given fixed width $\Gamma$ the number of signal events and the number of peaking background events is kept floating in the 3-dimensional fit, and the likelihood is calculated. Then the $90 \%$ likelihood interval is determined by finding $w_{90 \%}$ for the integral $\int_{0}^{w_{90}} \Gamma d \Gamma=0.9$. This procedure gives $w_{90 \%}=0.95 \mathrm{MeV}$, to which the bias has to be added, so that the final result is $\Gamma_{X(3872)}<1.2 \mathrm{MeV}$ at $90 \%$ C.L. This upper limit is a factor of $\simeq 2$ smaller than the previous upper limit. Higher precision might be difficult to achieve using this method, however, the width of the $X(3872)$ can be measured by a resonance scan using a cooled anti-proton beam [127], [128]. The narrow width of the $X(3872)$ is quite remarkable, as it is about two orders of magnitude smaller than potential model predictions for the $\chi_{c 1}^{\prime}$, a predicted charmonium state with nearby mass and identical quantum numbers. One of the proposed explanations is isospin violation (see below).

\subsubsection{Decays of the $X(3872)$}

The $X(3872)$ is one of the few among the new charmonium-like states which has been observed in more than one decay channel, clearly proving that it is not a threshold effect. The decay channels are $X(3872) \rightarrow J / \psi \pi^{+} \pi^{-}$[121] [3, 4] [5 7] [8], $X(3872) \rightarrow J / \psi \gamma$ [129] [130], $X(3872) \rightarrow \psi^{\prime} \gamma$ [130] [131], $X(3872) \rightarrow J / \psi \pi^{+} \pi^{-} \pi^{0}$ 132], $X(3872) \rightarrow D^{0} \bar{D}^{0} \pi^{0}$ [133 [134], and $X(3872) \rightarrow D^{0} \bar{D}^{0} \gamma$ [134].

Decays of $\boldsymbol{X}(\mathbf{3 8 7 2})$ to $\boldsymbol{J} / \boldsymbol{\psi} \boldsymbol{\pi} \boldsymbol{\pi}$ The decay $X(3872)$ to $J / \psi \pi^{+} \pi^{-}$was the first observed decay mode of the $X(3872)\left[121\right.$. The $\pi^{+} \pi^{-}$invariant mass shows evidence that the decay actually proceeds through the sequential decay $X(3872) \rightarrow J / \psi \rho$ with $\rho \rightarrow \pi^{+} \pi^{-}$(see below). For the case of non-resonant $\pi^{+} \pi^{-}$, the isospin could be $I=0$ or $I=1$. However, for the case of an intermediate $\rho$ the isospin is fixed to $I=1$. Therefore the decay $X(3872) \rightarrow J / \psi \rho$ violates strong isospin conservation. There are only two additional isospin violating transitions known in the charmonium system [12], namely $\psi^{\prime} \rightarrow J / \psi \pi^{0}$ $\left(\mathcal{B}=1.3 \pm 0.1 \times 10^{-3}[12], \mathcal{B}=1.26 \pm 0.02 \pm 0.03 \times 10^{-3}[135]\right)$ and $\psi^{\prime} \rightarrow h_{c} \pi^{0}\left(\mathcal{B}=8.4 \pm 1.6 \times 10^{-4}[12]\right)$. For the $X(3872)$ the branching fraction of isospin violating transition is (among the known decays) $>2.6 \%$ [12] and thus seems to be largely enhanced. There are several possible mechanisms for the isospin violation:

- The $u / d$ quark mass difference in strong interaction can induce isospin violation. Such a difference is immediately provided here, as the $X(3872)$ can only decay to $D^{0 *} \bar{D}^{0}$ (containing $u$-type quarks), but not into $D^{+*} D^{-}$(containing $d$-type quarks), for which the threshold is $\simeq 8 \mathrm{MeV}$ higher.

- The $u / d$ quark charge difference in electromagnetic interactions (EM) can induce isospin violation. Isospin should only be conserved in strong interaction, but not in EM interaction. Thus, one of the possible explanation is that the decay $X(3872) \rightarrow J / \psi \rho\left(\rightarrow \pi^{+} \pi^{-}\right)$is proceeding via EM interaction, i.e., the $\rho$ is created not by gluons but by a virtual photon. The branching ratio would be then comparable to radiative decays (see below).

- As the $\rho$ has a large width of $149.1 \pm 0.8 \mathrm{MeV}[12]$, mixing of $\rho$ and $\omega$ can induce isospin violation 136 .

- Rescattering such as $X(3872) \rightarrow D^{0 *} \bar{D}^{0} \rightarrow D^{+*} D^{-} \rightarrow J / \psi \rho$ or $X(3872) \rightarrow D^{0} \bar{D}^{0} \gamma \rightarrow D^{+} D^{-} \gamma \rightarrow J / \psi \rho$ may induce isospin violation $[137-142]$. Although the threshold for the charged $D$ mesons is $\simeq 8 \mathrm{MeV}$ higher than the mass of the $X(3872)$, they may contribute in hadronic loops as a virtual state. 

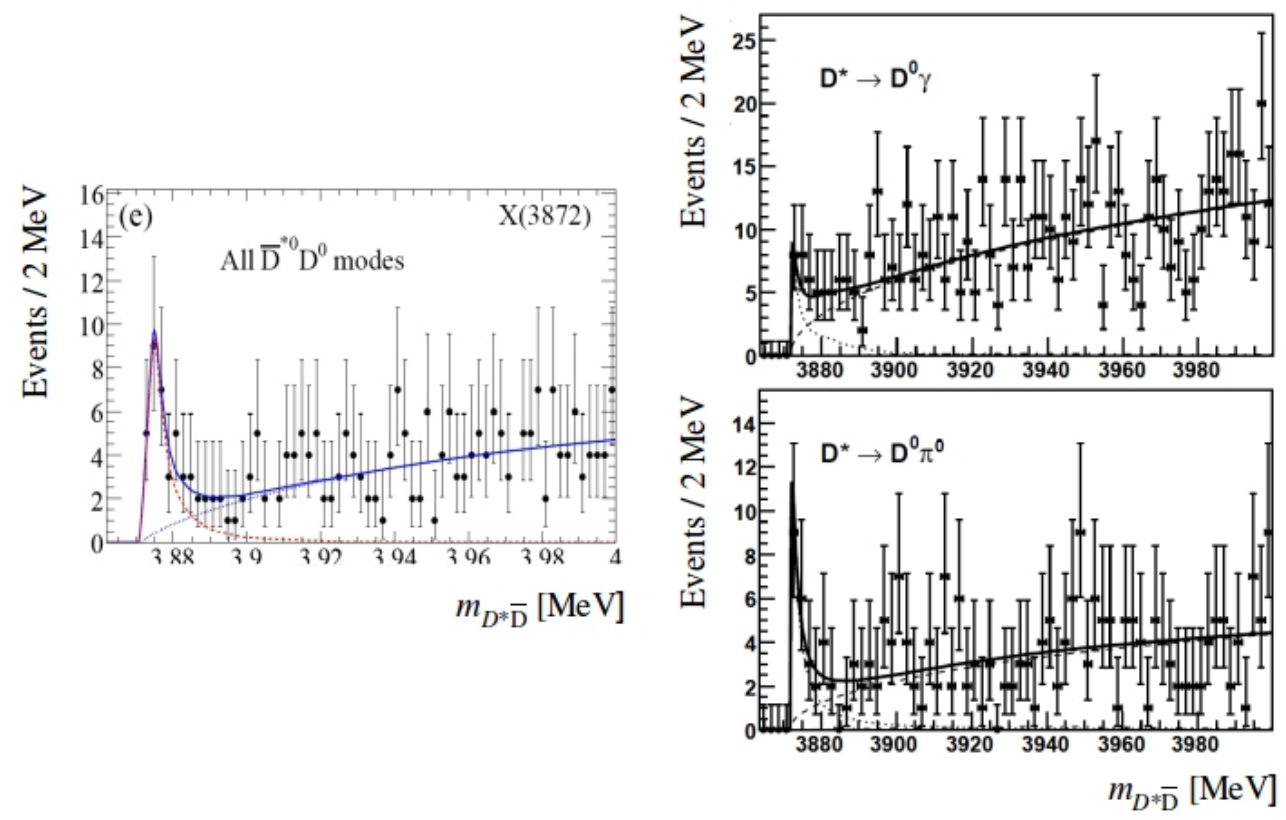

Figure 25: $D^{0 *} \bar{D}^{0}$ invariant mass for the decay $X(3872) \rightarrow D^{0 *} \bar{D}^{0}$ from $B A B A R$ 143 (left, for both $D^{* 0} \rightarrow D^{0} \gamma$ and $D^{* 0} \rightarrow D^{0} \pi^{0}$ ) and from Belle [133] [134 (right top for $D^{* 0} \rightarrow D^{0} \gamma$ and right bottom for $\left.D^{* 0} \rightarrow D^{0} \pi^{0}\right)$.

Decays of $\boldsymbol{X}(\mathbf{3 8 7 2})$ to $\boldsymbol{D}^{(*)} \overline{\boldsymbol{D}}^{(*)} \quad$ As the mass of the $X(3872)$ is very close to the $D^{0} \bar{D}^{0 *}$ threshold, the investigation of open charm decays is very important. The decay into $D^{0} D^{0 *}$ is a strong decay and among the so far observed decays it represents the dominant one, i.e. the branching fraction is a factor $\simeq 9$ higher than for the decay into $J / \psi \pi^{+} \pi^{-}$. In this decay channel, BABAR measured surprisingly a high mass of the $X(3872)$ as $m=3875.1_{-0.5}^{+0.7}$ (stat.) \pm 0.5 (syst.) $\mathrm{MeV} 143$ (see Fig. 25, left), suggesting that there might be two different states, namely $X(3872)$ and $X(3876)$, which would fit to a tetraquark hypothesis $[144$ for two states $[c \bar{c} u \bar{u}]$ and $[c \bar{c} d \bar{d}]$. On the other hand, Belle measured the mass in the same decay channel as $m=3872.9_{-0.4}^{+0.6}$ (stat.) ${ }_{-0.5}^{+0.4}$ (syst.) $\mathrm{MeV}[134]$ (see Fig. 25, right) which is consistent with the world average [12]. A possible explanation of the discrepancy is the difficulty of performing fits to signals close to threshold. In fact, the two experiments used two very different approaches:

- BABAR used a 1-dimensional binned maximum likelihood fit 143 with the $D^{*} D$ invariant mass as the only variable, where the signal probability density function (p.d.f.) was extracted from MC simulations assuming a $S=1$ resonance produced with $L=0$. An exponential function $a(m-$ $\left.m_{0}\right)^{b} \times \exp \left(m-m_{0}\right)$ with a threshold mass $m_{0}$ and parameters $a, b, c$ was used for the background parametrization.

- Belle used an 2-dimensional unbinned maximum likelihood fit [134] to the beam constrained mass, with a Gaussian signal function and an Argus function 145 for the background, and the $D^{*} D$ invariant mass using a Breit-Wigner signal function and $a \sqrt{m-m_{0}}$ for the background parametrization.

Following a suggestion from [146] the fit in the Belle case was checked with a Flatté p.d.f. instead of a Breit-Wigner p.d.f., in particular to take into account the contribution of $D^{+*} D^{-}$to the tail shape. Results were consistent.

An important question is if the decay proceeds resonant in the $D^{*}$ (i.e. $X(3872) \rightarrow D^{0} \bar{D}^{0 *}$ ) or nonresonant (i.e. $\left.X(3872) \rightarrow D^{0} \bar{D}^{0} \pi^{0}\right)$. As the $D^{* 0}\left(D^{0}\right)$ carries $J=1(J=0)$, the first case would correspond to $L=0$, the latter case to $L=1$. In the analyses, a mass cut on the $D^{*}$ can distinguish between the 
resonant and non-resonant case. The product branching fractions for the sum of the resonant and nonresonant case is $\left(1.22 \pm 0.31_{-0.30}^{+0.23}\right) \times 10^{-4}\left[133\right.$, for the resonant case alone $(0.80 \pm 0.20 \pm 0.10) \times 10^{-4}[134]$ and thus corresponding to $\simeq 65 \%$. Note that the decay of $D^{0 *} \rightarrow D^{0} \gamma$ (Fig. 25, right top) only contributes to the resonant case. So far no evidence for non-resonant $X(3872) \rightarrow D^{0} \vec{D}^{0} \gamma$ has been reported. The charged decay $X(3872) \rightarrow D^{+} D^{*-}$ was not observed, as the mass of the $X(3872)$ is $8.1 \mathrm{MeV}$ below the threshold and consequently is kinematically forbidden.

Radiative Decays of the $\boldsymbol{X}(\mathbf{3 8 7 2}) \quad$ The decay $X(3872) \rightarrow J / \psi \gamma$ represents a decay into two eigenstates of C-parity. Therefore its observation implies that the charge conjugation of the $X(3872)$ must be $\mathrm{C}=+1$. The observation of this decay was reported by Belle with a data set of $256 \mathrm{fb}^{-1}$, a yield of $13.6 \pm 4.4$ events and a statistical significance of $4.0 \sigma[129]$. The combined branching ratio was measured to $\left.\mathcal{B}\left(B^{ \pm} \rightarrow X K^{ \pm}, X \rightarrow \gamma J / \psi\right)=(1.8 \pm 0.6 \pm 0.1) \times 10^{-6}\right)$, i.e. the branching fraction of the rare decay $X(3872) \rightarrow J / \psi \gamma$ is a factor $\simeq 6$ smaller than the one for $X(3872) \rightarrow J / \psi \pi^{+} \pi^{-}$. BABAR confirmed the observation with a data set of $260 \mathrm{fb}^{-1}$, a yield of $19.4 \pm 5.7$ events and a statistical significance of $3.4 \sigma$ [130 (see Fig. 26, bottom). The combined branching ratio was measured to $\mathcal{B}\left(B^{ \pm} \rightarrow X K^{ \pm}\right.$, $\left.X \rightarrow \gamma J / \psi)=(3.4 \pm 1.0 \pm 0.3) \times 10^{-6}\right)$, i.e. about a factor of two higher than Belle.

BABAR found evidence for the decay $X(3872) \rightarrow \psi^{\prime} \gamma[3]$ with $424 \mathrm{fb}^{-1}$ and $25.4 \pm 7.4$ signal events. The ratio of the branching fractions $R_{\psi \gamma}=\mathcal{B}(\mathrm{X}(3872) \rightarrow \psi(2 \mathrm{~S}) \gamma) / \mathcal{B}(\mathrm{X}(3872) \rightarrow J / \psi \gamma)$ was measured as $R_{\psi \gamma}=3.4 \pm 1.4$. indicating the surprising fact that the transition of the $X(3872)$ to the $n=2$ state is significantly stronger than the transition to the $n=1$ state. In the case of $X(3872) \rightarrow J / \psi \gamma$ the photon energy is $E_{\gamma}=775 \mathrm{MeV}$, and thus due to vector meson dominance $\rho$ and $\omega$ can contribute to the amplitudes. However, in the case of $X(3872) \rightarrow \psi^{\prime} \gamma$ with the smaller $E_{\gamma}=186 \mathrm{MeV}$ the transition can only proceed through light quark annihilation with an expected small amplitude.
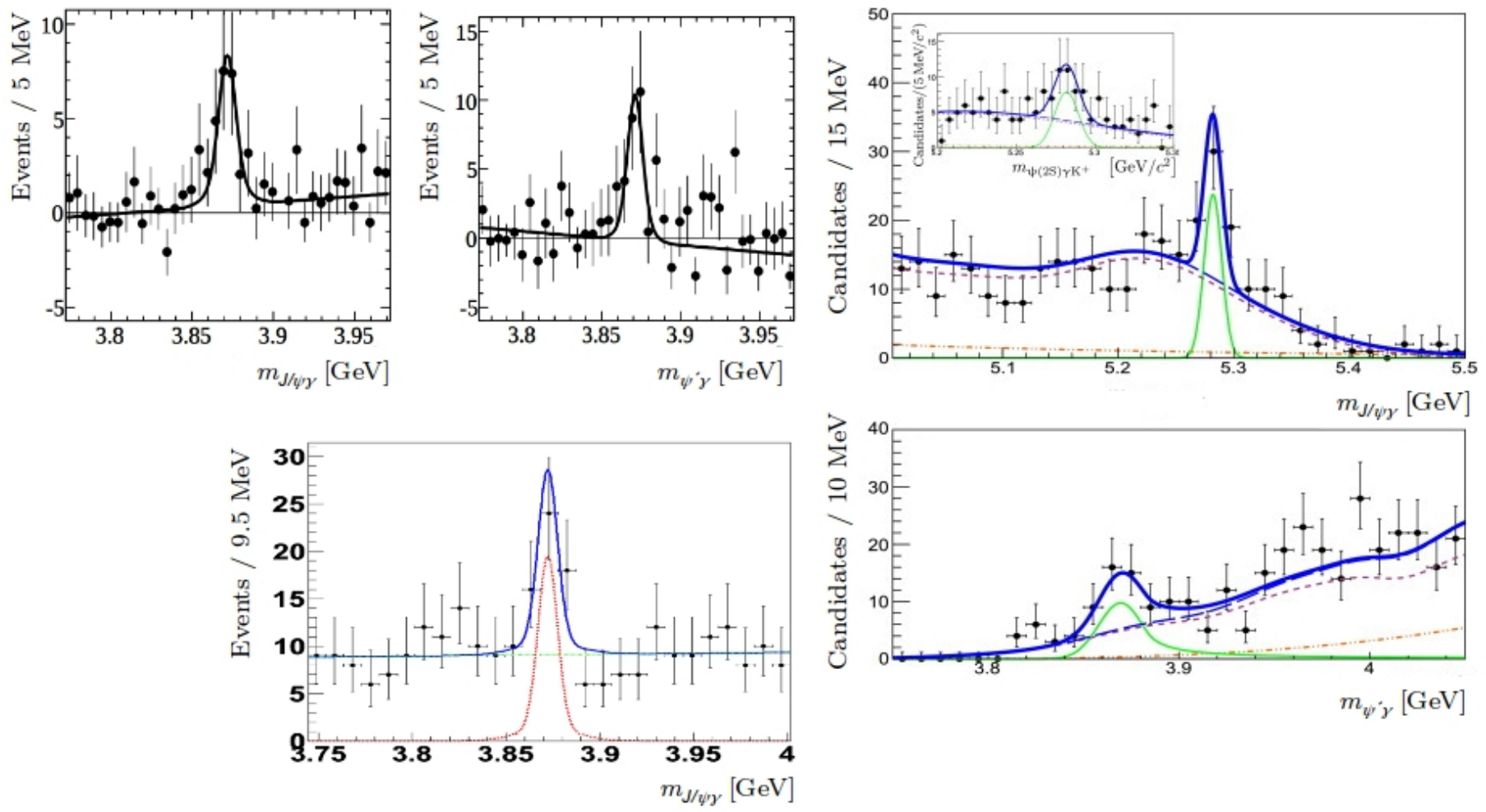

Figure 26: $J / \psi \gamma$ and $\psi^{\prime} \gamma$ invariant mass for the decays $X(3872) \rightarrow J / \psi \gamma$ and $X(3872) \rightarrow \psi^{\prime} \gamma$ from $B A B A R$ [130] (top left, top center), from Belle [147] (bottom left, only $J / \psi \gamma$ observed) and from LHCb [131] (top right, bottom right). 
The ratio $R_{\psi \gamma}$ is predicted to be in the range $(3-4) \times 10^{-3}$ for a $D \bar{D}^{*}$ molecule 148 150], $1.2-$ 15 for a pure charmonium state $[26,151,156]$ and $0.5-5$ for a molecule-charmonium mixture [154, 157. An updated measurement by Belle of both radiative channels was based upon a data set of $711 \mathrm{fb}^{-1}$ [147]. The background was studied in MC simulations and revealed peaking behavior in some background components close to the signal region. The signal $X(3872) \rightarrow J / \psi \gamma$ was clearly re-established (see Fig. 26, top) with $30.0_{-7.4}^{+8.2}$ signal events $\left(4.9 \sigma\right.$ significance) for $B^{+} \rightarrow K^{+} X(3872)$ and $5.7_{-2.8}^{+3.5}$ signal events $\left(2.4 \sigma\right.$ significance) for $B^{0} \rightarrow K^{0} X(3872)$. For $X(3872) \rightarrow \psi^{\prime} \gamma$, the shape of the $\psi^{\prime} K^{*}$ and $\psi^{\prime} K$ background, and in particular the peaking structures, was modeled as a sum of bifurcated Gaussians using a large MC sample. The signal yields were determined as $5.0_{-11.0}^{+11.9}$ signal events $(0.4 \sigma$ significance $)$ for $B^{+} \rightarrow K^{+} X(3872)$ and $1.5_{-3.9}^{+4.8}$ signal events $\left(0.2 \sigma\right.$ significance) for $B^{0} \rightarrow K^{0} X(3872)$. With an upper limit of $R_{\psi \gamma}<2.1$ (90\% CL), Belle was not able to confirm the large $R_{\psi \gamma}$ value by BABAR .

Finally, with an integrated luminosity of $3 \mathrm{fb}^{-1}$, LHCb confirmed the evidence of $X(3872) \rightarrow \psi^{\prime} \gamma$ with a higher signal yield of $36.4 \pm 9.0$ [131], corresponding to a significance of $4.4 \sigma$. A large ratio of $R_{\psi \gamma}=2.46 \pm 0.64 \pm 0.29$ was measured, supporting the charmonium interpretation and the interpretation of a mixture of a molecule and a charmonium state, but disfavoring a pure molecule.

Decays of $\boldsymbol{X}(\mathbf{3 8 7 2})$ into $\boldsymbol{J} / \boldsymbol{\psi} \pi \boldsymbol{\pi}$ As one of the observed proposed explanations for the isospin violation (see above) is $\rho-\omega$ mixing [136], the search for the decay $X(3872) \rightarrow J / \psi \omega\left(\rightarrow \pi^{+} \pi^{-} \pi^{0}\right)$ is of importance. The difficulty here is the nearby $Y(3940)$ state, which is also known to decay into the same final state. Belle observed a signal for $X(3872) \rightarrow J / \psi \omega\left(\rightarrow \pi^{+} \pi^{-} \pi^{0}\right)$, based upon a data set of $256 \mathrm{fb}^{-1}$ [132]. A cut on the $\omega$ meson in the 3 -pion mass from 0.750 to 0.775 was used in the analysis. The observed yield was $12.4 \pm 4.1$ events, corresponding to a significance of $4.3 \sigma$. The measured efficiency corrected ratio of $X(3872) \rightarrow J / \psi \pi^{+} \pi^{-} \pi^{0} / X(3872) \rightarrow J / \psi \pi^{+} \pi^{-}=1.0 \pm 0.4$ (stat.) \pm 0.3 (syst.) indicates isospin violation in a decay of the $X(3872)$, as the additional $\pi^{0}$ carries $I=1$.

In an analysis by BABAR [158], using a three-pion mass from 0.7695 to 0.7965 as a cut for the $\omega$, no evidence for $X(3872) \rightarrow J / \psi \omega\left(\rightarrow \pi^{+} \pi^{-} \pi^{0}\right)$ was found. In a re-analysis with $426 \mathrm{fb}^{-1}$ by $B A B A R$ 159 the lower boundary of the kinematical search window for the three-pion mass was extended from $0.5 \mathrm{GeV}$ to $0.9 \mathrm{GeV}$. Here, the Belle signal was confirmed with a significance of $4.0 \sigma$. Also the large isospin violation is suggested by the measurement of the ratio $X(3872) \rightarrow J / \psi \pi^{+} \pi^{-} \pi^{0} / X(3872) \rightarrow J / \psi \pi^{+} \pi^{-}$as $0.7 \pm 0.3$ (stat.) and $1.7 \pm 1.3$ (stat.) for $B^{+}$and $B^{0}$ decays, respectively.

For the three decays with the largest branching fraction, numbers are given in Table6. The product branching fraction for the $J / \psi \pi^{+} \pi^{-}$final state was derived from 129 by using published branching fractions for the $\psi(2 S)$ in 12 . The relative rates for $J / \psi \pi^{+} \pi^{-}: D^{* 0} \bar{D}^{0}: J / \psi \gamma$ are $1: 0.18: 0.025$.

Table 6: Branching fractions of decays of the $X(3872)$.

\begin{tabular}{lcr|r}
\hline Product branching fraction & & \\
\hline $\mathcal{B}(B \rightarrow K X) \times \mathcal{B}\left(X(3872) \rightarrow J / \psi \pi^{+} \pi^{-}\right)$ & $(1.31 \pm 0.24 \pm 0.13) \times 10^{-5}$ & 121 \\
& $(1.28 \pm 0.41) \times 10^{-5}$ & 1 \\
\hline $\mathcal{B}(B \rightarrow K X) \times \mathcal{B}\left(X(3872) \rightarrow D^{* 0} \bar{D}^{0}\right)$ & $(0.73 \pm 0.17 \pm 0.08) \times 10^{-4}$ & 133 & 134 \\
\hline $\mathcal{B}(B \rightarrow K X) \times \mathcal{B}(X(3872) \rightarrow J / \psi \gamma)$ & $(1.41 \pm 0.30 \pm 0.22) \times 10^{-4}$ & 143 \\
\hline
\end{tabular}

\subsubsection{Production of the $X(3872)$}

The $X(3872)$ was discovered in three different $B$ meson decays $B^{0} \rightarrow K^{0}\left(\rightarrow \pi^{+} \pi^{-}\right) X(3872), B^{ \pm} \rightarrow$ $K^{ \pm} X(3872)$, and $B^{0} \rightarrow K^{0 *}\left(\rightarrow K^{+} \pi^{-}\right) X(3872)$. It has also been observed as an inclusive signal in 
direct $p \bar{p}$ collisions at CDF II [5 7], CMS [10], D0 [8] and LHCb [9] (see Fig. 24). However, so far, there was no indication of direct $X(3872)$ production in $e^{+} e^{-}$collisions, $\gamma \gamma$ collisions or production in initial state radiation. For the observation in $B$ meson decays, it is important to note that the $K^{0}, K^{ \pm}$have $J=0$, while the $K^{*}$ has $J=1$. Thus, the $X(3872)$ has been observed in $B$ meson decays both with a pseudoscalar or a vector particle accompanied. No evidence of the $X(3872)$ was found in $\gamma \gamma \rightarrow J / \psi \omega$ [160] [161] as expected, because it should not be observable for $J=1$ due to the Landau-Young theorem.

Production of $\boldsymbol{X}(\mathbf{3 8 7 2})$ in $\boldsymbol{B} \rightarrow \boldsymbol{K} \boldsymbol{\pi} \boldsymbol{X} \mathbf{( 3 8 7 2 )}$ All the above analyses of $X(3872)$ were performed in the decays $B \rightarrow K X(3872)$. However, it was also observed in $B \rightarrow K \pi X(3872)$ with an additional $\pi^{\mp}$. $B \rightarrow K \pi X(3872)$ can contain resonant $\left(K^{*}\right)$ and non-resonant amplitudes in the $K^{ \pm} \pi^{\mp}$ system, while $B \rightarrow K X(3872)$ is by definition only resonant. The non-resonant part was clearly observed and a product branching fraction of $\mathcal{B}\left(B^{0} \rightarrow X\left[K^{ \pm} \pi_{\text {non-resonant }}^{\mp}\right) \times \mathcal{B}\left(X \rightarrow J / \psi \pi^{+} \pi^{-}\right)=\left(8.1 \pm 2.0_{-1.4}^{+1.1}\right) \times 10^{-6}\right.$ was measured [162], corresponding to $N \simeq 90$ observed $X(3872)$ events. This is surprisingly as large as $\mathcal{B}\left(B^{0} \rightarrow \mathrm{XK}\right) \times \mathcal{B}\left(X \rightarrow J / \psi \pi^{+} \pi^{-}\right)=(8.10 \pm 0.92 \pm 0.66) \times 10^{-6}\left[162\right.$ and $(8.4 \pm 1.5 \pm 0.7) \times 10^{-6}[3,4]$, although the phase space is smaller. Another surprise is that the resonant part is very small, and only an upper limit $<3.4 \times 10^{-6}[162]$ could be given. This behavior is very different from other charmonium channels, e.g. $B \rightarrow K \pi \psi^{\prime}$ [162], $B \rightarrow K \pi J / \psi$ [163], or $B \rightarrow K \pi \chi_{c 1}^{\prime}$ [164]: in all cases the resonant $K^{*}(892)$ and $K^{*}(1430)$ are dominating these decays almost completely. This observation might support the indication that the $X(3872)$ does not represent conventional charmonium.

Production of $\boldsymbol{X}(\mathbf{3 8 7 2})$ in charged and neutral $\boldsymbol{B}$ decays Production of the $X(3872)$ in $B^{+}$and $B^{0}$ decays can be quite different from each other. According to 165 there are two different Feynman graphs for $B \rightarrow K D^{*} \bar{D}$ :

- In case of external $W$ emission the process is color enhanced, as the color is decoupled from the $B \rightarrow K$ transition. This process is possible for any of the transistions $B^{0} \rightarrow K^{+}, B^{0} \rightarrow K^{0}, B^{+} \rightarrow K^{+}$, and $B^{+} \rightarrow K^{0}$.

- In case of internal $W$ emission the color is locked by the spectator quark, which is in the same loop with the $\bar{D}$ and the $D^{*}$. This process is only possible for $B^{+} \rightarrow K^{+}$and $B^{0} \rightarrow K^{0}$, i.e. a change of the charge for the $B \rightarrow K$ transition is not possible. The charge sign flips by the $W$ boson, and then flips back again, when the loop is closed. This process is color suppressed.

In a factorization ansatz $124 \sqrt{126}$, the total amplitude for $B^{+} \rightarrow K^{+}$has three contributions: (1) $B^{+} \rightarrow \bar{D}^{* 0} V, V \rightarrow\left[D^{0} K^{+}\right]$, (2) $B^{+} \rightarrow \bar{D}^{0} V, V \rightarrow\left[D^{* 0} K^{+}\right]$, and (3) $B^{+} \rightarrow K^{+} V, V \rightarrow\left[\bar{D}^{* 0} D^{0}\right]$. All three involve the Cabibbo-allowed $\bar{b} \rightarrow \bar{c} W^{+}$, followed by $W^{+} \rightarrow c \bar{s}$, and a $q \bar{q}$ vacuum excitation, with (1) and (2) color-connected and (3) Fierz transformed. In contrast, the total amplitude for $B^{0} \rightarrow K^{0}$ has only one amplitude $B^{0} \rightarrow K^{0} V$ with $V \rightarrow\left[\bar{D}^{* 0} D^{0}\right]$. According to a detailed calculation [124 126], the ratio $\Gamma\left(B^{0} \rightarrow K^{0} X\right) / \Gamma\left(B^{+} \rightarrow K^{+} X\right)$ should be $\simeq 1$ for a charm meson molecular state. The most recent measurements of $0.41 \pm 0.24 \pm 0.05$ by BABAR [3,4] and $0.50 \pm 0.14 \pm 0.04$ by Belle [122] disfavour the molecule interpretation.

\section{Quantum numbers of the $X(3872)$}

Quantum Numbers from the $\mathbf{2} \boldsymbol{\pi}$ and $\mathbf{3} \boldsymbol{\pi}$ invariant mass distributions Early analyses [166] [167] tried to employ the shape of the $\pi^{+} \pi^{-}$mass distribution in the decay $X(3872) \rightarrow J / \psi \pi^{+} \pi^{-}$. If the relative angular momentum is fixed, then conclusions about the quantum numbers of the $X(3872)$ can be drawn. For $S$-wave the spectrum should show a dependance $\sim q^{*}(J / \psi)$ for $P$-wave $\sim q^{*}(J / \psi)^{2}$, where 
$q^{*}(J / \psi)$ denotes the momentum of the $J / \psi$ in the rest frame of the $X(3872)$. If the $X(3872)$ decays into two $J^{P}=1^{-}$particles (i.e. the $J / \psi$ and the $\rho$ ) with an $S$-wave, this implies that $J^{P}=0^{-}, 1^{+}, 2^{-}$, ... However, it was proven later by Belle [122], that $\rho-\omega$ interference has an significant impact on the shape, and $S$-wave and $P$-wave fits turned out to be both compatible with the observed distribution. In the analysis of the $X(3872) \rightarrow 3 \pi$ by BABAR [159], the shape of the $3 \pi$ mass distribution was investigated in order to constrain the quantum number of the $X(3872)$. It was found that, different from the case of the $2 \pi$ mass, the shape of the $3 \pi$ mass distribution seems to indicate that $P$-wave is preferred. Surprisingly, this implies $J^{P C}=2^{-+}$for the $X(3872)$, which was later proven to be incorrect by other analyses.

Quantum numbers from the decay into $J / \psi \gamma$ As mentioned above, the branching fraction of the the radiative decay $X(3872) \rightarrow J / \psi \gamma$ is about one order of magnitude smaller than $X(3872) \rightarrow J / \psi \pi^{+} \pi^{-}$ and about two orders of magnitude smaller than $X(3872)$ decaying into open charm. Although rare, this decay channel is very important, as its observation implies the decay into two particles, which are $\mathrm{C}=-1$, as they are identical with their anti-particles, and clearly establishes a $C=+1$ charge parity assignment to the $X(3872)$. Consequently, the $C=+1$ assignment importantly implies that the decays $X(3872) \rightarrow J / \psi \pi^{0} \pi^{0}, J / \psi \pi^{0}, J / \psi \eta, \chi_{c} \gamma$ or $\eta_{c} \gamma$ are forbidden. None of them has been observed.

The $S$-wave assignment from the $\pi^{+} \pi^{-}$invariant mass and the $C=+1$ assignment are consistent. As for mesons the total wave function must be symmetric, isospin $I=1$ and $J^{P}=1^{-}$for the case of the $\rho$ in combination of an $S$-wave does require $C=+1$. In addition, the search for a partner of the $X(3872)$ with negative $C$-parity in the decay $X(3872) \rightarrow J / \psi \gamma \gamma$ was negative [168] with a product branching fraction of $\mathcal{B}<1.9 \times 10^{-4}$.

Quantum numbers from angular analysis In order to apply further constraints on the quantum number assignment of the $X(3872)$, angular analyses have been performed. Initial studies by Belle [166] were based upon two assumptions:

- The decay is assumed to be $X(3872) \rightarrow J / \psi \rho$, rather than $X(3872) \rightarrow J / \psi \pi^{+} \pi^{-}$, i.e., a two-particle decay and not a three-particle decay.

- The polarisation of the $J / \psi$ is orthogonal to the axis of its decays to $e^{+} e^{-}$in its rest frame.

All the amplitudes are calculated in the $J / \psi$ rest frame. For the definition of the coordinate systems see [169 and [170]. $J^{P C}=0^{-+}$and $J^{P C}=0^{++}$were disfavoured by this analysis. The test for $J^{P C}=1^{+-}$ was disfavoured by an analysis based upon the $J / \psi$ helicity angle, i.e. the angle between the $J / \psi$ and the $B$ meson in the $X(3872)$ rest frame.

In an updated analysis by Belle [122 a test with the full data set was performed to distinguish in particular these two assignments $\left(J^{P C}=1^{++}\right.$or $\left.J^{P C}=2^{-+}\right)$using an angular analysis. For this purpose, it was assumed that the decay $X(3872) \rightarrow J / \psi \pi^{+} \pi^{-}$proceeds via $X(3872) \rightarrow J / \psi \rho\left(\rightarrow \pi^{+} \pi^{-}\right)$in the kinematic limit, i.e. both particles are at rest in the $X(3872)$ rest frame. Due to $m_{X(3872)} \simeq m_{\rho}+m_{J / \psi}$ this is a valid assumption and it also implies that any higher partial waves can be neglected. For $J^{P C}=1^{++}$, there is only one amplitude with $L=0$ and $S=1$, where $L$ is the total orbital angular momentum between the particles, and $S$ the total spin constructed from the $\rho$ and the $J / \psi$. For $J^{P C}=2^{-+}$, there are two amplitudes with $L=1$ and $S=1$ or $S=2$. These two amplitudes can be mixed using a parameter $\alpha$, which is a complex number. The angular reference frame follows the definition in [169]. The angle $\theta_{X}$ is chosen as the angle between the $J / \psi$ and the kaon direction in the $X(3872)$ rest frame. The angular distributions $d \Gamma / d \cos \left(\theta_{X}\right)$ for the different quantum numbers are: constant for $J^{P C}=1^{++}$, $\sin ^{2}\left(\theta_{X}\right)$ for $J^{P C}=2^{-+}$for the case of $\alpha=0$, and $1+3 \cos ^{2}\left(\theta_{X}\right)$ for $J^{P C}=2^{-+}$in case of $\alpha=1$.

Two additional angles are defined as follows: the $x y$-plane is spanned by the kaon direction and the $\pi^{+}$and $\pi^{-}$(back-to-back) directions in the $X(3872)$ rest frame. The $x$-axis is chosen to be along the 
Table 7: $\chi^{2}$ values for the fit of the angular distributions. See text for the definitions of the angles.

\begin{tabular}{lcccc}
\hline Angle & $\chi^{2} /$ n.d.f. & C.L. & $\chi^{2} /$ n.d.f. & C.L. \\
\hline \multicolumn{4}{c}{$J^{P C}=1^{++}$} & \multicolumn{2}{c}{$J^{P C}=2^{-+}$} \\
\hline$\chi$ & $1.76 / 4$ & 0.78 & $4.60 / 4$ & 0.33 \\
$\theta_{\text {lepton }}$ & $0.56 / 4$ & 0.97 & $5.24 / 4$ & 0.26 \\
$\theta_{X}$ & $3.82 / 4$ & 0.51 & $4.72 / 4$ & 0.32 \\
\hline
\end{tabular}

kaon direction. The $z$-axis is constructed perpendicular to the $x y$-plane. The angle $\chi$ is chosen between the $x$-axis and the $\pi^{+}$direction. The angle $\theta_{\mu}$ is chosen between the $\mu^{+}$direction and the $z$-axis. A simultaneous fit for all three angles was performed. The $\chi^{2}$ values are listed in Table 7 . For the case of $J^{P C}=2^{-+}$, the values in Table 7 are given for a complex amplitude $\alpha=0.69 \times \exp \left(i 23^{\circ}\right)$, which was found in a grid search and which is the only value which gives a confidence level $>0.1$ for all three angles. With the available statistics, the two quantum numbers could not be distinguished; however $J^{P C}=1^{++}$ was slightly preferable.

In an analysis by LHCb [123] with a data set of $1.0 \mathrm{fb}^{-1}$, the likelihood with respect to the parameter $\alpha$ was tested, while each event was weighted according to the mass difference $m\left(J / \psi \pi^{+} \pi^{-}\right)-m(J / \psi)$. The likelihood was calculated using five angles, i.e. three helicity angles and two decay plane angles: the polar angle between the $X(3872)$ and the $J / \psi\left(\vartheta_{X}\right)$, the polar angle between the $\pi^{+}$and the $\pi^{+} \pi^{-}$ system $\left(\vartheta_{\pi \pi}\right)$, the polar angle between the $J / \psi$ and the $\mu^{+}\left(\vartheta_{J / \psi}\right)$, the azimuthal angle between the plane spanned by the $\pi^{+}$and the $\pi^{-}$and the plane spanned by the $X$ and the $J / \psi\left(\Delta \phi_{X, \pi \pi}\right)$, and the azimuthal angle between the plane spanned by the $\mu^{+}$and the $\mu^{-}$and the plane spanned by the $X$ and the $J / \psi\left(\Delta \Phi_{X, J / \psi}\right)$. The result strongly favours $J^{P C}=1^{++}$with a confidence level of $34 \%$, while $J^{P C}=2^{-+}$is disfavoured by $8.2 \sigma$, in contrast to the result from the BABAR analysis of the $J / \psi \pi \pi \pi$ decay [159] (see above).

The $J^{P C}=1^{++}$assignment has implications for the production process. The decay $B \rightarrow K X(3872)$ would be $0^{-} \rightarrow 0^{-} 1^{+}$. This means parity $(-1)$ on the left hand side and parity $(-1) \times(+1) \times(-1)^{L}$ for the right hand side. Creating $J=1$ for the $X(3872)$ would require $L=1$, but this implies parity +1 for the right hand side. Consequently it represents a parity violating weak decay.

\subsubsection{Interpretation of the $X(3872)$}

Interpretation as a charmonium state If the $X(3872)$ is a conventional charmonium state, the $J^{P C}=1^{++}$assignment leaves as the only candidate the $\chi_{c 1}^{\prime}, a^{3} P_{1}$ state. The predicted mass by potential models is $m=3953 \mathrm{MeV}$, thus $\simeq 70 \mathrm{MeV}$ higher than the observed $X(3872)$ mass. This would be a $n=2$ radial excitation, and the quantum numbers are favoured by angular analyses [167] [166]. However, there are three arguments against the assignment as the $\chi_{c 1}^{\prime}$ [171]:

- Potential models predict that the mass should be theoretically higher by $\simeq 70 \mathrm{MeV}$. For almost all of the other known charmonium states deviations of the predicted values for the masses and for the widths are $<10 \mathrm{MeV}$.

- The width should be larger with $\simeq 130 \mathrm{MeV}$, compared to the experimental upper limit $\leq 2.3 \mathrm{MeV}$ for the $X(3872)$, as mentioned above.

- The observed ratio of $X(3872) \rightarrow J / \psi \gamma$ to $X(3872) \rightarrow J / \psi \pi^{+} \pi^{-}$is $\simeq 0.18$ (see above). However, for the $\chi_{c 1}^{\prime}$ as a $P$-wave state, radiative decays are expected to be dominant, namely the ratio should be $\geq 40$. 
A notable implication for the potential model is, that the $L S$ term is $-2<1 / r^{3}>$ for the $1^{++}$state, while it would be zero for a $2^{-+}$state. If the $X(3872)$ is the $\chi_{c 1}^{\prime}$, the decay to $\chi_{c 1} \pi^{+} \pi^{-}$should be observable. However, a search by Belle $[172]$ with $711 \mathrm{fb}^{-1}$ was negative. The upper limit on the product branching fraction $\mathcal{B}\left(B^{+} \rightarrow K^{+} X(3872)\right) \times \mathcal{B}\left(X(3872) \rightarrow \chi_{c 1}^{\prime} \pi^{+} \pi^{-}\right)<1.5 \times 10^{6}$ (90\% C.L.) is already a factor $\simeq 5-6$ smaller than $\mathcal{B}\left(B^{+} \rightarrow K^{+} X(3872)\right) \times \mathcal{B}\left(X(3872) \rightarrow J / \psi \pi^{+} \pi^{-}\right)=(8.6 \pm 0.8) \times 10^{-6}$. The same argument would apply, if the $X(3872)$ is not pure charmonium, but an admixture of a tetraquark or molecule with identical quantum numbers, which is disfavored by the small measured upper limit.

An important test for future experiments would be, that there should be a second state nearby, which should exhibit the same mass shift as the $X(3872)$. If the $X(3872)$ is a $J^{P C}=1^{++}$, then it would be $n=2\left({ }^{3} P_{1}\right)$. Then there must be the $h_{c}^{\prime}\left(2^{1} P_{1}, J^{P C}=1^{+-}\right)$nearby ${ }^{8}$

Interpretation as a molecule As mentioned above in Sec. 4.1.1, an intriguing feature of the $X(3872)$ is that its measured mass is very close to the sum of the masses of the $D^{0}$ and $D^{* 0}$ mesons with a mass difference $m(\mathrm{X})-m\left(D^{* 0}\right)-m\left(D^{0}\right)$ of $-0.01 \pm 0.18 \mathrm{MeV}$. This correspondence has led to considerable speculation that the $X(3872)$ is a molecule-like bound state of a $D^{0}$ and $\bar{D}^{* 0}$.

A $J^{P C}=1^{++}$quantum number assignment for the $X(3872)$ implies that $S$-wave couplings of the $X$ to $D^{0} \bar{D}^{* 0}$ is permitted, and these result in a strong coupling between the $X$ and the two mesons. This strong coupling can produce a bound state with a molecular structure just below the two-particle threshold. There are quite a number of arguments in favour of the molecule interpretation, as pointed out by Törnqvist 173]:

- The molecule states should be $J^{P C}=0^{-+}$or $J^{P C}=1^{++}$. For other quantum numbers pion exchange is repulsive or so weak that bound states should not be expected.

- No $D \bar{D}$ are expected since the three pseudoscalar coupling vanishes because of parity.

- If isospin were exact, the $X(3872)$ as a molecule would be a pure isosinglet with a mass very close to the $D \bar{D}^{*}$ threshold, consistent with observation. For isovector states pion exchange is generally one third weaker than for isoscalar states.

- For a state with small binding energy (for comparison, for the deuteron it is $\Delta E_{B}=2.2 \mathrm{MeV}$ ) the Belle state should be large in spatial size. It should then have a very narrow width since annihilation of this loosely bound $D \bar{D}^{*}$ state to other hadrons is expected to be small, although states containing the $J / \psi$ are favoured compared to states with only light hadrons due to the OZI rule.

Even the surprising isospin violation can be explained in the molecule picture; the $D \bar{D}^{*}$ molecule wave function is expected to contain an admixture of $\rho J / \psi$ and $\omega J / \psi$. As in a meson-meson molecular state the long-range parts of the wave function would naively be enhanced, the total width of the state could be larger. An interesting measurement would be, if the partial width of $\Gamma\left(X(3872) \rightarrow J / \psi \pi^{+} \pi^{-}\right)$ is of the order of $\simeq 40 \mathrm{keV}$ (as is predicted for the $\chi_{c 1}^{\prime}$ charmonium state) [174] or $\simeq 202-237 \mathrm{keV}$ (as predicted for for $D^{0 *} \bar{D}^{0}$ molecular state) 174. In any case the total width of a molecule must be larger than the width of its constituent $D^{* 0}$, which is $82.3 \pm 1.2 \pm 1.4 \mathrm{keV}$ [175].

An idea has been proposed [176] to test the molecule hypothesis by comparison of the $X(3872)$ production yields for $B^{0}$ and $B^{+}$decays. Based upon factorization, heavy-quark and isospin symmetries, it was predicted that the neutral/charged ratio has a value

$$
\frac{\mathcal{B}\left(B^{0} \rightarrow K^{0} X(3872)\right)}{\mathcal{B}\left(B^{+} \rightarrow K^{+} X(3872)\right)} \leq 0.1 \text {. }
$$

\footnotetext{
${ }^{8} \mathrm{~T}$. Burns private communication.
} 
In a simplified picture, the reason is that the $B^{0}$ and the $K^{0}$ mesons contain $d$ quarks, but the $B^{+}$, the $K^{+}$and the $D^{0}$ and $\bar{D}^{* 0}$ mesons contain $u$ quarks. This ratio is expected to be unity for charmonium as well as for hybrids (ccg) and glueballs (gg). Belle measured the ratio [162] to $0.82 \pm 0.22 \pm 0.05$ and therefore contradicts the prediction in 176$]$.

Interpretation as a tetraquark Following a suggestion of [177] the $X(3872)$ might represent a tetraquark, in particular in the form with two coloured di-quark pairs. As an indication in favour of the tetraquark interpretation, a mass difference was observed in the two different decay channels

$$
\begin{aligned}
& m\left(J / \psi \pi^{+} \pi^{-}\right)=3871.2 \pm 0.5 \mathrm{MeV} \\
& m\left(D^{0} \bar{D}^{0} \pi^{0}\right)=3875.4 \pm 0.7_{-2.0}^{+1.2} \mathrm{MeV} \\
& m\left(D^{0} \bar{D}^{0} \pi^{0}\right)=3875.6 \pm 0.7_{-1.5}^{+1.4} \mathrm{MeV}
\end{aligned}
$$

which could be regarded as an indication of the possible existence of two different states $X(3872)$ and $X(3876)$. As pointed out in [144], in a tetraquark model these two states could be identified with $X(3872)=[c u][\overline{c u}]$ and $X(3876)=[c d][\bar{c} \bar{d}]$. In a different approach [178], it was proposed to identify the two states $X(3872)$ and $X(3876)$ with the two opposite $G$-parity states $X(3872)=([c q][\overline{c q}]-[c q][\overline{c q}])_{I=0}$ and $X(3876)=([c q][\overline{c q}]+[c q][\overline{c q}])_{I=0}$, where $q$ represents a $u$ or a $d$ quark. The states $[c \bar{c} u \bar{u}]$ and $[c \bar{c} d \bar{d}]$ are contributing with identical weight.

If the $X(3872)$ is a tetraquark, then also charged partners of the form $c \bar{c} u \bar{d}$ and $c \bar{c} d \bar{u}$ should possibly exist. BABAR performed a search in the decay $B \rightarrow K X^{ \pm}, X^{ \pm} \rightarrow J / \psi \pi^{ \pm} \pi^{0}[179]$ with $234 \times 10^{6} B \bar{B}$ events, however with a negative result.

Another search for a $c \bar{c} s \bar{s}$ state has also been performed by $B A B A R$ 180 with $90 \times 10^{6} B \bar{B}$ events and by Belle [181] with $772 \times 10^{6} B \bar{B}$ events. As the $\eta$ meson has an $s \bar{s}$ component in the wave function, there would be sensitivity to this signal according to the tetraquark model. Both searches yielded also a negative result, i.e. no $X(3872)$ signal was observed in this channel.

Interpretation as a threshold effect The constituent quark model as e.g. used in the Cornell potential [17] assumes that the hadronic interaction in the final state is not a significant effect. However, there can be dynamics leading to a modified observed pole position of a resonance. Examples are attraction or repulsion of two resonances or of a resonance and a threshold. A mechanism to generate such a dynamic is the coupled channel approach. As the $X(3872)$ is thus close to the $D^{0} \bar{D}^{0 *}$ threshold, it was suggested that it is a Wigner-cusp [182]. A quantitative estimate [183] shows that the $X(3872)$ is too narrow to be a pure cusp, i.e., the upper limit of the width $\leq 1.2 \mathrm{MeV}$ (see above) must be compared to an expected width of the cusp of $\simeq 15 \mathrm{MeV} \mathrm{I}^{9}$ However, it is possible that the cusp in the real part of the amplitude captures the resonance at the threshold [183], an effect which seems to be observed in the light meson sector as well, e.g. the $f_{0}(980)$ at the $K \bar{K}$ threshold, or the $f_{2}(1565)$ at the $\omega \omega$ threshold. The mixing of the $D^{0} \bar{D}^{0 *}$ threshold and the resonance (decaying into $D^{0} \bar{D}^{0 *}$ ) could be provided by coupling via loop diagrams.

A test scenario was proposed by searching for the partner states. When a $c \bar{c}$ state mixes with $D^{0} \bar{D}^{0 *}$, formally two eigenstates are produced: one gets pulled down by the mixing, but the other is pushed up by level repulsion. The upper one is usually invisible because of its large width. In the case of the $X(3872)$, the second state could be the $Y(3940)$. As the $X(3872)$ and the $Y(3940)$ have a common decay channel, i.e. $J / \psi \omega$, the proposal is intriguing. A disadvantage is that coupling constants for the charmonium states to $D^{0} \bar{D}^{0 *}$ are not at all well known.

A recent unquenched lattice QCD calculation [184] interestingly predicts a $1^{++}$molecule, but no evidence for $1^{-+}$or $1^{--}$molecules. However, the calculated binding energy is $88 \mathrm{MeV}$ and thus too large.

\footnotetext{
${ }^{9}$ D. Bugg private communication.
} 
As an additional note, the $X(3872)$ has implications for the spectrum of conventional charmonium states. Recently the $X(3820)$ 168 has been observed, probably representing the conventional $1^{3} D_{2}$ state with $J^{P C}=2^{--}$. As $J^{P C}=2^{-+}$is excluded for the $\mathrm{X}(3872)$, it can be concluded that a yet unobserved $1^{1} D_{2}$ state with $J^{P C}=2^{-+}$should be close to the $\mathrm{X}(3820) 10$ and possibly to be observed at future experiments.

\subsection{The $X(3940)$ state}

Another new state was observed by Belle in double charmonium production $e^{+} e^{-} \rightarrow c \bar{c} c \bar{c}$. In the reconstruction of the final state a $J / \psi$ is found, and then the recoil mass against the $J / \psi$ is calculated. In this particular production mechanism interestingly the $C$-parity of the recoil (possibly being an $X Y Z$ state) is fixed to $C=+1$. Fig. 27 (left) shows the recoil mass for a data set of $350 \mathrm{fb}^{-1}$. The new state was observed at a mass of $m=3.940 \pm 0.011 \mathrm{GeV}$ with $N=148 \pm 33$ events, corresponding to a statistical significance of $4.5 \sigma$. The other signals in the recoil mass spectrum can be attributed to the conventional charmonium states $\eta_{c}(1 S)$, the $\chi_{c 0}$, and the $\eta_{c}^{\prime}(2 S)$. It is remarkable that only $J=0$ states are observed. As can be seen from Fig. 27 (left), the width is surprisingly narrow, smaller or comparable to the resolution of $32 \mathrm{MeV}$. The background curve is given by a second order polynomial plus a threshold term for $D^{(*)} \bar{D}^{(*)} \mathrm{J} / \psi$. It is interesting to note that no signal of $\mathrm{X}(3872)$ is observed. As in addition to the new $X(3940)$ state three known states with $J=0$ states are observed in the recoil mass spectrum, implying that the new state might also have $J=0$. There are three possible candidate charmonium states:

- The $\eta_{c}^{\prime \prime}(3 S)\left(3^{1} \mathrm{~S}_{0}, J^{P C}=0^{-+}\right)$has a $\simeq 100 \mathrm{MeV}$ higher predicted mass of $m=4064 \mathrm{MeV}[171]$. Predictions of static potential models should be more accurate in particular for the case $S=0$ and $L=0$.

- The $h_{c}\left(2^{1} \mathrm{P}_{1}, J^{P C}=1^{+-}\right)$is expected around 3934-3956 MeV [171]. However, states with negative $C$-parity (such as the lower $h_{c}$ ) are not visible.

- The identification as the $\chi_{c 0}^{\prime}$ is not preferred either, as the $\chi_{c 2}^{\prime}$ was observed at $\simeq 3930$, and due to the spin-spin forces the $J=0$ state should be $63 \mathrm{MeV}$ lower than the $J=2$ state.

The tentative conclusion is that the $X(3940)$ is probably not a charmonium state. An interesting additional question is, if the $X(3940)$ and the $X(3915)$ may be the same state, as the latter one is presently a strong candidate for the conventional charmonium state $\chi_{c 0}^{\prime}$. For the $X(3915)$ an upper limit of $\mathcal{B}(B \rightarrow X(3915) K) \times \mathcal{B}\left(X(3915) \rightarrow D^{* 0} \bar{D}^{0}\right)<0.67 \times 10^{-4}$ at $90 \% \mathrm{CL}$ was measured $\mid 134$. By averaging the branching fractions of [185] and [158], one obtains $\mathcal{B}(B \rightarrow X(3915) K) \times \mathcal{B}(X(3915) \rightarrow$ $\omega J / \psi)=(0.51 \pm 0.11) \times 10^{-4}$. Combining the two numbers, one gets the ratio $\mathcal{B}(X(3915) \rightarrow \omega J / \psi)$ $/ \mathcal{B}\left(X(3915) \rightarrow D^{* 0} \bar{D}^{0}\right)>0.71$ at $90 \% \mathrm{CL}$. This must be compared with the $90 \%$ CL limits from $\mathcal{B}(X(3940) \rightarrow \omega J / \psi)<0.26$ and $\mathcal{B}\left(X(3940) \rightarrow D^{* 0} \bar{D}^{0}\right)>0.45$, and thus the ratio of the two branching ratios $\mathcal{B}(X(3940) \rightarrow \omega J / \psi) / \mathcal{B}\left(X(3940) \rightarrow D^{* 0} \bar{D}^{0}\right)<0.58$ at $90 \%$ CL 186 . This incompatibility suggests that the $X(3940)$ and the $X(3915)$ are different states.

In a further study [187], the analysis was extended to additional specification and identification of the recoil particles, using a data set of $693 \mathrm{fb}^{-1}$. The technique uses one fully reconstructed $D^{(*)}$ meson, which is denoted as $D_{\text {rec }}$ in Fig. 27 (right). The $D^{*}$ mesons are reconstructed via their decays $D \pi$. The $D$ mesons are reconstructed in the five decay channels $K^{-} \pi^{+}, K^{-} K^{+}, K^{-} \pi^{-} \pi^{+} \pi^{+}, K_{s}^{0}\left(\rightarrow \pi^{+} \pi^{-}\right) \pi^{+} \pi^{-}$ and $K^{-} \pi^{+} \pi^{0}(\rightarrow \gamma \gamma)$. The second $D^{(*)}$ meson is then identified by using the recoil mass against the $J / \psi D_{\text {rec }}$ system. All of the observed final states seem to exhibit an $S$-wave enhancement.

\footnotetext{
${ }^{10}$ T. D. Burns private communication.
} 

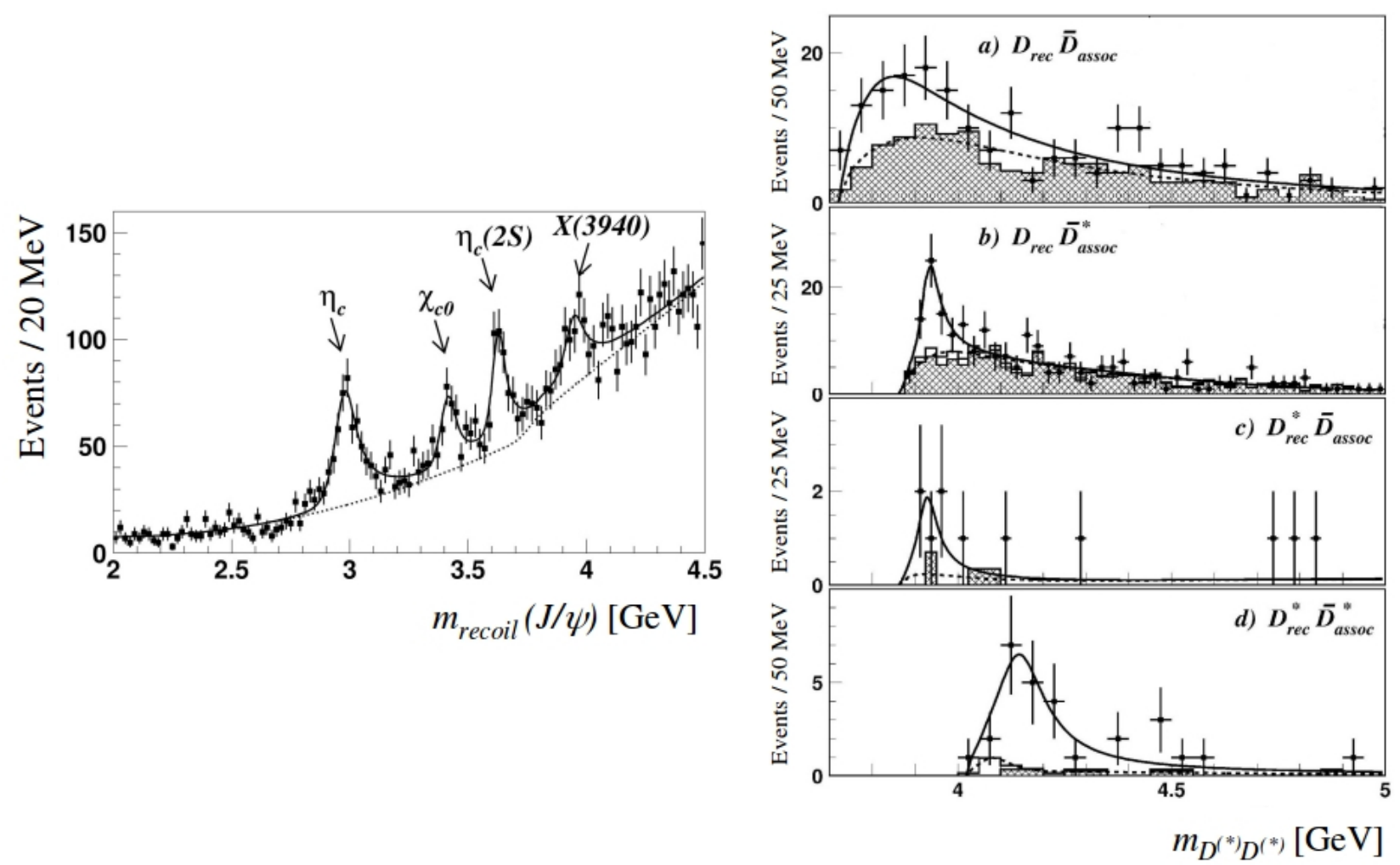

Figure 27: Left: Recoil mass for double charmonium production from Belle [186]. The new state $X(3940)$ is visible, along with the known states $\eta_{c}(1 S)$, the $\chi_{c 0}$ and the $\eta_{c}^{\prime}(2 S)$. Right: $D \bar{D}(a), D \bar{D}^{*}$ (b), $D^{*} \bar{D}(c)$ and $D^{*} \bar{D}^{*}(d)$ invariant mass distributions of mesons recoiling against a $J / \psi$ in double charmonium production from Belle [187]. In $(d)$, the new state $X(4140)$ is visible, which has a preferred charge conjugation assignment of $C=+1$.

Fig. 27 (right, (a)) shows the $D \bar{D}$ invariant mass recoiling against a $J / \psi$ in double charmonium production. The $X(3880)$ resonance is visible, although only with a $3.8 \sigma$ statistical significance. As the fit is not stable under the variation of background parametrization, no observation was claimed. Fig. 27 (right, (b) and (c)) show the $\bar{D} D^{*}$ invariant mass recoiling against a $J / \psi$ in double charmonium production, in the two different cases of either the $\bar{D}$ or the $D^{*}$ side reconstructed. The $X(3940)$ (mentioned above) is visible with a statistical significance of $6.0 \sigma$. The mass and the width were determined as $3942_{-6}^{+7} \pm 6 \mathrm{MeV}$ and $37_{-15}^{+26} \pm 8 \mathrm{MeV}$, respectively. Note that the $X(3940)$ is only observed here in $D^{*} \bar{D}$ and not in $D \bar{D}$, giving preliminary preference to an assignment of $J=1$ (because of the $D^{*}$ ) instead of $J=0$. In addition, a decay to $J / \psi \omega$ was checked and not observed, importantly indicating that the $X(3940)$ and the $Y(3940)$ (see Sec. 4.2) are not the same.

Fig. 27 (right, (d)) shows the $D^{*} \bar{D}^{*}$ invariant mass recoiling against a $J / \psi$ in double charmonium production. Another state, tentatively called $X(4140)$, is visible with a statistical significance of $5.5 \sigma$ $\left(N=24 \pm 5\right.$ events). From the fit, a mass of $4156_{-20}^{+25} \pm 15 \mathrm{MeV}$ and a width of $139_{-61}^{+111} \pm 21 \mathrm{MeV}$ were determined. A calculation of $D^{*} \bar{D}^{*}$ molecules predicts a tensor state $J^{P C}=2^{++}$state at this mass. ${ }^{11}$ This hypothesis could be tested by searching for vector vector decays such as $K^{*} \bar{K}^{*}$, $\omega \omega$ or $\phi \phi$ which are predicted to have a significant branching, although these decays would be $\mathrm{SU}(3)$ violating.

Concerning the quantum numbers of the new states, in the case of no other final state particle (i.e. exclusive $J / \psi D^{*} \bar{D}^{*}$ ), the charge conjugation $C=+1$ value is preferred. $C=+1$ charmonium states are interesting, as they cannot annihilate to a virtual photon. Thus they cannot decay to $e^{+} e^{-}$or $\mu^{+} \mu^{-}$, but only to $\gamma \gamma$ or two gluons. Interestingly, the decay widths such as for the decay ${ }^{3} \mathrm{P}_{0} \rightarrow \gamma \gamma$ are

${ }^{11} \mathrm{E}$. Oset private communication. 
Table 8: Possible molecular $D^{(*)} \bar{D}^{(*)}$ states as predicted in Ref. 173.

\begin{tabular}{llc}
\hline Constituents & $J^{P C}$ & Mass $(\mathrm{MeV})$ \\
\hline$D \bar{D}^{*}$ & $0^{-+}$ & $\simeq 3870$ \\
$D \bar{D}^{*}$ & $1^{++}$ & $\simeq 3870$ \\
\hline$D^{*} \bar{D}^{*}$ & $0^{++}$ & $\simeq 4015$ \\
$D^{*} \bar{D}^{*}$ & $0^{-+}$ & $\simeq 4015$ \\
$D^{*} \bar{D}^{*}$ & $1^{+-}$ & $\simeq 4015$ \\
$D^{*} \bar{D}^{*}$ & $2^{++}$ & $\simeq 4015$ \\
\hline
\end{tabular}

proportional not to the squared wave function at the origin, but to the squared derivative of the wave function $|\partial \psi / \partial r(r=0)|^{2}$. Thus $C=+1$ states represent a precise tool to study the exact wave function behavior for $r \rightarrow 0$.

The relevant threshold for the new state is given by $\mathrm{m}\left(D^{*+}\right)+\mathrm{m}\left(D^{*-}\right) \simeq 4020 \mathrm{MeV}$. Thus, the new state has a positive mass difference of $\Delta m \simeq 120 \mathrm{MeV}$, or, in other words, the binding energy has the wrong sign. The dynamics, which could create a state with such a large $\Delta m$ is not clear yet. The two new states $X(3940)$ and $X(4140)$ are not yet confirmed by any other experiment.

The observed states can be compared to predictions for molecular states based upon a one-pion exchange model [173] which are listed in Table 8. The observed masses are higher than the predicted masses, e.g. by $\simeq 125 \mathrm{MeV}$ for the $X(4140)$ if compared to the hypothetical $0^{++}$state with positive charge conjugation. As the difference is positive, it can not be interpreted as a binding energy. Instead, if the molecular hypothesis is correct, the states would be virtual states above threshold.

\section{$4.3 \quad Y$ states}

Another new charmonium-like state was discovered by BABAR 188 with a data set of $211 \mathrm{fb}^{-1}$. The production process is given by initial state radiation $e^{+} e^{-} \rightarrow \gamma_{I S R} J / \psi \pi^{+} \pi^{-}$. A photon is radiated by either the $e^{+}$or the $e^{-}$in the initial state, lowering $\sqrt{s}$ and producing the $Y(4260)$. The state was observed in the invariant mass $m\left(J / \psi \pi^{+} \pi^{-}\right)$. Based upon this production process, in which $e^{+} e^{-} \rightarrow$ $\gamma_{I S R} \gamma_{V}, \gamma_{V} \rightarrow Y(4260)$, the quantum numbers are $J^{P C}=1^{--}$. Historically, the tentative name $Y$ was assigned, which is typical for vector states and similar to $\Upsilon$ or $\psi$. The mass was measured as $4259 \pm 8_{-6}^{+2} \mathrm{MeV}, \simeq 500 \mathrm{MeV}$ above the $D \bar{D}$ threshold. The width was determined as $88 \pm 23_{-4}^{+6} \mathrm{MeV}$. Thus, the state is significantly broader than the $X(3872)$, but still surprisingly narrow considering its high mass. The state was confirmed by Belle with a data set of $553.2 \mathrm{fb}^{-1}[189]$. However, the mass was measured to be $\simeq 2.5 \sigma$ higher than the mass measured by $B A B A R$, and the width $\simeq 50 \%$ wider than in the case of $B A B A R$. Later both experiments updated their results [189 191].

Figure 28 shows $Y(4260)$ signals for Belle and BABAR. As can be seen in the invariant mass $m\left(J / \psi \pi^{+} \pi^{-}\right)$spectra, the background shapes are somewhat different between the experiments. The reason is the different design of the interaction regions in the two experiments, namely BABAR had collisions head-on, which required a dipole magnet very close to the interaction point generating additional background. Belle used a steering angle between both beams, not requiring magnets close to the interaction point. As can also be seen in Fig. 28, the lineshape of the $Y(4260)$ shows a long range tail at high masses. This particular lineshape results not from radiative effects, but from a strong dependance of the reconstruction efficiency as a function of the invariant mass $m\left(J / \psi \pi^{+} \pi^{-}\right)$. The fit function applied in e.g. [189] is a Breit-Wigner function folded with a phase space ${ }^{12}$ term and efficiency

\footnotetext{
${ }^{12}$ Note that the energy dependent width $\Gamma(s)$ corresponds to a phase space increase, however the $Y(4260)$ is regarded as being arrow and this $\Gamma$ is chosen independent of $s$. Thus, in the Belle analyses of the Y(4260) [189 an additional phase
} 

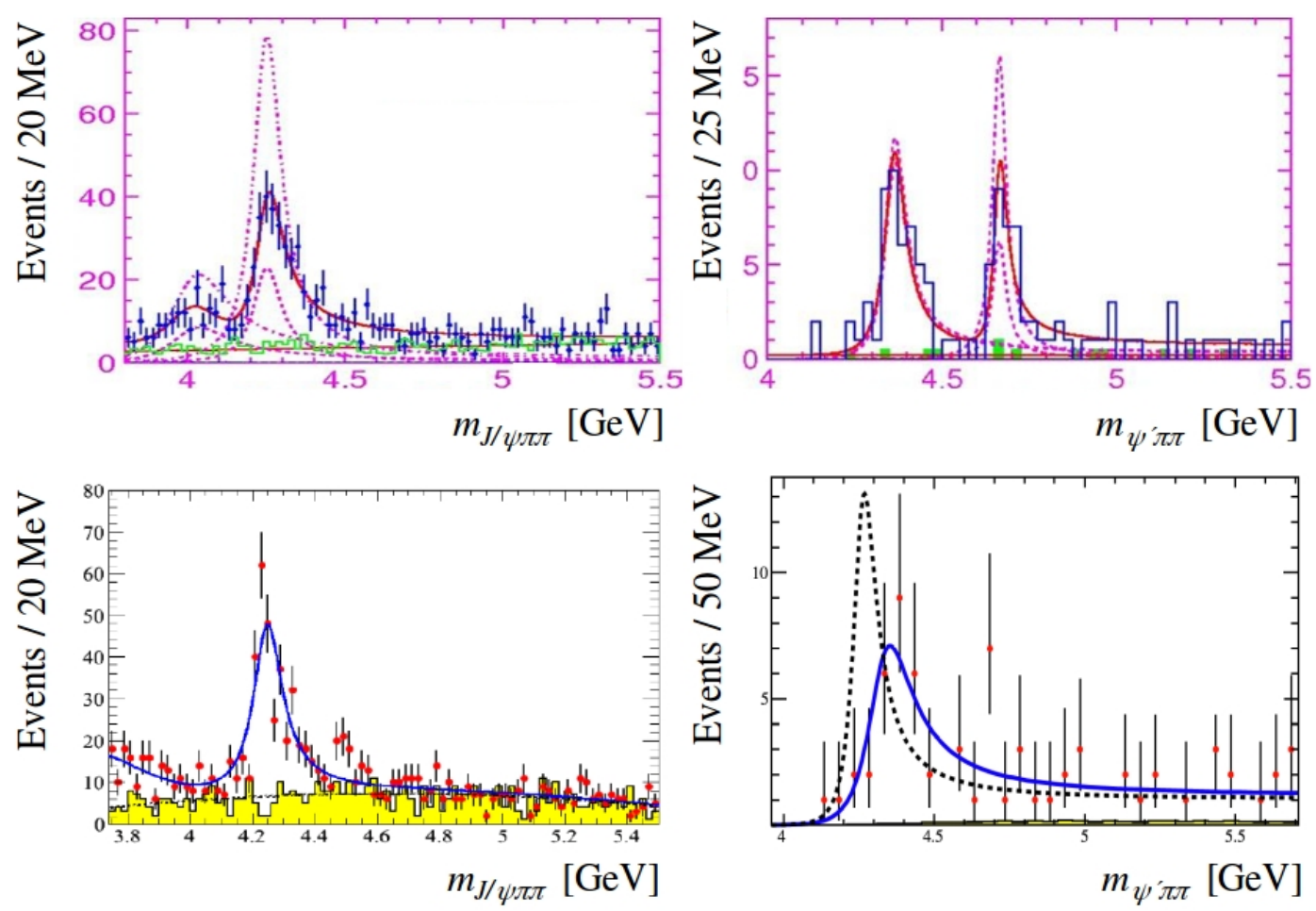

Figure 28: Summary of the $Y$ states observed in ISR. Top left: Analysis of $e^{+} e^{-} \rightarrow \gamma_{I S R} J / \psi \pi^{+} \pi^{-}$with a data set of $550 \mathrm{fb}^{-1}$ from Belle [192]. Top right: Analysis of $e^{+} e^{-} \rightarrow \gamma_{I S R} \psi^{\prime} \pi^{+} \pi^{-}$with a data set of $670 \mathrm{fb}^{-1}$ from Belle [193]. Bottom left: Analysis of $e^{+} e^{-} \rightarrow \gamma_{I S R} J \psi \pi^{+} \pi^{-}$with a data set of 454 $\mathrm{fb}^{-1}$ from BABAR [190]. Bottom right: Analysis of $e^{+} e^{-} \rightarrow \gamma_{I S R} \psi^{\prime} \pi^{+} \pi^{-}$with a data set of $298 \mathrm{fb}^{-1}$ from BABAR [194]. Dotted and dash-dotted lines indicate solutions of the fits including and excluding interference.

$\varepsilon$, which is parametrised as $\varepsilon=a\left(m-m_{0}\right)+b$ with $a=7.4 \pm 1.3 \mathrm{GeV}^{-1}$ and $b=9.31 \pm 0.07$, i.e., the efficiency changes by a factor $\simeq 2$ over the peak region.

In an independent additional measurement, the $Y$ (4260) was confirmed by CLEO-c [195] using a scan, i.e. by variation of the beam energies. The measurements of all three experiments are summarized in Table 9. CLEO-c also reported a $5.1 \sigma$ evidence for a second decay channel $Y(4260) \rightarrow J / \psi \pi^{0} \pi^{0}[196]$, which was confirmed by Belle [197]. The ratio of the two branching fractions was determined as $\mathcal{B}\left(J / \psi \pi^{0} \pi^{0}\right) / \mathcal{B}\left(J / \psi \pi^{+} \pi^{-}\right) \simeq 0.5$. This leads to the important conclusion, based upon the ClebschGordan coefficients in isospin space, that the isospin of the $\pi \pi$ system must be zero, i.e., $I^{G}=0^{+}$. By symmetry arguments, this leads to $J^{P C}=0^{++}$or $2^{++}$for the $\pi \pi$ system. BABAR investigated the $\pi^{+} \pi^{-}$ invariant mass distribution [188]. The observed angular distribution is consistent with $\mathrm{J}^{P C}=0^{++}$, i.e. consistent with $\pi^{+} \pi^{-}$phase space for $S$-wave $J / \psi \pi^{+} \pi^{-}$. Note, in particular, that this is completely different from the $X(3872)$, where the $\pi^{+} \pi^{-}$system is in a $\mathrm{J}^{P C}=1^{--}$state with $I=1$. The $Y(4260)$ does not violate isospin in the decay.

In addition, CLEO-c reported a $3.7 \sigma$ evidence for a third decay channel, namely $Y(4260) \rightarrow J / \psi K^{+} K^{-}$ [196], which unfortunately could not be confirmed by Belle [198]. Searches were also performed for the $Y(4260)$ in $B$ meson decays [199], however the phase space is small and the results are compatible with statistical fluctuations.

space term is applied. 
Table 9: Summary of the mass and width measurements of the $Y(4260)$.

\begin{tabular}{lllllll}
\hline & BABAR $[188$ & CLEO-c $[195]$ & Belle $[189]$ & Belle $[192]$ & BABAR $\mid 190]$ & BABAR $[191]$ \\
\hline $\mathcal{L}$ & $211 \mathrm{fb}^{-1}$ & $13.3 \mathrm{fb}^{-1}$ & $553 \mathrm{fb}^{-1}$ & $548 \mathrm{fb}^{-1}$ & $454 \mathrm{fb}^{-1}$ & $454 \mathrm{fb}^{-1}$ \\
$\mathrm{~N}$ & $125 \pm 23$ & $14.1_{-4.2}^{+5.2}$ & $165 \pm 24$ & $324 \pm 21$ & $344 \pm 39$ & - \\
Significance & $\simeq 8 \sigma$ & $\simeq 4.9 \sigma$ & $\geq 7 \sigma$ & $\geq 15 \sigma$ & - & - \\
$m(\mathrm{MeV})$ & $4259 \pm 8_{-6}^{+2}$ & $4283_{-16}^{+17} \pm 4$ & $4295 \pm 10_{-3}^{+10}$ & $4247 \pm 12_{-32}^{+17}$ & $4252 \pm 6_{-3}^{+2}$ & $4244 \pm 5 \pm 4$ \\
$\Gamma(\mathrm{MeV})$ & $88 \pm 23_{-4}^{+6}$ & $70_{-25}^{+40}$ & $133 \pm 26_{-6}^{+13}$ & $108 \pm 19 \pm 10$ & $105 \pm 18_{-6}^{+4}$ & $114_{-15}^{+16} \pm 7$ \\
\hline
\end{tabular}

Table 10: Summary of the mass and width measurements of the $Y(4350)$.

\begin{tabular}{llll}
\hline & Yield & $m(\mathrm{MeV})$ & $\Gamma(\mathrm{MeV})$ \\
\hline BABAR 194 & $298 \mathrm{fb}^{-1}$ & $4324 \pm 24$ & $172 \pm 33$ \\
Belle 193 & $673 \mathrm{fb}^{-1}$ & $4361 \pm 9 \pm 9$ & $74 \pm 15 \pm 10$ \\
\hline
\end{tabular}

The $\pi^{+} \pi^{-}$mass distribution in $Y(4260) \rightarrow J \psi \pi^{+} \pi^{-}$exhibits an $f_{0}(980)$ signal [191] with $J^{P C}=0^{++}$ (positive parity). This is clearly different from the $X(3872)$, in which the $\pi^{+} \pi^{-}$system exhibits a $\rho$ signal (see Sec. 4.1 with a $J^{P C}=1^{--}$(negative parity), and is a consequence of the different quantum numbers of the $Y(4260)$ (negative parity) and the $X(3872)$ (positive parity) under the assumption of $S$-wave decays.

Belle reported a second state with $\simeq 250 \mathrm{MeV}$ lower mass: the $Y(4008)$. The width is reported to be about a factor $\simeq 2$ larger than that of the $Y(4260)$. This state so far was not confirmed by any other experiment. Another important result of the Belle analysis is that no evidence for any higher mass state up to $7 \mathrm{GeV}$ was found. There has also been the attempt to quantify the contribution of higher $\psi$ resonances $(\psi(3 \mathrm{D}), \psi(5 S), \psi(4 D), \psi(6 S)$ and $\psi(5 D))$ to the $J / \psi \pi \pi^{-}$spectrum [200].

In 2007 initial state radiation processes with a $\psi^{\prime}$ instead of a $J / \psi$ were investigated by $B A B A R$, and another new state was found in the $\psi^{\prime} \pi^{+} \pi^{-}$invariant mass (see Fig. 28) in a data sample of $298 \mathrm{fb}^{-1}$. The new resonance, tentatively called $Y(4350)$, has a peak cross section of $\simeq 80 \mathrm{pb}^{-1}$, almost as large as the $Y(4260)$. However, the peak position at $4324 \pm 24 \mathrm{MeV}$ is significantly different from the mass of the $Y(4260)$, so that it must be concluded for now that these are two different states. The width was measured to be $172 \pm 33 \mathrm{MeV}$. Belle was able to confirm the $Y(4350)$ [193] (Fig. 28). The mass and the width measurements are summarized in Table 10.

In the analysis of the $Y(4350)$ Belle used a factor $\simeq 3$ larger data sample of $670 \mathrm{fb}^{-1}$, which also revealed evidence for another new state $Y(4660)$ with a statistical significance of $5.8 \sigma$. The measured mass and width are $4664 \pm 11 \pm 5 \mathrm{MeV}$ and $48 \pm 15 \pm 3 \mathrm{MeV}$, respectively. The $Y(4660)$ is the heavyist charmonium-like state ever observed, with a mass of $\simeq 1.5 \mathrm{GeV}$ above the charmonium ground state.

Interpretation as a charmonium state All the $Y$ states have to carry the quantum numbers $J^{P C}=1^{--}$, due to their observation in an ISR process. There are five known and assigned $J^{P}=1^{--}$ charmonium states: $J / \psi, \psi(2 S), \psi(4040), \psi(4160)$ and $\psi(4415)$. As we will see there is a clear overpopulation of $1^{--}$states in the $m \geq 4 \mathrm{GeV}$ region. Somewhat surprisingly, although they are partially overlapping due to their widths, apparently there seems to be no mixing:

- the $Y(4008)$ candidate state and the $Y(4260)$ decay to $J / \psi \pi^{+} \pi^{-}$, and the $Y(4350)$ and the $Y(4660)$ decay to $\psi^{\prime} \pi^{+} \pi^{-}$, and neither of one has been observed in the other channel yet, not even indirectly as a distortion of the lineshape, and

- no mixing of the $\psi$ states with the $Y$ states was observed so far. 
Mass / GeV

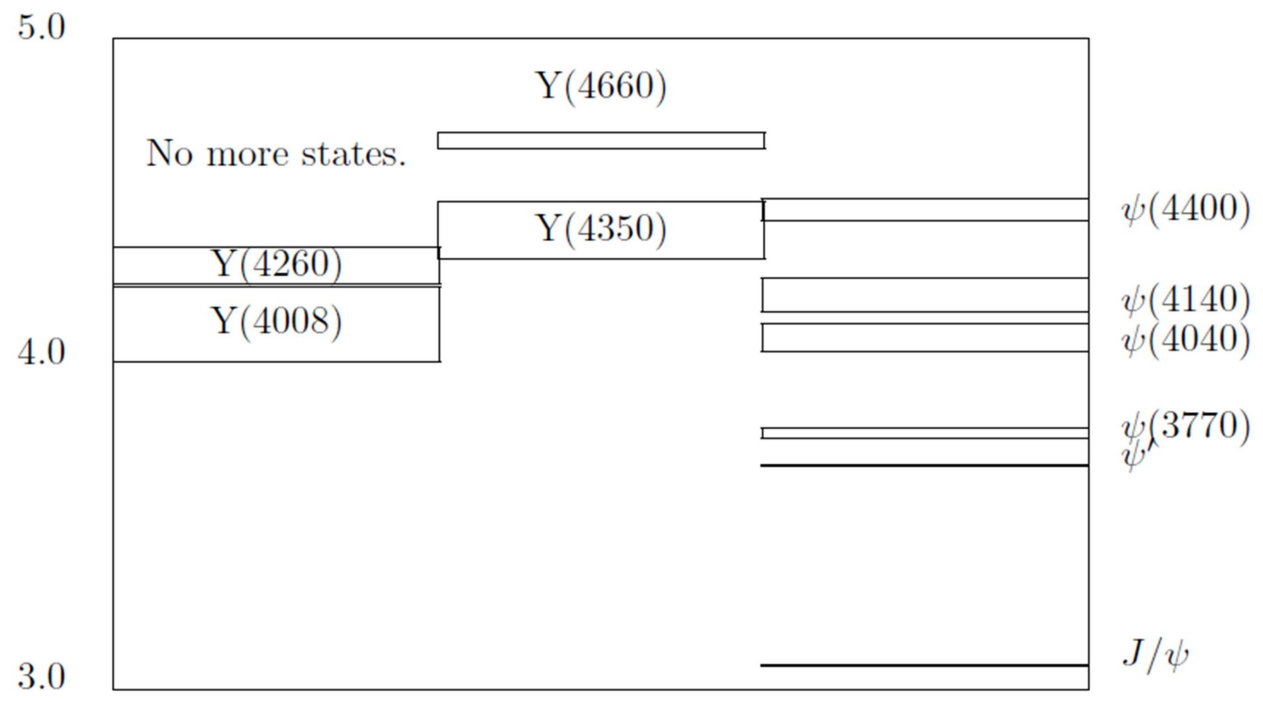

Figure 29: Mass level scheme of the observed $Y$ states in comparison to the known excited $\psi(n S)$ states All of them carry the quantum numbers $J^{P}=1^{--}$, but due to the experimental observation they are separated in three different columns: states observed in ISR which decay into $J / \psi \pi^{+} \pi^{-}$(left), states observed in ISR which decay into $\psi^{\prime} \pi^{+} \pi^{-}$(center) and the known $\psi$ states (right). The vertical sizes of the boxes indicate their widths.

On the other hand, there is evidence of destructive interference from the analysis of the known $\psi$ states in the region $4.2 \leq m \leq 4.4 \mathrm{GeV}$ [201]. In particular, the $e^{+} e^{-}$cross section shows a local minimum in the $Y(4260)$ mass region, pointing to destructive interference of the $\psi$ states with the $Y(4260)$. The pattern of the $Y$ states appears non-trivial (see Fig. 29): two non-mixing doublets without parity flip and without charge flip. It remains unclear what the underlying symmetry is. In addition, there is no obvious pattern so far, as to how the masses of the $\psi$ states and the masses of the $Y$ states might be related.

Intriguingly, the $Y(4260)$ state is again close to a threshold 165 . The $\bar{D}^{0} D_{1}(2420)$ threshold is at $4286.3 \mathrm{MeV}$ with a summed narrow width of the constituent mesons of $\simeq 27 \mathrm{MeV}$, and thus the $Y(4260)$ has been discussed as a molecule [202]. The quantum numbers of $J^{P}=0^{-}$and $J^{P}=1^{+}$can be combined correctly in a relative $S$-wave to the observed $J^{P}=1^{-}$. The threshold would be in the tail of the $Y(4260)$, however a modified line shape is not observed. A second nearby threshold is $D^{o} \bar{D}_{2}^{* o}(2460)$ at $4327.5 \mathrm{MeV}$ with a summed width of the constituent mesons of $\simeq 49 \mathrm{MeV}$. However, a molecule would not be an option for the $Y(4260)$ due to the $J^{P}=2^{+}$of the $\bar{D}_{2}^{* o}(2460)$, i.e. an $S$-wave coupling would lead to $J^{P}=2^{-}$, but not $J^{P}=1^{-}$.

The $Y(4260)$ has also been discussed in literature as a hybrid $\left[c \bar{c}_{8} g\right]$ with a color octet $c \bar{c}$ pair bound to a valence type gluon. However, recently there is evidence that the $Y(4260)$ decays also to $h_{c} \pi^{+} \pi^{-}$[203], which would imply a spin flip of the heavy quark system. If this decay is confirmed by another measurement, an interpretation of the $Y(4260)$ as a hybrid would be strongly disfavoured. As discussed in the section on theoretical models, a similar pattern is observed in the decays $\Upsilon(5 S) \rightarrow \Upsilon(n S) \pi^{+} \pi^{-}, n S=1 S, 2 S, 3 S$, and $\Upsilon(5 S) \rightarrow\left(h_{b}(1 P), h_{b}(2 P)\right) \pi^{+} \pi^{-}$, which are dominated by the intermediate $Z_{b}(10650)^{+} \pi^{-}$and $Z_{b}(10610)^{+} \pi^{-}$states. In the tetraquark interpretation, the two $Z_{b}$ states have both $b \bar{b}$ (spin-0) and $b \bar{b}$ (spin-1) components in their Fock space. Presumably, the same phenomenon is at work in the decays of $Y(4260)$.

A state which is probably identical to the $Y(4660)$ has also been observed at Belle [204] in the ISR production process using a data set of $670 \mathrm{fb}^{-1}$, but in a different decay channel, i.e. the signal was 


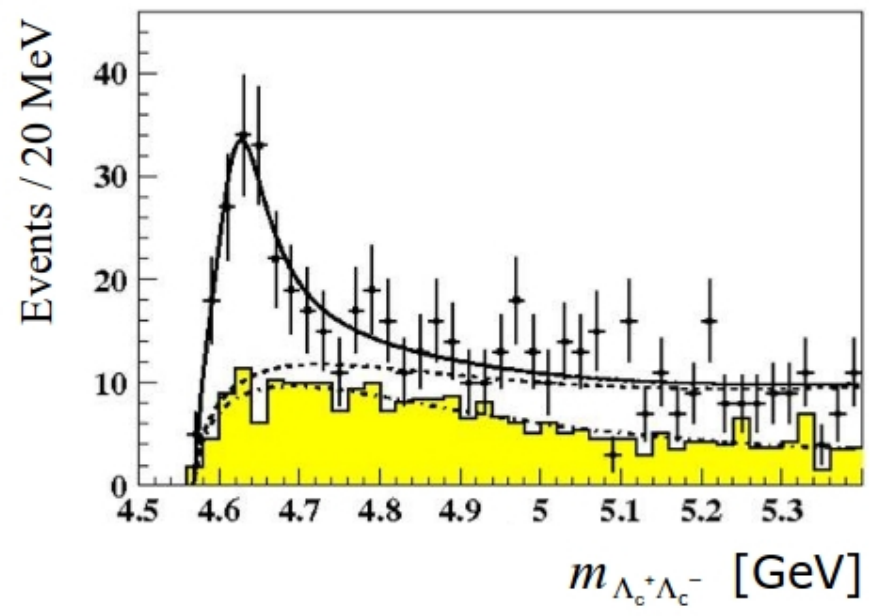

Figure 30: Invariant mass $m\left(\Lambda_{c}^{+} \Lambda_{c}^{-}\right)$for the process $e^{+} e^{-} \rightarrow \gamma_{I S R} \Lambda_{c}^{+} \Lambda_{c}^{-}$from Belle [204] showing the signal for the $X(4630)$.

observed in $e^{+} e^{-} \rightarrow \gamma_{I S R} \Lambda_{c}^{+} \Lambda_{c}^{-}$. The state is usually referred to as the $X(4630)$. The $\Lambda_{c}^{+}$is reconstructed in the final states $p K_{s}^{0}\left(\rightarrow \pi^{+} \pi^{-}\right), p K^{-} \pi^{+}$, and $\Lambda\left(\rightarrow p \pi^{-}\right) \pi^{+}$. For the $\Lambda_{c}^{-}$only partial reconstruction is used: The recoil mass to $\left[\Lambda_{c}^{+} \gamma\right]$ is investigated while requiring an anti-proton (from the $\Lambda_{c}^{-}$decay) as a tag and then a cut around the $\Lambda_{c}^{-}$mass is applied. The measured mass is $4634_{-7-8}^{+8+5} \mathrm{MeV}$ and the measured width is $92_{-24-21}^{+40+10} \mathrm{MeV}$. Figure 30 shows the invariant mass $m\left(\Lambda_{c}^{+} \Lambda_{c}^{-}\right)$. A signal with a statistical significance of $8.2 \sigma$ is observed. The observation of this state is remarkable because of two reasons: it represents the highest charmonium-like state observed so far (along with the $Y(4660)$ of almost same mass, but decaying into $J / \psi \pi^{+} \pi^{-}$), and it is the only $X Y Z$ state so far observed decaying into baryons.

\subsection{Z states of type $\mathrm{I}$ in the charmonium mass regime}

The first of the charged charmonium-like states was first observed by Belle in the decay channel $B^{0} \rightarrow$ $\psi^{\prime} K^{ \pm} \pi^{\mp}$. We will refer to this state, and similar states observed in $B$ meson decays, as $Z$ states of type I. Since it was observed in the charmonium mass regime, and decays to final states with open or closed charm, we will also use the short form $Z_{c}$. A Dalitz plot of this decay channel is shown along with the $\psi^{\prime} \pi^{\mp}$ squared invariant mass projection in Fig. 31 (top). The peak in the mass spectrum is due to the $Z_{c}^{+}(4430)$. The existence of this resonance is remarkable, as it is charged, while a charmonium state must be neutral. In the analysis it is important to take into account resonances in the $K^{ \pm} \pi^{\mp}$ channel, in order to solely extract the dynamics in the $\psi^{\prime} \pi^{\mp}$ channel. In the Dalitz plot in Fig. 31 resonances in the $K^{ \pm} \pi^{\mp}$ channel are seen as vertical bands, resonances in $\psi^{\prime} \pi^{\mp}$ as horizontal bands. Two vertical bands, corresponding to the $K^{*}(892)$ and the $K_{2}^{*}(1430)$ are visible and expected. In Fig. 31 (top right), a veto on both $K^{*}$ resonances is applied. The first observation of this state [205] with a data set of $605 \mathrm{fb}^{-1}$ showed a statistical significance of $6.5 \sigma$.

The resonance parameters were determined from a fit to $\psi^{\prime} \pi^{+}$mass spectrum giving a mass of $4433 \pm 4 \pm 2 \mathrm{MeV}$ and a width of $45_{-13-13}^{+18+30} \mathrm{MeV}$. This is quite narrow for a state with such a high mass. $B A B A R$ found contrary evidence for the $Z_{c}^{+}(4430)$ with a data set of $413 \mathrm{fb}^{-1}$ [110]. The argument was based upon the observation that the kinematic variables in this final state are strongly correlated. Particular importance was attributed to $\cos \theta_{K}$, the normalized dot-product between the $K \pi$ threemomentum vector in the parent- $B$ rest frame and the kaon three-momentum vector after a Lorentz transformation from the $B$ meson rest frame to the $K \pi$ rest frame. This parameter $\cos \theta_{K}$ is correlated with $m\left(K^{ \pm} \pi^{\mp}\right)[110]$. A forward/backward asymmetry is observed, i.e. $\cos \theta_{K}<0$ is preferred for the $K^{*}(892)$ and the $K_{2}^{*}(1430)$. This effect can produce structures in the $m^{2}\left(\psi^{\prime} \pi^{\mp}\right)$ invariant mass in the 
high mass region, depending on applied $K^{*}$ cuts in the analysis. The BABAR analysis yields only a $2.7 \sigma$ statistical significance for the $Z_{c}^{+}(4430)$.

In a re-analysis [206] of the original Belle observation in [205] the $Z_{c}^{+}$(4430) was confirmed usin a 2-dimensional fit of the Dalitz plot (Fig. 31, top left). The resulting statistical significance is 6.4 $\sigma$. Note that both charged states $Z_{c}^{+}$and $Z_{c}^{-}$are observed. If these $Z$ states would turn out to carry isospin, this would have the important implication of the $Z^{ \pm}$being an iso-doublet.

In a $3 \mathrm{fb}^{-1}$ data set recorded at $\sqrt{s}=7$ and $8 \mathrm{TeV}$, LHCb finally confirmed this state with a very large significance $\geq 13.9 \sigma \mid 209]$. The Dalitz plot and the projected squared mass distribution are shown in Fig. 31 (bottom). The full amplitude analysis used a 4-dimensional Dalitz fit of two squared masses $m_{K \pi}^{2}$ and $m_{\psi^{\prime} \pi}^{2}$, and two angles $\theta_{\psi^{\prime}}$ (the $\psi^{\prime}$ helicity angle, defined as the angle between the momenta of the $\left(K^{+} \pi^{-}\right)$system and the $\mu^{-}$in the $\psi^{\prime}$ rest frame), and $\phi$ (the angle between the planes defined by the $\mu^{+} \mu^{-}$and $K^{ \pm} \pi^{\mp}$ systems). The quantum numbers of the $Z_{c}^{ \pm}(4430)$ were unambiguously established as $J^{P}=1^{+}$, excluding $0^{-}, 1^{-}, 2^{+}$, and $2^{-}$by $9.7 \sigma, 15.8 \sigma, 16.1 \sigma$ and $14.6 \sigma$. The measured mass and width are consistent with the Belle determination.

A second $Z$ state with a significance $6 \sigma$ was also observed with a mass of $4239 \pm 18_{-10}^{+45} \mathrm{MeV}$ and a width of $220 \pm 47_{-74}^{+108} \mathrm{MeV}$. In the fit, $J^{P}=0^{-}$was preferred over the $J^{P}=1^{-}, 2^{-}$, and $2^{+}$by $8 \sigma$. The $0^{+}$ interpretation was preferred over the $1^{+}$by only $1 \sigma$, however, in a fit with $J^{P}=1^{+}$the width of $Z_{c}(4430)$ would also increase by $660 \mathrm{MeV}$, not being consistent anymore with the Belle measurement.

The observation of a charged state in the decay $B^{+} \rightarrow J / \psi \pi^{+} K^{-}$, tentatively called the $Z_{c}(4200)$, was made by Belle using the full data set of $711 \mathrm{fb}^{-1}$ [208], with a measured mass of $4196_{-29-13}^{+31+17} \mathrm{MeV}$ and a width of $370_{-70-132}^{+70+70} \mathrm{MeV}$. The Dalitz plot and projected squared mass distribution are shown in Fig. 31 (second row). The significance was $6.2 \sigma$. Again, $J^{P}=1^{+}$was preferred with an exclusion level of $6.1 \sigma, 7.4 \sigma, 4.4 \sigma, 7.0 \sigma$ for $J^{P}=0^{-}, 1^{+}$, and $2^{-}, 2^{+}$. Note, $0^{+}$is forbidden by parity conservation.

Another very important result of this analysis was the observation of a second decay mode for the $Z_{c}(4430)$ with $Z_{c}(4430) \rightarrow J \psi \pi^{ \pm}$, established with a significance of $4.0 \sigma$. The mass and width of the $Z_{c}(4430)$ were fixed in the fit. The measured branching fraction of the $Z_{c}(4430)$ was a factor $\simeq 4$ smaller than for the $Z_{c}(4260)$, presumably due to the smaller phase space. This observation, in addition to the resonant behaviour shown in the Argand diagram in [209] (see below), proves that the $Z_{c}(4430)$ is not a kinematic effect such as induced by a triangle singularity.

To plot the Argand diagrams, the Breit-Wigner shape in the signal regions was replaced by six complex amplitude in consecutive intervals in the invariant mass, that are allowed to float in the fit. Then, the real and imaginary parts of these amplitudes are plotted in a two-dimensions. Figure 32 shows these Argand plots. In the case of $Z_{c}(4200)$ [208], two independent complex $Z$ helicity couplings, $\mathcal{H}_{\lambda}$ for $\lambda=0,+1$, were allowed to float in the fit. Note that parity conservation requires $\mathcal{H}_{-1}=\mathcal{H}_{+1}$. Figure 32 (right) shows only the real and imaginary parts of the $\mathcal{H}_{1}$ amplitude, which is more conclusive than $\mathcal{H}_{0}$. In the case of $Z_{c}(4430)$ [209], the $D$-wave contribution is found to be insignificant when allowed in the fit. Consequently, a pure $S$-wave $Z_{c}$ decay was assumed, implying $\mathcal{H}_{+1}=\mathcal{H}_{0} \equiv \mathcal{A}^{Z}$, for which the real and imaginary parts of the amplitude are shown in Fig. 32 (left). In both cases the phase motion of the amplitude is consistent with that of a Breit-Wigner resonance.

In an additional channel, the decay $B^{0} \rightarrow \chi_{c 1} K^{+} \pi^{-}$was observed to contain two new states called the $Z_{c, 1}(4051)$ and $Z_{c, 2}(4248)$ decaying into $\chi_{c 1} \pi^{-}$, using a data set of $605 \mathrm{fb}^{-1}$ [207]. Figure 31 (second row) shows the $\chi_{c 1} \pi^{\mp}$ invariant mass after applying $K^{*}(892)$ and $K^{*}(1430)$ veto cuts. The $Z_{c, 1}$ and the $Z_{c, 2}$ are clearly visible. The fit shows that the addition of two resonances are preferred by $13.2 \sigma$, and two resonances are preferable to one by $5.7 \sigma$. A fit using Breit-Wigner shapes for both states gives for the $Z_{c, 1}$ a mass of $4051 \pm 14_{-41}^{+20} \mathrm{MeV}$, and a width of $82_{-17-22}^{+21+47} \mathrm{MeV}$, and for the $Z_{c, 2}$ a mass of $4248_{-29-}^{+44+180} \mathrm{MeV}$ and a width of $177_{-39-61}^{+54+316} \mathrm{MeV}$. For the fits, different assumptions for the quantum number assignment combinations of $J_{1,2}=0$ or $J_{1,2}=1$ were tested, however, the $\chi^{2}$ of the fit doesn't change significantly. An important point in these analyses by Belle is the interference (i.e. mixing between the states and interference of the states with the background) was taken into account in the Dalitz fit model. This 

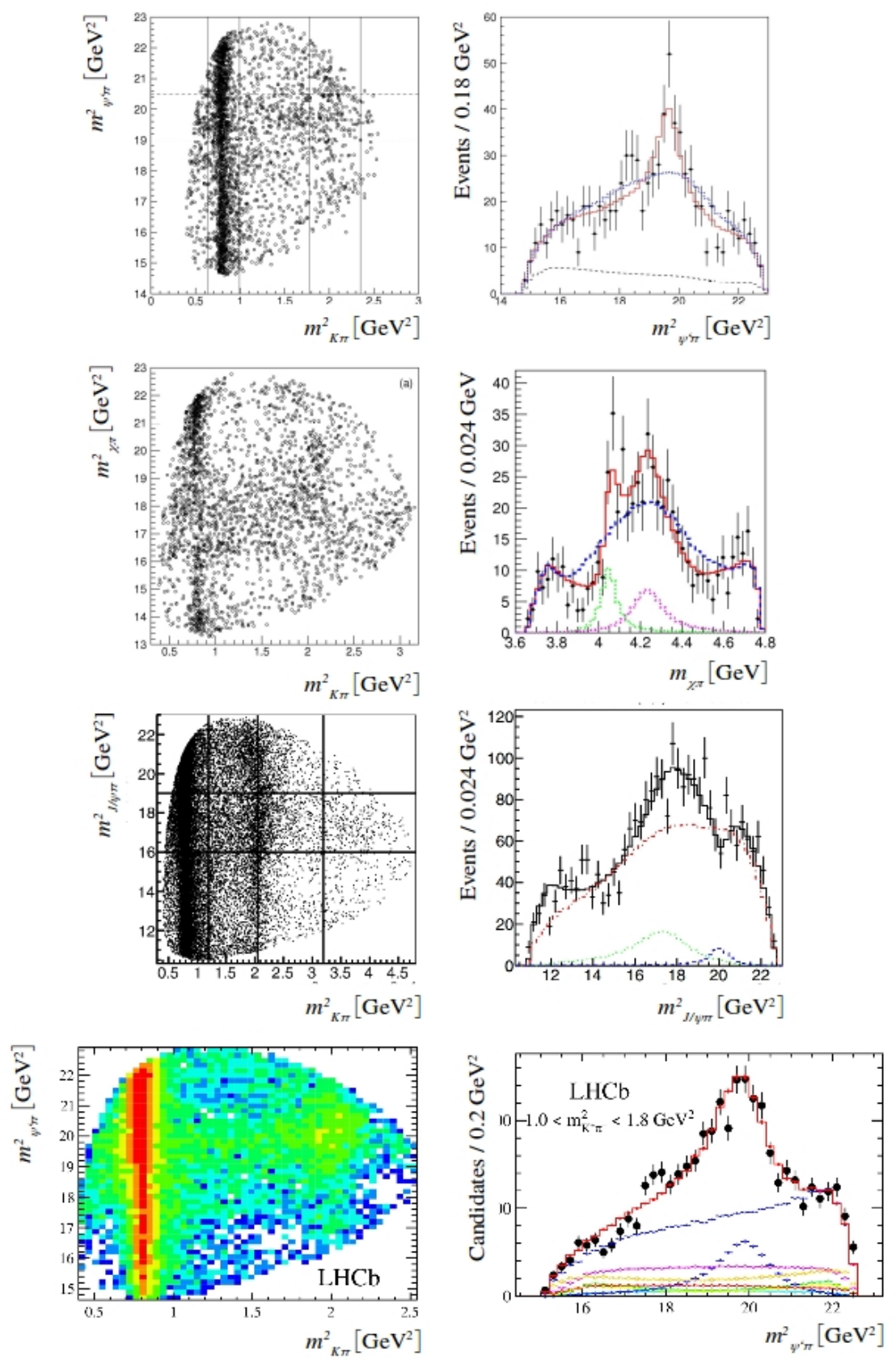

Figure 31: Dalitz plots (left) and mass squared projections for charmonium plus a single charged pion for different final states or experiments. (Top row) $B^{0} \rightarrow \psi^{\prime} K^{ \pm} \pi^{\mp}$ from Belle 205. 206]; $m_{\psi^{\prime} \pi^{+}}^{2}$ is shown with the mass regions around the $K^{* 0}(890)$ and $K^{* 0}(1400)$ being vetoed. (Second row) $B^{0} \rightarrow \chi_{c 1} \pi^{\mp}$ from Belle 207; $m_{\chi_{c 1} \pi^{+}}^{2}$ is shown for a slice in $m^{2}\left(K^{-} \pi^{+}\right)$between 1.0 and $1.75 \mathrm{GeV}^{2}$. The dotted (blue) line shows the fit result without any $\chi_{c 1} \pi^{+}$resonant contributions, and the solid (red) line shows the fit including two resonances. The signal shapes are shown by the dotted (green) line for the $Z_{c, 1}(4051)$, and the dotted (magenta) line for the $Z_{c, 2}(4248)$. (Third row) $B^{0} \rightarrow K^{ \pm} J / \psi \pi^{\mp}$ from Belle $[208] ; m_{J / \psi \pi^{+}}^{2}$ is shown for $2.05<m^{2}\left(K^{-} \pi^{+}\right)<3.2 \mathrm{GeV}^{2}$, the solid (black) line is the result of the total fit, the dashed (red) line is the fit without $Z_{c}$ states, the dotted (green) line shows the $Z_{c}(4200)^{+}$contribution, and the dashed (blue) line shows the $Z_{c}(4430)^{+}$. (Bottom row) $B^{0} \rightarrow \psi^{\prime} K^{ \pm} \pi^{\mp}$ from LHCb 104]. The upper red line indicates to total fit, the upper blue curve indicates the fit without the $Z_{c}(4430)$. The other lines indicate fit contributions from the $Z_{c}$ (4430) (lower blue curve), the $K^{*}(892)$ (dark yellow) the $K \pi S$-wave (magenta), the $K_{s}^{*}(1430)$ (green) the $K^{*}(1680)$ (light yellow) the $K^{*}(1410)$ (light blue) and additional other background sources (lower red curve). For details of the analysis see [209]. 

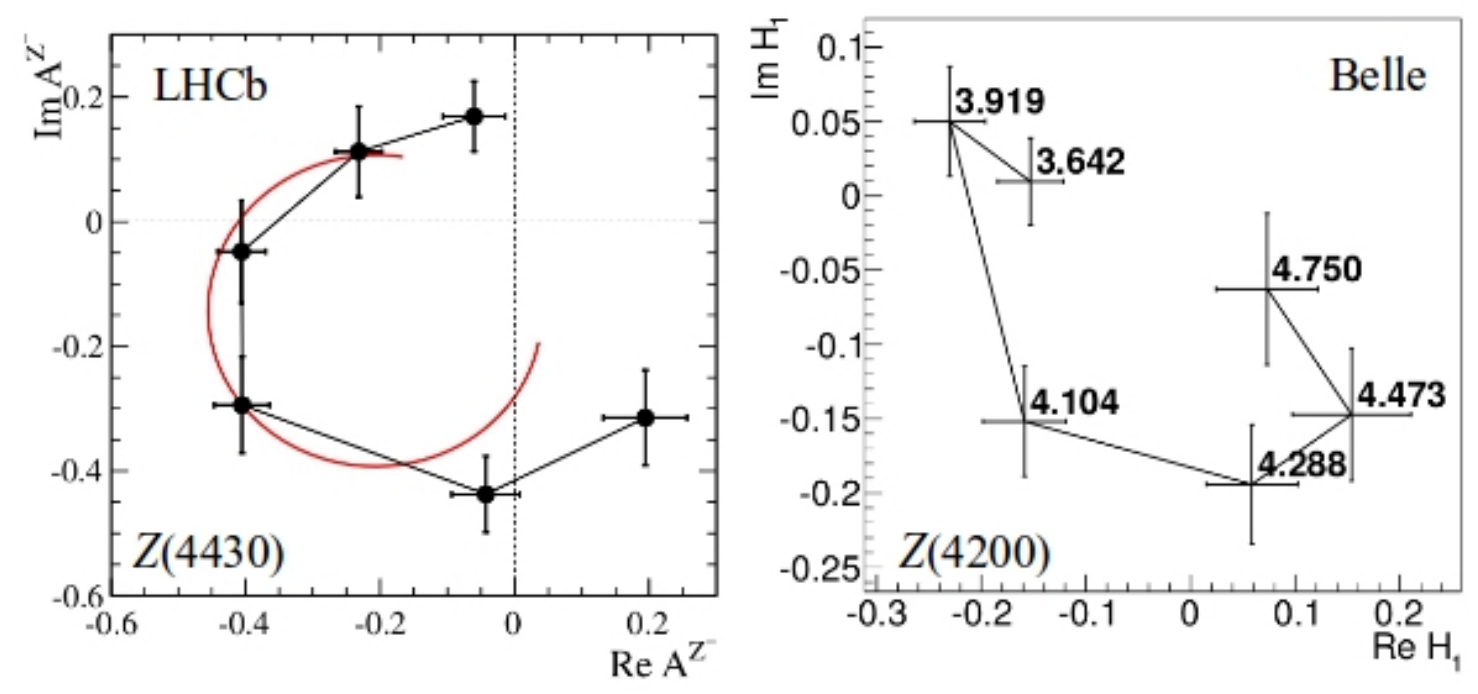

Figure 32: Argand plots for the $Z_{c}$ (4430) (left) from LHCb [209] and the $Z_{c}(4200)$ (right) from Belle [208], proving the resonant nature. For details see text.

can be seen in Fig. 31 in the mass regions $m_{\chi_{c 1} \pi^{ \pm}} \simeq 3.9 \mathrm{GeV}$ and $m_{\chi_{c 1} \pi^{ \pm}} \simeq 4.5 \mathrm{GeV}$, in which the red line (describing the fit with the $Z_{c, 1}$ and the $Z_{c, 2}$ ) falls below the dashed blue line (describing the fit without any $Z$ resonances). It can be seen that in these mass regions the interference is significantly destructive. The $Z_{c, 1}$ and $Z_{c, 2}$ were searched for in an analysis by BABAR [210] in a data set of $429 \mathrm{fb}^{-1}$, but similar to the case of the $Z_{c}(4430)$ no evidence was found and upper limits were assigned.

For a charged state in the charmonium mass regime, the minimal quark content of the $Z_{c}^{+}(4430)$ must be $[c \bar{c} u \bar{d}]$, and thus it cannot be a charmonium state. An interpretation as a $D \bar{D}$ molecule is possible, as the mass is close to the sum of the masses of $D^{*}(2010)$ and $D_{1}(2420)$, both having narrow widths of $96 \mathrm{keV}$ and $20.4 \mathrm{MeV}$, respectively. In the case of the $Z_{c, 1}$, an interpretation as a molecule is also possible, as the sum of the masses $m\left(D^{* 0}\right)+m\left(D^{*+}\right) \simeq 4017 \mathrm{MeV}$ is close to the observed mass of $Z_{c, 1}$ of $4051 \mathrm{MeV}$. However, for the $Z_{c, 2}$, there are no narrow excited $D^{(*)}$ mesons which would fit. The mass and width of the $Z_{2}$ are consistent with the $J^{P}=0^{-} \psi^{\prime} \pi^{ \pm}$state observed by LHCb [209], but a strong decay $0^{-} \rightarrow 1^{+} 0^{-}$is not allowed due to conservation of parity. Thus, there are two different charged states with two different decays, but almost identical mass.

\subsection{Z states of type II in the charmonium mass regime}

Recently, more charged charmonium-like states have been discovered in production mechanisms different from those found in $B$ meson decays. In the following, we will refer to them as $Z$ states of type II. As the first in this class of new states, the $Z_{c}^{+}(3900)$ was observed by BESIII [211] in the decay $Y(4260) \rightarrow Z_{c}^{+}(3900) \pi^{ \pm}$in a data set of $525 \mathrm{pb}^{-1}$ collected at $\sqrt{s}=4.26 \mathrm{GeV}$. The $Z_{c}^{+}(3900)$ was reconstructed in the decay to $J / \psi \pi^{ \pm}$. Fig. 33 (left) shows the observed signal, which has a statistical significance of $>8 \sigma$. From the two charged pions, the one is used which gives the larger $J / \psi \pi^{ \pm}$invariant mass, in order to remove combinatorical background from the pion of the $Y(4260)$ transition to the new state. The measured mass is $3899.0 \pm 3.6 \pm 4.9 \mathrm{MeV}$ and the measured width $46 \pm 10 \pm 20 \mathrm{MeV}$. The observation of the decay $Y \rightarrow Z \pi$ is remarkable, as it provides for the first time a connection between $Z$ states and $Y$ states, possibly pointing to the same interpretation of their nature.

Only a few days later, the state was confirmed by Belle 212 in the same decay channel and also in $Y(4260)$ decays, while in the Belle case the $Y(4260)$ was produced in the ISR process $\Upsilon(n S) \rightarrow$ $\gamma_{I S R} Y(4260)$ (see also Sec. 4.3). The mass of $3894.5 \pm 6.6 \pm 4.5 \mathrm{MeV}$ and the width of $63 \pm 24 \pm 26 \mathrm{MeV}$ are both consistent with the BESIII measurement. Fig. 33 (right) shows the observed signal, which has 

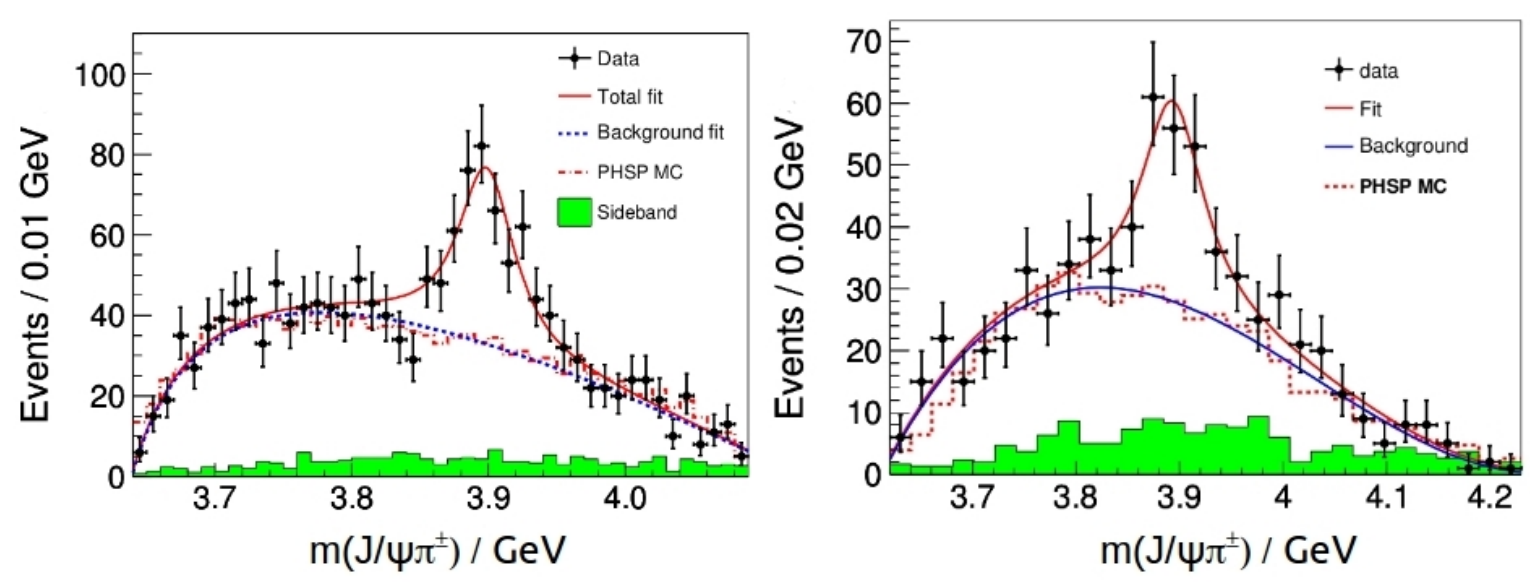

Figure 33: $J / \psi \pi^{\mp}$ invariant mass in Y(4260) decays, indicating the $Z_{c}^{+}(3900)$ signal from BESIII (left) 211 and from Belle (right) 212.

a statistical significance of $>8 \sigma$ in a data set of $967 \mathrm{fb}^{-1}$. Although the yield of produced $Z_{c}(3900)$ is comparable, Fig. 33 (left) corresponds to about 4 weeks of data taking at BESIII, while Fig. 33 (right) corresponds to about 10 years data taking at Belle. Again, as in the case of the $Z_{c}^{+}(4430)$, the state was observed in both positive and negative charged states with about the same yield [211], indicating a doublet.

Concerning the quantum numbers, remarkably the isospin must be $I=1$, (as the isospin of the pion is $I=1$ ), if we assume $I=0$ for the $Y(4260)$. If the heavy meson pair is assumed to be in the $S$-wave, the spin-parity of the state is uniquely determined as $J^{P}=1^{+} . C$-parity $(-1)^{L+S}$ is only defined for neutral particles, thus there can only be a $G$-parity assignment to the $Z_{c}^{+}(3900)$. The $G$-parity $(-1)^{L+S+I}$ with $L=0, S=1$ and $I=1$ thus gives $\mathrm{G}=+$. As $G$-parity should be preserved in strong decays, this assignment, due to the negative $G$-parity of the pion, has the interesting implication that the $Y(4260)$ would have $G=-$. This would be compatible with an $I=0$ isosinglet assignment for the $Y(4260)$, which would imply that there is no charged partner of the $Y(4260)$.

A similar structure to the $Z_{c}(3900)$ was observed in an analysis of CLEO-c data [213] however in data recorded at $\sqrt{s}=4.17 \mathrm{GeV}$. The fitted mass of $3885 \pm 5 \pm 1 \mathrm{MeV}$ and width of $34 \pm 12 \pm 4 \mathrm{MeV}$ of an observed state are consistent with the $Z_{c}(3900)$. The fitted yield is $81 \pm 20$ events, corresponding to a statistical significance of $6.1 \sigma$. However, the lower center-of-mass energy would imply that this state is not produced in $Y(4260)$ decays, but directly via $e^{+} e^{-} \rightarrow Z_{c}^{ \pm} \pi^{\mp}$.

If the $Z_{c}(3900)$ represents a charged partner of the $X(3872)$, one of the important questions is: does it decay not only into a final state with closed charm (i.e. $J / \psi$ ), but also in states with open charm (i.e. $D^{(*)} \bar{D}^{(*)}$ ). In fact, subsequently another type II state was then observed at BESIII [214] in $e^{+} e^{-} \rightarrow \pi^{+}\left(D \bar{D}^{*}\right)^{-}$at $\sqrt{s}=4.26 \mathrm{GeV}$ using a data set of $525 \mathrm{pb}^{-1}$. The mass and width were determined as $3883.9 \pm 1.5 \pm 4.2 \mathrm{MeV}$ and $24.8 \pm 3.3 \pm 11.0 \mathrm{MeV}$, consistent with the mass and the width of the $Z_{c}(3900)$ as measured by BESIII [211]. Fig. 34 (left) shows the $Z_{c}(3885)$ signal from an additional analysis by BESIII 214 with double charmed meson tag, which confirmed the $Z_{c}(3885)$. The angular analysis of the $\pi Z_{c}(3885)$ system leads to a preference for a $J^{P}=1^{+}$assignment (while disfavoring $1^{-}$ or $0^{-}$), which would also be consistent with the $Z_{c}(3900)$ (see above).

The above states obviously are close to the $D^{*} \bar{D}$ threshold. A second class of observed states is close to the $D^{*} \bar{D}^{*}$ threshold. The $Z_{c}(4025)$ was observed at BESIII $\left.\mid 215\right]$ in the reaction $e^{+} e^{-} \rightarrow\left(D^{*} \bar{D}^{*}\right)^{ \pm} \pi^{\mp}$ at $\sqrt{s}=4.26 \mathrm{GeV}$ in a data set of $827 \mathrm{pb}^{-1}$. It was observed by a recoil mass technique with a mass of $4026.3 \pm 2.6 \pm 3.7 \mathrm{MeV}$ and a width of $24.8 \pm 5.6 \pm 7.7 \mathrm{MeV}$. Fig. 34 (center) shows the $\pi^{\mp}$ recoil mass. The $Z_{c}(4025)$ signal is observed with a statistical significance of $\geq 13 \sigma$. 

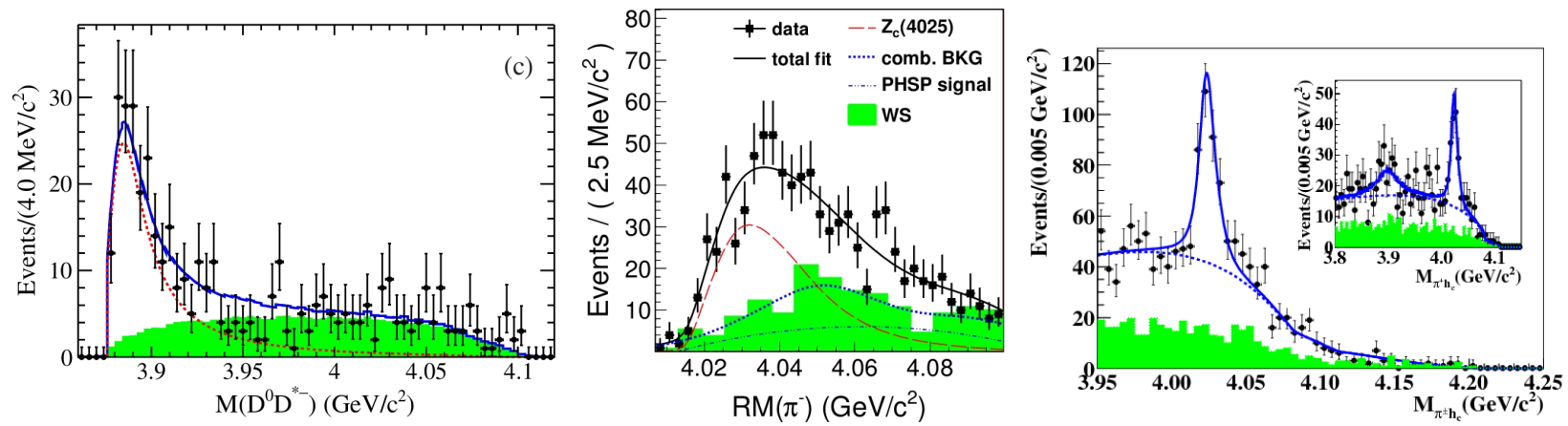

Figure 34: Invariant mass distributions for type II charged states. The solid (green) represents the backgrounds. Left: $D^{0} D^{*-}$ mass at $\sqrt{s}=4.26 \mathrm{GeV}$ showing the signal for the $Z_{c}(3885)$ from BESIII [214] The solid line (blue) shows the combined fit to background and signal, the dotted line (red) shows the signal, and the background is fitted with a phase space probability density function. Center: recoil mass from $\pi^{\mp}$ for $e^{+} e^{-} \rightarrow\left(D^{*} \bar{D}^{*}\right)^{ \pm} \pi^{\mp}$ at $\sqrt{s}=4.26 \mathrm{GeV}$ from BESIII [215]. The black line represents an unbinned maximum likelihood fit. The dashed-line (red) shows the fitted signal contribution of the $Z_{c}(4025)$, the dotted-line (blue) the combinatorial background, and the dash-dotted line (blue) the result of an MC simulation according to a non-resonant phase space distribution. Right: $h_{c} \pi^{ \pm}$invariant mass for $e^{+} e^{-} \rightarrow h_{c} \pi^{ \pm}$at $\sqrt{s}=4.23,4.26$ and $4.36 \mathrm{GeV}$ with the fit for the $Z_{c}$ (4020) from BESIII [216]. The dotted curves show the fitted background. The inset shows the combined fit for the $Z_{c}(4020)$ and the $Z_{c}(3900)$; for the latter no significant signal was observed.

Another type II state, denoted $Z_{c}$ (4020) [216], which may be identical to the $Z_{c}(4025)$, has been observed in a different decay. The data set is comprised of several center-or-mass energies $3.90 \leq \sqrt{s} \leq$ $4.42 \mathrm{GeV}$, i.e. not only $Y(4260)$ decays. It was observed in the $h_{c} \pi^{ \pm}$invariant mass with a mass of $4022.9 \pm 0.8 \pm 2.7 \mathrm{MeV}$ and a width of $7.9 \pm 2.7 \pm 2.6 \mathrm{MeV}$. Fig. 34 (right) shows the fitted signal for a fit of only the $Z_{c}(4020)$ and for a combined fit for the $Z_{c}(4020)$ and the $Z_{c}(3900)$, for which no significant signal was observed. The $h_{c}$ was reconstructed in the decay $h_{c} \rightarrow \gamma \eta_{c}$ with the recontruction of the $\eta_{c}$ in the 16 decays $p \bar{p}, 2\left(\pi^{+} \pi^{-}\right), 2\left(K^{+} K^{-}\right), K^{+} K^{-} \pi^{+} \pi^{-}, p \bar{p} \pi^{+} \pi^{-}, 3\left(\pi^{+} \pi^{-}\right), K^{+} K^{-} 2\left(\pi^{+} \pi^{-}\right), K_{s} K^{ \pm} \pi^{\mp}$, $K_{s} K^{ \pm} \pi^{\mp} \pi^{ \pm} \pi^{\mp}, K^{+} K^{-} \pi^{0}, p \bar{p} \pi^{0}, \pi^{+} \pi^{-} \eta, K^{+} K^{-} \eta, 2\left(\pi^{+} \pi^{-}\right) \eta, \pi^{+} \pi^{-} \pi^{0} \pi^{0}, 2\left(\pi^{+} \pi^{-}\right) \pi^{0} \pi^{0}$. $K_{s}$ mesons are reconstructed from their decay into $\pi \pi^{-}, \pi^{0}$ and $\eta$ mesons are reconstructed from their decay into $\gamma \gamma$.

Interpretation - As the $Z_{c}^{+}(3900)$ is charged, it can neither be a charmonium state nor a hybrid state. The vicinity to the $D^{+} \bar{D}^{0 *}\left(D^{0} \bar{D}^{+*}\right)$ and the $D^{0 *} \bar{D}^{ \pm *}$ thresholds obviously make the states candidates for a charmed meson molecules, similar to the $X(3872)$, Table 11 lists the measured masses, the nearby thresholds, and the mass differences $\Delta m$ between them. There are two important differences with respect to the $X(3872)$. On the one hand (as can be seen in Table 11), in all the cases the masses seem to be higher than the threshold, and thus the "binding" energy would be positive and the state would be a virtual state. For the $X(3872)$, the measured mass is within $\simeq 1 \mathrm{MeV}$ of the threshold and the binding energy is negative, although small. On the other hand, the width of the measured width of the $Z_{c}(3900)$ with $\geq 10 \mathrm{MeV}$ is much larger than in case of the $X(3872)$ with $\leq 1 \mathrm{MeV}$. An interpretation as a tetraquark $[c d]_{\overline{3}}[\overline{c u}]_{3}$ is another option and may explain the isospin $I=1$ e.g. with a direct coupling between the light quarks. In fact, even prior to the observation of the $Z_{c}(3900)$, two nearby tetraquarks with $\mathrm{J}^{P C}=1^{+-}$were predicted $[217]$ with masses of 3.752 and $3.882 \mathrm{GeV}$, respectively. The heavier predicted state may represent the $Z_{c}(3900)$.

These states are presently subject to intensive study in order to clarify their nature. Further data taking at BESIII may largely improve the understanding of these states in the near future. In particular, it may be important to investigate the combined pattern of the three (and possibly more yet to be discovered) states: 
- Are the $Z_{c}(4020)$ and the $Z_{c}(4025)$ identical or different states? One possible way to investigate this question is to assign the quantum numbers using angular distributions.

- Is the $Z_{c}(3900)$ only generated in the decay of the $Y(4260)$, and thus possibly the decay of an exotic state into another exotic state, or also in direct production at center-of-mass energies below?

- In the analysis of the $Z_{c}(4020)$ [216] no significant signal for the $Z_{c}(3900)$ was observed. Is this a result of different quantum numbers, insufficient statistics or does it maybe point to a different nature?

Table 11: Masses, nearby thresholds, and mass differences for charged Z $Z$ states. For all the states, the masses are higher than the thresholds and thus the states may be virtual states.

\begin{tabular}{lcccc|}
\hline State & $m(\mathrm{MeV})$ & Threshold & $\Delta m(\mathrm{MeV})$ & \\
\hline$Z_{c}(3900)$ & $3899.0 \pm 3.6 \pm 4.9$ & $D^{+} \bar{D}^{0 *}$ & +22.4 & 211 \\
$Z_{c}(3900)$ & $3899.0 \pm 3.6 \pm 4.9$ & $D^{0} \bar{D}^{+*}$ & +23.9 & 211 \\
$Z_{c}(3900)$ & $3894.5 \pm 6.6 \pm 4.5$ & $D^{+} \bar{D}^{0 *}$ & +17.9 & 212 \\
$Z_{c}(3900)$ & $3894.5 \pm 6.6 \pm 4.5$ & $D^{0} \bar{D}^{+*}$ & +19.4 & 212 \\
$Z_{c}(3900)$ & $3885 \pm 5 \pm 1$ & $D^{+} \bar{D}^{0 *}$ & +8.4 & $\underline{\underline{\underline{213}}}$ \\
$Z_{c}(3900)$ & $3885 \pm 5 \pm 1 \mathrm{MeV}$ & $D^{0} \bar{D}^{+*}$ & +9.9 & 213 \\
$Z_{c}(3885)$ & $3883.9 \pm 1.5 \pm 4.2$ & $D^{+} \bar{D}^{0 *}$ & +7.4 & $\mid 218$ \\
$Z_{c}(3885)$ & $3883.9 \pm 1.5 \pm 4.2$ & $D^{0} \bar{D}^{+*}$ & +8.8 & 218 \\
$Z_{c}(4020)$ & $4022.9 \pm 0.8 \pm 2.7$ & $D^{0 *} \bar{D}^{ \pm *}$ & +5.6 & 216 \\
$Z_{c}(4025)$ & $4026.3 \pm 2.6 \pm 3.7$ & $D^{0 *} \bar{D}^{ \pm *}$ & +9.0 & 215 \\
\hline
\end{tabular}

Table 12: Masses and widths in comparison for the charged and neutral $Z$ states observed at BESIII.

\begin{tabular}{|c|c|c|c|c|}
\hline State & $m(\mathrm{MeV})$ & Width $(\mathrm{MeV})$ & Decay & \\
\hline$Z_{c}(3900)^{+}$ & $3899.0 \pm 3.6 \pm 4.9$ & $46 \pm 10 \pm 20$ & $J / \psi \pi^{+}$ & 211 \\
\hline$Z_{c}(3900)^{0}$ & $3894.8 \pm 2.3 \pm 2.7$ & $29.6 \pm 8.2 \pm 8.2$ & $J / \psi \pi^{0}$ & 219 \\
\hline$Z_{c}(3885)^{+}$ & $3883.9 \pm 1.5 \pm 4.2$ & $24.8 \pm 3.3 \pm 1.0$ & $\left(D D^{*}\right)^{+}$ & 215 \\
\hline$Z_{c}(3885)^{0}$ & $3885.7_{-5.7}^{+4.3} \pm 8.4$ & $35_{-12}^{+11} \pm 15$ & $\left(D D^{*}\right)^{0}$ & $\mid \overline{220}$ \\
\hline$Z_{c}(4020)^{+}$ & $4022.9 \pm 0.8 \pm 2.7$ & $7.9 \pm 2.7 \pm 2.6$ & $h_{c} \pi^{+}$ & 216 \\
\hline$Z_{c}(4020)^{0}$ & $4023.8 \pm 2.2 \pm 3.8$ & Fixed to 7.9 & $h_{c} \pi^{0}$ & 221 \\
\hline$Z_{c}(4025)^{+}$ & $4026.3 \pm 2.6 \pm 3.7$ & $24.8 \pm 5.6 \pm 7.7$ & $\left(D^{*} D^{*}\right)^{+}$ & 215 \\
\hline$Z_{c}(4025)^{0}$ & $4025.5_{-4.7}^{+2.0} \pm 3.1$ & $23.0 \pm 6.0 \pm 1.0$ & $\left(D^{*} D^{*}\right)^{0}$ & 222 \\
\hline
\end{tabular}

Soon after the discovery of the charged $Z_{c}^{+}(3900)$ [211] the neutral partner state, decaying into $J / \psi \pi^{0}$ was discovered by BESIII [219]. On the one hand, this observation may lead to the tempting conclusion of isospin triplets, i.e. that the $Z^{0, \pm}$ may carry isospin $I=1$. This conclusion is supported by the observation, that the ratio of the cross sections of $e^{+} e^{-} \rightarrow Z^{0} \pi^{0}$ and $e^{+} e^{-} \rightarrow Z^{ \pm} \pi^{\mp}$ is found to be close to unity for the $Z_{c}(3885)$ [220], the $Z_{c}(4020)$ [221] and the $Z_{c}(4025)$ [222]. On the other hand, the observation of both charged and neutral states points to different 4-quark contents: $[c \bar{c} u \bar{d}]$ or $[c \bar{c} d \bar{u}]$ for the charged $Z,[c \bar{c} u \bar{u}]$ or $[c \bar{c} d \bar{d}]$ for the neutral $Z$. In such a case, the masses may be different. Meanwhile, for all four observed charged $Z$ states [211] [218] [216] [215], corresponding neutral partners were identified [219] [220] [221] [222]. Table 12 shows the comparison of the masses and the widths of corresponding charged and neutral $Z$ states. 
If, in fact, if $Z$ states of type II are isospin triplets, the interesting question would be: where is the $I=0$ partner? Let us consider the $Z_{c}(3900)$ as an example. The $X(3872)$ is a tempting candidate, even though $\simeq 10 \mathrm{MeV}$ below (as the $Z$ states are all slightly above threshold) and significantly more narrow $(\leq 1.2 \mathrm{MeV}$ instead of a few tens $\mathrm{MeV})$. In the case of the $Z_{c}^{0, \pm}(3900)$, from the decay to $J / \psi$ $\pi$ and assuming $L=0$ due to the vicinity of the threshold, the quantum number assignment would be $J^{P}=1^{+}$, identical to the $J^{P}$ of the $X(3872)$. However, the $X(3872)$ carries $C=+1$. Under the above assumptions, the $Z^{0}(3900)$ may only carry $C=-1$. Note that in the tetraquark interpretation, the $Z(3900)^{0}$ will carry a charge conjugation quantum number in any case, as with a $[c \bar{c} q \bar{q}]$ the $Z$ would be its own antiparticle. The only question is, if the content is $[c \bar{c} d \bar{d}]$ or $[c \bar{c} u \bar{u}]$, however not changing the quantum number assignment.

An interesting observation is, that the branching fractions of decays into open charm meson pairs are large compared to branching fracions into charmonium, which may indicate molecular contributions in the wave function.

- Assuming, that the $Z_{c}(3900)$ and the $Z_{c}(3885)$ are the same state, the ratio of the branching fractions of the decay into $\left(D D^{*}\right)^{ \pm}$and into $J / \psi \pi^{+}$is $6.2 \pm 1.1 \pm 2.7[218]$.

- Assuming, that the $Z_{c}(4020)$ and the $Z_{c}(4025)$ are the same state, the ratio of the branching fractions of the decay into $\left(D D^{*}\right)^{ \pm}$and into $h_{c} \pi^{+}$is $12 \pm 5[223$.

We emphasize that this is very different from $Y(4260)$ decays, for which no open charm decay has ever been found (see Sec. 4.3 . However, there is strong evidence that all the $Z$ states of type II are produced in decays of the $Y(4260)$. If $Y$ states and $Z$ states of type II are of the same nature, the decay pattern seems to be quite different. The ratio can be compared to the other cases:

- For the $X(3872)$, the ratio [12] is

$$
\frac{\mathcal{B}\left(X(3872) \rightarrow D^{0} D^{0 *}\right)}{\mathcal{B}\left(X(3872) \rightarrow J \psi \pi^{+} \pi^{-}\right)}=\frac{>24 \%}{>2.6 \%}
$$

- For the conventional charmonium states $\psi(3770)$, the ratios of decays into $D^{*} \bar{D}^{*}$ and $J / \psi \pi^{+} \pi^{-}$ is very large with $482 \pm 84[12$. In a simplified point of view, it seems that open charm decays of $Z$ states are blocked by a yet unknown mechanism.

Summarizing, we find the following differences for the two classes of $Z$ states. $Z_{c}$ states of type I are observed in $B$ meson decays, not obviously related to any nearby threshold, have large widths (partially $\geq 100 \mathrm{MeV}$ or more) and are so far only observed as charged states. $Z_{c}$ states of type II are observed in $e^{+} e^{-}$and ISR production, are found within a few $\mathrm{MeV}$ of a nearby threshold (and, perhaps as a hint to the nature of these states, even a few $\mathrm{MeV}$ above threshold), are narrow with a maximum width of a few tens $\mathrm{MeV}$, and are observed as charged and neutral states (within the errors of the measurement with degenerate masses and identical widths), and thus probably representing isospin triplets. No common member of type I and type II has been found yet. Thus, at present, it must be assumed that these are two different classes of $Z$ states. As a striking fact, the quantum numbers are $1^{+}$for any state of type I and any state of type II, whereever tested experimentally.

\subsection{Z $Z$ states in the bottomonium mass regime}

In $\Upsilon(5 \mathrm{~S})$ decays, Belle observed two new states with masses of $m=9898.3 \pm 1.1_{-1.1}^{+1.0} \mathrm{MeV}$ and $m=10259.8 \pm 0.6_{-1.0}^{+1.4} \mathrm{MeV}$, respectively [16]. These new states are widely accepted to represent the conventional bottomonium states $h_{b}\left(1^{1} P_{1}, 1^{+-}\right)$and $h_{b}^{\prime}\left(2^{1} P_{1}, 1^{+-}\right)$, as their masses were found 
Table 13: Measured masses and width of the charge $Z_{b}$ and $Z_{b}^{\prime}$ states.

\begin{tabular}{lccccc}
\hline & $\Upsilon(1 S) \pi^{+} \pi^{-}$ & $\Upsilon(1 S)(2 S) \pi^{+} \pi^{-}$ & $\Upsilon(1 S)(3 S) \pi^{+} \pi^{-}$ & $h_{b}(1 P) \pi^{+} \pi^{-}$ & $h_{b}(2 P) \pi^{+} \pi^{-}$ \\
\hline$m\left(\mathrm{Z}_{b}(10610)\right)(\mathrm{MeV})$ & $10611 \pm 4 \pm 3$ & $10609 \pm 2 \pm 3$ & $10608 \pm 2 \pm 3$ & $10605 \pm 2_{-1}^{+3}$ & $10599_{-3-4}^{+6+5}$ \\
$\Gamma\left(\mathrm{Z}_{b}(10610)\right)(\mathrm{MeV})$ & $22.3 \pm 7.7_{-4.0}^{+3.0}$ & $24.2 \pm 3.1_{-3.0}^{+2.0}$ & $17.6 \pm 3.0 \pm 3.0$ & $11.4_{-3.9-1.2}^{+4.5+2.1}$ & $13.0_{-8}^{+100+9}$ \\
$m\left(\mathrm{Z}_{b}(10650)\right)(\mathrm{MeV})$ & $10657 \pm 6 \pm 3$ & $10651 \pm 2 \pm 3$ & $10652 \pm 1 \pm 2$ & $10654 \pm 3_{-2}^{+1}$ & $10651_{-3-2}^{+2+3}$ \\
$\Gamma\left(\mathrm{Z}_{b}(10650)\right)(\mathrm{MeV})$ & $16.3 \pm 9.8_{-2.0}^{+6.0}$ & $13.3 \pm 3.3_{-3.0}^{+4.0}$ & $8.4 \pm 2.0 \pm 2.0$ & $20.9_{-4.7-5.7}^{+5.4}$ & $19 \pm 7_{-7}^{+11}$ \\
\hline
\end{tabular}

within $2.7 \mathrm{MeV}$ and $1.2 \mathrm{MeV}$ of relativistic potential model predictions [225]. In a second step, the observation of the $h_{b}(1 P)$ and the $h_{b}(2 P)$ also enabled the study of their specific production mechanism in $\Upsilon(5 S)$ decays, i.e. are they produced according to phase space or are there any intermediate resonances. Surprisingly, both the $h_{b}(1 P) \pi^{+} \pi^{-}$and $h_{b}(2 P) \pi^{+} \pi^{-}$final states contain a large fraction of $h_{b}(n \mathrm{P}) \pi^{ \pm}$resonances. In the analysis [224], instead of directly plotting the $h_{b}(n \mathrm{P}) \pi^{ \pm}$mass, the recoil mass off of the bachelor charged pion is plotted, because its relatively low momentum leads to excellent mass resolution. In addition to the $h_{b}(n \mathrm{P}) \pi^{ \pm}$systems, the $\Upsilon(n S) \pi^{ \pm}$systems were investigated. In fact, all five systems show two intermediate resonances [224], which similar to the $Z$ states in charmonium (Sec. 4.4), were given the names $Z_{b}\left(\right.$ or $\left.Z_{b}(10610)\right)$ and $Z_{b}^{\prime}\left(\right.$ or $Z_{b}(10650)$ ). Figure 35 shows the recoil mass for $\Upsilon(1 S) \pi^{ \pm}, \Upsilon(2 S) \pi^{ \pm}$and $\Upsilon(3 S) \pi^{ \pm}$. As these two resonances are charged, they cannot be bottomonium states. Fits were performed using two Breit-Wigner shapes with different masses and widths. For $\Upsilon(5 S) \rightarrow \Upsilon(n S) \pi^{+} \pi^{-}$an $S$-wave Breit-Wigner shape wass taken, as the $\Upsilon$ states have the same quantum numbers. For $\Upsilon(5 S) \rightarrow h_{b}(n P) \pi^{+} \pi^{-}$a $P$-wave Breit-Wigner shape was taken because of $\Delta S=1$ for the $b \bar{b}$ transition. Phases $\phi_{i}$ were included into the fit functions by $\exp \left(i \phi_{i}\right)$ terms for the different signals $i$. Table 13 shows the fitted masses, widths and statistical significances. As the observation of the same two resonances is made in five different final states, the total significance is very high. Note that, as a result from fitting with two Breit-Wigner shapes with a relative phase, the result is that the phase in $\Upsilon(n S)$ and $h_{b}(n P)$ final states seems to be shifted by $180^{\circ}$. Interestingly, the $Z_{b}$ is very close to the $B^{0 *} \bar{B}^{ \pm}$threshold and the $Z_{b}^{\prime}$ to the $B^{0 *} \bar{B}^{* \pm}$ threshold. Mass differences with respect to the thresholds are only $+2.6 \mathrm{MeV}$ and $+2.0 \mathrm{MeV}$, respectively. Note the surprising fact, that both mass differences are positive and thus indicate no binding energy in the system, although $\Delta m$ and the errors in the mass determination of $2.0 \mathrm{MeV}$ and $1.5 \mathrm{MeV}$ are of the same order of magnitude, and thus some caution is still advised before drawing a conclusion. If this behavior is confirmed, the states would be very similar to the $Z$ states of type II in the charmonium mass regime.

An angular analysis was performed by Belle as well. In particular $(a)$ the angle between the charged pion $\pi_{1}$ and the $e^{+}$from the $\Upsilon$ decay and $(b)$ the angle between the plane $\left(\pi_{i}, e^{+}\right)$and the plane $\left(\pi_{1}, \pi_{2}\right)$
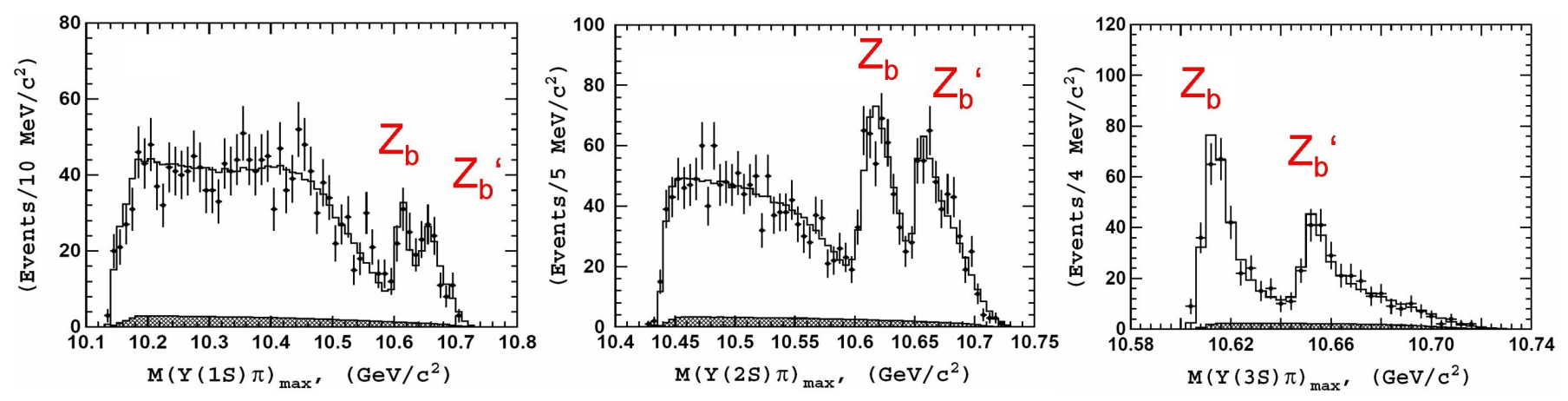

Figure 35: Charged pion recoil mass for $\Upsilon(5 S) \rightarrow \Upsilon(1 S) \pi^{+} \pi^{-}$(left), $\Upsilon(5 S) \rightarrow \Upsilon(2 S) \pi^{+} \pi^{-}$(center) and $\Upsilon(5 S) \rightarrow \Upsilon(3 S) \pi^{+} \pi^{-}$(left) from Belle 224 . The $Z_{b}$ and $Z_{b}^{\prime}$ states are labelled. 
turned out to be useful. For example, in case of $J^{P}=1^{+}$both distributions are approximately flat, while for $1^{-}, 2^{+}$and $2^{-}$they indicate parabolic shapes. Note that the quantum numbers $0^{+}$and $0^{-}$are forbidden by parity conservation. All distributions turned out to be consistent with $J^{P}=1^{+}$, while all other quantum numbers were disfavoured at typically $\geq 3 \sigma$ level. Thus, it is likely that the $Z_{b}$ and the $Z_{b}^{\prime}$ carry the same spin and parity as the $X(3872)$. Note that $C$-parity is only defined for neutral states, therefore only $G$-parity could be assigned to the $Z$ states, but has not been investigated yet.

There are numerous attempts to explain the $Z_{b}$ states e.g. coupled channel effects [226], a cusp effect [227] or tetraquarks [217] [228]. A particular attempt was made [229] to explain the states along with the anomalous observations in $h_{b}$ production. The ansatz is to interpret the new resonances as $B^{0 *} \bar{B}^{ \pm}$and $B^{0 *} \bar{B}^{* \pm}$ molecular states, and to form $1^{+}$states based upon the quantum number from the angular distribution tests (see above). The $Z_{b}$ and $Z_{b}^{\prime}$ states are constructed as orthogonal states in the limit of large $m_{b}$ quark mass. In this limit, their heavy quark spin structure should be the same as of the following pairs,

$$
\begin{aligned}
\left|Z_{b}\right\rangle & =\frac{1}{\sqrt{2}}\left(0_{b \bar{b}}^{-} \otimes 1_{q \bar{q}}^{-}+1_{b \bar{b}}^{-} \otimes 0_{q \bar{q}}^{-}\right) \\
\left|Z_{b}^{\prime}\right\rangle & =\frac{1}{\sqrt{2}}\left(0_{b \bar{b}}^{-} \otimes 1_{q \bar{q}}^{-}-1_{b \bar{b}}^{-} \otimes 0_{q \bar{q}}^{-}\right) .
\end{aligned}
$$

Here $0^{-}$and $1^{-}$stand for para- and ortho-states with negative parity, and both $Z_{b}$ and $Z_{b}^{\prime}$ are assumed to have $J^{P}=1^{+}$. The above representation in its Fierz transformed form implies that the decays of the type $Z_{b}^{\prime} \rightarrow B^{*} \bar{B}^{*}$ are forbidden, despite being allowed by phase space. The other consequences of this model are:

1. It would be expected, that the two states are degenerate in the large $m_{b}$ limit. Therefore the widths should be equal, and this might explain why the $h_{b} \pi^{+} \pi^{-}$final state is not suppressed relative to $\Upsilon(n S) \pi^{+} \pi^{-}$.

2. The relative phase of the coupling of these two resonances to the ortho-bottomonium (i.e. the $b \bar{b}$ part) is opposite to that for the para-bottomonium. This would explain the relative phase difference of $180^{\circ}$.

3. It also would explain the similarity of the mass differences of $m\left(Z_{b}^{\prime}\right)-m\left(Z_{b}\right) \simeq 50 \mathrm{MeV}$ and $m\left(B^{*}\right)-$ $m(B) \simeq 46 \mathrm{MeV}$.

For further details see 229]. These features can equally well be explained in the tetraquark interpretation of the two $Z_{b}$ states, irrespective of whether they are the decay products of the $\Upsilon(5 S)$, or of the $Y_{b}(10890)$, as discussed in the section on theoretical models.

The hidden $b \bar{b}$ state $Y_{b}(10890)$ with $J^{\mathrm{P}}=1^{--}$was discovered by Belle in 2007 [230] in the process $e^{+} e^{-} \rightarrow Y_{b}(10890) \rightarrow(\Upsilon(1 S), \Upsilon(2 S), \Upsilon(3 S)) \pi^{+} \pi^{-}$just above the $\Upsilon(5 S)$. More on this state later.

Similar to the case of the type II $Z_{c}$ states (see Sec. 4.5) it was found, that decays to meson pairs seem to dominate, i.e. $\mathcal{B}\left(Z_{b}(10610) \rightarrow B \bar{B}^{*}+\right.$ c.c. $) / \mathcal{B}\left(Z_{b}(10610) \rightarrow\right.$ bottomonium $)=4.76 \pm 0.64 \pm 0.75$ and $\mathcal{B}\left(Z_{b}(10650) \rightarrow B^{*} \bar{B}^{*}\right) / \mathcal{B}\left(Z_{b}(10650) \rightarrow\right.$ bottomonium $)=2.40 \pm 0.44 \pm 0.50$ [231]. This may indicate molecular contributions in the wave function. For the $Z_{b}(10610)$, a neutral partner has been observed, pointing to an isospin triplet similar to the type II $Z_{c}$ states (see Sec. 4.5). Principally, in case of sufficiently precise experimental mass resolution, this would allow tests ofs different quark contents, e.g. $[b \bar{b} u \bar{d}]$ or $[b \bar{b} d \bar{u}]$ for the charged $Z_{b}$ and $[b \bar{b} u \bar{u}]$ or $[b \bar{b} d \bar{d}]$ for the neutral $Z_{b}$. However, so far the measured masses are compatible within the errors, with $10609 \pm 4 \pm 4 \mathrm{MeV}$ for the neutral $Z_{b}[232]$ and $10607.2 \pm 2.0$ for the charged $Z_{b}[27]$. 


\subsection{Another curious system: $J / \psi \phi$ resonances}

Charmonium states are composed of $c \bar{c}$ quarks. The charged states described above decaying into a $J / \psi \pi^{ \pm}$or $\psi^{\prime} \pi^{ \pm}$cannot be composed of only $c \bar{c}$. Resonant states decaying into $J / \psi \phi$ could be charmonium states or have a more exotic quark content. In 2009 the CDF collaboration found $58 \pm 10$ signal events in the channel $B^{-} \rightarrow J / \psi \phi K^{-}$[233]. The $J / \psi \phi$ invariant mass spectrum showed a spike near $4140 \mathrm{MeV}$, close to the threshold of about $4116 \mathrm{MeV}$, with a very narrow width of about $15 \mathrm{MeV}$.

Feynman diagrams for the $B^{-}$decay into $J / \psi \phi K^{-}$are shown in Fig. 36. One possibility is that the decay reflects simply the well known process $B^{-} \rightarrow J / \psi K^{*-}$ with $K^{*-} \rightarrow \phi K^{-}$instead of $K^{-} \pi^{0}$ or $K^{0} \pi^{-}$. Another, implied by the narrowness of the observed state, is that the $c \bar{c} s \bar{s}$ produced in the decay form an object called the $X(4140)$ plus an additional $K^{-}$. Yet another process involves the production of an exotic $Z$ like state that decays into $J / \psi K^{-}$; here the $u \bar{u}$ produced in the decay must transform into a $\phi$, which makes this process very unlikely.

The CDF data can be seen in Fig. 37(a). The fit is to an $S$-wave relativistic Breit-Wigner convolved with the experimental mass resolution for the peak near $4140 \mathrm{MeV}$ plus a three-body phase space background. The significance of the peak is $5 \sigma$. Another peak near $4.3 \mathrm{GeV}$ is also present in the fit and gives a $3.1 \sigma$ significance.

Other experiments followed. D0 observed a similar structure at $4159 \mathrm{MeV}$ with a somewhat larger width of $19.9 \mathrm{MeV}$ [234]. (All mass and width values are listed in Table 14.) The second structure was seen but the statistics were insufficient to determine either the mass or width [Fig. 37(b)]. CMS followed with clear observations of both structures with the lower mass peak at $4148 \mathrm{MeV}$ and a width of $28 \mathrm{MeV}$ [235]; they claimed evidence for but not observation of the higher mass structure [Fig. 37(c)]. There were also experiments that did not observe the peak near $4140 \mathrm{MeV}$ including Babar [Fig. 37(d)] [236], LHCb with a $0.37 \mathrm{pb}^{-1}$ sample [Fig. 37(e)] [237), and Belle [Fig. 37(f)] [238]. While the $X(4140)$ state remained controversial, the data from the different experiments seems to have a significant enhancement near $4300 \mathrm{MeV}$, although this was not emphasized, and indeed no statistically significant observation in any experiment had been made.

In $2016 \mathrm{LHCb}$ performed a full amplitude analysis of this final state [241]. Since the final state is three-body, a Dalitz plot can be constructed and is shown in Fig. 38 . $\left.95\right|^{13}$ Note that there are structures evident in both the $J / \psi \phi$ and $\phi K^{-}$masses. The amplitude formalism used is very similar to that used for analysis of the pentaquark states described in Section 3.2 [11]. For the pentaquark states the first decay mode that was analyzed was $\Lambda_{b}^{0} \rightarrow J / \psi K^{-} p$, a similar three-body final state to the one

\footnotetext{
${ }^{13}$ Dalitz plots for three spinless particles in the final state reveal directly the structure of the matrix element. For non-zero spin they are also instructive.
}
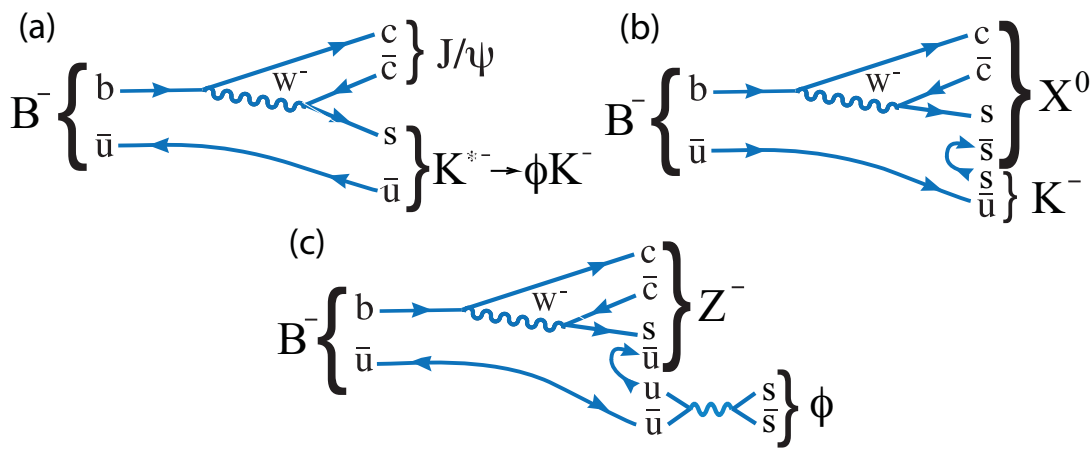

Figure 36: Diagrams for $B^{-} \rightarrow J / \psi \phi K^{-}$proceeding via three possible intermediate states. a) The $\phi K^{-}$ states are produced by an intermediate $K^{*-}$. (b) Neutral exotic states are produced by the merger of the $c \bar{c} s \bar{s}$ quarks. (c) There is an exotic $Z^{-}$resonance formed from $c \bar{c} s \bar{u}$ quarks. For this to happen the $u \bar{u}$ must make a $\phi$ meson, which dominantly has an $s \bar{s}$ wavefunction. 
(a)

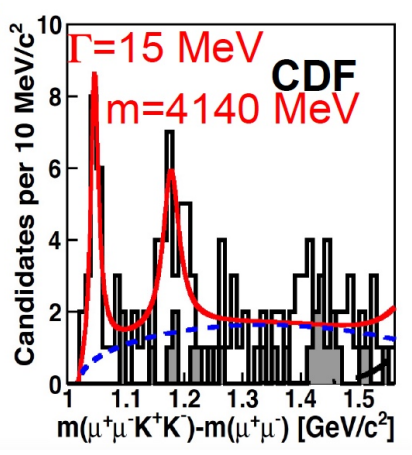

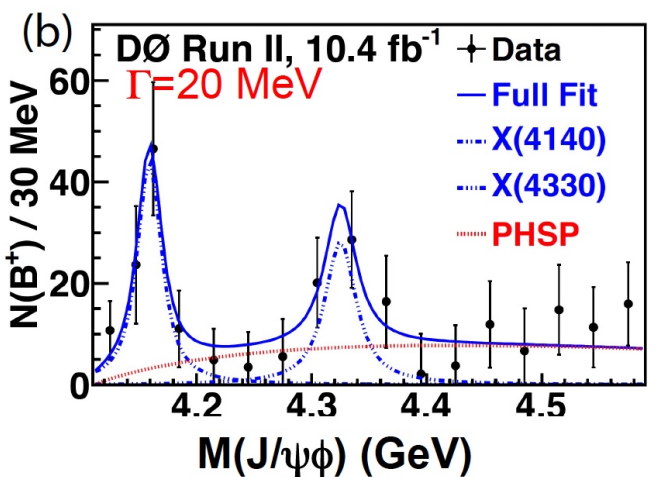
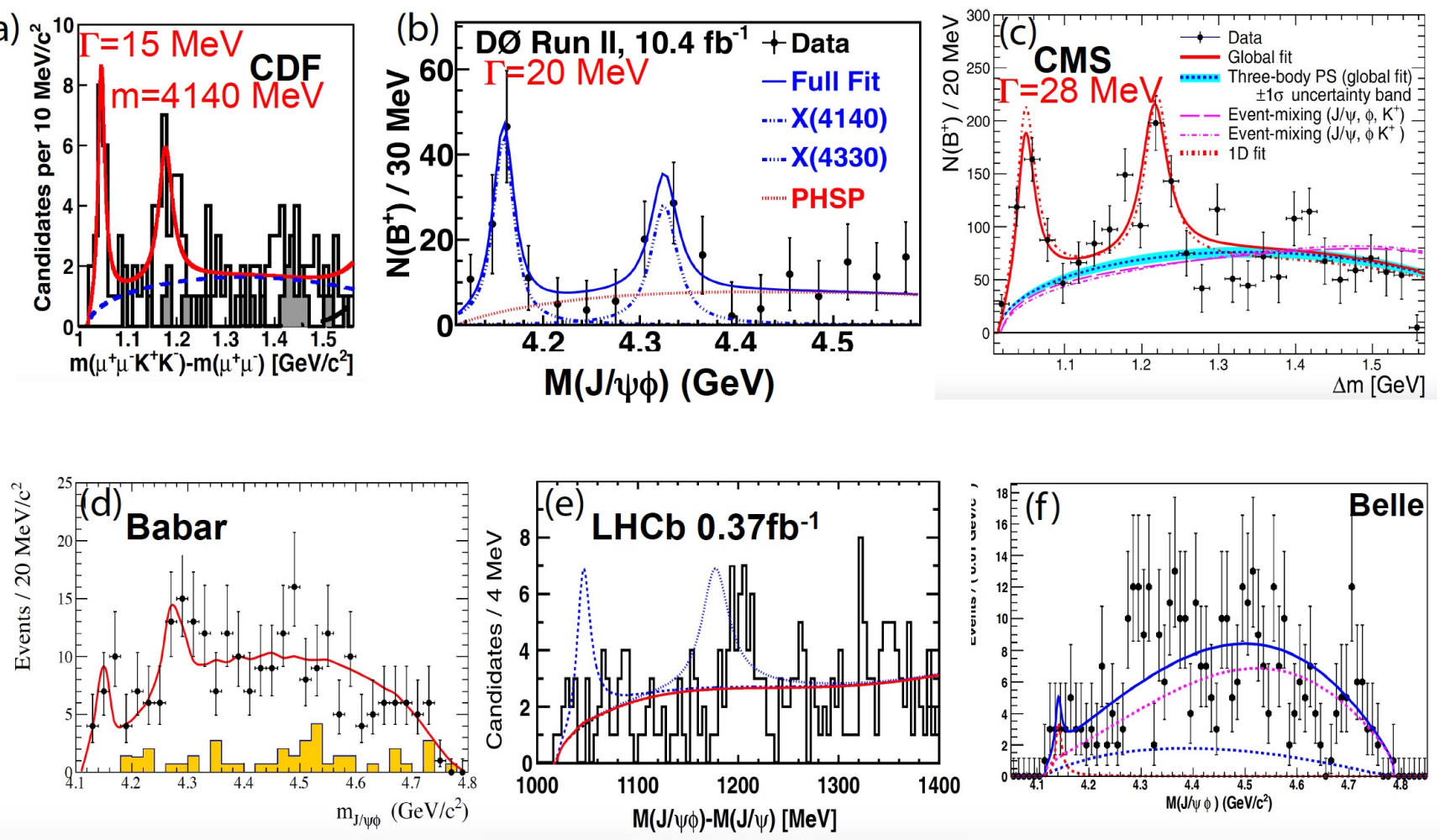

Figure 37: Experimentally determined $J / \psi \phi$ mass spectra for $B^{-} \rightarrow J / \psi \phi K^{-}$decay. From (a) CDF [233], (b) D0 [234], (c) CMS [235], (d) BABAR [236], (e) LHCb [237, 239], and (f) Belle [238]. The graphs in the variable $\Delta m$ can be compared to the others by adding the $J / \psi$ mass of $3097 \mathrm{MeV}$.

considered here with the $J / \psi$ decay into muons being a common feature. Of course the spins of the particles are different and the amplitudes must reflect this, giving different decay angular distributions for the different resonant final states. Here all three amplitudes shown in Fig. 36 are allowed to interfere. (It turns out the one describing the possible $Z^{-}$has no effect on the results and is used only to set part of the systematic uncertainty.)

While resonant structures in most analyses have relied on seeing Breit-Wigner shaped peaks in mass spectrum, the absence of such evidence does not mean that several resonances are not present,

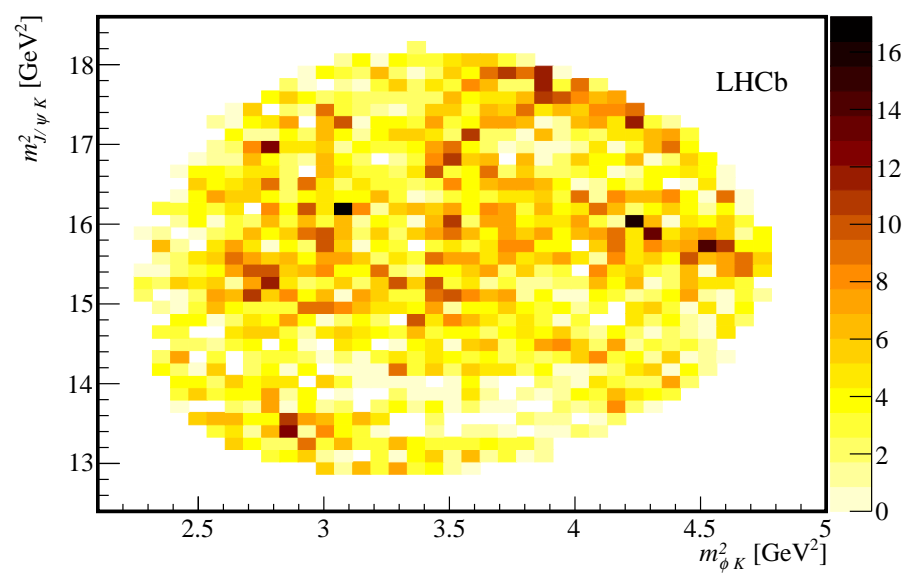

Figure 38: Dalitz plot for $B^{-} \rightarrow J / \psi \phi K^{-}$decays, background subtracted and efficiency corrected (from 241]). 
Table 14: History of $X(4140)$ and $X(4274)$ observations in $B^{-} \rightarrow J / \psi \phi K^{-}$prior to 2016 . The term "fix" means the values were not taken from the data but fixed to previous measurements. The number of standard deviation significances $(\sigma)$ are given when quoted by the experiments. The u.l. in the LHCb row for $X(4140)$ indicates an upper limit that is in contradiction with the CDF result by $2.4 \sigma$.

\begin{tabular}{|c|c|c|c|c|c|c|}
\hline \multicolumn{7}{|c|}{$X(4140)$} \\
\hline Year & Exp. & $\mathrm{fb}^{-1}$ & $\# B^{-}$ & Mass $(\mathrm{MeV})$ & $\Gamma(\mathrm{MeV})$ & $\sigma$ \\
\hline 2008 & CDF $[233]$ & 2.7 & $58 \pm 10$ & $4143.0 \pm 2.9 \pm 1.2$ & $11.7_{-5.0}^{+8.3} \pm 3.7$ & 3.8 \\
\hline 2009 & Belle $\overline{238}$ & 840 & $325 \pm 21$ & 4143 fix & 11.7 fix & 1.9 \\
\hline 2011 & CDF 240 & 6.0 & $115 \pm 12$ & $4143.4_{-3.0}^{+2.9} \pm 0.6$ & $15.3_{-6.1}^{+10.4} \pm 2.5$ & 5.0 \\
\hline 2011 & $\mathrm{LHCb} 237$ & 0.37 & $346 \pm 20$ & 4143.4 fix & 15.3 fix & u.l. \\
\hline 2013 & CMS 235 & 5.2 & $2480 \pm 160$ & $4148.0 \pm 2.4 \pm 6.3$ & $28.0_{-11}^{+15} \pm 19$ & 5.0 \\
\hline 2013 & D0 234 & 10.4 & $215 \pm 37$ & $4159.0 \pm 4.3 \pm 6.6$ & $19.9 \pm 12.6_{-8.0}^{+1.0}$ & 3.1 \\
\hline 2014 & BABAR 236 & 422 & $189 \pm 14$ & 4143.4 fix & 15.3 fix & 1.6 \\
\hline \multicolumn{7}{|c|}{$X(4274)$} \\
\hline Year & Exp. & $\mathrm{fb}^{-1}$ & $\# B^{-}$ & Mass $(\mathrm{MeV})$ & $\Gamma(\mathrm{MeV})$ & $\sigma$ \\
\hline 2008 & CDF [233] & 2.7 & $58 \pm 10$ & & & \\
\hline 2009 & Belle 238 & 840 & $325 \pm 21$ & & & \\
\hline 2011 & CDF $\overline{240}$ & 6.0 & $115 \pm 12$ & $4274.4_{-6.7}^{+8.4} \pm 1.9$ & $32.3_{-15.3}^{+21.9} \pm 7.6$ & 3.1 \\
\hline 2011 & $\mathrm{LHCb}[237]$ & 0.37 & $346 \pm 20$ & 4274.4 fix & 32.3 fix & \\
\hline 2013 & CMS 235 & 5.2 & $2480 \pm 160$ & $4313.8 \pm 5.3 \pm 7.3$ & $38_{-15}^{+30} \pm 16$ & \\
\hline 2013 & D0 234 & 10.4 & $215 \pm 37$ & 4328.5 & 30 fix & \\
\hline 2014 & BABAR 236 & 422 & $189 \pm 14$ & 4274.4 fix & 32.2 fix & 1.2 \\
\hline
\end{tabular}

merely that they can be wide and therefore washed out. Conversely, there have been arguments that seeing such peaks may be evidence of the rescattering of intermediate particles rather than resonance structures. We will return to this discussion later.

The efficiency corrected and background subtracted projections of the Dalitz plot are shown in Fig. 39. The $\phi K^{-}$mass spectra seems devoid of resonant activity, while the $J / \psi \phi$ shows evidence of structures. LHCb first tried to fit the data with only $K^{*-}$ contributions. While the $\phi K^{-}$mass distribution was adequately described, the peaks in the $J / \psi \phi$ distribution could not be satisfactorily reproduced (see Fig. 40). There is always some art in Dalitz analyses in choosing the number of such resonances to use. Generally those that are not significant are dropped. ${ }^{14}$ The amplitude analysis performed by $\mathrm{LHCb}$ reveals the presence of several resonant $K^{*-} \rightarrow \phi K^{-}$states as well as four $J / \psi \phi$ structures.

The projections of the fit are shown in Fig. 41. The amplitude analysis allows not only their masses and widths to be determined, but also their $J^{P}$. Table 15 gives the five putative resonant structures found in the fit, while Table 16 lists the $K^{*-}$ resonances. Fig. 42 shows the fit projections in terms of the angular variables. (While a complete discussion of the $K^{*}$ states found here is outside of the scope of this review, these results add importantly to our knowledge of these resonances.)

Finding four states was quite unexpected and surprising. The lowest mass state near $4140 \mathrm{MeV}$ is consistent with the mass of the state claimed by CDF, but its width is substantially higher. In fact, a narrow mass peak is not present in the $\mathrm{LHCb}$ data, showing inconsistency with the findings of CDF, D0 and CMS. This state is revealed in the $\mathrm{LHCb}$ data only through the amplitude analysis. The state at $4274 \mathrm{MeV}$ was put on a firm foundation and the data are consistent among the experiments. The higher mass states were not seen by other experiments; this is perhaps an acceptance issue.

${ }^{14}$ At least one author has given some thought to these matters 242 . 

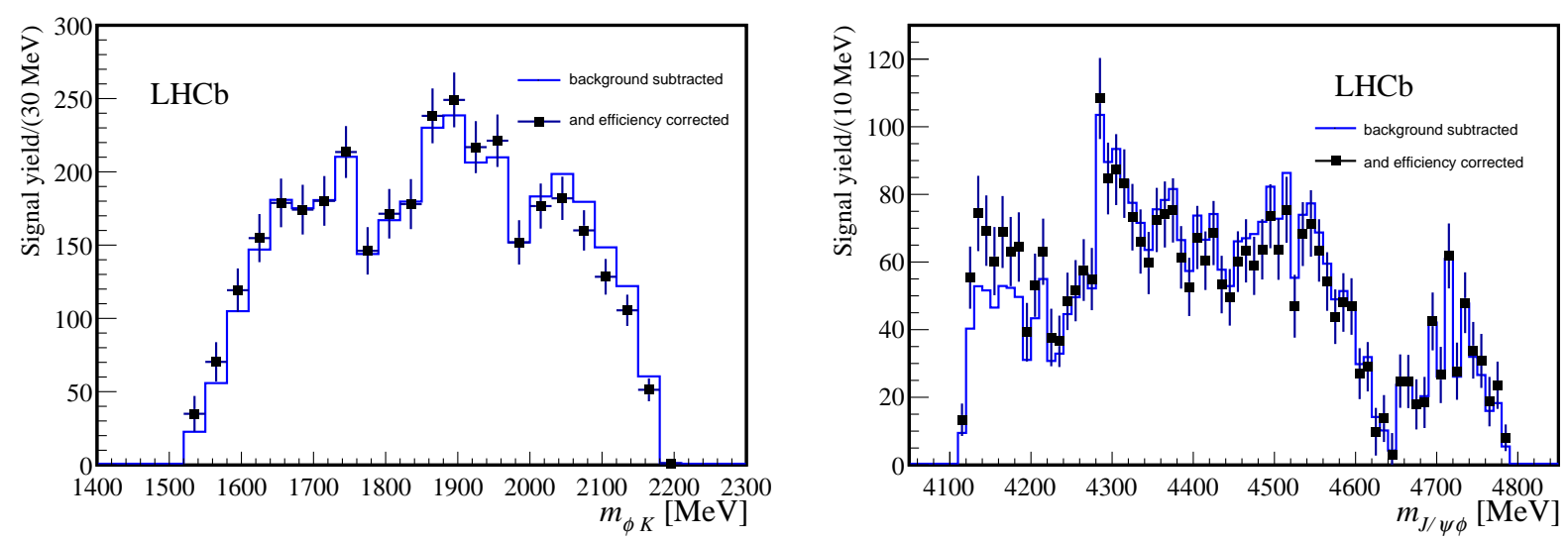

Figure 39: Invariant mass distributions of $\phi K^{-}$(left) and $J / \psi \phi$ (right), background subtracted and efficiency corrected (from [241]). The data are shown once with background subtraction only and then again efficiency corrected, so as to emphasize the relatively small nature of the efficiency corrections.

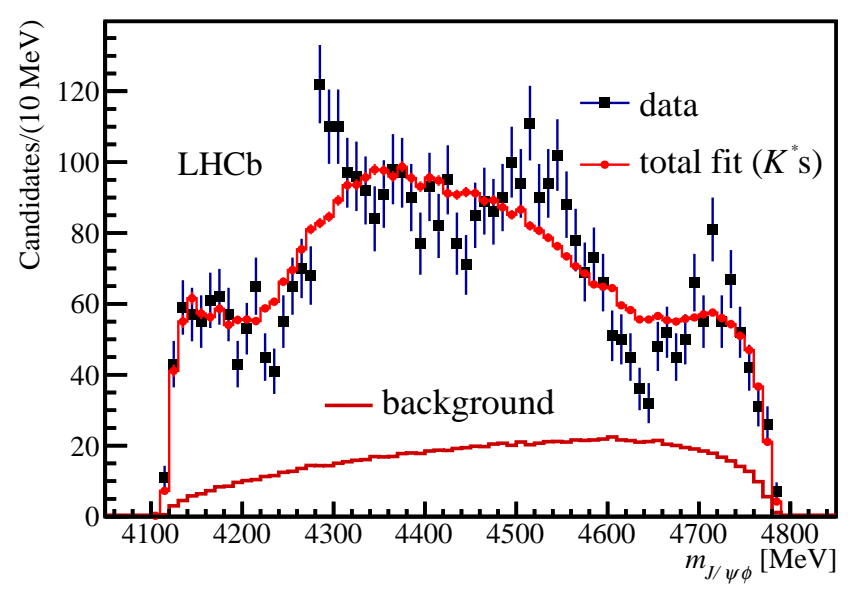

Figure 40: Best fit of $J / \psi \phi$ mass distribution using only $K^{*-}$ resonances (from [241]).

Another possibility is that these are not resonance states but a manifestation of rescattering. The basic idea is that a basic process such as $B^{-} \rightarrow D_{s}^{*-} D_{s}^{+} K^{-}$occurs and the $D_{s}^{*-} D_{s}^{+}$rescatter into $J / \psi \phi$. Since the mass of the $D_{s}^{*-}$ plus $D_{s}^{+}$of $4080 \mathrm{MeV}$ is just below $4140 \mathrm{MeV}$, such a cusp could cause behavior similar to that of a resonance 243,244 . LHCb did fit their data to a variant of the Swanson model for the $4140 \mathrm{MeV}$ state [243]. The fit was marginally better than for a Breit-Wigner resonance. The other states were found not be describable by this model.

Prior to the LHCb analysis there had been other proposals explaining the $X(4140)$ including a molecular state [245 253], or a tetraquark state [254 258], hadrocharmonium [43], or a hybrid state [259,260]. It remains to be seen which of these can explain the data. We note, however, that molecular states or re-scattering models cannot account for the $1^{+}$nature of the $X(4274)$.

It is difficult to understand the observed $\left(1^{+}, 1^{+}, 0^{+}, 0^{+}\right)$pattern seen here and is suggestive that not all of them have the same nature. Many explanations of these states have appeared subsequent to the LHCb publication. One interesting suggestion starts with taking the underlying structure of these states as $c \bar{c} s \bar{s}$ tetraquarks, that these can be further broken down into $c s$ and $\overline{c s}$ diquarks, and then they assume that the spin-spin interactions inside the diquark are dominant [261]. They predict the masses of states with this tetraquark content depending on the $J^{P C}$ of the resonance. They suggest that the state near $4274 \mathrm{MeV}$ is not $1^{+}$but the superposition of a $0^{++}$state with a $2^{++}$state. Further analysis will be needed to see if this is required by the data. In the hadrocharmonium model [43], the $X(4140)$ can be described as as a $J / \psi \phi$ state and would correctly give the observed $1^{+}$in $S$-wave. Although the 

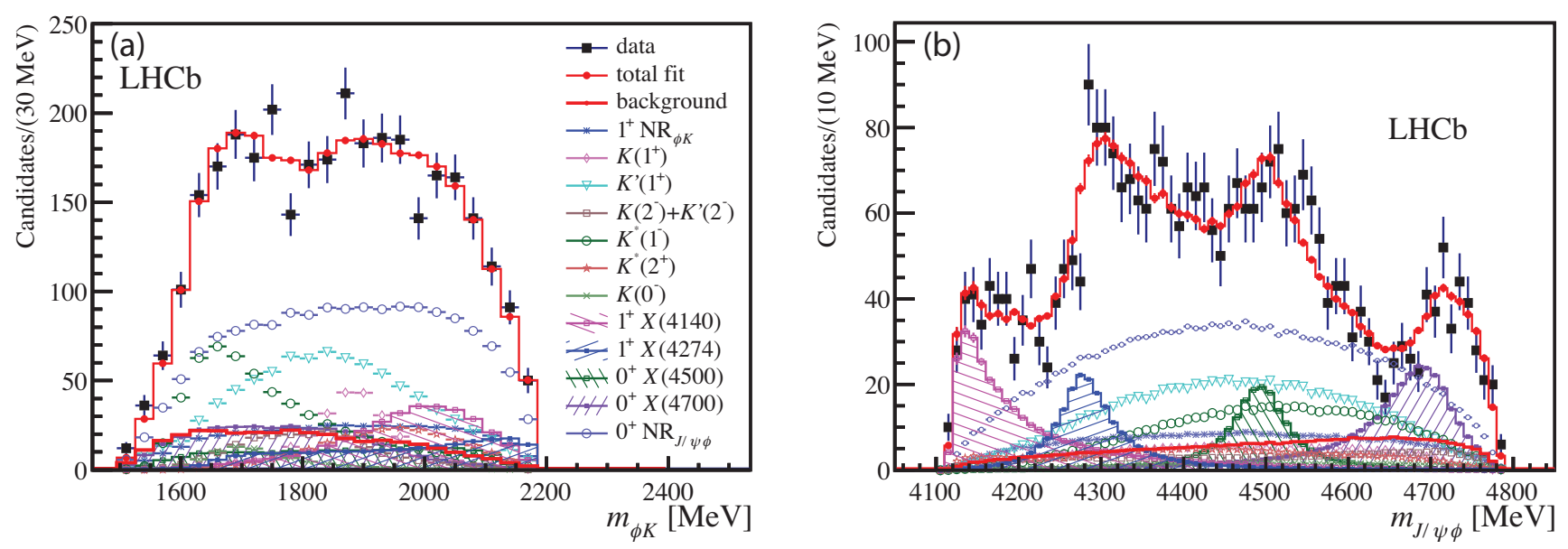

Figure 41: Fit projections of the mass distributions for (a) $\phi K^{-}$and (b) $J / \psi \phi$ (right). The separate resonance contributions are also shown (from [241]).

Table 15: Properties of resonant $J / \psi \phi$ states found in the LHCb amplitude analysis, and the nonresonant (NR) fraction.

\begin{tabular}{lccccc}
\hline Particle & $J^{P}$ & $\sigma$ & $\begin{array}{c}\text { Mass } \\
(\mathrm{MeV})\end{array}$ & $\begin{array}{c}\text { Width } \\
(\mathrm{MeV})\end{array}$ & \multicolumn{1}{c}{$\begin{array}{c}\text { Fit } \\
\text { Fraction(\%) }\end{array}$} \\
\hline$X(4140)$ & $1^{+}$ & 8.4 & $4146 \pm 4.5_{-2.8}^{+4.6}$ & $83 \pm 21_{-14}^{+21}$ & $13.0 \pm 3.2_{-2.0}^{+4.8}$ \\
$X(4274)$ & $1^{+}$ & 6.0 & $4273.3 \pm 8.3_{-3.6}^{+17.2}$ & $56 \pm 11_{-11}^{+8}$ & $7.1 \pm 2.5_{-2.4}^{+3.5}$ \\
$X(4500)$ & $0^{+}$ & 5.6 & $4506 \pm 11_{-15}^{+12}$ & $92 \pm 21_{-14}^{+21}$ & $6.6 \pm 2.4_{-2.3}^{+3.5}$ \\
$X(4700)$ & $0^{+}$ & 5.6 & $4704 \pm 10_{-24}^{+14}$ & $120 \pm 31_{-33}^{+42}$ & $12 \pm 5_{-5}^{+9}$ \\
$\mathrm{NR}$ & $0^{+}$ & 6.4 & & & $46 \pm 11_{-21}^{+11}$ \\
\hline
\end{tabular}

$s \bar{s}$ component does not resemble a light quark cloud (see Sec. 5 below), the mass of the $\phi$ is still within the range of the model. However, it would be a virtual state, as the mass is $\simeq 20 \mathrm{MeV}$ above the $J / \psi \phi$ threshold at $4116 \mathrm{MeV}$.

Another state near $4350 \mathrm{MeV}$ was found by Belle in $\gamma \gamma \rightarrow \phi J / \psi$ collisions with $3.2 \sigma$ significance. [262]. It needs confirmation. Clearly it is not seen in the $B^{-}$decay investigated by LHCb.

\section{Theoretical models for tetraquarks}

The exotic particles discussed in the preceding sections have either a hidden $c \bar{c}$ or a $b \bar{b}$ pair in their Fockspace. No exotic hadron with a single $c$ or a single $b$ quark has been found yet. No doubly-charged exotic hadron has been seen so far either. However, the nonet of the lightest scalar mesons in the Particle Data Group, called $\sigma$ or $f_{0}(500), \kappa(800), a_{0}(980)$ and $f_{0}(980)$, have been argued to be tetraquark candidates due to their inverted mass hierarchy compared to the light pseudoscalar and vector hadrons, with the $(I=1) f_{0}(980)$ heavier than the $I=1 / 2 \kappa(800)$, and the isosinglet $f_{0}(500)$ being the lightest 263.264. The dynamics of the light scalar mesons is influenced by the infrared sector of QCD. In particular, instanton effects play a crucial role and this was recognized already in the early QCD epoch 265 269]. We shall not discuss the light scalar nonet here and will restrict our discussion to the charmonium-like and bottomonium-like exotics in which, due to the heavy quark constituents, instanton-induced effects are anticipated to be small. Several explicit kinematic and dynamical mechanisms have been devised to 

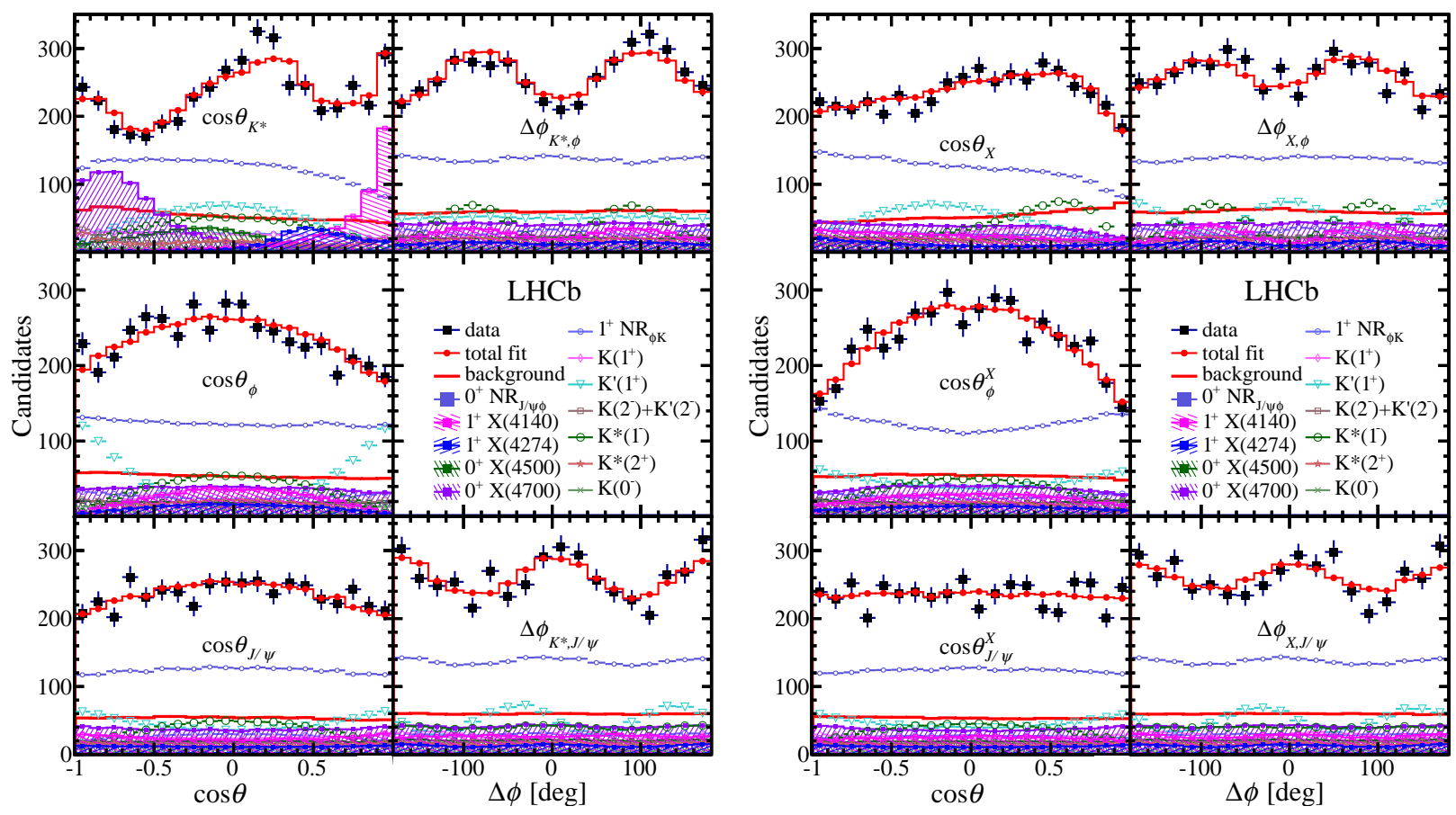

Figure 42: Fit projections of the angular distributions from the $K^{*-}$ decay sequence (left) and $J / \psi \phi$ chain (right). The separate resonance contributions are also shown (from [241]).

work out the spectroscopy of the quarkonia-like exotics, which we have already mentioned earlier. They go by the names: cusps, hadroquarkonia, hybrids, hadron molecules, and compact diquarks. Theoretical details are, however, still rather sketchy, and an underlying organizational principle is either lacking or not yet properly formulated. In particular, production cross-sections of the multiquark hadrons are not yet calcuable. Despite this, some characteristic features specific to each of the theoretical framework can be defined and will be discussed below.

\subsection{Tetraquarks as cusps}

The cusp approach is used to explain the origin of the charged states $Z_{c}(3900)\left[D \bar{D}^{*}\right], Z_{c}(4025)\left[D^{*} \bar{D}^{*}\right]$, $Z_{b}(1610)\left[B \bar{B}^{*}\right]$, and $Z_{b}(10650)\left[B^{*} \bar{B}^{*}\right]$, as their masses lie just above the indicated thresholds. For tetraquarks [243, 270], it is assumed that threshold re-scatterings are enough to describe the data, and as such there is no need for poles in the scattering matrix. Discussed long ago by Wigner [182] in the context of the non-relativistic two-body scattering theory, and resucitated more recently by Törnqvist [31] in an attempt to understand the low-lying scalar meson $q \bar{q}$ nonet, and in a broader sweep by Bugg 271] interpreting the resonances as synchronized artefacts, this effect has to do with the behavior of the scattering cross-sections $\sigma(E)$ near thresholds, say $E=E_{0}$. As one approaches $E_{0}$ from above or below, the cross-section remains finite but the slope $d \sigma(E) / d E \rightarrow-\infty$, indicating a discontinuity, which can result in a cusp, as $\sigma(E)$ is continued below $E_{0}$. This can be illustrated by mapping the two-particle scattering amplitude to a two-point function (self-energy), thereby relating the opening channel singularity to the self-energy threshold singularity. The imaginary part of the selfenergy $\operatorname{Im} \Pi(s)$ is zero for $\sqrt{s}$ below the threshold, and it turns on rapidly once the threshold is crossed 15 The resulting enhancements by cusps can mimic genuine S-matrix poles (resonances). However, the two can be distinguished by studying the phase motion of the amplitudes. Representing a resonance

\footnotetext{
${ }^{15}$ Here $E$ and $\sqrt{s}$ are used interchangeably.
} 
Table 16: Properties of resonant $K^{*-}$ states found in the LHCb amplitude analysis, and the nonresonant (NR) fraction taken as a $1^{+}$contribution. Predicted masses are from Godfrey and Isgur [54] The fraction of longitudinal and transverse polarizations are available in [241].

\begin{tabular}{lcccccc}
\hline Particle & $n^{2 S+1} L_{J}$ & $\sigma$ & $\begin{array}{c}\text { Mass } \\
(\mathrm{MeV})\end{array}$ & $\begin{array}{c}\text { Measured } \\
\text { Width } \\
(\mathrm{MeV})\end{array}$ & $\begin{array}{c}\text { Fit } \\
\text { Fraction(\%) }\end{array}$ & $\begin{array}{c}\text { Predicted } \\
\text { Mass } \\
(\mathrm{MeV})\end{array}$ \\
\hline$K\left(1^{+}\right)$ & $2^{1} P_{1}$ & 7.6 & $1793 \pm 59_{-101}^{+153}$ & $365 \pm 157_{-215}^{+157}$ & $12 \pm 10_{-6}^{+17}$ & 1900 \\
$K^{\prime}\left(1^{+}\right)$ & $2^{3} P_{1}$ & 1.9 & $1968 \pm 65_{-172}^{+70}$ & $396 \pm 170_{-178}^{+174}$ & $23 \pm 20_{-29}^{+31}$ & 1930 \\
$\mathrm{NR}$ & & & & & $42 \pm 8_{-9}^{+5}$ & \\
$K\left(2^{-}\right)$ & $1^{1} D_{2}$ & 5.0 & $1777 \pm 35_{-77}^{+122}$ & $217 \pm 116_{-154}^{+221}$ & $11 \pm 3_{-5}^{+2 \dagger}$ & 1780 \\
$K^{\prime}\left(2^{-}\right)$ & $1^{1} D_{2}$ & 3.0 & $1853 \pm 27_{-35}^{+18}$ & $167 \pm 58_{-72}^{+83}$ & $\dagger$ Shared with $K\left(2^{-}\right)$ & 1810 \\
$K^{*}\left(1^{-}\right)$ & $1^{3} D_{2}$ & 8.5 & $1722 \pm 20_{-109}^{+} 30$ & $354 \pm 75_{-181}^{+140}$ & $6.7 \pm 1.9_{-3.9}^{+3.2}$ & 1780 \\
$K *\left(2^{+}\right)$ & $2^{3} P_{2}$ & 5.4 & $2073 \pm 94_{-240}^{+245}$ & $678 \pm 311_{-559}^{+1153}$ & $2.9 \pm 0.8_{-0.7}^{+1.7}$ & 1940 \\
$K\left(0^{-}\right)$ & $3^{1} S_{0}$ & 3.5 & $1874 \pm 43_{-115}^{+}$ & $168 \pm 90_{-104}^{+280}$ & $2.6 \pm 1.1_{-1.8}^{+2.3}$ & 2020 \\
\hline
\end{tabular}

by a Breit-Wigner amplitude, $f(s)=\frac{\Gamma}{2} /(M-\sqrt{s}-i \Gamma / 2)$, the magnitude and phase of this amplitude vary with $\sqrt{s}$, according to a circular trajectory in the Argand diagram. Cusps, on the other hand, have characteristically different dependence on $\sqrt{s}$. In terms of the variable $z=c\left(m_{A}+m_{B}-\sqrt{s}\right)$, where $A$ and $B$ are the intermediate states and $c$ is a normalizing constant, one can show that the imaginary part of a cusp amplitude is zero for positive $z$ (i.e., below threshold) and turns on rapidly as the threshold is crossed, reflecting essentially the function $\operatorname{erf} c(\sqrt{z})$, which governs $\operatorname{Im}(\Pi(s))$. This phase motion differs, in principle, from that of a genuine Breit-Wigner. In the experimental analysis of some of the exotic mesons this phase motion is not measured, and in those cases a cusp-interpretation remains a logical, though by no means a unique, option. However, in at least three cases, $Z_{c}(4430)$, $Z_{c}(4200)$ and $P_{c}(4450)$, data analysis produced Argand diagram; the first two are shown in Fig. 32 and the third in Fig. 13, in complete agreement with the characteristic Breit-Wigner motion for a genuine resonance.

\subsection{Tetraquarks as hadroquarkonia}

This mechanism is motivated by analogy with the hydrogen atom. In the hadroquarkonium model, a $Q \bar{Q}$ $(Q=c, b)$ pair forms the hard core surrounded by light matter (light $q \bar{q}$ in the case of tetraquarks and $q q q$ for pentaquarks), with the two systems bound by the QCD analog of a residual van der Walls type force. For example, the hadrocharmonium core may consist of the $J / \psi, \psi^{\prime}$ or $\chi_{c}$, and the light $q \bar{q}$ degrees of freedom can be combined to accommodate the observed hadrons [43]. The effective Hamiltonian for the interaction can be written using the QCD multipole expansion $H_{\text {eff }}=-1 / 2 \alpha^{\left(\psi_{1} \psi_{2}\right)} E_{i}^{a} E_{i}^{a}$, where $E_{i}^{a}$ is a chromoelectric field, and $\alpha^{\left(\psi_{1} \psi_{2}\right)}$ is the chromopolarizability, which can be measured from the dipionic transitions involving two heavy quarkonia $Q \bar{Q}$ states $\psi_{1} \rightarrow \psi_{2} \pi^{+} \pi^{-}$. Decays into heavy flavor mesons in this picture are suppressed, as this requires the splitting of the $Q \bar{Q}$ core by means of the soft gluons, present in the cloud. A variation on this theme is that the hard core quarkonium could be in a color-adjoint representation [272], in which case the light degrees of freedom are also a color-octet to form an overall singlet. Hadroquarkonium models have conceptual problems: if the binding force is weak, the question is why the system remains stable for long enough a time to be identified as a distinct state. If the force is strong, it is not clear why the $Q \bar{Q}$ core and the light degrees of freedom don't rearrange themselves as a pair of heavy mesons $\left(D \bar{D}^{*}, B \bar{B}^{*}\right.$ etc.). While this does seem to happen in the decays of the $Z_{b}(10610)$ and $Z_{b}(10650)$, generally this is not the case, as discussed in the previous section. This would have suppressed the appearance of the states $\left(J / \psi, h_{c}\right) \pi \pi$ in their decays, which in 
fact, are in many cases the discovery modes of such exotic multiquark states.

\subsection{Tetraquarks as hybrids}

The hybrid models for exotic hadrons are based on the QCD-inspired flux-tubes, which predict exotic $J^{\text {PC }}$ states of both the light and heavy quarks $[273$. Hybrids are hadrons formed from the valence quarks and gluons, for example, consisting of $Q \bar{Q} g$, following the color algebra

$$
3_{c} \otimes \overline{3}_{c} \otimes 8_{c}=\left(8_{c} \oplus 1_{c}\right) \otimes 8_{c}=27_{c} \oplus \overline{10} \overline{0}_{c} \oplus 10_{c} \oplus 8_{c} \oplus 8_{c} \oplus 8_{c} \oplus 1_{c},
$$

resulting in a color-singet hybrid hadron. States dominated by gluons form glueballs, which are firm predictions of QCD, but have proven to be so far elusive experimentally. Current lattice-QCD computations [274] suggest that non-perturbative gluons, the object of interest in constructing the hybrids, are quasiparticles having $J^{P C}=1^{+-}$with an excitation energy of approximately $1 \mathrm{GeV}$. This would put the lightest charmonium hybrid multiplets at around $4200 \mathrm{MeV}$. Extensive studies of such hybrids have been carried out on the lattice by the Hadron Spectrum Collaboration [275], though for a heavy pion mass, $m_{\pi} \sim 400 \mathrm{MeV}$ and at fixed lattice spacing. More recently, simulations are also undertaken at a lower mass, and the results for $m_{\pi} \sim 240 \mathrm{MeV}$ from Ref. [59] are shown in Fig. 11. Several states in this computation are identified as charmonium hybrid multiplets, having the quantum numbers $J^{P C}=0^{-+}, 1^{--}, 2^{-+}, 1^{-+}$, with their masses $(M)$ estimated to lie in the range $M-M_{\eta_{c}} \simeq 1200-1400$ $\mathrm{MeV}$. Very much along the same lines, but much earlier, a hybrid interpretation was advanced for the $J^{\mathrm{PC}}=1^{--}$state $Y(4260)$, which has a small $e^{+} e^{-}$annihilation cross section $47,276,277$. In the meanwhile, hybrids have been offered as templates for other exotic hadrons as well [278, 279]. They have been put on firmer theoretical footings in the framework of effective field theories [280]. Despite all these theoretical advances, which are impressive and may eventually provide reliable quantitative predictions, an unambiguous hybrid candidate has yet to be identified in the current experiments. Advances in lattice QCD techniques, enabling a firm phenomenological profile of the glueballs and the $Q \bar{Q} g$ hybrids, and dedicated experiments, such as the GlueX [281] and PANDA [282], may change this picture dramatically.

\subsection{Tetraquarks as hadron molecules}

This very popular approach assumes that the tetraquarks and pentaquarks are meson-meson and mesonbaryon bound states, respectively, formed by an attractive residual van der Waals force generated by mesonic exchanges [46, 126, 283 286]. This hypothesis is in part supported by the closeness of the observed exotic hadron masses to their respective two-particle thresholds in many cases leading to a very small binding energy, which imparts the exotic hadrons with very large hadronic radii, following from the Heisenberg uncertainty principle. A good illustration is given by the $X(3872)$, which has an $S$-wave coupling to $D^{*} \bar{D}$ (and its conjugate) and has a binding energy $\mathcal{E}_{X}=M_{X(3872)}-M_{D^{* 0}}-M_{\bar{D}^{0}}=$ $+0.01 \pm 0.18 \mathrm{MeV}$. As mentioned in 4.1.1, such a hadron molecule will have a large mean square separation of the constituents $\left\langle r>\geq 31.7_{-24.5}^{+\infty} \mathrm{fm}\right.$. This would lead to small production cross-sections in hadronic collisions [287], contrary to what has been observed in a number of experiments at the Tevatron and the LHC. In some theoretical constructs, this problem is mitigated by invoking a hard (point-like) core for the hadron molecules. In that sense, such models resemble hadroquarkonium models, discussed above. In yet others, rescattering effects are invoked to substantially increase the cross-sections [288]. A crucial test is the $p_{T}$-spectrum of the exotic hadrons in question in prompt production processes at the LHC. This spectrum has been measured for the $X(3872)$ by the CMS collaboration [10 over a $p_{T}$-range of $10 \mathrm{GeV}-50 \mathrm{GeV}$, and compared to the corresponding spectrum of the $\psi^{\prime}$. The ratio of the two $p_{T}$-spectra is found to be constant over this range, within experimental errors. This strongly suggests that the $X(3872)$ is a compact hadron. 
The hadron molecular picture is plausible in explaining some other aspects of the current data, namely the lack of experimental evidence of a quartet of exotic states, almost degenerate in mass with the $X(3872)$, containing a light quark-antiquark pair $q \bar{q}, q=u, d$, leading to the formation of $I=1$ and $I=0$ multiplets. These multiplets are anticipated in the diquark picture, discussed below. However, in the molecular picture, due to the exchange of a pion providing the main binding, and pion being an isospin $I=1$ meson, not all isospin configurations will bind. In line with this, no resonant structure is seen near the $D^{0} \bar{D}^{0}$ threshold, consistent with the inadmissibility of a strong interaction coupling of three pseudo-scalars $D^{0} \bar{D}^{0} \pi^{0}$ which violates parity conservation. More data is needed to observe or rule out the isospin partners of the $X(3872)$. On the other hand, the case for hadron molecules is less compelling for those exotics whose masses are well above the respective thresholds. For example, the $Z_{c}(3900)^{+}$is a case in point whose mass lies $20 \mathrm{MeV}$ above the $D \bar{D}^{*}$ threshold, which incidentally is also its main decay mode. This is hard to accommodate in the hadron molecular picture. Theoretical interest in hadron molecules has remained unabated, and there exists a vast and growing literature on this topic with ever increasing sophistication, a sampling of which is referenced here 289 295].

\subsection{Tetraquarks as compact diquark-antidiquark mesons}

Last on this list are QCD-based interpretations in which tetraquarks and pentaquarks are genuinely new hadron species in which a color-nonsinglet diquark is the essential building block [38 40]. In the large $N_{c}$ limit of QCD, tetraquarks, treated as diquark-antidiquark mesons, have been shown to exist 296 298 as poles in the S-matrix. They may have narrow widths in this approximation, and hence they are reasonable candidates for multiquark states. First attempts to study multiquark states using Lattice QCD have been undertaken [70,71, 299 301 in which correlations involving four-quark operators are studied numerically. Evidence of tetraquark states in the sense of S-matrix poles using these methods is still lacking. Establishing the signal of a resonance requires good control of the background. In the lattice QCD simulations of multiquark states, this is currently not the case. This may be traced back to the presence of a number of nearby hadronic thresholds and to lattice-specific issues, such as an unrealistic pion mass. More powerful analytic and computational techniques are needed to draw firm conclusions.

In the absence of reliable first principle calculations, approximate phenomenological methods are the way forward. In that spirit, an effective Hamiltonian approach has been often used [38, 39, 302, 305], in which tetraquarks are assumed to be diquark-antidiquark objects, bound by gluonic exchanges (pentaquarks are diquark-diquark-antiquark objects). The diquarks are bound by the spin-spin interaction between the two quarks of a diquark (or between two antiquarks of an anti-diquark). Motivated by the phenomenologically successful constituent quark model, the constituent diquark model allows one to work out the spectroscopy and some aspects of tetraquark decays. Heavy quark symmetry is a help in that it can be used for the heavy-light diquarks relating the charmonia-like states to the bottomoniumlike counterparts, and also in the characteristics of $b$-baryon decays leading to pentaquarks. As detailed below, diquark models anticipate a very rich spectroscopy of tetraquarks and pentaquarks, only a small part of which has been possibly observed experimentally. Hence, diquark models are in dire need of dynamical selection rules to restrict the number of observable states. The underlying multiquark dynamics is complex and the effective Hamiltonian framework, in which the parameters are assumed to subsume the dynamics, is obviously inadequate. Salient features of the phenomenology of the diquark picture are discussed below to test how far such models go in describing the observed features of the exotic hadrons measured in current experiments.

For recent in-depth reviews of all the models discussed above and the theoretical techniques employed see 13 15, 53, 306. 


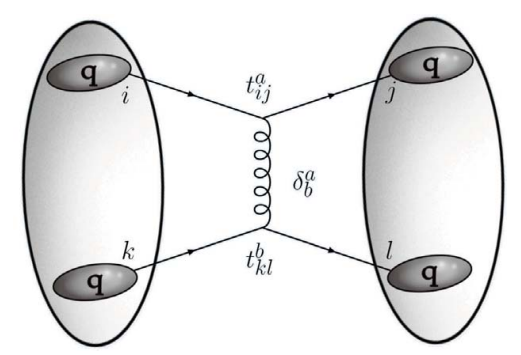

Figure 43: One-gluon exchange diagram for diquarks.

\subsection{The diquark model}

The basic assumption of this model is that diquarks are tightly bound colored objects and they are the building blocks for forming tetraquark mesons and pentaquark baryons. Diquarks, for which we use the notation $[q q]_{c}$, and interchangeably $\mathcal{Q}$, have two possible $\mathrm{SU}(3)$-color representations. Since quarks transform as a triplet 3 of color SU(3), the diquarks resulting from the direct product $3 \otimes 3=\overline{3} \oplus 6$ are thus either a color anti-triplet $\overline{3}$ or a color sextet 6 . The leading diagram based on one-gluon exchange is shown in Fig. 43 .

The product of the SU(3)-matrices in Fig. 43 can be decomposed as

$$
t_{i j}^{a} t_{k l}^{a}=-\frac{2}{3} \underbrace{\left(\delta_{i j} \delta_{k l}-\delta_{i l} \delta_{k j}\right) / 2}_{\text {antisymmetric: projects }}+\frac{1}{3} \underbrace{\left(\delta_{i j} \delta_{k l}+\delta_{i l} \delta_{k j}\right) / 2}_{\text {symmetric: projects } 6} .
$$

The coefficient of the antisymmetric $\overline{3}$ representation is $-2 / 3$, reflecting that the two diquarks bind with a strength half as strong as between a quark and an antiquark, in which case the corresponding coefficient is $-4 / 3$. The symmetric 6 on the other hand has a positive coefficient, $+1 / 3$, reflecting a repulsion. Thus, in working out the phenomenology, a diquark is assumed to be an $S U(3)_{c}$-antitriplet, with the antidiquark a color-triplet. With this, we have two color-triplet fields, quark $q_{3}$ and antidiquark $\overline{\mathcal{Q}}$ or $[\bar{q} \bar{q}]_{3}$, and two color-antitriplet fields, antiquark $\bar{q}_{\overline{3}}$ and diquark $\mathcal{Q}$ or $[q q]_{\overline{3}}$, from which the spectroscopy of the conventional and exotic hadrons is built. However, the quarks and diquarks differ in an essential detail, namely the former are point-like objects but the latter are composite and have a hadronic size. This is of crucial importance in determining the electromagnetic and strong couplings, and hence for the production cross-sections of multiquark states in leptonic and hadronic collisions.

Since quarks are spin-1/2 objects, a diquark has two possible spin-configurations, spin-0, with the two quarks in a diquark having their spin-vectors anti-parallel, and spin-1, in which case the two quark spins are aligned, as shown in Fig. 44. They were given the names "good diquarks" and "bad diquarks", respectively, by Jaffe [307], implying that in the former case, the two quarks bind, and in the latter, the binding is not as strong. There is some support of this pattern from lattice simulations for light diquarks [308], in which correlations are studied in terms of the spatial distribution of the two quarks forming the diquark in the background of the static quark. Phenomenological expectations that QCD dynamics favors the formation of good (spin-0) diquarks in color anti-triplet configuration is verified.

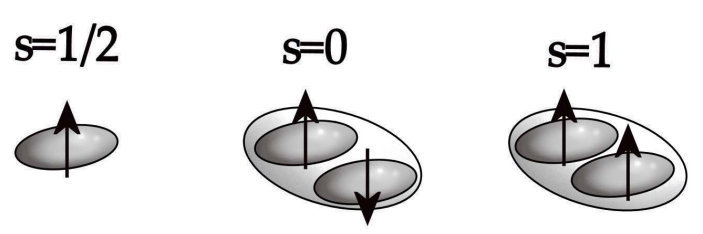

Figure 44: Quark and diquark spins. 
It is exceedingly important to study on the lattice such correlations in two-point functions, involving tetraquarks, which have been attempted but with inconclusive results so far. However, as the spin-degree of freedom decouples in the heavy quark systems, as can be shown explicitly in the heavy quark effective theory context for heavy mesons and baryons [309], we expect that this decoupling will also hold for heavy-light diquarks $\left[Q_{i} q_{j}\right]_{3}$ with $Q_{i}=c, b ; q_{j}=u, d, s$. So, for the heavy-light diquarks, both the spin-1 and spin-0 configurations are phenomenologically present. Also, the diquarks in heavy baryons (such as $\Lambda_{b}$ and $\Omega_{b}$ ), consisting of a heavy quark and a light diquark, both $J^{p}=0^{+}$and $J^{p}=1^{+}$quantum numbers of the diquark are needed to accommodate the observed baryon spectrum. In this review, we restrict ourselves to the heavy-light diquarks, though heavy-heavy diquarks $[Q Q]_{\overline{3}}(Q=c, b)$, and the resulting tetraquark states $[Q Q]_{3}[\bar{Q} \bar{Q}]_{3}$ are also anticipated and discussed in the literature [310].

Following the discussion above, we construct the interpolating diquark operators for the two spinstates of such diquarks (here $Q=c, b)[39$ :

$$
\begin{aligned}
& \text { Scalar } \quad 0^{+}: \quad \mathcal{Q}_{i \alpha}=\epsilon_{\alpha \beta \gamma}\left(\bar{Q}_{c}^{\beta} \gamma_{5} q_{i}^{\gamma}-\bar{q}_{i_{c}}^{\beta} \gamma_{5} Q^{\gamma}\right), \quad \alpha, \beta, \gamma: S U(3)_{C} \text { indices } \\
& \text { Axial-Vector } 1^{+}: \quad \overrightarrow{\mathcal{Q}}_{i \alpha}=\epsilon_{\alpha \beta \gamma}\left(\bar{Q}_{c}^{\beta} \vec{\gamma} q_{i}^{\gamma}+\bar{q}_{i_{c}}^{\beta} \vec{\gamma} Q^{\gamma}\right) \text {. }
\end{aligned}
$$

In the non-relativistic (NR) limit, these states are parametrized by Pauli matrices: $\Gamma^{0}=\frac{\sigma_{2}}{\sqrt{2}}$ for the Scalar $\left(0^{+}\right)$, and $\vec{\Gamma}=\frac{\sigma_{2} \vec{\sigma}}{\sqrt{2}}$ for the Axial-Vector $\left(1^{+}\right)$. A tetraquark state with total angular momentum $J$ may be described by the state vector $\left|s_{\mathcal{Q}}, s_{\overline{\mathcal{Q}}} ; J\right\rangle$ showing the diquark spin $s_{\mathcal{Q}}$ and the antidiquark spin $s_{\overline{\mathcal{Q}}}$. There is no consensus on their names. We use the symbols $X_{J}$ for $J^{++}, Z$ for $J^{P C}=1^{+-}$, and $Y$ for $J^{P C}=1^{--}$states. Thus, the tetraquarks with the following diquark-spin and angular momentum $J$ have the Pauli forms:

$$
\begin{aligned}
\left|0_{\mathcal{Q}}, 0_{\overline{\mathcal{Q}}} ; 0_{J}\right\rangle & =\Gamma^{0} \otimes \Gamma^{0}, \\
\left|1_{\mathcal{Q}}, 1_{\overline{\mathcal{Q}}} ; 0_{J}\right\rangle & =\frac{1}{\sqrt{3}} \Gamma^{i} \otimes \Gamma_{i}, \\
\left|0_{\mathcal{Q}}, 1_{\overline{\mathcal{Q}}} ; 1_{J}\right\rangle & =\Gamma^{0} \otimes \Gamma^{i}, \\
\left|1_{\mathcal{Q}}, 0_{\overline{\mathcal{Q}}} ; 1_{J}\right\rangle & =\Gamma^{i} \otimes \Gamma^{0} \\
\left|1_{\mathcal{Q}}, 1_{\overline{\mathcal{Q}}} ; 1_{J}\right\rangle & =\frac{1}{\sqrt{2}} \varepsilon^{i j k} \Gamma_{j} \otimes \Gamma_{k}
\end{aligned}
$$

Whenever necessary, a subscript $c$ or $b$ is used to distinguish the $c \bar{c}$ and $b \bar{b}$ states.

\subsection{Non-relativistic Hamiltonian for tetraquarks with hidden charm}

For the heavy quarkonium-like exotic hadrons the non-relativistic limit is a good approximation. This effective NR Hamiltonian has been proposed to calculate the tetraquark mass spectrum 39,302

$$
H_{\mathrm{eff}}=2 m_{\mathcal{Q}}+H_{S S}^{(q q)}+H_{S S}^{(q \bar{q})}+H_{S L}+H_{L L}
$$

where $m_{\mathcal{Q}}$ is the constituent diquark mass, the second term above is the spin-spin interaction involving the quarks (or antiquarks) in a diquark (or anti-diquark), the third term depicts spin-spin interactions involving a quark and an antiquark in two different shells (i.e., in the two different diquark configurations), with the fourth and fifth terms being the spin-orbit and the orbit-orbit interactions, involving the quantum numbers of the tetraquark, respectively. For the $S$-states, these last two terms are absent. For $Q=c$, the individual terms in $H_{\text {eff }}$ are displayed below and illustrated in Fig. 45 .

$$
\begin{gathered}
H_{S S}^{(q q)}=2\left(\mathcal{K}_{c q}\right) \overline{3}\left[\left(\mathbf{S}_{c} \cdot \mathbf{S}_{q}\right)+\left(\mathbf{S}_{\bar{c}} \cdot \mathbf{S}_{\bar{q}}\right)\right] \\
H_{S S}^{(q \bar{q})}=2\left(\mathcal{K}_{c \bar{q}}\right)\left(\mathbf{S}_{c} \cdot \mathbf{S}_{\bar{q}}+\mathbf{S}_{\bar{c}} \cdot \mathbf{S}_{q}\right)+2 \mathcal{K}_{c \bar{c}}\left(\mathbf{S}_{c} \cdot \mathbf{S}_{\bar{c}}\right)+2 \mathcal{K}_{q \bar{q}}\left(\mathbf{S}_{q} \cdot \mathbf{S}_{\bar{q}}\right) \\
H_{S L}=2 A_{\mathcal{Q}}\left(\mathbf{S}_{\mathcal{Q}} \cdot \mathbf{L}+\mathbf{S}_{\overline{\mathcal{Q}}} \cdot \mathbf{L}\right) \\
H_{L L}=B_{\mathcal{Q}} \frac{L_{\mathcal{Q}}\left(L_{\mathcal{Q}}+1\right)}{2}
\end{gathered}
$$




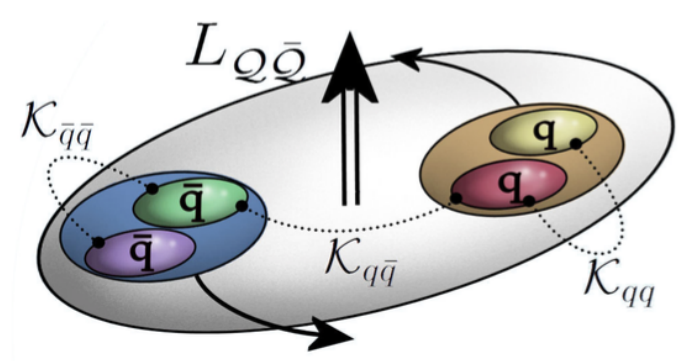

Figure 45: Schematic diagram of a tetraquark in the diquark-antidiquark picture.

Table 17: $S$-wave tetraquark states involving a $Q \bar{Q}$ pair in the two bases and their masses in the diquark model.

\begin{tabular}{ccccc}
\hline \hline Label & $J^{P C}$ & $\left|s_{q Q}, s_{\bar{q} \bar{Q}} ; S, L\right\rangle_{J}$ & $\left|s_{q \bar{q}}, s_{Q \bar{Q}} ; S^{\prime}, L^{\prime}\right\rangle_{J}$ & Mass \\
\hline$X_{0}$ & $0^{++}$ & $|0,0 ; 0,0\rangle_{0}$ & $\left(|0,0 ; 0,0\rangle_{0}+\sqrt{3}|1,1 ; 0,0\rangle_{0}\right) / 2$ & $M_{00}-3 \kappa_{q Q}$ \\
$X_{0}^{\prime}$ & $0^{++}$ & $|1,1 ; 0,0\rangle_{0}$ & $\left(\sqrt{3}|0,0 ; 0,0\rangle_{0}-|1,1 ; 0,0\rangle_{0}\right) / 2$ & $M_{00}+\kappa_{q Q}$ \\
$X_{1}$ & $1^{++}$ & $\left(|1,0 ; 1,0\rangle_{1}+|0,1 ; 1,0\rangle_{1}\right) / \sqrt{2}$ & $\left|1,1 ; 1, L^{\prime}\right\rangle_{1}$ & $M_{00}-\kappa_{q Q}$ \\
$Z$ & $1^{+-}$ & $\left(|1,0 ; 1,0\rangle_{1}-|0,1 ; 1,0\rangle_{1}\right) / \sqrt{2}$ & $\left(\left|1,0 ; 1, L^{\prime}\right\rangle_{1}-\left|0,1 ; 1, L^{\prime}\right\rangle_{1}\right) / \sqrt{2}$ & $M_{00}-\kappa_{q Q}$ \\
$Z^{\prime}$ & $1^{+-}$ & $|1,1 ; 1,0\rangle_{1}$ & $\left(\left|1,0 ; 1, L^{\prime}\right\rangle_{1}+\left|0,1 ; 1, L^{\prime}\right\rangle_{1}\right) / \sqrt{2}$ & $M_{00}+\kappa_{q Q}$ \\
$X_{2}$ & $2^{++}$ & $|1,1 ; 2,0\rangle_{2}$ & $\left|1,1 ; 2, L^{\prime}\right\rangle_{2}$ & $M_{00}+\kappa_{q Q}$ \\
\hline \hline
\end{tabular}

Here $\left(\mathcal{K}_{c q}\right)_{\overline{3}}$ parametrizes the spin-spin interaction between the charm $c$ and the light quark $q$ within a diquark in a color anti-triplet configuration, and the various $\left(\mathcal{K}_{i \bar{j}}\right)$ parametrize the spin-spin strengths between the quarks $i$ and the antiquark $\bar{j}$, in the color-singlet configuration involving two different diquarks. The parameters $A_{\mathcal{Q}}$ and $B_{\mathcal{Q}}$ characterize the strength of the spin-orbit and the orbital angular force, respectively.

The usual angular momentum algebra then yields the following form:

$$
\begin{aligned}
H_{\mathrm{eff}} & =2 m_{\mathcal{Q}}+\frac{B_{\mathcal{Q}}}{2}\left\langle L^{2}\right\rangle-2 a\langle L \cdot S\rangle+2 \kappa_{q c}\left[\left\langle s_{q} \cdot s_{c}\right\rangle+\left\langle s_{\bar{q}} \cdot s_{\bar{c}}\right\rangle\right] \\
& =2 m_{\mathcal{Q}}-a J(J+1)+\left(\frac{B_{\mathcal{Q}}}{2}+a\right) L(L+1)+a S(S+1)-3 \kappa_{q c} \\
& +\kappa_{q c}\left[s_{q c}\left(s_{q c}+1\right)+s_{\bar{q} \bar{c}}\left(s_{\bar{q} \bar{c}}+1\right)\right] .
\end{aligned}
$$

The effective Hamiltonian given above can be used for the tetraquark states involving a $b \bar{b}$ pair, with appropriate rescaling of the parameters.

\subsection{Low-lying $S$ and $P$-wave tetraquark states in the $c \bar{c}$ and $b \bar{b}$ sectors}

The states in the diquark-antidiquark basis $\left|s_{q Q}, s_{\bar{q} \bar{Q}} ; S, L\right\rangle_{J}$ and in the $Q \bar{Q}$ and $q \bar{q}$ basis $\left|s_{q \bar{q}}, s_{Q \bar{Q}} ; S^{\prime}, L^{\prime}\right\rangle_{J}$ are related by Fierz transformation. The positive parity $S$-wave tetraquarks are given in terms of the six states listed in Table 17 (charge conjugation is defined for neutral states). These states are characterized by the quantum number $L=0$, hence their masses depend on just two parameters the diquark mass, $M_{00}$, and $\kappa_{q Q}$, leading to several testable predictions. The $P$-wave states are listed in Table 18 . The first four of them have $L=1$, and the fifth has $L=3$, and hence is expected to be significantly heavier.

The parameters appearing on the right-side columns of Tables 17 and 18 can be determined using the masses of some of the observed $X, Y, Z$ states, and their numerical values are given in Table 19 . Some parameters in the $c \bar{c}$ and $b \bar{b}$ sectors can also be related using the heavy quark mass scaling [311]. 
Table 18: $P$-wave $\left(J^{P C}=1^{--}\right)$tetraquark states involving a $Q \bar{Q}$ pair in the two bases and their masses in the diquark model.

\begin{tabular}{cccc}
\hline \hline Label & $\left|s_{q Q}, s_{\bar{q} \bar{Q}} ; S, L\right\rangle_{J}$ & $\left|s_{q \bar{q}}, s_{Q \bar{Q}} ; S^{\prime}, L^{\prime}\right\rangle_{J}$ & Mass \\
\hline$Y_{1}$ & $|0,0 ; 0,1\rangle_{1}$ & $\left(|0,0 ; 0,1\rangle_{1}+\sqrt{3}|1,1 ; 0,1\rangle_{1}\right) / 2$ & $M_{00}-3 \kappa_{q Q}+B_{Q}$ \\
$Y_{2}$ & $\left(|1,0 ; 1,1\rangle_{1}+|0,1 ; 1,1\rangle_{1}\right) / \sqrt{2}$ & $\left|1,1 ; 1, L^{\prime}\right\rangle_{1}$ & $M_{00}-\kappa_{q Q}+2 a+B_{Q}$ \\
$Y_{3}$ & $|1,1 ; 0,1\rangle_{1}$ & $\left(\sqrt{3}|0,0 ; 0,1\rangle_{1}-|1,1 ; 0,1\rangle_{1}\right) / 2$ & $M_{00}+\kappa_{q Q}+B_{Q}$ \\
$Y_{4}$ & $|1,1 ; 2,1\rangle_{1}$ & $\left|1,1 ; 2, L^{\prime}\right\rangle_{1}$ & $M_{00}+\kappa_{q Q}+6 a+B_{Q}$ \\
$Y_{5}$ & $|1,1 ; 2,3\rangle_{1}$ & $\left|1,1 ; 2, L^{\prime}\right\rangle_{1}$ & $M_{00}+\kappa_{q Q}+16 a+6 B_{Q}$ \\
\hline \hline
\end{tabular}

Table 19: Numerical values of the parameters in $H_{\text {eff }}$, obtained using some of the $S$ and $P$-wave tetraquarks as input.

\begin{tabular}{c|cc}
\hline \hline & charmonium-like & bottomonium-like \\
\hline$M_{00}[\mathrm{MeV}]$ & 3957 & 10630 \\
$\kappa_{q Q}[\mathrm{MeV}]$ & 67 & 23 \\
$B_{Q}[\mathrm{MeV}]$ & 268 & 329 \\
$a[\mathrm{MeV}]$ & 52.5 & 26 \\
\hline \hline
\end{tabular}

Table 20: $J^{P C}$ quantum numbers of the $X, Y, Z$ exotic hadrons and their masses from experiments and in the diquark-model.

\begin{tabular}{c|c|cc|cc}
\hline \hline & & \multicolumn{2}{|c|}{ charmonium-like } & \multicolumn{2}{c}{ bottomonium-like } \\
Label & $J^{P C}$ & State & Mass $[\mathrm{MeV}]$ & State & Mass $[\mathrm{MeV}]$ \\
\hline$X_{0}$ & $0^{++}$ & - & 3756 & - & 10562 \\
$X_{0}^{\prime}$ & $0^{++}$ & - & 4024 & - & 10652 \\
$X_{1}$ & $1^{++}$ & $X(3872)$ & 3890 & - & 10607 \\
$Z$ & $1^{+-}$ & $Z_{c}^{+}(3900)$ & 3890 & $Z_{b}^{+, 0}(10610)$ & 10607 \\
$Z^{\prime}$ & $1^{+-}$ & $Z_{c}^{+}(4020)$ & 4024 & $Z_{b}^{+}(10650)$ & 10652 \\
$X_{2}$ & $2^{++}$ & - & 4024 & - & 10652 \\
\hline$Y_{1}$ & $1^{--}$ & $Y(4008)$ & 4024 & $Y_{b}(10891)$ & 10891 \\
$Y_{2}$ & $1^{--}$ & $Y(4260)$ & 4263 & $Y_{b}(10987)$ & 10987 \\
$Y_{3}$ & $1^{--}$ & $Y(4290)($ or $Y(4220))$ & 4292 & - & 10981 \\
$Y_{4}$ & $1^{--}$ & $Y(4630)$ & 4607 & - & 11135 \\
$Y_{5}$ & $1^{--}$ & - & 6472 & - & 13036 \\
\hline \hline
\end{tabular}


There are several predictions in the charmonium-like sector, which, with the values of the parameters given in the tables above, are in the right ball-park. In a modified scheme [302], developed subsequently, the parameters $\left(\kappa_{i \bar{j}}\right)$ are set to zero, eliminating the $H_{S S}^{(q \bar{q})}$ term in the effective Hamiltonian. With this, better agreement is reached with experiments. Despite this success, the continued experimental absence of the two lowest-lying $0^{++}$states, called $X_{0}$ and $X_{0}^{\prime}$, is puzzling. Perhaps, they are below the threshold for strong decays and decay weakly, and thus have not been looked for. Alternative calculations of the tetraquark spectrum based on diquark-antidiquark model have been carried out in other phenomenological schemes [312], and in the QCD sum rule framework [313, 314]. All of them share the common feature with the effective Hamiltonian approach discussed here, namely they all anticipate a very rich tetraquark spectroscopy. So, if the diquark picture has come to stay, some dynamical selection rules are required to better understand the observed spectrum.

The exotic bottomonium-like states are currently rather sparse. The reason for this is that quite a few exotic candidate charmonium-like states were observed in the decays of $B$ hadrons. The observed pentaquark states $P_{c}(4380)^{+}$and $P_{c}(4450)^{+}$are decay products of the $\Lambda_{b}$-baryon. However, as the top quark decays weakly before it gets a chance to hadronize, exotic hadrons with a hidden $b \bar{b}$ pair are not anticipated from the top quark decays. Hence, they can only be produced in hadro- and electroweak high energy processes, which makes their detection lot harder. Tetraquark states with a single $b$ quark can, in principle, be searched for in the hadronic debris of a $b$-quark initiated jet, or in the decays of the $B_{c}$ mesons [315]. As the $c \bar{c}$ and $b \bar{b}$ cross-section at the LHC are very large, we anticipate that the exotic spectroscopy involving the open and hidden heavy quarks is an area where significant new results will be reported by all the LHC experiments. Measurements of the production and decay characteristics of exotica, such as the transverse-momentum distributions and polarization information, will go a long way in understanding the underlying dynamics.

We now discuss the three candidate exotic states observed so far in the bottomonium sector. The hidden $b \bar{b}$ state $Y_{b}(10890)$ with $J^{\mathrm{P}}=1^{--}$was discovered by Belle in 2007 [230] in the process $e^{+} e^{-} \rightarrow Y_{b}(10890) \rightarrow(\Upsilon(1 S), \Upsilon(2 S), \Upsilon(3 S)) \pi^{+} \pi^{-}$just above the $\Upsilon(5 S)$. The branching ratios measured for these decays are about two orders of magnitude larger than anticipated from similar dipionic transitions in the lower $\Upsilon(n S)$ states and in the $\psi^{\prime}$ (for a review and references to earlier work, see Brambilla et al. [20]). Also the dipion invariant mass distributions in the decays of $Y_{b}$ are marked by the presence of the resonances $f_{0}(980)$ and $f_{2}(1270)$. This state was interpreted as a $J^{\mathrm{PC}}=1^{--} P$-wave tetraquark 303, 304]. Subsequent to this, a Van Royen-Weiskopf formalism was used [305] in which direct electromagnetic couplings with the diquark-antidiquark pair of the $Y_{b}$ was assumed. Due to the $P$-wave nature of the $Y_{b}(10890)$, and the hadronic size of the diquarks, the effective electromagnetic coupling is reduced. Hence, the production cross-section for $e^{+} e^{-} \rightarrow Y_{b}(10890) \rightarrow b \bar{b}$ is anticipated to be small, leading only to a small bump in the $R_{b}$-scan. However, due to the presence of a $b \bar{b}$ and a light $q \bar{q}(q=u, d)$ pair in $Y_{b}(10890)$ in the valence approximation, the decays $Y_{b}(10890) \rightarrow \Upsilon(n S) \pi^{+} \pi^{-}$are Zweig-allowed. Since, there is practically no background to these final states from the continuum $e^{+} e^{-}$ annihilation, as opposed to $e^{+} e^{-} \rightarrow b \bar{b}$, a bump in the $e^{+} e^{-} \rightarrow \Upsilon(n S) \pi^{+} \pi^{-}$due to $Y_{b}(10890)$ should be visible. This is, at least qualitatively, in agreement with the Belle data [316].

The model in which direct electromagnetic coupling with a diquark-antidiquark pair is envisaged has experimentally testable consequences. Among other implications, one expects large isospin-breaking effects, arising from the different electric charges of the $[b u]$ and $[b d]$ diquarks. In the tetraquark picture, the $Y_{b}(10890)$ is the $b \bar{b}$ analogue of the $c \bar{c}$ state $Y(4260)$, also a $P$-wave, which is likewise found to have a very small production cross-section in $e^{+} e^{-} \rightarrow Y(4260) \rightarrow c \bar{c}$, but which decays readily into $J / \psi \pi^{+} \pi^{-}$, reflecting the presence of a $c \bar{c}$ and a light $q \bar{q}$ pair in the Fock space of the $Y(4260)$. Hence, the two $Y$-states have very similar production and decay characteristics, and both are $J^{P C}=1^{--}$tetraquark candidates. There are other production mechanisms for tetraquarks in which the electromagnetic current couples with the $c \bar{c}$ or $b \bar{b}$ quark pair, which before decaying picks up a light 
$q \bar{q}$ from the vacuum, resulting in a diquark-antidiquark pair. If the center-of-mass energy is close to a $J^{P C}=1^{--}$tetraquark mass, one expects a resonant production. However, the cross-section, due to the angular momentum barrier reflecting the $P$-wave nature of the tetraquark, and a small probability for the fragmentation $c \bar{c} \rightarrow[c q][\bar{c} \bar{q}]$ (or $b \bar{b} \rightarrow[b q][\bar{b} \bar{q}]$ ), is expected to be small. In this case, as opposed to the direct electromagnetic coupling to the diquark-antidiquark pair, the production mechanism will respect isospin symmetry.

The current status of $Y_{b}(10890)$ is, however, unclear. Subsequent to its discovery, Belle undertook high-statistics scans to measure the ratio $R_{b \bar{b}}=\sigma\left(e^{+} e^{-} \rightarrow b \bar{b}\right) / \sigma\left(e^{+} e^{-} \rightarrow \mu^{+} \mu^{-}\right)$, and also more precisely the ratios $R_{\Upsilon(n S) \pi^{+} \pi^{-}}=\sigma\left(e^{+} e^{-} \rightarrow \Upsilon(n S) \pi^{+} \pi^{-}\right) / \sigma\left(e^{+} e^{-} \rightarrow \mu^{+} \mu^{-}\right)$. They are shown in Fig. 46 and Fig. 47, respectively. The two masses, $M(5 S)_{b \bar{b}}$ measured through $R_{b \bar{b}}$, and $M\left(Y_{b}\right)$ measured through $R_{\Upsilon(n S) \pi^{+} \pi^{-}}$, now differ by slightly more than $2 \sigma, M(5 S)_{b \bar{b}}-M\left(Y_{b}\right)=-9 \pm 4 \mathrm{MeV}$. From the mass difference alone, these two could very well be just one and the same state, namely the canonical $\Upsilon(5 S)$ - an interpretation now adopted by the Belle collaboration [316]. On the other hand, it is the bookkeeping of the branching ratios measured at or near the " $(5 \bar{S})$ ", which is enigmatic. The branching ratios of the " $\Upsilon(5 S)$ " measured by Belle are saturated by the exotic states $\left(\Upsilon(n S) \pi^{+} \pi^{-}, h_{b}(m P) \pi^{+} \pi^{-}, Z_{b}(10610)^{ \pm} \pi^{\mp}, Z_{b}(10650)^{ \pm} \pi^{\mp}\right.$ and their isospin partners). Another class of states consists of $\left[B^{*} B^{(*)}\right]^{ \pm} \pi^{\mp}$, which is found to originate exclusively from the $Z_{b}(10610)^{ \pm} \pi^{\mp}$ and $Z_{b}(10650)^{ \pm} \pi^{\mp}$. Based on these measurements and assuming isospin symmetry, Belle has reported a cumulative value $\mathcal{P}=1.09 \pm 0.15$ [316], where a value of $\mathcal{P}=1$ corresponds to the saturation of the " $5 \mathrm{~S}$ " amplitude by the contributing exotic channels (listed above). This leaves little room at "5S" for other known final states, such as $B_{(s)}^{(*)} \bar{B}_{(s)}^{(*)}$, despite the fact these reactions have large cross sections measured in independent experiments, first reported by CLEO [12] and more recently by Belle itself.

The reason for this mismatch is not clear; Belle attributes it to the inadequate modeling of $R_{b}$ due to several thresholds in this energy region. While this may eventually be the source of the current " $\Upsilon(5 S)$ " branching ratio puzzle, an interpretation of the Belle data based on two almost degenerate (in mass) resonances $\Upsilon(5 S)$ and $Y_{b}(10890)$ is also a logical possibility, with $\Upsilon(5 S)$ having the decays expected for the bottomonium $S$-state above the $B^{(*)} \bar{B}^{(*)}$ threshold, and the decays of $Y_{b}(10890)$, a tetraquark, being the source of the exotic states seen. On the other hand, no peaking structure at $10.9 \mathrm{GeV}$ in

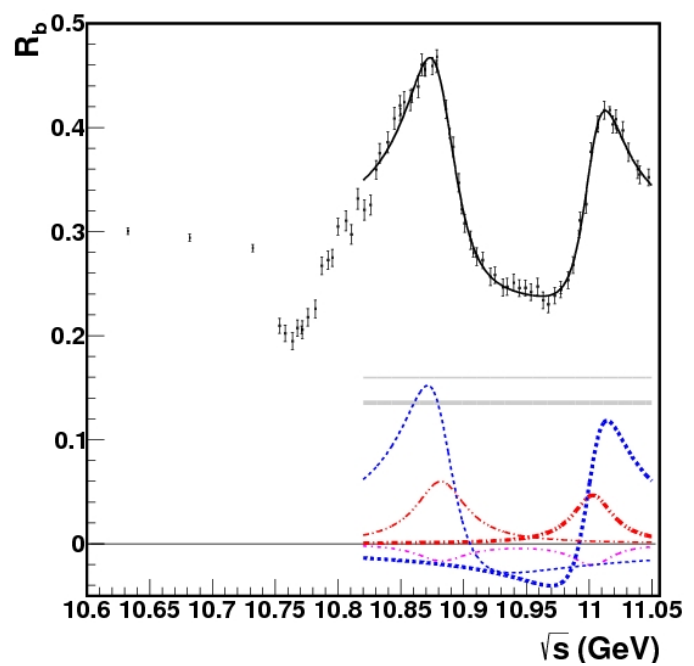

Figure 46: The ratio $R_{b}=\sigma\left(e^{+} e^{-} \rightarrow b \bar{b}\right) / \sigma\left(e^{+} e^{-} \rightarrow \mu^{+} \mu^{-}\right)$in the $Y(10860)$ and $Y(11020)$ region. The components of the fit are depicted in the lower part of the figure: total (solid curve), constant $\left|A_{i c}\right|^{2}$ (thin), $\left|A_{c}\right|^{2}$ (thick): for $\Upsilon(5 S)$ (thin) and $\Upsilon(6 S)$ (thick): $|f|^{2}$ (dot-dot-dash), cross terms with $A_{c}$ (dashed), and two-resonance cross term (dot-dash). Here, $A_{c}$ and $A_{i c}$ are coherent and incoherent continuum terms, respectively (from Belle [316]). 


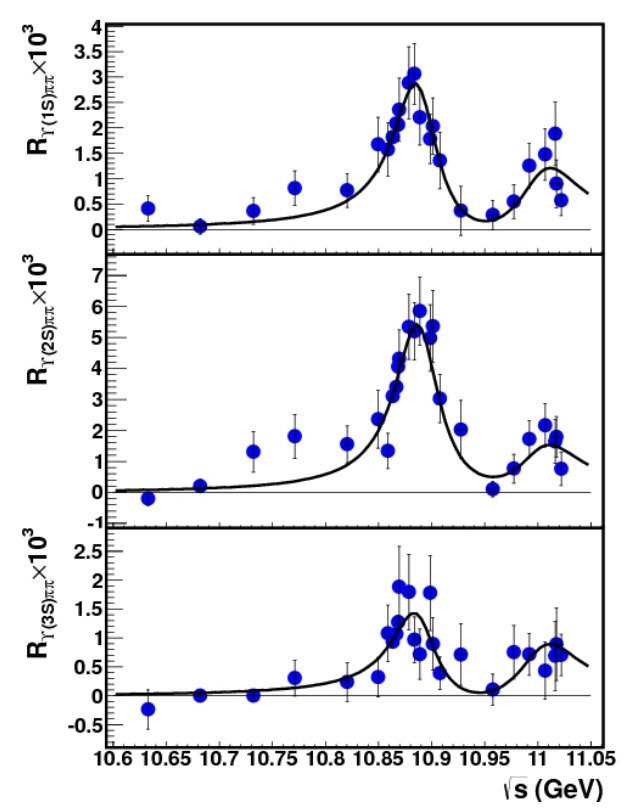

Figure 47: The ratio $R_{\Upsilon(n S) \pi^{+} \pi^{-}}=\sigma\left(e^{+} e^{-} \rightarrow \Upsilon(n S) \pi^{+} \pi^{-}\right) / \sigma\left(e^{+} e^{-} \rightarrow \mu^{+} \mu^{-}\right)$in the $Y(10860)$ and $Y(11020)$ region (from Belle [316]).

the $R_{b}$ distribution is seen in the Belle analysis, and an upper limit on $\Gamma_{e e}$ of $9 \mathrm{eV}$ is set with a $90 \%$ confidence level [316]. As data taking starts in a couple of years in the form of a new and expanded collaboration, Belle-II, cleaning up the current analysis in the $\Upsilon(5 S)$ and $\Upsilon(6 S)$ regions should be one of their top priorities.

The hypothesis that $\Upsilon(5 S)$ and $Y_{b}(10890)$, while having the same $J^{\mathrm{PC}}=1^{--}$quantum numbers and almost the same mass, are different states, is also hinted by the drastically different decay characteristics of the dipionic transitions involving the lower quarkonia $S$-states, such as $\Upsilon(4 S) \rightarrow \Upsilon(1 S) \pi^{+} \pi^{-}$, on one hand, and similar decays of the $Y_{b}$, on the other. These anomalies are seen both in the decay rates and in the dipion invariant mas spectra in the $\Upsilon(n S) \pi^{+} \pi^{-}$modes. The large branching ratios of $Y_{b} \rightarrow \Upsilon(n S) \pi^{+} \pi^{-}$, as well as of $Y(4260) \rightarrow J / \psi \pi^{+} \pi^{-}$, are due to the Zweig-allowed nature of these transitions, as the initial and final states have the same valence quarks. The final state $\Upsilon(n S) \pi^{+} \pi^{-}$ in $Y_{b}$ decays requires the excitation of a $q \bar{q}$ pair from the vacuum. Since, the light scalars $\sigma_{0}, f_{0}(980)$ are themselves tetraquark candidates [263, 264, they are expected to show up in the $\pi^{+} \pi^{-}$invariant mass distributions, as opposed to the corresponding spectrum in the transition $\Upsilon(4 S) \rightarrow \Upsilon(1 S) \pi^{+} \pi^{-}$ (see Fig. 48). Subsequent discoveries [27] of the charged states $Z_{b}^{+}(10610)$ and $Z_{b}^{+}(10650)$, found in the decays $\Upsilon(5 S) / Y_{b} \rightarrow Z_{b}^{+}(10610) \pi^{-}, Z_{b}^{+}(10650) \pi^{-}$, leading to the final states $\Upsilon(1 S) \pi^{+} \pi^{-}, \Upsilon(2 S) \pi^{+} \pi^{-}$, $\Upsilon(3 S) \pi^{+} \pi^{-}, h_{b}(1 P) \pi^{+} \pi^{-}$and $h_{b}(2 P) \pi^{+} \pi^{-}$, also admit a tetraquark interpretation, as discussed below.

\subsection{Heavy-Quark-Spin Flip in $\Upsilon(10890) \rightarrow h_{b}(1 P, 2 P) \pi \pi$}

The cross-section $\sigma\left(e^{+} e^{-} \rightarrow\left(h_{b}(1 P), h_{b}(2 P) \pi^{+} \pi^{-}\right)\right.$measured by Belle [30] is shown in Fig. 49, providing clear evidence of production in the $\Upsilon(10860)$ and $\Upsilon(11020)$ region. We summarize the relative rates and strong phases measured by Belle [27] in the process $\Upsilon(10890) \rightarrow \Upsilon(n S) \pi^{+} \pi^{-}, h_{b}(m P) \pi^{+} \pi^{-}$, with $n=1,2,3$ and $m=1,2$ in Table 21. We use the notation $Z_{b}$ and $Z_{b}^{\prime}$ for the two charged $Z_{b}$ states. Here no assumption is made about the nature of $\Upsilon(10890)$; it can be either $\Upsilon(5 S)$ or $Y_{b}$. Of these, the decay $\Upsilon(10890) \rightarrow \Upsilon(1 S) \pi^{+} \pi^{-}$involves both a resonant (i.e., via $Z / Z^{\prime}$ ) and a direct component, but the other four are dominated by the resonant contribution. One notices that the relative normalizations are very similar and the phases of the $(\Upsilon(2 S), \Upsilon(3 S)) \pi^{+} \pi^{-}$differ by about $180^{\circ}$ compared to the ones 


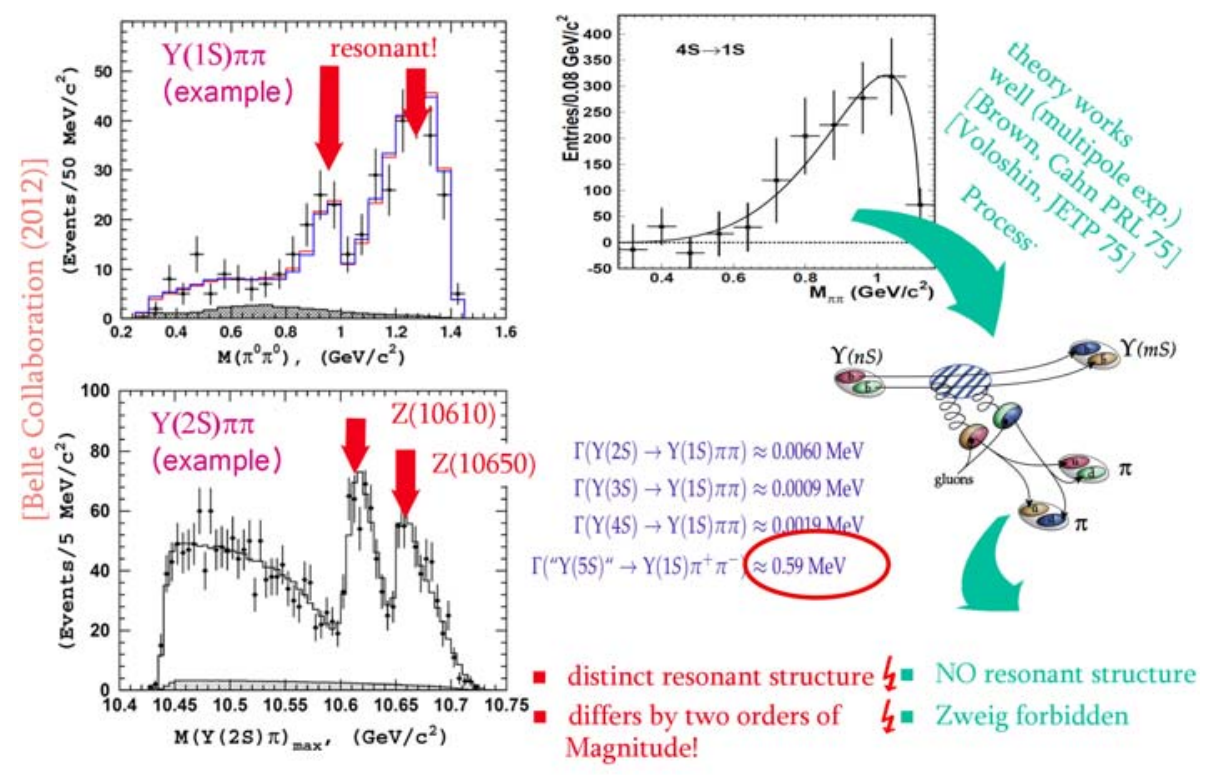

Figure 48: Dipion invariant mass distribution in $\Upsilon(10890) \rightarrow \Upsilon(1 S) \pi^{0} \pi^{0}$ (upper left frame); the resonances indicated in the dipion spectrum correspond to the $f_{0}(980)$ and $f_{2}(1270)$; the resonances $Z(10610)$ and $Z(10650)$ are indicated in the $\Upsilon(2 S) \pi^{+}$invariant mass distribution from $\Upsilon(10890) \rightarrow$ $\Upsilon(2 S) \pi^{+} \pi^{-}$(lower left frame). The data are from the Belle collaboration [27]. The upper right hand frame shows the dipion invariant mass distribution in $\Upsilon(4 S) \rightarrow \Upsilon(1 S) \pi^{+} \pi^{-}$, and the theoretical curve (with the references) is based on the Zweig-forbidden process shown below. The measured decay widths from $\Upsilon(n S) \rightarrow \Upsilon(1 S) \pi^{+} \pi^{-} n S=2 S, 3 S, 4 S$ and $\Upsilon(10890) \rightarrow \Upsilon(1 S) \pi^{+} \pi^{-}$are also shown.

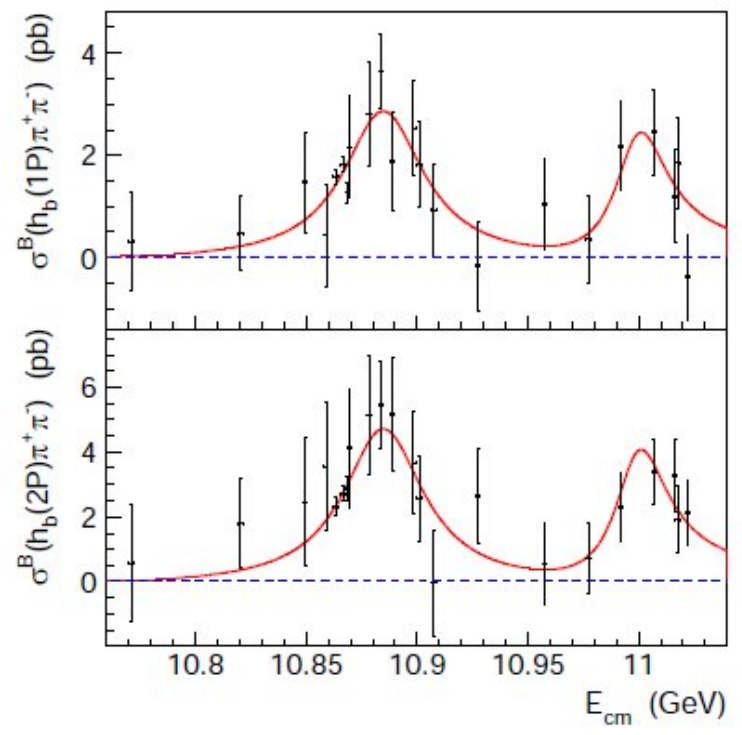

Figure 49: $\sigma\left(e^{+} e^{-} \rightarrow h_{b}(1 P) \pi^{+} \pi^{-}\right)$and $\sigma\left(e^{+} e^{-} \rightarrow h_{b}(2 P) \pi^{+} \pi^{)}\right.$-in the $Y(10860)$ and $Y(11020)$ region(from Belle $[30]$ ). 
Table 21: Relative normalizations and phases for $s_{b \bar{b}}: 1 \rightarrow 1$ and $1 \rightarrow 0$ transitions in $\Upsilon(10890)$ decays [27].

\begin{tabular}{lccccc}
\hline Final State & $\Upsilon(1 S) \pi^{+} \pi^{-}$ & $\Upsilon(2 S) \pi^{+} \pi^{-}$ & $\Upsilon(3 S) \pi^{+} \pi^{-}$ & $h_{b}(1 P) \pi^{+} \pi^{-}$ & $h_{b}(2 P) \pi^{+} \pi^{-}$ \\
\hline Rel. Norm. & $0.57 \pm 0.21_{-0.04}^{+0.19}$ & $0.86 \pm 0.11_{-0.10}^{+0.04}$ & $0.96 \pm 0.14_{-0.05}^{+0.08}$ & $1.39 \pm 0.37_{-0.15}^{+0.05}$ & $1.6_{-0.4-0.6}^{+0.6+0.4}$ \\
Rel. Phase & $58 \pm 43_{-9}^{+4}$ & $-13 \pm 13_{-8}^{+17}$ & $-9 \pm 19_{-26}^{+11}$ & $187_{-57-12}^{+44+3}$ & $181_{-105-109}^{+65+74}$ \\
\hline
\end{tabular}

in $\left(h_{b}\left(1 P, h_{b}(2 P)\right) \pi^{+} \pi^{-}\right.$. At the first sight this seems to violate the heavy-quark-spin conservation, as in the initial state $s_{b \bar{b}}=1$, which remains unchanged for the $\Upsilon(n S)$ in the final state, i.e., it involves an $s_{b \bar{b}}=1 \rightarrow s_{b \bar{b}}=1$ transition, but as $s_{b \bar{b}}=0$ for the $h_{b}(m P)$, this involves an $s_{b \bar{b}}=1 \rightarrow s_{b \bar{b}}=0$ transition, which should have been suppressed, but is not supported by data. It has been shown that this contradiction is only apparent [311].

In the tetraquark picture, one has a triplet of $J^{P G}=1^{++}$states, the $Z_{b}$ and $Z_{b}^{\prime}$, and another, yet to be discovered $X_{b}$, with $C=+1$. They have the following form in the diquark-antidiquark spin representation:

$$
\begin{aligned}
\left|Z_{b}\right\rangle & =\frac{\left|1_{b q}, 0_{\bar{b} \bar{q}}\right\rangle-\left|0_{b q}, 1_{\bar{b} \bar{q}}\right\rangle}{\sqrt{2}}, \\
\left|Z_{b}^{\prime}\right\rangle & =\left|1_{b q}, 1_{\bar{b} \bar{q}}\right\rangle_{J=1}, \\
\left|X_{b}\right\rangle & =\frac{\left|1_{b q}, 0_{\bar{b} \bar{q}}\right\rangle+\left|0_{b q}, 1_{\bar{b} \bar{q}}\right\rangle}{\sqrt{2}} .
\end{aligned}
$$

Here $Z_{b}^{\prime}$ is the heavier one, with $M\left(Z_{b}^{\prime}\right)-M\left(Z_{b}\right)=2 \kappa_{b} \simeq 45 \mathrm{MeV}$. This fixes $\kappa_{b}$, which can also be estimated from the mass difference of the charged states in the charm sector $M\left(Z_{c}^{\prime}\right)-M\left(Z_{c}\right)=2 \kappa_{c} \simeq 120$ $\mathrm{MeV}$, and the QCD expectations $\kappa_{b}: \kappa_{c}=m_{c}: m_{b}$. Expressing the states $Z_{b}$ and $Z_{b}^{\prime}$ in the basis of definite $b \bar{b}$ and light quark $q \bar{q}$ spins, it becomes evident that both the $Z_{b}$ and $Z_{b}^{\prime}$ have $s_{b \bar{b}}=1$ and $s_{b \bar{b}}=0$ components,

$$
\left|Z_{b}\right\rangle=\frac{\left|1_{q \bar{q}}, 0_{b \bar{b}}\right\rangle-\left|0_{q \bar{q}}, 1_{b \bar{b}}\right\rangle}{\sqrt{2}}, \quad\left|Z_{b}^{\prime}\right\rangle=\frac{\left|1_{q \bar{q}}, 0_{b \bar{b}}\right\rangle+\left|0_{q \bar{q}}, 1_{b \bar{b}}\right\rangle}{\sqrt{2}} .
$$

Note, that this differs from the corresponding expressions given earlier in Eq. (24), which is based on the molecular interpretation of the states $Z_{b}$ and $Z_{b}^{\prime}$. Defining ( $g$ is the effective couplings at the vertices $\Upsilon Z_{b} \pi$ and $\left.Z_{b} h_{b} \pi\right)$

$$
\begin{aligned}
& g_{Z} \equiv g\left(\Upsilon \rightarrow Z_{b} \pi\right) g\left(Z_{b} \rightarrow h_{b} \pi\right) \propto-\alpha \beta\left\langle h_{b} \mid Z_{b}\right\rangle\left\langle Z_{b} \mid \Upsilon\right\rangle \\
& g_{Z^{\prime}} \equiv g\left(\Upsilon \rightarrow Z_{b}^{\prime} \pi\right) g\left(Z_{b}^{\prime} \rightarrow h_{b} \pi\right) \propto \alpha \beta\left\langle h_{b} \mid Z_{b}^{\prime}\right\rangle\left\langle Z_{b}^{\prime} \mid \Upsilon\right\rangle
\end{aligned}
$$

we note that within errors, Belle data is consistent with the heavy quark spin conservation, which requires $g_{Z}=-g_{Z^{\prime}}$. The two-component nature of the $Z_{b}$ and $Z_{b}^{\prime}$ is also the feature which was pointed out earlier for the $Y_{b}$ in the context of the direct transition $Y_{b}(10890) \rightarrow \Upsilon(1 S) \pi^{+} \pi^{-}$. To determine the coefficients $\alpha$ and $\beta$, one has to resort to $s_{b \bar{b}}: 1 \rightarrow 1$ transitions

$$
\Upsilon(10890) \rightarrow Z_{b} / Z_{b}^{\prime}+\pi \rightarrow \Upsilon(n S) \pi \pi(n=1,2,3) .
$$

The analogous effective couplings are

$$
\begin{aligned}
& f_{Z}=f\left(\Upsilon \rightarrow Z_{b} \pi\right) f\left(Z_{b} \rightarrow \Upsilon(n S) \pi\right) \propto|\beta|^{2}\left\langle\Upsilon(n S) \mid 0_{q \bar{q}}, 1_{b \bar{b}}\right\rangle\left\langle 0_{q \bar{q}}, 1_{b \bar{b}} \mid \Upsilon\right\rangle \\
& f_{Z^{\prime}}=f\left(\Upsilon \rightarrow Z_{b}^{\prime} \pi\right) f\left(Z_{b}^{\prime} \rightarrow \Upsilon(n S) \pi\right) \propto|\alpha|^{2}\left\langle\Upsilon(n S) \mid 0_{q \bar{q}}, 1_{b \bar{b}}\right\rangle\left\langle 0_{q \bar{q}}, 1_{b \bar{b}} \mid \Upsilon\right\rangle .
\end{aligned}
$$


Dalitz analysis indicates that $\Upsilon(10890) \rightarrow Z_{b} / Z_{b}^{\prime}+\pi \rightarrow \Upsilon(n S) \pi \pi(n=1,2,3)$ proceed mainly through the resonances $Z_{b}$ and $Z_{b}^{\prime}$, though $\Upsilon(10890) \rightarrow \Upsilon(1 S) \pi \pi$ has a significant direct component, expected in tetraquark interpretation of $\Upsilon(10890)$ 305. A comprehensive analysis of the Belle data including the direct and resonant components is required to test the underlying dynamics, which is yet to be carried out. However, parametrizing the amplitudes in terms of two Breit-Wigners, one can determine the ratio $\alpha / \beta$ from $\Upsilon(10890) \rightarrow Z_{b} / Z_{b}^{\prime}+\pi \rightarrow \Upsilon(n S) \pi \pi(n=1,2,3)$. For the $s_{b \bar{b}}: 1 \rightarrow 1$ transition, one obtains for the averaged quantities:

$$
\overline{\text { Rel.Norm. }}=0.85 \pm 0.08=|\alpha|^{2} /|\beta|^{2} ; \quad \overline{\text { Rel.Phase }}=(-8 \pm 10)^{\circ}
$$

For the $s_{b \bar{b}}: 1 \rightarrow 0$ transition, the corresponding quantities are

$$
\overline{\text { Rel.Norm. }}=1.4 \pm 0.3 ; \quad \overline{\text { Rel.Phase }}=(185 \pm 42)^{\circ} \text {. }
$$

Within errors, the tetraquark assignment with $\alpha=\beta=1$ is supported, i.e.,

$$
\left|Z_{b}\right\rangle=\frac{\left|1_{b q}, 0_{\bar{b} \bar{q}}\right\rangle-\left|0_{b q}, 1_{\bar{b} \bar{q}}\right\rangle}{\sqrt{2}}, \quad\left|Z_{b}^{\prime}\right\rangle=\left|1_{b q}, 1_{\bar{b} \bar{q}}\right\rangle_{J=1},
$$

and

$$
\left|Z_{b}\right\rangle=\frac{\left|1_{q \bar{q}}, 0_{b \bar{b}}\right\rangle-\left|0_{q \bar{q}}, 1_{b \bar{b}}\right\rangle}{\sqrt{2}}, \quad\left|Z_{b}^{\prime}\right\rangle=\frac{\left|1_{q \bar{q}}, 0_{b \bar{b}}\right\rangle+\left|0_{q \bar{q}}, 1_{b \bar{b}}\right\rangle}{\sqrt{2}} .
$$

It is interesting that similar conclusion was drawn in the 'molecular' interpretation [317] of the $Z_{b}$ and $Z_{b}^{\prime}$.

The Fierz rearrangement used in obtaining second of the above relations would put together the $b \bar{q}$ and $q \bar{b}$ fields, yielding

$$
\left|Z_{b}\right\rangle=\left|1_{b \bar{q}}, 1_{\bar{b} q}\right\rangle_{J=1}, \quad\left|Z_{b}^{\prime}\right\rangle=\frac{\left|1_{b \bar{q}}, 0_{q \bar{b}}\right\rangle+\left|0_{b \bar{q}}, 1_{q \bar{b}}\right\rangle}{\sqrt{2}} .
$$

Here, the labels $0_{b \bar{q}}$ and $1_{\bar{q} b}$ could be viewed as indicating $B$ and $B^{*}$ mesons, respectively, leading to the prediction $Z_{b} \rightarrow B^{*} \bar{B}^{*}$ and $Z_{b}^{\prime} \rightarrow B \bar{B}^{*}$, which is not in agreement with the Belle data [27]. However, this argument rests on the conservation of the light quark spin, for which there is no theoretical foundation. Hence, this last relation is not reliable. Since $Y_{b}(10890)$ and $\Upsilon(5 S)$ are rather close in mass, and there is an issue with the unaccounted direct production of the $B^{*} \bar{B}^{*}$ and $B \bar{B}^{*}$ states in the Belle data collected in their vicinity, we remark that the experimental situation is still in a state of flux and look forward to its resolution with the upcoming Belle-II data.

\subsection{Drell-Yan mechanism for vector exotica production at the LHC and Tevatron}

The exotic hadrons having $J^{\mathrm{PC}}=1^{--}$can be produced at the Tevatron and LHC via the Drell-Yan process [318] $p p(\bar{p}) \rightarrow \gamma^{*} \rightarrow V+\ldots$ The cases $V=\phi(2170), Y(4260), Y_{b}(10890)$ have been studied [318]. With the other two hadrons already discussed earlier, we recall that the $\phi(2170)$ was first observed in the ISR process $e^{+} e^{-} \rightarrow \gamma_{\mathrm{ISR}} f_{0}(980) \phi(1020)$ by BaBar [319] and later confirmed by BESII [320] and Belle 321. Drenska et al. 322 interpreted $\phi(1270)$ as a $P$-wave tetraquark $[s q][\bar{s} \bar{q}]$. Thus, all three vector exotica are assumed to be the first orbital excitation of diquark-antidiquark states with a hidden $s \bar{s}, c \bar{c}$ and $b \bar{b}$ quark content, respectively. Since all three have very small branching ratios in a dilepton pair, they should be searched for in the decay modes in which they have been discovered, all involving 
four charged particles, which, in principle, can be detected in the experiments at hadron colliders. The cross sections for the processes $p \bar{p}(p) \rightarrow \phi(2170)\left(\rightarrow \phi(1020) f_{0}(980) \rightarrow K^{+} K^{-} \pi^{+} \pi^{-}\right), p \bar{p}(p) \rightarrow$ $Y(4260)\left(\rightarrow J / \psi \pi^{+} \pi^{-} \rightarrow \mu^{+} \mu^{-} \pi^{+} \pi^{-}\right)$, and $p \bar{p}(p) \rightarrow Y_{b}(10890)\left(\rightarrow \Upsilon(1 S, 2 S, 3 S) \pi^{+} \pi^{-} \rightarrow \mu^{+} \mu^{-} \pi^{+} \pi^{-}\right)$, at the Tevatron $(\sqrt{s}=1.96 \mathrm{TeV})$ and the LHC are computed in [318]. All these processes have measurable rates, and they should be searched for at the LHC.

Summarizing the tetraquark discussion, we note that there are several puzzles in the $X, Y, Z$ sector. First, and foremost, a very rich spectrum of tertaquark states is predicted in the diquark scenario and the continued absence of many of the predicted states is enigmatic. The nature of the observed states $J^{P C}=1^{--}, Y(4260)$ and $Y(10890)$ is another open question, and whether they are related with each other. Also, whether $Y(10890)$ and $\Upsilon(5 S)$ are one and the same particle is still an open issue. In principle, both $Y(4260)$ and $Y(10890)$ can be produced at the LHC and measured through the $J \psi \pi^{+} \pi^{-}$and $\Upsilon(n S) \pi^{+} \pi^{-}(n S=1 S, 2 S, 3 S)$ modes, respectively. Their hadroproduction crosssections are unfortunately uncertain, but their (normalized) transverse momentum distributions will be quite revealing. As they are both $J^{P C}=1^{--}$hadrons, they can also be produced via the Drell-Yan mechanism and detected through their signature decay modes. The tetraquark interpretation of the charged exotics $Z_{b}$ and $Z_{b}^{\prime}$ leads to a straight forward understanding of the relative rates and strong phases of the heavy quark spin non-flip and spin-flip transitions in the decays $\Upsilon(10890) \rightarrow \Upsilon(n S) \pi^{+} \pi^{-}$ and $\Upsilon(10890) \rightarrow h_{b}(m P) \pi^{+} \pi^{-}$, respectively. However, these transitions can also be accommodated in the hadron molecule approach. In the tetraquark picture, the corresponding hadrons in the charm sector $Z_{c}$ and $Z_{c}^{\prime}$ are related to their $b \bar{b}$ counterparts.

\section{Theoretical models for pentaquarks}

Pentaquarks remained elusive for almost a decade under the shadow of the botched discoveries of $\Theta(1540), \Phi(1860), \Theta_{c}(3100)$. This has definitely changed by the observation of $J / \psi p$ resonances consistent with pentaquark states in $\Lambda_{b}^{0} \rightarrow J / \psi K^{-} p$ decays by the LHCb collaboration [11]. The measured distributions in the invariant masses $m_{K p}$ and $m_{J / \psi p}$ are shown in Fig. 9 together with a model comparison with two $P_{c}^{+}$states. A statistically good fit of the $m_{J / \psi p}$ distribution is consistent with the presence of two resonant states, called $P_{c}(4450)^{+}$and $P_{c}(4380)^{+}$, discussed earlier. Both of these states carry a unit of baryonic number and have the valence quarks $P_{c}^{+}=\bar{c} c u u d$. The preferred $J^{\mathrm{P}}$ assignments are $5 / 2^{+}$for the $P_{c}(4450)^{+}$and $3 / 2^{-}$for the $P_{c}(4380)^{+}$.

The Argand-diagram analysis in the $\left(\operatorname{Im} A^{P_{c}}-\operatorname{Re} A^{P_{c}}\right)$ plane found that the phase change in the amplitude is consistent with a resonance for the $P_{c}(4450)^{+}$, but less so for the $P_{c}(4380)^{+}$, as shown in Fig. 13. The phase diagram for the $P_{c}(4380)^{+}$state needs further study with more data, but the resonant character of the $P_{c}(4450)^{+}$state is very likely. This will be contrasted with the corresponding phase diagram resulting from the assumption that $P_{c}(4450)^{+}$is a kinematically-induced cusp state.

Following a pattern seen for the tetraquark candidates, namely their proximity to respective thresholds, such as $D \bar{D}^{*}$ for the $X(3872), B \bar{B}^{*}$ and $B^{*} \bar{B}^{*}$ for the $Z_{b}(10610)$ and $Z_{b}(10650)$, respectively, also the two pentaquark candidates $P_{c}(4380)$ and $P_{c}(4450)$ lie close to several charm meson-baryon thresholds [323]. The $\Sigma_{c}^{*+} \bar{D}^{0}$ has a threshold of $4382.3 \pm 2.4 \mathrm{MeV}$, tantalizingly close to the mass of $P_{c}(4380)^{+}$. In the case of $P_{c}(4450)^{+}$, there are several thresholds within striking distance, $\chi_{c 1} p(4448.93 \pm$ $0.07), \Lambda_{c}^{*+} \bar{D}^{0}(4457.09 \pm 0.35), \Sigma_{c}^{+} \bar{D}^{* 0}(4459.9 \pm 0.9)$, and $\Sigma_{c}^{+} \bar{D}^{0} \pi^{0}(4452.7 \pm 0.5)$, where the masses are in units of $\mathrm{MeV}$. This has led to a number of hypotheses to explain the two $P_{c}$ states:

- $P_{c}(4380)$ and $P_{c}(4450)$ are baryocharmonia $[114$.

- Rescattering-induced kinematic effects are mimicking the resonances 324 327].

- They are open charm-baryon and charm-meson bound states 328 332. 
- They are compact diquark-diquark-antiquark states [41,333 339, with each component being a $\overline{3}$, yielding a color-singlet $\bar{c}[c q][q q]$ state. Another possibility is via the sequential formation of compact color triplets, making up diquark-triquark systems, yielding also color-singlet states 340 , 341.

In the baryocharmonium picture, the $P_{c}$ states are hadroquarkonium-type composites of $J / \psi$ and excited nucleon states similar to the known resonances $N(1440)$ and $N(1520)$. Photoproduction of the $P_{c}$ states in $\gamma+p$ collisions is advocated as sensitive probe of this mechanism [114. We shall discuss below the interpretation of pentaquarks as scattering-induced kinematic effects, and as meson-baryon molecules, but review the compact diquark-based models in some detail.

\subsection{Pentaquarks as rescattering-induced kinematic effects}

Kinematic effects can result in a narrow structure around the $\chi_{c 1} p$ threshold. Two possible mechanisms shown in Fig. 50 are: (a) 2-point loop with a 3-body production $\Lambda_{b}^{0} \rightarrow K^{-} \chi_{c 1} p$ followed by the rescattering process $\chi_{c 1} p \rightarrow J / \psi p$, and (b) in which $K^{-} p$ is produced from an intermediate $\Lambda^{*}$ and the proton rescatters with the $\chi_{c 1}$ into a $J / \psi p$, as shown below.

The amplitude for Fig. 50(a) can be expressed as

$$
G_{\Lambda}(E)=\int \frac{d^{3} q}{(2 \pi)^{3}} \frac{\vec{q}^{2} f_{\Lambda}\left(\vec{q}^{2}\right)}{E-m_{p}-m_{\chi_{c 1}}-\vec{q}^{2} /(2 \mu)},
$$

where $\mu$ is the reduced mass and $f_{\Lambda}\left(\vec{q}^{2}\right)=\exp \left(-2 \vec{q}^{2} / \Lambda^{2}\right)$ is a form factor to regularize the loop integral. Fitting the Argand diagram for the $P_{c}(4450)^{+}$with $\mathcal{A}_{(a)}=N\left(b+G_{\Lambda}(E)\right)$ determines the normalization $N$, the constant background $b$, and $\Lambda$. The integral can be solved analytically [324]

$$
G_{\Lambda}(E)=\frac{\mu \Lambda}{(2 \pi)^{3 / 2}}\left(k^{2}+\Lambda^{2} / 4\right)+\frac{\mu k^{3}}{2 \pi} \exp ^{-2 k^{2} / \Lambda^{2}}\left[\operatorname{erfc}\left(\frac{\sqrt{2} k}{\Lambda}\right)-i\right]
$$

where $k=\sqrt{2 \mu\left(E-m_{1}-m_{2}+i \epsilon\right)}$. This function has a characteristic phase motion reflecting the error function (erfc), as shown in Fig. 51. It differs from the Breit-Wigner fit, which is in excellent agreement with the LHCb data [11]. The cusp-based fit also shows a counter-clockwise behavior in the Argand diagram, but not for the two data points where the imaginary part of the cusp amplitude is zero. The absolute value of the amplitude in the cusp approach shows a resonant behavior, which can be made to peak even more sharply at $\operatorname{Re} \sqrt{s}=4450 \mathrm{MeV}$, if the amplitude for Fig. 50 (b) is included and assumed dominated by the $\Lambda^{*}(1890)$-exchange. However, it is the phase motion, which is decisive in distinguishing a dynamical Breit-Wigner (or, for that matter a Flatte [97] type) resonance and a kinematic-induced cusp behavior. More data is needed to completely settle this difference in the case of $P_{c}(4450)^{+}$, but currently the Breit-Wigner fit is the preferred description.

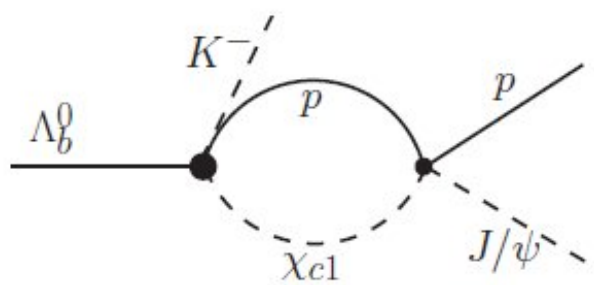

(a)

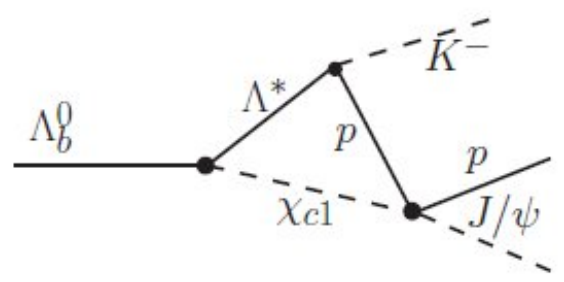

(b)

Figure 50: The two scattering diagrams discussed in the text( from 324]). 


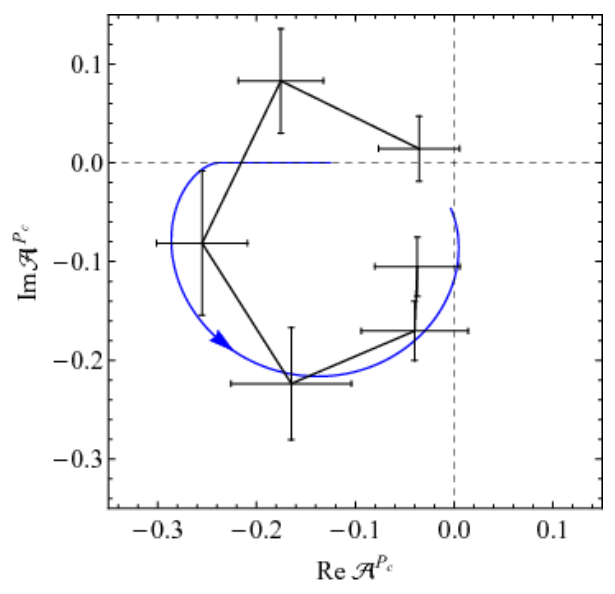

Figure 51: Fitted values of the real and imaginary parts of the amplitudes for the $P_{c}(4450)^{+}$using a Breit-Wigner formula with $M(\Gamma)$ of $4450(39) \mathrm{MeV}$ [11]. The directed curve (blue) is the fit in the cusp model. (from 324]).

\subsection{Pentaquarks as meson-baryon molecules}

In the hadronic molecular interpretation, one identifies the $P_{c}(4380)^{+}$with $\Sigma_{c}(2455) \bar{D}^{*}$ and the $P_{c}(4450)^{+}$ with $\Sigma_{c}(2520) \bar{D}^{*}$, which are bound by a pion exchange. The underlying interaction can be expressed in terms of the effective Lagrangians [331]:

$$
\begin{aligned}
& \mathcal{L}_{\mathcal{P}}=i g \operatorname{Tr}\left[\bar{H}_{a}^{(\bar{Q})} \gamma^{\mu} A_{a b}^{\mu} \gamma_{5} H_{b}^{(\bar{Q})}\right] \\
& \mathcal{L}_{\mathcal{S}}=-\frac{3}{2} g_{1} \epsilon^{\mu \lambda \nu \kappa} v_{\kappa} \operatorname{Tr}\left[\overline{\mathcal{S}}_{\mu} A_{\nu} \mathcal{S}_{\lambda}\right]
\end{aligned}
$$

which are built using the heavy quark and chiral symmetries. Here $H_{a}^{(\bar{Q})}=\left[P_{a}^{*(\bar{Q}) \mu} \gamma_{\mu}-P_{a}^{(\bar{Q})} \gamma_{5}\right](1-\psi) / 2$ is a pseudoscalar and vector charmed meson multiplet $\left(D, D^{*}\right), v$ being the four-velocity vector $v=(0, \overrightarrow{1})$, $\mathcal{S}_{\mu}=1 / \sqrt{3}\left(\gamma_{\mu}+v_{\mu}\right) \gamma^{5} \mathcal{B}_{6}+\mathcal{B}_{6 \mu}^{*}$ stands for the charmed baryon multiplet, with $\mathcal{B}_{6}$ and $\mathcal{B}_{6 \mu}^{*}$ corresponding to the $J^{P}=1 / 2^{+}$and $J^{P}=3 / 2^{+}$in $6_{F}$ flavor representation, respectively. $A_{\mu}$ is an axial-vector current, containing a pion chiral multiplet, defined as $A_{\mu}=1 / 2\left(\xi^{\dagger} \partial_{\mu} \xi-\xi \partial_{\mu} \xi^{\dagger}\right)$, with $\xi=\exp \left(i \mathbb{P} / f_{\pi}\right)$, with $\mathbb{P}$ an $S U(2)$ matrix containing the pion field, and $f_{\pi}=132 \mathrm{MeV}$. This interaction Lagrangian is used to work out effective potentials, energy levels and wave-functions of the $\Sigma_{c}^{(*)} \bar{D}^{*}$ systems, shown in Fig. 52 . In this picture, $P_{c}(4380)^{+}$is a $\Sigma_{c} \bar{D}^{*}(I=1 / 2, J=3 / 2)$ molecule, and $P_{c}(4450)^{+}$is a $\Sigma_{c}^{*} \bar{D}^{*}(I=1 / 2, J=5 / 2)$ molecule.

Apart from accommodating the two observed pentaquarks, this framework predicts two additional hidden-charm molecular pentaquark states, $\Sigma_{c} \bar{D}^{*}(I=3 / 2, J=1 / 2)$ and $\Sigma_{c}^{*} \bar{D}^{*}(I=3 / 2, J=1 / 2)$, which are isospin partners of $P_{c}(4380)^{+}$and $P_{c}(4450)^{+}$, respectively, decaying into $\Delta(1232) \mathrm{J} / \psi$ and $\Delta(1232) \eta_{c}$. In addition, a rich pentaquark spectrum of states for the hidden-bottom $\left(\Sigma_{b} B^{*}, \Sigma_{b}^{*} B^{*}\right)$, $B_{c^{-}}$like $\left(\Sigma_{c} B^{*}, \Sigma_{c}^{*} B^{*}\right)$ and $\left(\Sigma_{b} \bar{D}^{*}, \Sigma_{b}^{*} \bar{D}^{*}\right)$ with well-defined $(I, J)$ is predicted.

\subsection{Pentaquarks in the compact diquark models}

In the paper by Maiani et al. [41] on the pentaquark interpretation of the LHCb data on $\Lambda_{b}^{0} \rightarrow J / \psi p K^{-}$ decay, which is mainly discussed here, the assigned internal quantum numbers are: $P_{c}^{+}(4450)=$ $\left\{\bar{c}[c u]_{s=1}[u d]_{s=0} ; L_{\mathcal{P}}=1, J^{\mathrm{P}}=\frac{5}{2}^{+}\right\}$and $P_{c}^{+}(4380)=\left\{\bar{c}[c u]_{s=1}[u d]_{s=1} ; L_{\mathcal{P}}=0, J^{\mathrm{P}}=\frac{3}{2}^{-}\right\}$. Taking into account the mass differences due to the orbital angular momentum and the light diquark spins, the observed mass difference between the two $P_{c}^{+}$states of about $70 \mathrm{MeV}$ is approximately reproduced. The crucial assumption is that the two diagrams for the decay $\Lambda_{b}^{0} \rightarrow J / \psi p K^{-}$in which the $u d$-spin in 
$\Lambda_{b}^{0}$ goes over to the $[u d]$-diquark spin in the pentaquark, Fig. 53(A), and the one in which the $u d$-spin is shared among the final state pentaquark and a meson, generating a light diquark [ud] having spin-0 and spin-1, Fig. 53(B), are treated at par. This is a dynamical assumption, and remains to be tested.

\section{4 $S U(3)_{F}$ structure of pentaquarks}

Concentrating on the quark flavor of the pentaquarks $\mathbb{P}_{c}^{+}=\bar{c} c u u d$, they are of two different types [41]:

$$
\begin{aligned}
\mathbb{P}_{u} & =\epsilon^{\alpha \beta \gamma} \bar{c}_{\alpha}[c u]_{\beta, s=0,1}[u d]_{\gamma, s=0,1}, \\
\mathbb{P}_{d} & =\epsilon^{\alpha \beta \gamma} \bar{c}_{\alpha}[c d]_{\beta, s=0,1}[u u]_{\gamma, s=1},
\end{aligned}
$$

the difference being that the $\mathbb{P}_{d}$ involves a $[u u]$ diquark, and the Pauli exclusion principle implies that this diquark has to be in an $S U(3)_{F}$-symmetric representation. This leads to two distinct $S U(3)_{F}$ series of pentaquarks

$$
\begin{aligned}
& \mathbb{P}_{A}=\epsilon^{\alpha \beta \gamma}\left\{\bar{c}_{\alpha}[c q]_{\beta, s=0,1}\left[q^{\prime} q^{\prime \prime}\right]_{\gamma, s=0}, L\right\}=\mathbf{3} \otimes \overline{\mathbf{3}}=\mathbf{1} \oplus \mathbf{8}, \\
& \mathbb{P}_{S}=\epsilon^{\alpha \beta \gamma}\left\{\bar{c}_{\alpha}[c q]_{\beta, s=0,1}\left[q^{\prime} q^{\prime \prime}\right]_{\gamma, s=1}, L\right\}=\mathbf{3} \otimes \mathbf{6}=\mathbf{8} \oplus \mathbf{1 0} .
\end{aligned}
$$

For $S$ waves, the first and the second series have the angular momenta

$$
\begin{aligned}
\mathbb{P}_{A}(L=0): & J=1 / 2(2), 3 / 2(1), \\
\mathbb{P}_{S}(L=0): & J=1 / 2(3), 3 / 2(3), 5 / 2(1),
\end{aligned}
$$

where the multiplicities are given in parentheses. One assigns $\mathbb{P}\left(3 / 2^{-}\right)$to the $\mathbb{P}_{A}$ and $\mathbb{P}\left(5 / 2^{+}\right)$to the $\mathbb{P}_{S}$ series of pentaquarks 41$]$.

The decay amplitudes of interest of a $b$-baryon $\mathcal{B}$ to an octet of pseudoscalar meson $\mathcal{M}$ and a pentaquark with a hidden $c \bar{c}, \mathcal{P}$, can be generically written as

$$
\mathcal{A}=\left\langle\mathcal{P} \mathcal{M}\left|H_{\mathrm{eff}}^{W}\right| \mathcal{B}\right\rangle
$$

where, $H_{\mathrm{eff}}^{W}$ is the effective weak Hamiltonian inducing the Cabibbo-allowed $\Delta I=0, \Delta S=-1$ transition $b \rightarrow c \bar{c} s$, and the Cabibbo-suppressed $\Delta S=0$ transition $b \rightarrow c \bar{c} d$. The $S U(3)_{F}$ based analysis of the
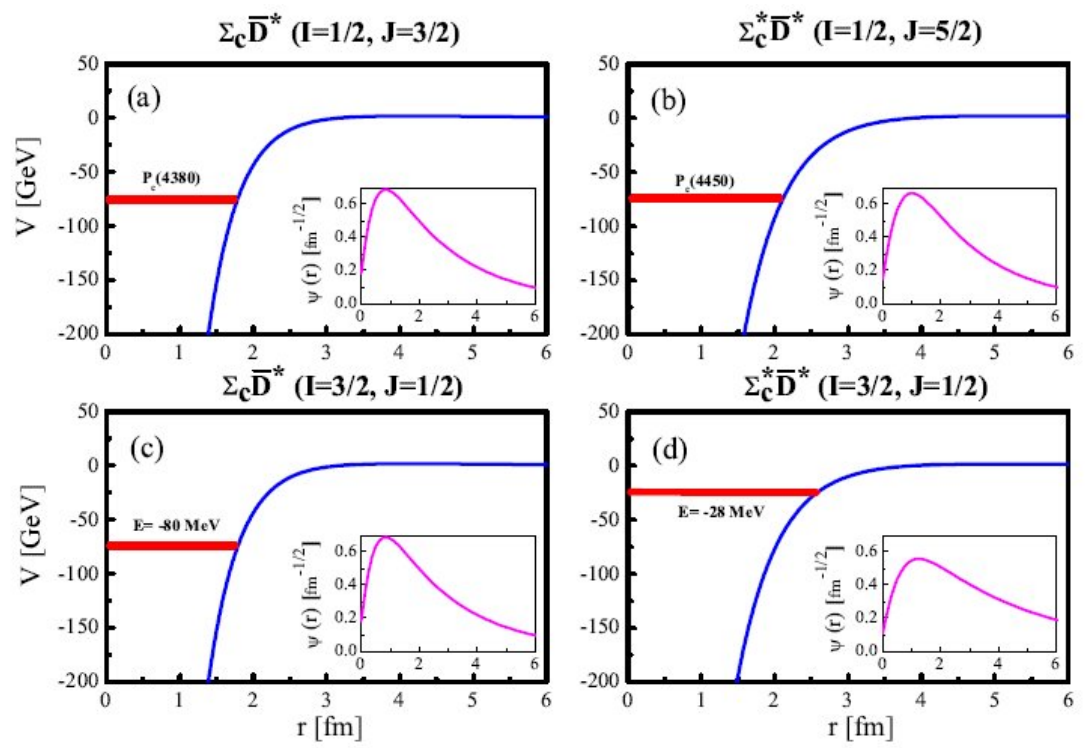

Figure 52: Effective potentials, $V(\mathrm{GeV})$, energy levels, thick (red) lines, and wave-functions, $\psi(r)$, of the $\Sigma_{c}^{(*)} \bar{D}^{*}$ system (from 331 ). 
decays $\Lambda_{b} \rightarrow \mathbb{P}^{+} K^{-} \rightarrow(J / \psi p) K^{-}$goes as follows. With respect to $S U(3)_{F}, \Lambda_{b}(b u d) \sim \overline{3}$ and it is an isosinglet $I=0$. Thus, the weak non-leptonic Hamiltonian for $b \rightarrow c \bar{c} q(q=s, d)$ decays is:

$$
H_{\mathrm{eff}}^{W}=\frac{4 G_{F}}{\sqrt{2}}\left[V_{c b} V_{c q}^{*}\left(c_{1} O_{1}^{(q)}+c_{2} O_{2}^{(q)}\right)\right] .
$$

Here, $G_{F}$ is the Fermi coupling constant, $V_{i j}$ are the CKM matrix elements, and $c_{i}$ are the Wilson coefficients of the operators $O_{1}^{(q)}(q=d, s)$, defined as

$$
O_{1}^{(q)}=\left(\bar{q}_{\alpha} c_{\beta}\right)_{V-A}\left(\bar{c}_{\alpha} b_{\beta}\right)_{V-A} ; \quad O_{2}^{(q)}=\left(\bar{q}_{\alpha} c_{\alpha}\right)_{V-A}\left(\bar{c}_{\beta} b_{\beta}\right)_{V-A},
$$

where $\alpha$ and $\beta$ are $S U(3)$ color indices, and $V-A=1-\gamma_{5}$ reflects that the charged currents are lefthanded, and the penguin amplitudes are ignored. With $M$ a nonet of $S U(3)$ light mesons $\left(\pi, K, \eta, \eta^{\prime}\right)$, the weak transitions $\left\langle\mathbb{P}, M\left|H_{\mathrm{W}}\right| \Lambda_{b}\right\rangle$ requires $\mathbb{P}+M$ to be in $8 \oplus 1$ representation. Recalling the $S U(3)$ group multiplication rules

$$
\begin{aligned}
& 8 \otimes 8=1 \oplus 8 \oplus 8 \oplus 10 \oplus \overline{10} \oplus 27, \\
& 8 \otimes 10=8 \oplus 10 \oplus 27 \oplus 35,
\end{aligned}
$$

the decay $\left\langle\mathbb{P}, M\left|H_{\mathrm{W}}\right| \Lambda_{b}\right\rangle$ can be realized with $\mathbb{P}$ in either an octet 8 or a decuplet 10 . The discovery channel $\Lambda_{b} \rightarrow \mathbb{P}^{+} K^{-} \rightarrow J / \psi p K^{-}$corresponds to $\mathbb{P}$ in an octet 8.

\subsection{An effective Hamiltonian for the hidden charm pentaquarks}

Keeping the basic building blocks of the pentaquarks to be quarks and diquarks, we follow here the template in which the two $P_{c}$ states are assumed to be made from five quarks, consisting of two highly correlated diquark pairs, and an antiquark. For the present discussion, it is an anti-charm quark $\bar{c}$ which is correlated with the two diquarks $[c q]$ and $\left[q^{\prime} q^{\prime \prime}\right]$, where $q, q^{\prime}, q^{\prime \prime}$ can be $u$ or $d$. The tetraquark formed by the diquark-diquark $\left([c q]_{\overline{3}}\left[q^{\prime} q^{\prime \prime}\right]_{\overline{3}}\right)$ is a color-triplet object, following from $\overline{3} \times \overline{3}=\overline{6}+3$, with orbital and spin quantum numbers, denoted by $L_{\mathcal{Q Q}}$ and $S_{\mathcal{Q Q}}$, which combines with the color-anti-triplet $\overline{3}$ of the $\bar{c}$ to form an overall color-singlet pentaquark, with the corresponding quantum numbers $L_{\mathcal{P}}$ and $S_{\mathcal{P}}$. This is shown schematically in Fig. 54 .

An effective Hamiltonian based on this picture is constructed [339], extending the underlying tetraquark Hamiltonian developed for the $X, Y, Z$ states [39]. It involves the constituent diquarks masses, $m_{[c q]}, m_{\left[q^{\prime} q^{\prime \prime}\right]}$, the spin-spin interactions between the quarks in each diquark shell, and the spinorbit and orbital angular momentum of the tetraquarks. To this are added the charm quark mass $m_{c}$, the spin-orbit and the orbital terms of the pentaquarks.
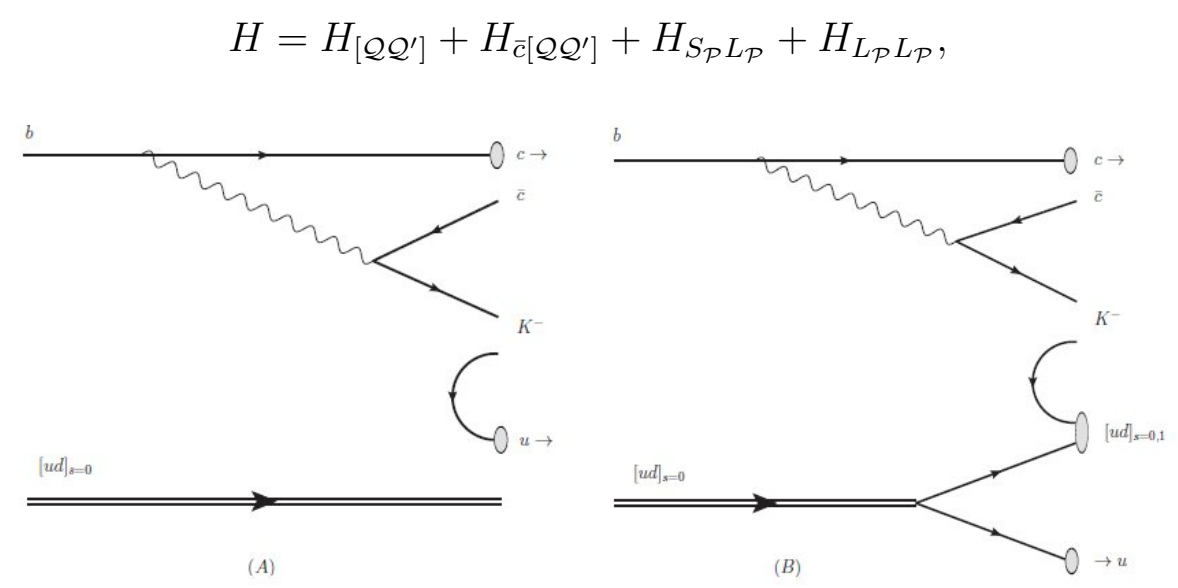

Figure 53: Two mechanisms for the decays $\Lambda_{b}^{0} \rightarrow J / \psi K^{-} p$ in the diquark picture (from [41]). 


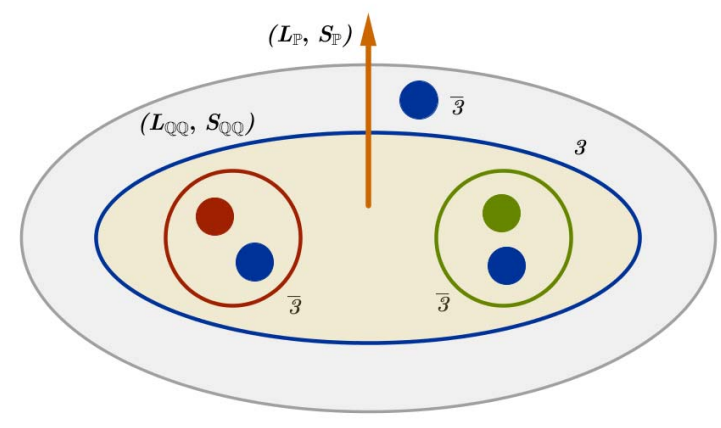

Diquark - Diquark - Antiquark Model of Pentaquarks

Figure 54: $S U(3)$-color quantum numbers of the diquarks, tetraquark and antiquark are indicated, together with the orbital and spin quantum numbers of the tetraquark and pentaquark (from [339]).

where the diquarks $[c q]$ and $\left[q^{\prime} q^{\prime \prime}\right]$ are denoted by $\mathcal{Q}$ and $\mathcal{Q}^{\prime}$ having masses $m_{\mathcal{Q}}$ and $m_{\mathcal{Q}^{\prime}}$, respectively. $L_{\mathcal{P}}$ and $S_{\mathcal{P}}$ are the orbital angular momentum and the spin of the pentaquark state, and the quantities $A_{\mathcal{P}}$ and $B_{\mathcal{P}}$ parametrize the strength of their spin-orbit and orbital angular momentum couplings, respectively. The individual terms in the Hamiltonian (53) are given in [339].

The mass formula for the pentaquark state with the ground state tetraquark $\left(L_{\mathcal{Q} \mathcal{Q}^{\prime}}=0\right)$ can be written as

$$
M=M_{0}+\frac{B_{\mathcal{P}}}{2} L_{\mathcal{P}}\left(L_{\mathcal{P}}+1\right)+2 A_{\mathcal{P}} \frac{J_{\mathcal{P}}\left(J_{\mathcal{P}}+1\right)-L_{\mathcal{P}}\left(L_{\mathcal{P}}+1\right)-S_{\mathcal{P}}\left(S_{\mathcal{P}}+1\right)}{2}+\Delta M
$$

where $M_{0}=m_{\mathcal{Q}}+m_{\mathcal{Q}^{\prime}}+m_{c}$ and $\Delta M$ is the mass term that arises from different spin-spin interactions. With the tetraquark in $L_{\mathcal{Q} \mathcal{Q}^{\prime}}=1$, one has to add the two terms given above with their coefficients $A_{\mathcal{Q} \mathcal{Q}^{\prime}}$ and $B_{\mathcal{Q} \mathcal{Q}^{\prime}}$. In this work, we restrict ourselves to the $S$-wave tetraquarks.

For $L_{\mathcal{P}}=0$, the pentaquark states are classified in terms of the diquarks spins, $S_{\mathcal{Q}}$ and $S_{\mathcal{Q}^{\prime}}$; the spin of anti-charm quark is $S_{\bar{c}}=1 / 2$. There are four $S$-wave pentaquark states for $J^{P}=\frac{3}{2}^{-}$and a single state with $J^{P}=\frac{5}{2}^{-}$. For $J^{P}=\frac{3}{2}^{-}$, we have the following states 16 ,

$$
\begin{aligned}
\left|0_{\mathcal{Q}}, 1_{\mathcal{Q}^{\prime}}, \frac{1}{2} ; \frac{3}{2}\right\rangle_{1}= & \frac{1}{\sqrt{2}}\left[(\uparrow)_{c}(\downarrow)_{q}-(\downarrow)_{c}(\uparrow)_{q}\right](\uparrow)_{q^{\prime}}(\uparrow)_{q^{\prime \prime}}(\uparrow)_{\bar{c}} \\
\left|1_{\mathcal{Q}}, 0_{\mathcal{Q}^{\prime}}, \frac{1}{2} ; \frac{3}{2}\right\rangle_{2}= & \frac{1}{\sqrt{2}}\left[(\uparrow)_{q^{\prime}}(\downarrow)_{q^{\prime \prime}}-(\downarrow)_{q^{\prime}}(\uparrow)_{q^{\prime \prime}}\right](\uparrow)_{c}(\uparrow)_{q}(\uparrow)_{\bar{c}} \\
\left|1_{\mathcal{Q}}, 1_{\mathcal{Q}^{\prime}}, \frac{1}{2} ; \frac{3}{2}\right\rangle_{3}= & \frac{1}{\sqrt{6}}(\uparrow)_{c}(\uparrow)_{q}\left\{2(\uparrow)_{q^{\prime}}(\uparrow)_{q^{\prime \prime}}(\downarrow)_{\bar{c}}-\left[(\uparrow)_{q^{\prime}}(\downarrow)_{q^{\prime \prime}}+(\downarrow)_{q^{\prime}}(\uparrow)_{q^{\prime \prime}}\right](\uparrow)_{\bar{c}}\right\} \\
\left|1_{\mathcal{Q}}, 1_{\mathcal{Q}^{\prime}}, \frac{1}{2} ; \frac{3}{2}\right\rangle_{4}= & \sqrt{\frac{3}{10}}\left[(\uparrow)_{c}(\downarrow)_{q}+(\downarrow)_{c}(\uparrow)_{q}\right](\uparrow)_{q^{\prime}}(\uparrow)_{q^{\prime \prime}}(\uparrow)_{\bar{c}}-\sqrt{\frac{2}{15}}(\uparrow)_{c}(\uparrow)_{q}\left\{(\uparrow)_{q^{\prime}}(\uparrow)_{q^{\prime \prime}}(\downarrow)_{\bar{c}}\right. \\
& \left.+\left[(\uparrow)_{q^{\prime}}(\downarrow)_{q^{\prime \prime}}+(\downarrow)_{q^{\prime}}(\uparrow)_{q^{\prime \prime}}\right](\uparrow)_{\bar{c}}\right\},
\end{aligned}
$$

and the spin representation corresponding to $J^{P}=\frac{5}{2}^{-}$state is:

$$
\left|1_{\mathcal{Q}}, 1_{\mathcal{Q}^{\prime}}, \frac{1}{2} ; \frac{5}{2}\right\rangle=(\uparrow)_{c}(\uparrow)_{q}(\uparrow)_{q^{\prime}}(\uparrow)_{q^{\prime \prime}}(\uparrow)_{\bar{c}}
$$

The masses for the four $S$-wave pentaquark states with $J^{P}=\frac{3}{2}^{-}$and a single state with $J^{P}=\frac{5}{2}^{-}$in terms of the parameters of the effective Hamiltonian are given in Table 22, where we label the states as $\mathcal{P}_{X_{i}}$. The corresponding five $P$-wave pentaquark states with $L_{\mathcal{P}}=1$ and $J^{P}=\frac{5}{2}^{+}$are labeled as $\mathcal{P}_{Y_{i}}$ in Table 22, $\Delta M_{i}$ are defined in [339], where also the various input parameters are given. The resulting

\footnotetext{
${ }^{16}$ For a similar classification in the diquark-triquark picture, see 341 .
} 
Table 22: $S(P)$ - wave pentaquark states $\mathcal{P}_{X_{i}}\left(\mathcal{P}_{Y_{i}}\right)$ and their spin- and orbital angular momentum quantum numbers. The subscripts $\mathcal{Q}$ and $\mathcal{Q}^{\prime}$ represent the heavy $[c q]$ and light $\left[q^{\prime} q^{\prime \prime}\right]$ diquarks, respectively. In the expressions for the masses of the $\mathcal{P}_{Y_{i}}$ states, the terms $M_{\mathcal{P}_{X_{i}}}=M_{0}+\Delta M_{i}$ with $i=1, \ldots, 5$.

\begin{tabular}{llllll}
\hline Label & $\left|S_{\mathcal{Q}}, S_{\mathcal{Q}^{\prime}} ; L_{\mathcal{P}}, J^{P}\right\rangle_{i}$ & Mass & Label & $\left|S_{\mathcal{Q}}, S_{\mathcal{Q}^{\prime}} ; L_{\mathcal{P}}, J^{P}\right\rangle_{i}$ & Mass \\
\hline $\mathcal{P}_{X_{1}}$ & $\left|0_{\mathcal{Q}}, 1_{\mathcal{Q}^{\prime}}, 0 ; \frac{3}{2}{ }^{-}\right\rangle_{1}$ & $M_{0}+\Delta M_{1}$ & $\mathcal{P}_{Y_{1}}$ & $\left|0_{\mathcal{Q}}, 1_{\mathcal{Q}^{\prime}}, 1 ; \frac{5}{2}^{+}\right\rangle_{1}$ & $M_{\mathcal{P}_{X_{1}}}+3 A_{\mathcal{P}}+B_{\mathcal{P}}$ \\
\hline $\mathcal{P}_{X_{2}}$ & $\left|1_{\mathcal{Q}}, 0_{\mathcal{Q}^{\prime}}, 0 ; \frac{3}{2}^{-}\right\rangle_{2}$ & $M_{0}+\Delta M_{2}$ & $\mathcal{P}_{Y_{2}}$ & $\left|1_{\mathcal{Q}}, 0_{\mathcal{Q}^{\prime}}, 1 ; \frac{5}{2}{ }^{+}\right\rangle_{2}$ & $M_{\mathcal{P}_{X_{2}}}+3 A_{\mathcal{P}}+B_{\mathcal{P}}$ \\
\hline $\mathcal{P}_{X_{3}}$ & $\left|1_{\mathcal{Q}}, 1_{\mathcal{Q}^{\prime}}, 0 ; \frac{3}{2}{ }^{-}\right\rangle_{3}$ & $M_{0}+\Delta M_{3}$ & $\mathcal{P}_{Y_{3}}$ & $\left|1_{\mathcal{Q}}, 1_{\mathcal{Q}^{\prime}}, 1 ; \frac{5}{2}^{+}\right\rangle_{3}$ & $M_{\mathcal{P}_{X_{3}}}+3 A_{\mathcal{P}}+B_{\mathcal{P}}$ \\
\hline $\mathcal{P}_{X_{4}}$ & $\left|1_{\mathcal{Q}}, 1_{\mathcal{Q}^{\prime}}, 0 ; \frac{3}{2}^{-}\right\rangle_{4}$ & $M_{0}+\Delta M_{4}$ & $\mathcal{P}_{Y_{4}}$ & $\left|1_{\mathcal{Q}}, 1_{\mathcal{Q}^{\prime}}, 1 ; \frac{5}{2}{ }^{+}\right\rangle_{4}$ & $M_{\mathcal{P}_{X_{4}}}+3 A_{\mathcal{P}}+B_{\mathcal{P}}$ \\
\hline $\mathcal{P}_{X_{5}}$ & $\left|1_{\mathcal{Q}}, 1_{\mathcal{Q}^{\prime}}, 0 ; \frac{5}{2}^{-}\right\rangle_{5}$ & $M_{0}+\Delta M_{5}$ & $\mathcal{P}_{Y_{5}}$ & $\left|1_{\mathcal{Q}}, 1_{\mathcal{Q}^{\prime}}, \frac{1}{2} \bar{c}, 1 ; \frac{5}{2}{ }^{+}\right\rangle_{5}$ & $M_{\mathcal{P}_{X_{5}}}-2 A_{\mathcal{P}}+B_{\mathcal{P}}$ \\
\hline
\end{tabular}

mass spectrum of the $S$ - and $P$-wave pentaquarks, with $J^{P}=\left(3 / 2^{-}, 5 / 2^{-}\right)$and $J^{P}=5 / 2^{+}$, respectively, and having the quark flavor content $\bar{c}[c q][q q], \bar{c}[c q][s q], \bar{c}[c s][q q], \bar{c}[c s][s q]$, and $\bar{c}[c q][s s]$, is given in Table 23. Later, for ease of writing, the labels $c_{1}, \ldots, c_{5}$ will be used for these quark flavor combinations. Thus, for each of the $c_{i}$, the masses of the $S(P)$-wave pentaquark states $X_{j}\left(Y_{j}\right), j=1, \ldots, 5$ can be read off from this table. In working out the masses, isospin-symmetry is used in that the small $m_{d}-m_{u}$ mass difference is ignored. Thus, the pentaquark states with the quark content $\bar{c}[c u][u d]$ and $\bar{c}[c d][u d]$ are mass degenerate. These states will be denoted subsequently by a subscript $P_{p}$ and $P_{n}$, respectively, and the notation is such that the light-quark content of the pentaquark is represented by the corresponding light baryon.

To make the notation clear, let us consider the decay $\Lambda_{b}^{0} \rightarrow P_{c}(4450)^{+}(\rightarrow J / \psi p) K^{-}$. In our notation, this decay is expressed as $\Lambda_{b}^{0} \rightarrow P_{p}^{\left\{Y_{2}\right\}_{c_{1}}}(\rightarrow J / \psi p) K^{-}$, with the diquark-spin and angular momentum quantum numbers given by the entry $\mathcal{P}_{Y_{2}}$ in Table 22 , and its mass is given by the entry for $c_{1}=\bar{c}[c q][q q](4450 \pm 57 \mathrm{MeV})$ in Table 23. The isospin-related decay (which is not yet seen) in our notation is $\Lambda_{b}^{0} \rightarrow P_{n}^{\left\{Y_{2}\right\}_{c_{1}}}(\rightarrow J / \psi n) \bar{K}^{0}$, where the pentaquark $P_{n}^{\left\{Y_{2}\right\}_{c_{1}}}$ is the neutral partner of $P_{c}(4450)^{+}$, having $J^{P}=5 / 2^{+}$, and degenerate in mass. By isospin, their decay rates are also the same. The masses of the entire $S U(3)_{F}$ multiplets of pentaquarks with the given $J^{P}$ quantum numbers and their decays are worked out using this notation. In addition to these, also the spectroscopy of the pentaquarks having $J^{P}=1 / 2^{ \pm}$and the quark flavor content as shown in Table 23 have been worked out in the compact diquark picture. However, as none of these states have so far been discovered, we restrict the discussion to the $J^{P}=\left(3 / 2^{-}, 5 / 2^{-}\right)$and $J^{P}=5 / 2^{+}$pentaquarks, since two such candidates have been observed by the LHCb. These tables illustrate that the spectrum of pentaquark states in the compact diquark model is very rich. Apart from the other predicted states, there is a state, $\mathcal{P}_{X_{4}}$, which is predicted to have a mass around $4385 \mathrm{MeV}$, having the quantum numbers $\left|1_{\mathcal{Q}}, 1_{\mathcal{Q}^{\prime}}, 0 ; \frac{3}{2}^{-}\right\rangle$. This agrees with the mass of the observed state $P_{c}^{+}(4380)$. Likewise, the state $P_{c}^{+}(4550)$, having $J^{P}=\frac{5}{2}^{+}$can be identified with the state $\mathcal{P}_{Y_{2}}$ in the first row of Table 22 , having the quantum numbers $\left|1_{\mathcal{Q}}, 0_{\mathcal{Q}^{\prime}}, 1 ; \frac{5}{2}{ }^{+}\right\rangle$. We recall that these two states have the same internal quantum numbers as in Ref. Maiani et al. [41]:

$$
\begin{aligned}
& P_{c}(4380)^{+}=\mathbb{P}^{+}\left(3 / 2^{-}\right)=\left\{\bar{c}[c q]_{s=1}\left[q^{\prime} q^{\prime \prime}\right]_{s=1}, L=0\right\} \\
& P_{c}(4450)^{+}=\mathbb{P}^{+}\left(5 / 2^{+}\right)=\left\{\bar{c}[c q]_{s=1}\left[q^{\prime} q^{\prime \prime}\right]_{s=0}, L=1\right\} .
\end{aligned}
$$

\section{6 b-baryon decays to pentaquarks and heavy quark symmetry}

The pentaquark states reported by the LHCb are produced in $\Lambda_{b}^{0}$ decays, $\Lambda_{b}^{0} \rightarrow \mathcal{P}^{+} K^{-}$, where $\mathcal{P}$ denotes a generic pentaquark state. QCD has a symmetry in the heavy quark limit, i.e., for $m_{b} \gg \Lambda_{\mathrm{QCD}}, b$-quark becomes a static quark and the light diquark spin becomes a good quantum number, constraining the states which can otherwise be produced. The $b$-baryon decays to pentaquarks having a $c \bar{c}$ component 
Table 23: Masses of the $S$ - and $P$-wave pentaquarks, $\mathcal{P}_{X_{i}}$ and $\mathcal{P}_{Y_{i}}$ and having the $J^{P}$ quantum numbers given in Table 22, (in MeV) formed through different diquark-diquark-anti-charm quark combinations in type-I diquark model. The quoted errors are obtained from the uncertainties in the input parameters in the effective Hamiltonian. The light-quark content is given explicitly (with $q=u$ or $d$ ) (From [339]).

\begin{tabular}{llllll}
\hline $\mathcal{P}_{X_{i}}$ & $\mathcal{P}_{X_{1}}$ & $\mathcal{P}_{X_{2}}$ & $\mathcal{P}_{X_{3}}$ & $\mathcal{P}_{X_{4}}$ & $\mathcal{P}_{X_{5}}$ \\
\hline $\bar{c}[c q][q q]$ & $4133 \pm 55$ & $4133 \pm 55$ & $4197 \pm 55$ & $4385 \pm 55$ & $4534 \pm 55$ \\
$\bar{c}[c q][s q]$ & $4115 \pm 58$ & $4138 \pm 47$ & $4191 \pm 53$ & $4324 \pm 47$ & $4478 \pm 47$ \\
$\bar{c}[c s][q q]$ & $4365 \pm 55$ & $4390 \pm 42$ & $4443 \pm 49$ & $4578 \pm 43$ & $4727 \pm 42$ \\
$\bar{c}[c s][s q]$ & $4313 \pm 47$ & $4382 \pm 45$ & $4434 \pm 51$ & $4568 \pm 46$ & $4721 \pm 45$ \\
$\bar{c}[c q][s s]$ & $4596 \pm 47$ & $4664 \pm 46$ & $4721 \pm 51$ & $4853 \pm 46$ & $5006 \pm 45$ \\
\hline $\mathcal{P}_{Y_{i}}$ & $\mathcal{P}_{Y_{1}}$ & $\mathcal{P}_{Y_{2}}$ & $\mathcal{P}_{Y_{3}}$ & $\mathcal{P}_{Y_{4}}$ & $\mathcal{P}_{Y_{5}}$ \\
\hline $\bar{c}[c q][q q]$ & $4450 \pm 57$ & $4450 \pm 57$ & $4515 \pm 57$ & $4702 \pm 58$ & $4589 \pm 56$ \\
$\bar{c}[c q][s q]$ & $4432 \pm 61$ & $4456 \pm 50$ & $4508 \pm 56$ & $4642 \pm 50$ & $4532 \pm 48$ \\
$\bar{c}[c s][q q]$ & $4682 \pm 57$ & $4708 \pm 46$ & $4760 \pm 52$ & $4895 \pm 47$ & $4782 \pm 44$ \\
$\bar{c}[c s][s q]$ & $4603 \pm 51$ & $4699 \pm 49$ & $4752 \pm 54$ & $4885 \pm 49$ & $4776 \pm 47$ \\
$\bar{c}[c q][s s]$ & $4913 \pm 51$ & $4981 \pm 49$ & $5038 \pm 54$ & $5170 \pm 49$ & $5061 \pm 47$ \\
\hline
\end{tabular}

are also presumably subject to the selection rules following from heavy quark symmetry. Thus, the state $\mathcal{P}_{X_{4}}$ (identified with $P_{c}(4380)^{+}$in [41]) is unlikely to be produced in $\Lambda_{b}$ decays, as it has the "wrong" light-diquark spin number. On the other hand, there is a lower mass state $\mathcal{P}_{X_{2}}$ present in the spectrum, having the correct flavor and spin quantum numbers $\left|1_{Q}, 0_{Q^{\prime}}, 0 ; \frac{3}{2}^{-}\right\rangle$, with a mass of about $4130 \mathrm{MeV}$, which we expect to be produced in $\Lambda_{b}$ decays. One could argue that the mass estimates following from the assumed effective Hamiltonian are in error by a larger amount than quoted in [339]. However, as already stated, the mass difference between the $J^{P}=\frac{5}{2}^{+}$and $J^{P}=\frac{3}{2}^{-}$pentaquarks, having the right quantum numbers $\left|1_{\mathcal{Q}}, 0_{\mathcal{Q}^{\prime}}, 1 ; \frac{5}{2}^{+}\right\rangle$and $\left|1_{\mathcal{Q}}, 0_{\mathcal{Q}^{\prime}}, 0 ; \frac{3}{2}^{-}\right\rangle$is expected to be around $340 \mathrm{MeV}$, yielding a mass for the lower-mass $J^{P}=\frac{3}{2}^{-}$pentaquark state of about $4110 \mathrm{MeV}$. The two estimates are compatible with each other, and we advocate to search for this state in the LHCb data. Among the ten states listed in Table 22, only the ones called $\mathcal{P}_{X_{2}}$ and $\mathcal{P}_{Y_{2}}$ are allowed as the $\Lambda_{b}$ decay products.

\subsection{Weak decays with $\mathbb{P}$ in decuplet representation}

Decays involving the decuplet 10 pentaquarks may also occur, if the light diquark pair having spin-0 $[u d]_{s=0}$ in $\Lambda_{b}$ gets broken to produce a spin-1 light diquark $[u d]_{s=1}$. In this case, one would also observe the decays of $\Lambda_{b}$, such as

$$
\begin{gathered}
\Lambda_{b} \rightarrow \pi \mathbb{P}_{10}^{(S=-1)} \rightarrow \pi(J / \psi \Sigma(1385)), \\
\Lambda_{b} \rightarrow K^{+} \mathbb{P}_{10}^{(S=-2)} \rightarrow K^{+}\left(J / \psi \Xi^{-}(1530)\right) .
\end{gathered}
$$

These decays are, however, disfavored by the heavy-quark-spin-conservation selection rules. The extent to which this rule is compatible with the existing data on $B$-meson and $\Lambda_{b}$ decays can be seen in the PDG entries. Whether the decays of the pentaquarks are also subject to the same selection rules is yet to be checked, but on symmetry grounds, we do expect it to hold. Hence, the observation (or not) of these decays will be quite instructive.

Apart from the $\Lambda_{b}(b u d)$, several other $b$-baryons, such as $\Xi_{b}^{0}(u s b), \Xi_{b}^{-}(d s b)$ and $\Omega_{b}^{-}(s s b)$ undergo weak decays. These $b$-baryons are characterized by the spin of the light diquark, as shown below, making their isospin $(I)$ and strangeness $(S)$ quantum numbers explicit as well as their light diquark 
$J^{\mathrm{P}}$ quantum numbers. The $c$-baryons are likewise characterized similarly. Examples of bottom-strange b-baryon in various charge combinations, respecting $\Delta I=0, \Delta S=-1$ are:

$$
\Xi_{b}^{0}(5794) \rightarrow K(J / \psi \Sigma(1385)),
$$

which corresponds to the formation of the pentaquarks with the spin configuration $\mathbb{P}_{10}\left(\bar{c}[c q]_{s=0,1}\left[q^{\prime} s\right]_{s=0,1}\right)$ with $\left(q, q^{\prime}=u, d\right)$.

The above stated considerations have been extended involving the entire $S U(3)_{F}$ multiplets entering the generic decay amplitude $\left\langle\mathcal{P} \mathcal{M}\left|H_{\text {eff }}\right| \mathcal{B}\right\rangle$, where $\mathcal{B}$ is the $S U(3)_{F}$ antitriplet $b$-baryon, shown in the left frame of Fig. $55, \mathcal{M}$ is the $3 \times 3$ pseudoscalar meson matrix

$$
\mathcal{M}_{i}^{j}=\left(\begin{array}{ccc}
\frac{\pi^{0}}{\sqrt{2}}+\frac{\eta_{8}}{\sqrt{6}} & \pi^{+} & K^{+} \\
\pi^{-} & -\frac{\pi^{0}}{\sqrt{2}}+\frac{\eta_{8}}{\sqrt{6}} & K^{0} \\
K^{-} & \bar{K}^{0} & -\frac{2 \eta_{8}}{\sqrt{6}}
\end{array}\right),
$$

and $\mathcal{P}$ is a pentaquark state belonging to an octet with definite $J^{P}$, denoted as a $3 \times 3$ matrix $J^{P}, \mathcal{P}_{j}^{i}\left(J^{\mathrm{P}}\right)$,

$$
\mathcal{P}_{i}^{j}\left(J^{P}\right)=\left(\begin{array}{ccc}
\frac{P_{\Sigma^{0}}}{\sqrt{2}}+\frac{P_{\Lambda}}{\sqrt{6}} & P_{\Sigma^{+}} & P_{p} \\
P_{\Sigma^{-}} & -\frac{P_{\Sigma^{0}}}{\sqrt{2}}+\frac{P_{\Lambda}}{\sqrt{6}} & P_{n} \\
P_{\Xi^{-}} & P_{\Xi^{0}} & -\frac{P_{\Lambda}}{\sqrt{6}}
\end{array}\right),
$$

or a decuplet $\mathcal{P}_{i j k}$ (symmetric in the indices), with $\mathcal{P}_{111}=\Delta_{10}^{++}, \ldots, \mathcal{P}_{333}=\Omega_{10}^{-}$. (see Guan-Nan Li et al. [333] for a detailed list of the component fields and $S U(3)_{F}$-based relations among decay widths). The two observed pentaquarks are denoted as $P_{p}\left(3 / 2^{-}\right)$and $P_{p}\left(5 / 2^{+}\right)$.

Estimates of the $S U(3)$ amplitudes require a dynamical model, which will be lot more complex to develop than the factorization-based models for the two-body $B$-meson decays, but, as argued in the literature, SU(3) symmetry can be used to relate different decay modes. Using heavy quark symmetry, which reduces the number of Feynman diagrams to be calculated, they are worked out in [339]. Thus, the decay $\Lambda_{b}^{0} \rightarrow J / \psi p K^{-}$and $\Lambda_{b}^{0} \rightarrow J / \psi p \pi^{-}$have just one dominant Feynman diagram each, the one in which the $[u d]$ diquark in $\Lambda_{b}^{0}$ retains its spin. The ratio of the branching fraction $\mathcal{B}\left(\Lambda_{b}^{0} \rightarrow\right.$ $\left.J / \psi p \pi^{-}\right) / \mathcal{B}\left(\Lambda_{b}^{0} \rightarrow J / \psi p K^{-}\right)=0.0824 \pm 0.0024 \pm 0.0042$ [106] is consistent with the expectations from Cabibbo suppression. This ratio should also hold for the resonating part of the amplitudes, namely if one replaces the $J / \psi p$ by $P_{c}(4450)^{+}$, and likewise for the $J^{P}=3 / 2^{-} P_{c}^{+}$state. This is hinted by the current LHCb measurements [342].

Examples of the weak decays in which the initial $b$-baryon has a spin-1 light diquark, i.e. $J^{\mathrm{P}}=1^{+}$, which is retained in the transition, are provided by the $\Omega_{b}$ decays. The $s \bar{s}$ pair in $\Omega_{b}$ is in the symmetric

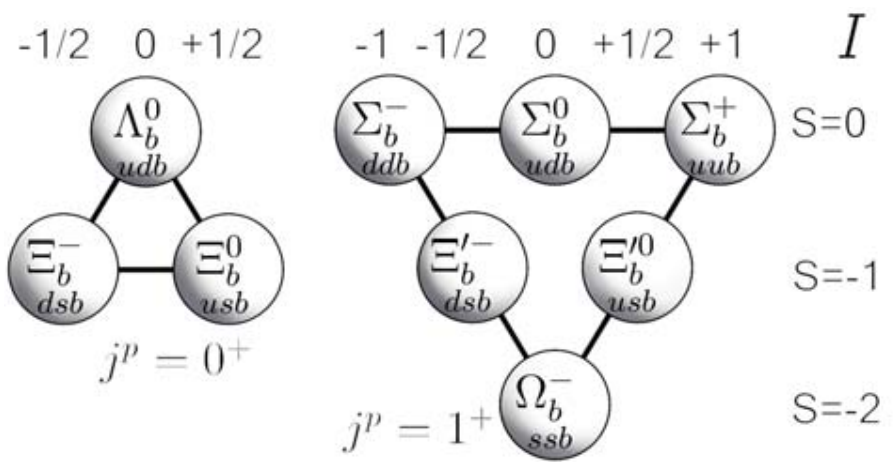

Figure 55: $b$-baryons with the light diquark spins $J^{p}=0^{+}$(left) and $J^{p}=1^{+}$(right). 
Table 24: Estimate of the ratios of the decay widths for $b$-baryon decays into hidden-charm pentaquarks $\Gamma\left(\mathcal{B}(\mathcal{C}) \rightarrow \mathcal{P}^{5 / 2} \mathcal{M}\right) / \Gamma\left(\Lambda_{b}^{0} \rightarrow P_{p}^{5 / 2} K^{-}\right)$for $\Delta S=1$ transitions (upper part of the table) and the Cabibbosuppressed $\Delta S=0$ transitions (lower part of the table) (from [339]).

\begin{tabular}{|c|c|c|c|}
\hline \multicolumn{4}{|l|}{$\Delta S=1$} \\
\hline Decay Process & $\Gamma / \Gamma\left(\Lambda_{b}^{0} \rightarrow P_{p}^{5 / 2} K^{-}\right)$ & Decay Process & $\Gamma / \Gamma\left(\Lambda_{b}^{0} \rightarrow P_{p}^{5 / 2} K^{-}\right)$ \\
\hline$\Lambda_{b} \rightarrow P_{p}^{\left\{Y_{2}\right\}_{c_{1}}} K^{-}$ & 1 & $\Xi_{b}^{-} \rightarrow P_{\Sigma^{-}}^{\left\{Y_{2}\right\}_{c_{2}}} \bar{K}^{0}$ & 2.07 \\
\hline$\Lambda_{b} \rightarrow P_{n}^{\left\{\left(Y_{2}\right\}_{c_{1}}\right.} \bar{K}^{0}$ & 1 & $\Xi_{b}^{0} \rightarrow P_{\Sigma^{+}}^{\left\{Y_{2}\right\}_{c_{2}}} K^{-}$ & 2.07 \\
\hline$\Lambda_{b} \rightarrow P_{\Lambda^{0}}^{\left\{Y_{2}\right\}_{c_{3}}} \eta^{\prime}$ & 0.03 & $\Lambda_{b} \rightarrow P_{\Lambda^{0}}^{\left\{Y_{2}\right\}_{c_{3}}} \eta$ & 0.19 \\
\hline$\Xi_{b}^{-} \rightarrow P_{\Sigma^{0}}^{\left\{Y_{2}\right\}_{c_{2}}} K^{-}$ & 1.04 & $\Xi_{b}^{-} \rightarrow P_{\Lambda^{0}}^{\left\{Y_{2}\right\}_{c_{2}}} K^{-}$ & 0.34 \\
\hline$\Omega_{b}^{-} \rightarrow P_{\Xi_{10}^{-}}^{\left\{Y_{3}\right\}_{c_{5}}} \bar{K}^{0}$ & 0.14 & $\Omega_{b}^{-} \rightarrow P_{\Xi_{10}^{0}}^{\left\{Y_{3}\right\}_{c_{5}}} K^{-}$ & 0.14 \\
\hline \multicolumn{4}{|l|}{$\Delta S=0$} \\
\hline Decay Process & $\Gamma / \Gamma\left(\Lambda_{b}^{0} \rightarrow P_{p}^{5 / 2} K^{-}\right)$ & Decay Process & $\Gamma / \Gamma\left(\Lambda_{b}^{0} \rightarrow P_{p}^{5 / 2} K^{-}\right)$ \\
\hline$\Lambda_{b} \rightarrow P_{p}^{\left\{Y_{2}\right\}_{c_{1}}} \pi^{-}$ & 0.08 & $\Lambda_{b} \rightarrow P_{n}^{\left\{Y_{2}\right\}_{c_{1}}} \pi^{0}$ & 0.04 \\
\hline$\Lambda_{b} \rightarrow P_{n}^{\left\{Y_{2}\right\}_{c_{1}}} \eta$ & 0.01 & $\Lambda_{b} \rightarrow P_{n}^{\left\{Y_{2}\right\}_{c_{1}}} \eta^{\prime}$ & 0 \\
\hline$\Xi_{b}^{-} \rightarrow P_{\Xi^{-}}^{\left\{Y_{2}\right\}_{c_{4}}} K^{0}$ & 0.02 & $\Xi_{b}^{-} \rightarrow P_{\Sigma^{0}}^{\left\{Y_{2}\right\}_{c_{2}}} \pi^{-}$ & 0.08 \\
\hline$\Xi_{b}^{-} \rightarrow P_{\Sigma^{-}}^{\left\{Y_{2}\right\}_{c_{2}}} \eta$ & 0.02 & $\Xi_{b}^{-} \rightarrow P_{\Sigma^{-}}^{\left\{Y_{2}\right\}_{c_{2}}} \eta^{\prime}$ & 0.01 \\
\hline$\Xi_{b}^{-} \rightarrow P_{\Sigma^{-}}^{\left\{Y_{2}\right\}_{c_{2}}} \pi^{0}$ & 0.08 & $\Xi_{b}^{0} \rightarrow P_{\Sigma^{0}}^{\left\{Y_{2}\right\}_{c_{2}}} \pi^{0}$ & 0.04 \\
\hline$\Xi_{b}^{0} \rightarrow P_{\Lambda^{0}}^{\left\{X_{2}\left(Y_{2}\right)\right\}_{c_{2}}} \eta$ & 0.01 & $\Xi_{b}^{0} \rightarrow P_{\Lambda^{0}}^{\left\{Y_{2}\right\}_{c_{2}}} \eta^{\prime}$ & 0.01 \\
\hline$\Xi_{b}^{0} \rightarrow P_{\Lambda^{0}}^{\left\{Y_{2}\right\}_{c_{2}}} \pi^{0}$ & 0.01 & $\Omega_{b}^{-} \rightarrow P_{\Xi_{10}^{-}}^{\left\{Y_{3}\right\}_{c_{5}}} \pi^{0}$ & 0.01 \\
\hline$\Omega_{b}^{-} \rightarrow P_{\Xi_{10}^{0}}^{\left\{Y_{3}\right\}_{c_{5}}} \pi^{-}$ & 0.02 & & \\
\hline
\end{tabular}

6 representation of $S U(3)_{F}$ with spin 1 and is expected to produce decuplet pentaquarks in association with a $\phi$ or a kaon 41]

$$
\Omega_{b}(6049) \rightarrow \phi\left(J / \psi \Omega^{-}(1672)\right), K(J / \psi \Xi(1387))
$$

These correspond, respectively, to the formation of the following pentaquarks $(q=u, d)$ :

$$
\mathbb{P}_{10}^{-}\left(\bar{c}[c s]_{s=0,1}[s s]_{s=1}\right), \mathbb{P}_{10}\left(\bar{c}[c q]_{s=0,1}[s s]_{s=1}\right)
$$

These transitions are expected on firmer theoretical footings, as the initial [ss] diquark in $\Omega_{b}$ is left unbroken. Again, a lot more transitions can be found relaxing this condition, which would involve a $J^{\mathrm{P}}=1^{+} \rightarrow 0^{+}$light diquark, but they are anticipated to be suppressed.

The ratios of $\Gamma\left(\mathcal{B}(\mathcal{C}) \rightarrow \mathcal{P}^{5 / 2} \mathcal{M}\right) / \Gamma\left(\Lambda_{b}^{0} \rightarrow P_{p}^{5 / 2} K^{-}\right)$for $\Delta S=1$ and the Cabibbo-suppressed $\Delta S=0$ transitions are given in Table 24. The suppression factor is $\left(V_{c d} / V_{c s}\right)^{2}$. Note that the pentaquark state $P_{p}^{5 / 2}$ denotes the state $P_{c}(4450)^{+}$with $J^{P}=5 / 2^{+}$. The corresponding ratios involving the $J^{P}=3 / 2^{-}$ pentaquark states are given in Table 25. 
Table 25: Estimate of the ratios of the decay widths $\Gamma\left(\mathcal{B}(\mathcal{C}) \rightarrow \mathcal{P}^{3 / 2} \mathcal{M}\right) / \Gamma\left(\Lambda_{b}^{0} \rightarrow P_{p}^{\left\{X_{2}\right\}_{c_{1}}} K^{-}\right)$for $\Delta S=1$ transitions (upper part of the table) and the Cabibbo-suppressed $\Delta S=0$ transitions (lower part of the table) (from 339$]$ ).

\begin{tabular}{|c|c|c|c|}
\hline \multicolumn{4}{|l|}{$\Delta S=1$} \\
\hline Decay Process & $\Gamma / \Gamma\left(\Lambda_{b}^{0} \rightarrow P_{p}^{\left\{X_{2}\right\}_{c_{1}} K^{-}}\right)$ & Decay Process & $\Gamma / \Gamma\left(\Lambda_{b}^{0} \rightarrow P_{p}^{\left\{X_{2}\right\}_{c_{1}}} K^{-}\right)$ \\
\hline$\Lambda_{b} \rightarrow P_{p}^{\left\{X_{2}\right\}_{c_{1}}} K^{-}$ & 1 & $\Xi_{b}^{-} \rightarrow P_{\Sigma^{-}}^{\left\{X_{2}\right\}_{c_{2}}} \bar{K}^{0}$ & 1.38 \\
\hline$\Lambda_{b} \rightarrow P_{n}^{\left\{X_{2}\right\}_{c_{1}}} \bar{K}^{0}$ & 1 & $\Xi_{b}^{0} \rightarrow P_{\Sigma^{+}}^{\left\{X_{2}\right\}_{c_{2}}} K^{-}$ & 1.38 \\
\hline$\Lambda_{b} \rightarrow P_{\Lambda^{0}}^{\left\{X_{2}\right\}_{c_{3}}} \eta^{\prime}$ & 0.17 & $\Lambda_{b} \rightarrow P_{\Lambda^{0}}^{\left\{X_{2}\right\}_{c_{3}}} \eta$ & 0.22 \\
\hline$\Xi_{b}^{-} \rightarrow P_{\Sigma^{0}}^{\left\{X_{2}\right\}_{c_{2}}} K^{-}$ & 0.69 & $\Xi_{b}^{-} \rightarrow P_{\Lambda^{0}}^{\left\{X_{2}\right\}_{c_{2}}} K^{-}$ & 0.23 \\
\hline$\Omega_{b}^{-} \rightarrow P_{\Xi_{10}^{-}}^{\left\{X_{3}\right\}_{c_{5}}} \bar{K}^{0}$ & 0.24 & $\Omega_{b}^{-} \rightarrow P_{\Xi_{10}^{0}}^{\left\{X_{3}\right\}_{c_{5}}} K^{-}$ & 0.24 \\
\hline \multicolumn{4}{|l|}{$\Delta S=0$} \\
\hline Decay Process & $\Gamma / \Gamma\left(\Lambda_{b}^{0} \rightarrow P_{p}^{\left\{X_{2}\right\}_{c_{1}} K^{-}}\right)$ & Decay Process & $\Gamma / \Gamma\left(\Lambda_{b}^{0} \rightarrow P_{p}^{\left\{X_{2}\right\}_{c_{1}}} K^{-}\right)$ \\
\hline$\Lambda_{b} \rightarrow P_{p}^{\left\{X_{2}\right\}_{c_{1}}} \pi^{-}$ & 0.06 & $\Lambda_{b} \rightarrow P_{n}^{\left\{X_{2}\right\}_{c_{1}}} \pi^{0}$ & 0.03 \\
\hline$\Lambda_{b} \rightarrow P_{n}^{\left\{X_{2}\right\}_{c_{1}}} \eta$ & 0.01 & $\Lambda_{b} \rightarrow P_{n}^{\left\{X_{2}\right\}_{c_{1}}} \eta^{\prime}$ & 0.01 \\
\hline$\Xi_{b}^{-} \rightarrow P_{\Xi^{-}}^{\left\{X_{2}\right\}_{c_{4}}} K^{0}$ & 0.02 & $\Xi_{b}^{-} \rightarrow P_{\Sigma^{0}}^{\left\{X_{2}\right\}_{c_{2}}} \pi^{-}$ & 0.03 \\
\hline$\Xi_{b}^{-} \rightarrow P_{\Sigma^{-}}^{\left\{X_{2}\right\}_{c_{2}}} \eta$ & 0.02 & $\Xi_{b}^{-} \rightarrow P_{\Sigma^{-}}^{\left\{X_{2}\right\}_{c_{2}}} \eta^{\prime}$ & 0.01 \\
\hline$\Xi_{b}^{-} \rightarrow P_{\Sigma^{-}}^{\left\{X_{2}\right\}_{c_{2}}} \pi^{0}$ & 0.04 & $\Xi_{b}^{0} \rightarrow P_{\Sigma^{0}}^{\left\{X_{2}\right\}_{c_{2}}} \pi^{0}$ & 0.02 \\
\hline$\Xi_{b}^{0} \rightarrow P_{\Lambda^{0}}^{\left\{X_{2}\right\}_{c_{2}}} \eta$ & 0 & $\Xi_{b}^{0} \rightarrow P_{\Lambda^{0}}^{\left\{X_{2}\right\}_{c_{2}}} \eta^{\prime}$ & 0 \\
\hline$\Xi_{b}^{0} \rightarrow P_{\Lambda^{0}}^{\left\{X_{2}\right\}_{c_{2}}} \pi^{0}$ & 0.01 & $\Omega_{b}^{-} \rightarrow P_{\Xi_{10}^{-}}^{\left\{X_{3}\right\}_{c_{5}}} \pi^{0}$ & 0.01 \\
\hline$\Omega_{b}^{-} \rightarrow P_{\Xi_{10}^{0}}^{\left\{X_{3}\right\}_{c_{5}}} \pi^{-}$ & 0.02 & & \\
\hline
\end{tabular}

\section{Summary}

In summary, with the discoveries of the $X, Y, Z$ and $P_{c}$ states a new era of hadron spectroscopy is upon us. In addition to the well-known $q \bar{q}$ mesons and $q q q$ baryons, there is increasing evidence that the hadronic world is multi-layered, in the form of tetraquark mesons, pentaquark baryons, and likely also the hexaquarks (or $H$ dibaryons) [343]. However, the underlying dynamics is far from being understood, and the real issue is how the various constituents of an exotic multiquark state rearrange themselves. The two competing pictures are hadron molecules and compact diquak models, with $Q \bar{Q} g$ hybrids and glueballs also anticipated. Thresholds near the resonances do play a role in the phenomenology, and in some cases kinematic-induced cusp effects may also be a viable template. It is plausible, perhaps rather likely, that no single mechanism fits all the observable states, and the exotic hadrons may find their abode in competing theoretical frameworks. The case of diquark models in this context was reviewed here in more detail. Existence proof on the lattice of diquark correlations in some of the tetra- and pentaquark states discussed here would be a breakthrough and keenly awaited. In the meanwhile, phenomenological models built within constrained theoretical frameworks are unavoidable. They and experiments will guide us how to navigate through this uncharted territory.

We thank Luciano Maiani, Christoph Hanhart, Antonello Polosa and Gerrit Schierholz for helpful 
discussions. Our colleagues in the Belle and LHCb experiments provided a great deal of input and useful discussions. S. Stone thanks the U. S. National Science Foundation for support. 


\section{References}

[1] A. J. Bevan, et al., The physics of the $B$ factories, Eur. Phys. J. C74 (2014) 3026 . arXiv: 1406.6311, doi:10.1140/epjc/s10052-014-3026-9.

[2] S. Choi, et al., Observation of a resonance-like structure in the $\pi^{ \pm} \psi^{\prime}$ mass distribution in exclusive $B \rightarrow K \pi^{ \pm} \psi^{\prime}$ decays, Phys.Rev.Lett. 100 (2008) 142001. arXiv:0708.1790, doi: 10.1103/PhysRevLett.100.142001.

[3] B. Aubert, et al., Study of the $B \rightarrow J / \psi K^{-} \pi^{+} \pi^{-}$decay and measurement of the $B \rightarrow X(3872) K^{-}$ branching fraction, Phys. Rev. D71 (2005) 071103. arXiv:hep-ex/0406022, doi:10.1103/ PhysRevD .71.071103.

[4] B. Aubert, et al., A study of $B \rightarrow X(3872) K$, with $X(3872) \rightarrow J / \Psi \pi^{+} \pi^{-}$, Phys. Rev. D77 (2008) 111101. arXiv:0803.2838, doi:10.1103/PhysRevD.77.111101.

[5] D. Acosta, et al., Observation of the narrow state $X(3872) \rightarrow J / \psi \pi^{+} \pi^{-}$in $\bar{p} p$ collisions at $\sqrt{s}=1.96 \mathrm{TeV}$, Phys. Rev. Lett. 93 (2004) 072001. arXiv:hep-ex/0312021, doi:10.1103/ PhysRevLett.93.072001.

[6] A. Abulencia, et al., Measurement of the dipion mass spectrum in $X(3872) \rightarrow J / \psi \pi^{+} \pi^{-}$decays., Phys. Rev. Lett. 96 (2006) 102002. arXiv:hep-ex/0512074, doi:10.1103/PhysRevLett.96. 102002 .

[7] T. Aaltonen, et al., Precision Measurement of the $X(3872)$ Mass in $J / \psi \pi^{+} \pi^{-}$Decays, Phys. Rev. Lett. 103 (2009) 152001. arXiv:0906.5218, doi:10.1103/PhysRevLett.103.152001.

[8] V. M. Abazov, et al., Observation and properties of the $X(3872)$ decaying to $J / \psi \pi^{+} \pi^{-}$in $p \bar{p}$ collisions at $\sqrt{s}=1.96 \mathrm{TeV}$, Phys. Rev. Lett. 93 (2004) 162002. arXiv:hep-ex/0405004, doi: 10.1103/PhysRevLett.93.162002.

[9] R. Aaij, et al., Observation of $X(3872)$ production in $p p$ collisions at $\sqrt{s}=7$ TeV, Eur. Phys. J. C72 (2012) 1972. arXiv:1112.5310, doi:10.1140/epjc/s10052-012-1972-7.

[10] S. Chatrchyan, et al., Measurement of the $X(3872)$ production cross section via decays to $J / \psi$ pi pi in pp collisions at sqrt(s) $=7 \mathrm{TeV}$, JHEP 04 (2013) 154. arXiv:1302.3968, doi:10.1007/ JHEP04(2013) 154.

[11] R. Aaij, et al., Observation of $J / \psi p$ resonances consistent with pentaquark states in $\Lambda_{b}^{0} \rightarrow J / \psi K^{-} p$ decays, Phys. Rev. Lett. 115 (2015) 072001. arXiv:1507.03414, doi:10.1103/PhysRevLett. 115.072001 .

[12] K. Olive, et al., Review of particle physics, Chin.Phys. C38 (2014) 090001. doi:10.1088/ 1674-1137/38/9/090001.

[13] R. F. Lebed, R. E. Mitchell, E. S. Swanson, Heavy-quark QCD exotica, Prog. Part. Nucl. Phys. 93 (2017) 143-194. arXiv:1610.04528, doi:10.1016/j.ppnp.2016.11.003.

[14] A. Esposito, A. Pilloni, A. D. Polosa, Multiquark resonances, Phys. Rept. 668 (2016) 1-97. arXiv: 1611.07920, doi:10.1016/j.physrep.2016.11.002.

[15] F.-K. Guo, C. Hanhart, U.-G. Meißner, Q. Wang, Q. Zhao, B.-S. Zou, Hadronic molecules, arXiv: 1705.00141 . 
[16] I. Adachi, et al., First observation of the $P$-wave spin-singlet bottomonium states $h_{b}(1 P)$ and $h_{b}(2 P)$, Phys. Rev. Lett. 108 (2012) 032001. arXiv:1103.3419, doi:10.1103/PhysRevLett. 108.032001 .

[17] E. Eichten, K. Gottfried, T. Kinoshita, K. D. Lane, T.-M. Yan, Charmonium: The Model, Phys. Rev. D17 (1978) 3090, [Erratum: Phys. Rev.D21,313(1980)]. doi:10.1103/PhysRevD.17.3090, 10.1103/PhysRevD.21.313.

[18] E. Eichten, K. Gottfried, T. Kinoshita, K. D. Lane, T.-M. Yan, Charmonium: comparison with experiment, Phys. Rev. D21 (1980) 203. doi:10.1103/PhysRevD.21.203.

[19] N. Brambilla, et al., Heavy quarkonium physics, arXiv:hep-ph/0412158.

[20] N. Brambilla, et al., Heavy quarkonium: progress, puzzles, and opportunities, Eur. Phys. J. C71 (2011) 1534. arXiv:1010.5827, doi:10.1140/epjc/s10052-010-1534-9.

[21] A. Gray, I. Allison, C. T. H. Davies, E. Dalgic, G. P. Lepage, J. Shigemitsu, M. Wingate, The Upsilon spectrum and $\mathrm{m}(\mathrm{b})$ from full lattice QCD, Phys. Rev. D72 (2005) 094507. arXiv: hep-lat/0507013, doi:10.1103/PhysRevD.72.094507.

[22] R. J. Dowdall, et al., The Upsilon spectrum and the determination of the lattice spacing from lattice QCD including charm quarks in the sea, Phys. Rev. D85 (2012) 054509. arXiv:1110.6887, doi:10.1103/PhysRevD.85.054509.

[23] G. S. Bali, S. Collins, C. Ehmann, Charmonium spectroscopy and mixing with light quark and open charm states from $n_{F}=2$ lattice QCD, Phys. Rev. D84 (2011) 094506. arXiv:1110.2381, doi:10.1103/PhysRevD.84.094506.

[24] L. Liu, G. Moir, M. Peardon, S. M. Ryan, C. E. Thomas, P. Vilaseca, J. J. Dudek, R. G. Edwards, B. Joo, D. G. Richards, Excited and exotic charmonium spectroscopy from lattice QCD, JHEP 07 (2012) 126. arXiv:1204.5425, doi:10.1007/JHEP07(2012) 126.

[25] S. Prelovsek, Hadron spectroscopy, PoS LATTICE2014 (2014) 015. arXiv:1411.0405.

[26] T. Barnes, S. Godfrey, E. S. Swanson, Higher charmonia, Phys. Rev. D72 (2005) 054026. arXiv: hep-ph/0505002, doi:10.1103/PhysRevD.72.054026.

[27] A. Bondar, et al., Observation of two charged bottomonium-like resonances in $\mathrm{Y}(5 \mathrm{~S})$ decays, Phys.Rev.Lett. 108 (2012) 122001. arXiv:1110.2251, doi:10.1103/PhysRevLett.108.122001.

[28] L. S. Brown, R. N. Cahn, Chiral symmetry and $\psi^{\prime} \rightarrow \psi \pi \pi$ decay, Phys. Rev. Lett. 35 (1975) 1. doi:10.1103/PhysRevLett.35.1.

[29] M. B. Voloshin, Adler's self consistency condition in the decay $\psi^{\prime}(3700) \rightarrow J / \psi(3100) \pi \pi$, JETP Lett. 21 (1975) 347-348, [Pisma Zh. Eksp. Teor. Fiz.21,733(1975)].

[30] A. Abdesselam, et al., Energy scan of the $e^{+} e^{-} \rightarrow h_{b}(n P) \pi^{+} \pi^{-}(n=1,2)$ cross sections and evidence for $\Upsilon(11020)$ decays into charged bottomonium-like states, Phys. Rev. Lett. 117 (14) (2016) 142001. arXiv:1508.06562, doi:10.1103/PhysRevLett.117.142001.

[31] N. A. Törnqvist, Understanding the scalar meson $q \bar{q}$ nonet, Z. Phys. C68 (1995) 647-660. arXiv: hep-ph/9504372, doi:10.1007/BF01565264.

[32] D. Bugg, An explanation of Belle states $Z_{b}(10610)$ and $Z_{b}(10650)$, Europhys. Lett. 96 (2011) 11002. arXiv:1105.5492, doi:10.1209/0295-5075/96/11002. 
[33] J. Vijande, E. Weissman, A. Valcarce, N. Barnea, Are there compact heavy four-quark bound states?, Phys. Rev. D76 (2007) 094027. arXiv:0710.2516, doi:10.1103/PhysRevD.76.094027.

[34] R. L. Jaffe, Multiquark hadrons. I. Phenomenology of $Q^{2} \bar{Q}^{2}$ mesons, Phys.Rev. D15 (1977) 267. doi:10.1103/PhysRevD.15.267.

[35] R. L. Jaffe, F. Wilczek, Diquarks and exotic spectroscopy, Phys.Rev.Lett. 91 (2003) 232003. arXiv:hep-ph/0307341, doi:10.1103/PhysRevLett.91.232003.

[36] F. Wilczek, Diquarks as inspiration and as objects, in: From fields to strings: Circumnavigating theoretical physics. Ian Kogan memorial collection (3 volume set), 2004, pp. 322-338, [,77(2004)]. arXiv:hep-ph/0409168, doi:10.1142/9789812775344_0007.

[37] M. G. Alford, K. Rajagopal, F. Wilczek, QCD at finite baryon density: Nucleon droplets and color superconductivity, Phys. Lett. B422 (1998) 247-256. arXiv:hep-ph/9711395, doi:10. 1016/S0370-2693(98)00051-3.

[38] L. Maiani, F. Piccinini, A. D. Polosa, V. Riquer, A new look at scalar mesons, Phys. Rev. Lett. 93 (2004) 212002. arXiv:hep-ph/0407017, doi:10.1103/PhysRevLett.93.212002.

[39] L. Maiani, F. Piccinini, A. D. Polosa, V. Riquer, Diquark-antidiquarks with hidden or open charm and the nature of X(3872), Phys. Rev. D71 (2005) 014028. arXiv:hep-ph/0412098, doi: 10.1103/PhysRevD.71.014028.

[40] S. J. Brodsky, D. S. Hwang, R. F. Lebed, Dynamical picture for the formation and decay of the exotic XYZ mesons, Phys. Rev. Lett. 113 (11) (2014) 112001. arXiv:1406.7281, doi: 10.1103/PhysRevLett.113.112001.

[41] L. Maiani, A. D. Polosa, V. Riquer, The new pentaquarks in the diquark model, Phys. Lett. B749 (2015) 289-291. arXiv:1507.04980, doi:10.1016/j.physletb.2015.08.008.

[42] M. Voloshin, L. Okun, Hadron molecules and charmonium atom, JETP Lett. 23 (1976) 333-336.

[43] S. Dubynskiy, M. B. Voloshin, Hadro-charmonium, Phys. Lett. B666 (2008) 344-346. arXiv: 0803.2224, doi:10.1016/j.physletb.2008.07.086.

[44] A. De Rujula, H. Georgi, S. Glashow, Molecular charmonium: a new spectroscopy?, Phys.Rev.Lett. 38 (1977) 317. doi:10.1103/PhysRevLett.38.317.

[45] N. A. Törnqvist, Possible large deuteron-like meson meson states bound by pions, Phys.Rev.Lett. 67 (1991) 556-559. doi:10.1103/PhysRevLett.67.556.

[46] N. A. Törnqvist, From the deuteron to deusons, an analysis of deuteron-like meson meson bound states, Z.Phys. C61 (1994) 525-537. arXiv:hep-ph/9310247, doi:10.1007/BF01413192.

[47] F. E. Close, P. R. Page, Gluonic charmonium resonances at BaBar and BELLE?, Phys. Lett. B628 (2005) 215-222. arXiv:hep-ph/0507199, doi:10.1016/j.physletb.2005.09.016.

[48] R. Aaij, et al., Observation of five new narrow $\Omega_{c}^{0}$ states decaying to $\Xi_{c}^{+} K^{-}, \operatorname{arXiv:1703.04639.}$

[49] W. Wang, R.-L. Zhu, Interpretation of the newly observed $\Omega_{c}^{0}$ resonance, arXiv:1704.00179.

[50] M. Padmanath, N. Mathur, Quantum numbers of recently discovered $\Omega_{c}^{0}$ baryons from lattice QCD, arXiv:1704.00259. 
[51] T. M. Aliev, S. Bilmis, M. Savci, Are the new excited $\Omega_{c}$ baryons negative parity states?, arXiv: 1704.03439 .

[52] M. Karliner, J. L. Rosner, Very narrow excited $\Omega_{c}$ baryons, arXiv:1703.07774.

[53] H.-X. Chen, W. Chen, X. Liu, S.-L. Zhu, The hidden-charm pentaquark and tetraquark states, Phys. Rept. 639 (2016) 1-121. arXiv:1601.02092, doi:10.1016/j.physrep.2016.05.004.

[54] S. Godfrey, N. Isgur, Mesons in a relativized quark model with chromodynamics, Phys. Rev. D32 (1985) 189-231. doi:10.1103/PhysRevD.32.189.

[55] E. J. Eichten, K. Lane, C. Quigg, Charmonium levels near threshold and the narrow state $X(3872) \rightarrow \pi^{+} \pi^{-} J / \psi$, Phys. Rev. D69 (2004) 094019. arXiv:hep-ph/0401210, doi:10.1103/ PhysRevD.69.094019.

[56] R. J. Dowdall, C. T. H. Davies, T. C. Hammant, R. R. Horgan, Precise heavy-light meson masses and hyperfine splittings from lattice QCD including charm quarks in the sea, Phys. Rev. D86 (2012) 094510. arXiv:1207.5149, doi:10.1103/PhysRevD.86.094510.

[57] J. O. Daldrop, C. T. H. Davies, R. J. Dowdall, Prediction of the bottomonium D-wave spectrum from full lattice QCD, Phys. Rev. Lett. 108 (2012) 102003. arXiv:1112.2590, doi:10.1103/ PhysRevLett.108.102003.

[58] G. C. Donald, C. T. H. Davies, R. J. Dowdall, E. Follana, K. Hornbostel, J. Koponen, G. P. Lepage, C. McNeile, Precision tests of the $J / \psi$ from full lattice QCD: mass, leptonic width and radiative decay rate to $\eta_{c}$, Phys. Rev. D86 (2012) 094501. arXiv:1208.2855, doi:10.1103/ PhysRevD.86.094501.

[59] G. K. C. Cheung, C. O’Hara, G. Moir, M. Peardon, S. M. Ryan, C. E. Thomas, D. Tims, Excited and exotic charmonium, $D_{s}$ and $D$ meson spectra for two light quark masses from lattice QCD, JHEP 12 (2016) 089. arXiv:1610.01073, doi:10.1007/JHEP12(2016)089.

[60] M. Lüscher, Two particle states on a torus and their relation to the scattering matrix, Nucl. Phys. B354 (1991) 531-578. doi:10.1016/0550-3213(91)90366-6.

[61] M. Lüscher, Signatures of unstable particles in finite volume, Nucl. Phys. B364 (1991) 237-251. doi : 10.1016/0550-3213(91)90584-K.

[62] J. M. M. Hall, A. C. P. Hsu, D. B. Leinweber, A. W. Thomas, R. D. Young, Finite-volume matrix Hamiltonian model for a $\Delta \rightarrow N \pi$ system, Phys. Rev. D87 (9) (2013) 094510. arXiv:1303.4157, doi:10.1103/PhysRevD.87.094510.

[63] N. Ishii, S. Aoki, T. Doi, T. Hatsuda, Y. Ikeda, T. Inoue, K. Murano, H. Nemura, K. Sasaki, Hadron-hadron interactions from imaginary-time Nambu-Bethe-Salpeter wave function on the lattice, Phys. Lett. B712 (2012) 437-441. arXiv:1203.3642, doi:10.1016/j.physletb.2012. 04.076.

[64] T. Yamazaki, Hadronic Interactions, PoS LATTICE2014 (2015) 009. arXiv:1503.08671.

[65] R. A. Briceo, Few-body physics, PoS LATTICE2014 (2015) 008. arXiv:1411.6944.

[66] S. R. Beane, E. Chang, S. D. Cohen, W. Detmold, H. W. Lin, K. Orginos, A. Parreo, M. J. Savage, Quarkonium-nucleus bound states from lattice QCD, Phys. Rev. D91 (11) (2015) 114503. arXiv:1410.7069, doi:10.1103/PhysRevD.91.114503. 
[67] S. Prelovsek, Hadron spectroscopy and interactions from lattice QCD, EPJ Web Conf. 129 (2016) 00018. arXiv:1609.03052, doi:10.1051/epjconf/201612900018.

[68] S. Prelovsek, L. Leskovec, Evidence for $X(3872)$ from $D D^{*}$ scattering on the lattice, Phys. Rev. Lett. 111 (2013) 192001. arXiv:1307.5172, doi:10.1103/PhysRevLett.111.192001.

[69] S.-h. Lee, C. DeTar, H. Na, D. Mohler, Searching for the $X(3872)$ and $Z_{c}^{+}(3900)$ on HISQ lattices, arXiv:1411.1389.

[70] M. Padmanath, C. B. Lang, S. Prelovsek, X(3872) and Y(4140) using diquark-antidiquark operators with lattice QCD, Phys. Rev. D92 (3) (2015) 034501. arXiv:1503.03257, doi: 10.1103/PhysRevD.92.034501.

[71] Y. Ikeda, S. Aoki, T. Doi, S. Gongyo, T. Hatsuda, T. Inoue, T. Iritani, N. Ishii, K. Murano, K. Sasaki, Fate of the tetraquark candidate $Z_{c}(3900)$ from lattice QCD, Phys. Rev. Lett. 117 (24) (2016) 242001. arXiv:1602.03465, doi:10.1103/PhysRevLett.117.242001.

[72] M. Born, R. Oppenheimer, Zur Quantentheorie der Molekln, Annalen der Physik 389 (1927) 457. doi:10.1002/andp.19273892002.

[73] K. J. Juge, J. Kuti, C. J. Morningstar, Ab initio study of hybrid $\bar{b} g b$ mesons, Phys. Rev. Lett. 82 (1999) 4400-4403. arXiv:hep-ph/9902336, doi:10.1103/PhysRevLett.82.4400.

[74] E. Braaten, C. Langmack, D. H. Smith, Born-Oppenheimer approximation for the $X Y Z$ mesons, Phys. Rev. D90 (1) (2014) 014044. arXiv:1402.0438, doi:10.1103/PhysRevD.90.014044.

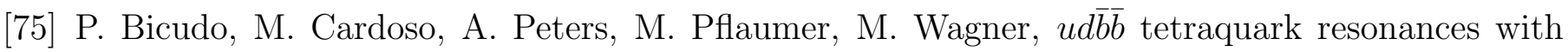
lattice QCD potentials and the Born-Oppenheimer approximation, arXiv:1704.02383.

[76] A. Francis, R. J. Hudspith, R. Lewis, K. Maltman, Lattice prediction for deeply bound doubly heavy tetraquarks, Phys. Rev. Lett. 118 (14) (2017) 142001. arXiv:1607.05214, doi:10.1103/ PhysRevLett.118.142001.

[77] P. Bicudo, K. Cichy, A. Peters, M. Wagner, BB interactions with static bottom quarks from Lattice QCD, Phys. Rev. D93 (3) (2016) 034501. arXiv:1510.03441, doi:10.1103/PhysRevD. 93.034501.

[78] D. Strottman, Multi-quark baryons and the MIT bag model, Phys.Rev. D20 (1979) 748-767. doi:10.1103/PhysRevD.20.748.

[79] H. J. Lipkin, New possibilities for exotic hadrons: Anticharmed strange baryons, Phys.Lett. B195 (1987) 484. doi:10.1016/0370-2693(87)90055-4.

[80] C. Gignoux, B. Silvestre-Brac, J. M. Richard, Possibility of stable multi-quark baryons, Phys. Lett. B193 (1987) 323. doi:10.1016/0370-2693(87)91244-5.

[81] K. H. Hicks, On the conundrum of the pentaquark, Eur.Phys.J. H37 (2012) 1-31. doi:10.1140/ epjh/e2012-20032-0.

[82] T. Nakano, et al., Evidence for a narrow $\mathrm{S}=+1$ baryon resonance in photoproduction from the neutron, Phys.Rev.Lett. 91 (2003) 012002. arXiv:hep-ex/0301020, doi:10.1103/PhysRevLett. 91.012002. 
[83] V. Barmin, et al., Observation of a baryon resonance with positive strangeness in $K^{+}$collisions with Xe nuclei, Phys.Atom.Nucl. 66 (2003) 1715-1718. arXiv:hep-ex/0304040, doi:10.1134/ 1.1611587 .

[84] S. Stepanyan, et al., Observation of an exotic $\mathrm{S}=+1$ baryon in exclusive photoproduction from the deuteron, Phys.Rev.Lett. 91 (2003) 252001. arXiv:hep-ex/0307018, doi:10.1103/ PhysRevLett.91.252001.

[85] J. Barth, et al., Evidence for the positive strangeness pentaquark $\Theta^{+}$in photoproduction with the SAPHIR detector at ELSA, Phys.Lett. B572 (2003) 127-132. arXiv:hep-ex/0307083, doi: $10.1016 / j \cdot$ physletb.2003.08.019.

[86] C. Alt, et al., Observation of an exotic $\mathrm{S}=-2, \mathrm{Q}=-2$ baryon resonance in proton proton collisions at the CERN SPS, Phys.Rev.Lett. 92 (2004) 042003. arXiv:hep-ex/0310014, doi: 10.1103/PhysRevLett.92.042003.

[87] I. Abt, et al., Limits for the central production of $\Theta^{+}$and $\Xi^{--}$pentaquarks in $920-\mathrm{GeV}$ pA collisions, Phys.Rev.Lett. 93 (2004) 212003. arXiv:hep-ex/0408048, doi:10.1103/PhysRevLett. 93.212003.

[88] A. Aktas, et al., Evidence for a narrow anti-charmed baryon state, Phys.Lett. B588 (2004) 17. arXiv:hep-ex/0403017, doi:10.1016/j.physletb.2004.03.012.

[89] S. Chekanov, et al., Search for a narrow charmed baryonic state decaying to $D^{*-} p$ in $e p$ collisions at HERA, Eur.Phys.J. C38 (2004) 29-41. arXiv:hep-ex/0409033, doi:10.1140/epjc/ s2004-02042-9.

[90] A. Dzierba, D. Krop, M. Swat, S. Teige, A. Szczepaniak, The evidence for a pentaquark signal and kinematic reflections, Phys.Rev. D69 (2004) 051901. arXiv:hep-ph/0311125, doi:10.1103/ PhysRevD.69.051901.

[91] S. Stone, Pathological science, in: Flavor physics for the millennium. Proceedings, Theoretical Advanced Study Institute in elementary particle physics, TASI 2000, Boulder, USA, June 4-30, 2000, 2000, pp. 557-575. arXiv:hep-ph/0010295.

URL http://alice.cern.ch/format/showfull?sysnb=2225959

[92] T. L. Collaboration, Letter of Intent for the LHCb Upgrade.

[93] R. Aaij, et al., Measurement of $b$-hadron production fractions in $7 \mathrm{TeV}$ pp collisions, Phys. Rev. D85 (2012) 032008. arXiv:1111.2357, doi:10.1103/PhysRevD.85.032008.

[94] R. Aaij, et al., Precision measurement of the ratio of the $\Lambda_{b}^{0}$ to $\bar{B}^{0}$ lifetimes, Phys.Lett. B734 (2014) 122-130. arXiv:1402.6242, doi:10.1016/j.physletb.2014.05.021.

[95] R. Dalitz, On the analysis of $\tau$-meson data and the nature of the $\tau$-meson, Phil.Mag. 44 (1953) 1068-1080. doi:10.1080/14786441008520365.

[96] J. F. Donoghue, E. Golowich, W. A. Ponce, B. R. Holstein, Analysis of $\Delta S=1$ nonleptonic weak decays and the $\Delta \mathrm{I}=1 / 2$ rule, Phys.Rev. D21 (1980) 186. doi:10.1103/PhysRevD.21.186.

[97] S. M. Flatté, Coupled-channel analysis of the $\pi \eta$ and $K \bar{K}$ systems near $K \bar{K}$ threshold, Phys.Lett. B63 (1976) 224. doi:10.1016/0370-2693(76) 90654-7.

[98] S. U. Chung, Spin formalisms, cERN-71-08 (1971). 
[99] J. D. Richman, An experimenter's guide to the helicity formalism, cALT-68-1148 (1984).

[100] M. Jacob, G. Wick, On the general theory of collisions for particles with spin, Annals Phys. 7 (1959) 404-428. doi:10.1016/0003-4916(59)90051-X.

[101] Soffer, Jacques and Törnqvist, Nils A., Origin of the polarization for inclusive $\Lambda$ production in $p p$ collisions, Phys. Rev. Lett. 68 (1992) 907-910. doi:10.1103/PhysRevLett.68.907.

[102] F. James, Statistical methods in experimental physics, World Scientific Publishing, 2006.

[103] R. Aaij, et al., Study of the production of $\Lambda_{b}^{0}$ and $\bar{B}^{0}$ hadrons in $p p$ collisions and first measurement of the $\Lambda_{b}^{0} \rightarrow J / \psi p K^{-}$branching fraction, Chin. Phys. C40 (1) (2016) 011001. arXiv:1509.00292, doi:10.1088/1674-1137/40/1/011001.

[104] S. Stone, Pentaquarks and tetraquarks at LHCb, PoS EPS-HEP2015 (2015) 434. arXiv:1509. 04051 .

[105] H.-Y. Cheng, C.-K. Chua, Bottom baryon decays to pseudoscalar meson and pentaquark, Phys. Rev. D92 (9) (2015) 096009. arXiv:1509.03708, doi:10.1103/PhysRevD.92.096009.

[106] R. Aaij, et al., Observation of the $\Lambda_{b}^{0} \rightarrow J / \psi p \pi^{-}$decay, JHEP 07 (2014) 103. arXiv:1406.0755, doi:10.1007/JHEP07(2014)103.

[107] T. Skwarnicki, A study of the radiative cascade transitions between the $\Upsilon$ and $\Upsilon^{\prime}$, Ph.D. thesis, Cracow, INP (1986).

URL http://lss.fnal.gov/cgi-bin/find_paper.pl?other/thesis/skwarnicki.pdf

[108] M. Pivk, F. R. Le Diberder, sPlot: A statistical tool to unfold data distributions, Nucl.Instrum.Meth. A555 (2005) 356-369. arXiv:physics/0402083, doi:10.1016/j.nima. 2005.08.106.

[109] M. Ablikim, et al., Observation of two new $N^{*}$ resonances in the decay $\psi(3686) \rightarrow p \bar{p} \pi^{0}$, Phys. Rev. Lett. 110 (2) (2013) 022001. arXiv:1207.0223, doi:10.1103/PhysRevLett.110.022001.

[110] B. Aubert, et al., Search for the Z(4430)- at BaBar, Phys. Rev. D79 (2009) 112001. arXiv: 0811.0564, doi:10.1103/PhysRevD.79.112001.

[111] R. Aaij, et al., Model-independent confirmation of the $Z(4430)^{-}$state, Phys. Rev. D92 (11) (2015) 112009, [Phys. Rev.D92,112009(2015)]. arXiv:1510.01951, doi:10.1103/PhysRevD.92.112009.

[112] U. Loring, B. C. Metsch, H. R. Petry, The light baryon spectrum in a relativistic quark model with instanton induced quark forces: the nonstrange baryon spectrum and ground states, Eur. Phys. J. A10 (2001) 395-446. arXiv:hep-ph/0103289, doi:10.1007/s100500170105.

[113] Q. Wang, X.-H. Liu, Q. Zhao, Photoproduction of hidden charm pentaquark states $P_{c}^{+}(4380)$ and $P_{c}^{+}$(4450), Phys. Rev. D92 (2015) 034022. arXiv:1508.00339, doi:10.1103/PhysRevD.92. 034022 .

[114] V. Kubarovsky, M. B. Voloshin, Formation of hidden-charm pentaquarks in photon-nucleon collisions, Phys. Rev. D92 (3) (2015) 031502. arXiv:1508.00888, doi:10.1103/PhysRevD.92. 031502 .

[115] M. Karliner, J. L. Rosner, Photoproduction of Exotic Baryon Resonances, Phys. Lett. B752 (2016) 329-332. arXiv:1508.01496, doi:10.1016/j.physletb.2015.11.068. 
[116] J. J. Sakurai, Theory of strong interactions, Annals Phys. 11 (1960) 1-48. doi:10.1016/ 0003-4916(60)90126-3.

[117] A. N. H. Blin, C. Fernndez-Ramrez, A. Jackura, V. Mathieu, V. I. Mokeev, A. Pilloni, A. P. Szczepaniak, Studying the $\mathrm{P}_{c}(4450)$ resonance in $\mathrm{J} / \psi$ photoproduction off protons, Phys. Rev. D94 (3) (2016) 034002. arXiv:1606.08912, doi:10.1103/PhysRevD.94.034002.

[118] V. Kubarovsky, M. B. Voloshin, Search for hidden-charm pentaquark with CLAS12, arXiv:1609. 00050 .

[119] Z. E. Meziani, et al., A search for the LHCb charmed 'pentaquark' using photo-production of $J / \psi$ at threshold in Hall C at Jefferson Lab, arXiv:1609.00676.

[120] L. Antoniazzi, et al., Search for hidden charm resonance states decaying into $J / \psi$ or $\psi^{\prime}$ plus pions, Phys. Rev. D50 (1994) 4258-4264. doi:10.1103/PhysRevD.50.4258.

[121] S. K. Choi, et al., Observation of a narrow charmonium-like state in exclusive $B^{+-} \rightarrow$ $K^{+-} \pi^{+} \pi^{-} J / \psi$ decays, Phys. Rev. Lett. 91 (2003) 262001. arXiv:hep-ex/0309032, doi: 10.1103/PhysRevLett.91.262001.

[122] S. K. Choi, et al., Bounds on the width, mass difference and other properties of $X(3872) \rightarrow$ $\pi^{+} \pi^{-} J / \psi$ decays, Phys. Rev. D84 (2011) 052004. arXiv:1107.0163, doi:10.1103/PhysRevD. 84.052004 .

[123] R. Aaij, et al., Determination of the $X(3872)$ meson quantum numbers, Phys. Rev. Lett. 110 (2013) 222001. arXiv:1302.6269, doi:10.1103/PhysRevLett.110.222001.

[124] E. Braaten, M. Lu, Line shapes of the X(3872), Phys. Rev. D76 (2007) 094028. arXiv:0709.2697, doi:10.1103/PhysRevD.76.094028.

[125] E. Braaten, M. Lu, The effects of charged charm mesons on the line shapes of the X(3872), Phys. Rev. D77 (2008) 014029. arXiv:0710.5482, doi:10.1103/PhysRevD.77.014029.

[126] E. Braaten, M. Kusunoki, Low-energy universality and the new charmonium resonance at 3870MeV, Phys. Rev. D69 (2004) 074005. arXiv:hep-ph/0311147, doi:10.1103/PhysRevD.69. 074005 .

[127] M. J. Galuska, W. Kuhn, J. S. Lange, B. Spruck, Results for a simulated resonance scan of the $X(3872)$ at PANDA, PoS BORMIO2012 (2012) 018.

[128] S. Lange, M. Galuska, S. Reiter, E. Prencipe, S. Spataro, New Studies of XYZ States at PANDA, in: Proceedings, 6th International Workshop on Charm Physics (Charm 2013): Manchester, UK, August 31-September 4, 2013, 2013. arXiv:1311.7597.

URL http://inspirehep.net/record/1266764/files/arXiv:1311.7597.pdf

[129] K. Abe, et al., Evidence for $X(3872) \rightarrow \gamma J / \psi$ and the sub-threshold decay $X(3872) \rightarrow \omega J / \psi$, in: Lepton and photon interactions at high energies. Proceedings, 22nd International Symposium, LP 2005, Uppsala, Sweden, June 30-July 5, 2005, 2005. arXiv:hep-ex/0505037.

[130] B. Aubert, et al., Search for $B^{+} \rightarrow X(3872) K^{+}, X(3872) \rightarrow J / \psi \gamma$, Phys. Rev. D74 (2006) 071101. arXiv:hep-ex/0607050, doi:10.1103/PhysRevD.74.071101.

[131] R. Aaij, et al., Evidence for the decay $X(3872) \rightarrow \psi(2 S) \gamma$, Nucl. Phys. B886 (2014) 665-680. arXiv:1404.0275, doi:10.1016/j.nuclphysb.2014.06.011. 
[132] K. Abe, et al., Evidence for $X(3872) \rightarrow \gamma J / \psi$ and the sub-threshold decay $X(3872) \rightarrow \omega J / \psi,[129]$. arXiv:hep-ex/0505037.

[133] G. Gokhroo, et al., Observation of a near-threshold $D^{0} \overline{D^{0}} \pi^{0}$ enhancement in $B \rightarrow D^{0} \overline{D^{0}} \pi^{0} K$ decay, Phys. Rev. Lett. 97 (2006) 162002. arXiv:hep-ex/0606055, doi:10.1103/PhysRevLett. 97.162002.

[134] T. Aushev, et al., Study of the $B \rightarrow X(3872)\left(\rightarrow D^{* 0} \overline{D^{0}}\right) K$ decay, Phys. Rev. D81 (2010) 031103. arXiv:0810.0358, doi:10.1103/PhysRevD.81.031103.

[135] M. Ablikim, et al., Precision measurements of branching fractions for $\psi^{\prime} \rightarrow \pi^{0} J \psi$ and $\eta J \psi$, Phys. Rev. D86 (2012) 092008. arXiv:1210.3746, doi:10.1103/PhysRevD.86.092008.

[136] K. Terasaki, $\omega-\rho^{0}$ mixing as a possible origin of the hypothetical isospin non-conservation in the $X(3872) \rightarrow \pi^{+} \pi^{-} J / \psi$ decay, Prog. Theor. Phys. 122 (2010) 1285-1290. arXiv:0904.3368, doi:10.1143/PTP.122.1285.

[137] Yu. S. Kalashnikova, Coupled-channel model for charmonium levels and an option for $X(3872)$, Phys. Rev. D72 (2005) 034010. arXiv:hep-ph/0506270, doi:10.1103/PhysRevD.72.034010.

[138] M. B. Voloshin, Channel coupling in $e^{+} e^{-}$annihilation into heavy meson pairs at the $D^{*} \bar{D}^{*}$ thresholdarXiv:hep-ph/0602233.

[139] I. V. Danilkin, Yu. A. Simonov, Channel coupling in heavy quarkonia: Energy levels, mixing, widths and new states, Phys. Rev. D81 (2010) 074027. arXiv:0907.1088, doi:10.1103/ PhysRevD.81.074027.

[140] T. Barnes, E. S. Swanson, Hadron loops: General theorems and application to charmonium, Phys. Rev. C77 (2008) 055206. arXiv:0711.2080, doi:10.1103/PhysRevC.77.055206.

[141] M. R. Pennington, D. J. Wilson, Decay channels and charmonium mass-shifts, Phys. Rev. D76 (2007) 077502. arXiv:0704.3384, doi:10.1103/PhysRevD.76.077502.

[142] C. Meng, K.-T. Chao, Decays of the X(3872) and $\chi_{c 1}(2 \mathrm{P})$ charmonium, Phys. Rev. D75 (2007) 114002. arXiv:hep-ph/0703205, doi:10.1103/PhysRevD.75.114002.

[143] B. Aubert, et al., Study of resonances in exclusive $B$ Decays to $\bar{D}^{(*)} D^{(*)} K$, Phys. Rev. D77 (2008) 011102. arXiv:0708.1565, doi:10.1103/PhysRevD.77.011102.

[144] L. Maiani, A. D. Polosa, V. Riquer, Indications of a four-quark structure for the $X(3872)$ and $X(3876)$ particles from recent Belle and BABAR data, Phys. Rev. Lett. 99 (2007) 182003. arXiv: 0707.3354, doi:10.1103/PhysRevLett.99.182003.

[145] H. Albrecht, et al., Search for Hadronic $b \rightarrow u$ Decays, Phys. Lett. B241 (1990) 278-282. doi: 10.1016/0370-2693(90)91293-K.

[146] C. Hanhart, Yu. S. Kalashnikova, A. E. Kudryavtsev, A. V. Nefediev, Reconciling the X(3872) with the near-threshold enhancement in the $D^{0} \bar{D}^{* 0}$ final state, Phys. Rev. D76 (2007) 034007. arXiv:0704.0605, doi:10.1103/PhysRevD.76.034007.

[147] V. Bhardwaj, et al., Observation of $X(3872) \rightarrow J / \psi \gamma$ and search for $X(3872) \rightarrow \psi^{\prime} \gamma$ in B decays, Phys. Rev. Lett. 107 (2011) 091803. arXiv:1105.0177, doi:10.1103/PhysRevLett.107.091803. 
[148] E. S. Swanson, Molecular interpretation of the $X(3872)$, in: Proceedings, 32nd International Conference on High Energy Physics (ICHEP 2004): Beijing, China, August 16-22, 2004, 2004, pp. 1037-1039. arXiv:hep-ph/0410284.

[149] Y. Dong, A. Faessler, T. Gutsche, V. E. Lyubovitskij, $J / \psi \gamma$ and $\psi(2 S) \gamma$ decay modes of the X(3872), J. Phys. G38 (2011) 015001. arXiv:0909.0380, doi:10.1088/0954-3899/38/1/ 015001 .

[150] J. Ferretti, G. Galat, E. Santopinto, Quark structure of the $X(3872)$ and $\chi_{b}(3 P)$ resonances, Phys. Rev. D90 (5) (2014) 054010. arXiv:1401.4431, doi:10.1103/PhysRevD.90.054010.

[151] T. Barnes, S. Godfrey, Charmonium options for the X(3872), Phys. Rev. D69 (2004) 054008. arXiv:hep-ph/0311162, doi:10.1103/PhysRevD.69.054008.

[152] B.-Q. Li, K.-T. Chao, Higher charmonia and $X, Y, Z$ states with screened potential, Phys. Rev. D79 (2009) 094004. arXiv:0903.5506, doi:10.1103/PhysRevD.79.094004.

[153] T. A. Lahde, Exchange current operators and electromagnetic dipole transitions in heavy quarkonia, Nucl. Phys. A714 (2003) 183-212. arXiv:hep-ph/0208110, doi:10.1016/S0375-9474(02) 01362-3.

[154] A. M. Badalian, V. D. Orlovsky, Yu. A. Simonov, B. L. G. Bakker, The ratio of decay widths of $X(3872)$ to $\psi^{\prime} \gamma$ and $J / \psi \gamma$ as a test of the $X(3872)$ dynamical structure, Phys. Rev. D85 (2012) 114002. arXiv:1202.4882, doi:10.1103/PhysRevD.85.114002.

[155] T. Mehen, R. Springer, Radiative decays $X(3872) \rightarrow \psi(2 S) \gamma$ and $\psi(4040) \rightarrow X(3872) \gamma$ in effective field theory, Phys. Rev. D83 (2011) 094009. arXiv:1101.5175, doi:10.1103/PhysRevD.83. 094009 .

[156] T.-H. Wang, G.-L. Wang, Radiative E1 decays of X(3872), Phys. Lett. B697 (2011) 233-237. arXiv:1006.3363, doi:10.1016/j.physletb.2011.02.014.

[157] E. J. Eichten, K. Lane, C. Quigg, New states above charm threshold, Phys. Rev. D73 (2006) 014014, [Erratum: Phys. Rev.D73,079903(2006)]. arXiv:hep-ph/0511179, doi:10.1103/ PhysRevD.73.014014,10.1103/PhysRevD.73.079903.

[158] B. Aubert, et al., Observation of $Y(3940) \rightarrow J / \psi \omega$ in $B \rightarrow J / \psi \omega K$ at BaBar, Phys. Rev. Lett. 101 (2008) 082001. arXiv:0711.2047, doi:10.1103/PhysRevLett.101.082001.

[159] P. del Amo Sanchez, et al., Evidence for the decay $X(3872) \rightarrow J / \psi \omega$, Phys. Rev. D82 (2010) 011101. arXiv:1005.5190, doi:10.1103/PhysRevD.82.011101.

[160] S. Uehara, et al., Observation of a charmonium-like enhancement in the $\gamma \gamma \rightarrow \omega J / \psi$ process, Phys. Rev. Lett. 104 (2010) 092001. arXiv:0912.4451, doi:10.1103/PhysRevLett.104.092001.

[161] J. P. Lees, et al., Study of $X(3915) \rightarrow J / \psi \omega$ in two-photon collisions, Phys. Rev. D86 (2012) 072002. arXiv:1207.2651, doi:10.1103/PhysRevD.86.072002.

[162] I. Adachi, et al., Study of $X(3872)$ in $B$ meson decays, in: Proceedings, 34th International Conference on High Energy Physics (ICHEP 2008): Philadelphia, Pennsylvania, July 30-August 5, 2008, 2008. arXiv:0809.1224.

URL https://inspirehep.net/record/795806/files/arXiv:0809.1224.pdf 
[163] B. Aubert, et al., Ambiguity-free measurement of $\cos (2 \beta)$ : Time-integrated and time-dependent angular analyses of $B \rightarrow J / \psi K \pi$, Phys. Rev. D71 (2005) 032005. arXiv:hep-ex/0411016, doi:10.1103/PhysRevD.71.032005.

[164] N. Soni, et al., Measurement of branching fractions for $B \rightarrow \chi_{c 1(2)} K^{(*)}$ at Belle, Phys. Lett. B634 (2006) 155-164. arXiv:hep-ex/0508032, doi:10.1016/j.physletb.2006.01.013.

[165] E. S. Swanson, The new heavy mesons: a status report, Phys. Rept. 429 (2006) 243-305. arXiv: hep-ph/0601110, doi:10.1016/j.physrep.2006.04.003.

[166] K. Abe, et al., Experimental constraints on the possible $J^{P C}$ quantum numbers of the $X(3872)$, in: Lepton and photon interactions at high energies. Proceedings, 22nd International Symposium, LP 2005, Uppsala, Sweden, June 30-July 5, 2005, 2005. arXiv: hep-ex/0505038.

[167] A. Abulencia, et al., Analysis of the quantum numbers $J^{P C}$ of the $X(3872)$, Phys. Rev. Lett. 98 (2007) 132002. arXiv:hep-ex/0612053, doi:10.1103/PhysRevLett.98.132002.

[168] V. Bhardwaj, et al., Evidence of a new narrow resonance decaying to $\chi_{c 1} \gamma$ in $B \rightarrow \chi_{c 1} \gamma K$, Phys. Rev. Lett. 111 (3) (2013) 032001. arXiv:1304.3975, doi:10.1103/PhysRevLett.111.032001.

[169] J. L. Rosner, Angular distributions in $J / \psi\left(\rho^{0}, \omega\right)$ states near threshold, Phys. Rev. D70 (2004) 094023. arXiv:hep-ph/0408334, doi:10.1103/PhysRevD.70.094023.

[170] D. V. Bugg, The X(3872) and the 3941-MeV peak in $\omega J / \psi$, Phys. Rev. D71 (2005) 016006. arXiv:hep-ph/0410168, doi:10.1103/PhysRevD.71.016006.

[171] T. Barnes, S. Godfrey, E. S. Swanson, Higher charmonia, Phys. Rev. D72 (2005) 054026. arXiv: hep-ph/0505002, doi:10.1103/PhysRevD.72.054026.

[172] V. Bhardwaj, et al., Inclusive and exclusive measurements of $B$ decays to $\chi_{c 1}$ and $\chi_{c 2}$ at Belle, Phys. Rev. D93 (5) (2016) 052016. arXiv:1512.02672, doi:10.1103/PhysRevD.93.052016.

[173] N. A. Törnqvist, Isospin breaking of the narrow charmonium state of Belle at $3872-\mathrm{MeV}$ as a deuson, Phys. Lett. B590 (2004) 209-215. arXiv:hep-ph/0402237, doi:10.1016/j.physletb. 2004.03.077.

[174] G. Y. Chen, J. P. Ma, Production of X(3872) at PANDA, Phys. Rev. D77 (2008) 097501. arXiv: 0802.2982, doi:10.1103/PhysRevD.77.097501.

[175] J. P. Lees, et al., Measurement of the $D^{*}(2010)^{+}$meson width and the $D^{*}(2010)^{+}-D^{0}$ mass difference, Phys. Rev. Lett. 111 (11) (2013) 111801. arXiv:1304.5657, doi:10.1103/PhysRevLett. 111.111801,10.1103/PhysRevLett.111.169902.

[176] E. Braaten, An estimate of the partial width for X(3872) into pp , Phys. Rev. D77 (2008) 034019. arXiv:0711.1854, doi:10.1103/PhysRevD.77.034019.

[177] L. Maiani, V. Riquer, F. Piccinini, A. D. Polosa, Four quark interpretation of Y(4260), Phys. Rev. D72 (2005) 031502. arXiv:hep-ph/0507062, doi:10.1103/PhysRevD.72.031502.

[178] K. Terasaki, A new tetra-quark interpretation of X(3872), Prog. Theor. Phys. 118 (2007) 821-826. arXiv:0706.3944, doi:10.1143/PTP.118.821.

[179] B. Aubert, et al., Search for a charged partner of the $X(3872)$ in the $B$ meson decay $B \rightarrow$ $X^{-} K, X^{-} \rightarrow J / \psi \pi^{-} \pi^{0}$, Phys. Rev. D71 (2005) 031501. arXiv:hep-ex/0412051, doi:10.1103/ PhysRevD.71.031501. 
[180] B. Aubert, et al., Observation of the decay $B \rightarrow J / \psi \eta K$ and search for $X(3872) \rightarrow J / \psi \eta$, Phys. Rev. Lett. 93 (2004) 041801. arXiv:hep-ex/0402025, doi:10.1103/PhysRevLett.93.041801.

[181] T. Iwashita, et al., Measurement of branching fractions for $B \rightarrow J / \psi \eta K$ decays and search for a narrow resonance in the $J / \psi \eta$ final state, PTEP 2014 (4) (2014) 043C01. arXiv:1310.2704, doi:10.1093/ptep/ptu043.

[182] E. P. Wigner, On the behavior of cross sections near thresholds, Phys. Rev. 73 (1948) 1002-1009. doi:10.1103/PhysRev.73.1002.

[183] D. V. Bugg, The covalent bond in particle spectroscopy, AIP Conf. Proc. 1257 (2010) 303-305. arXiv:1001.1712, doi:10.1063/1.3483338.

[184] G. S. Bali, S. Collins, C. Ehmann, Charmonium spectroscopy and mixing with light quark and open charm states from $n_{F}=2$ lattice QCD, Phys. Rev. D84 (2011) 094506. arXiv:1110.2381, doi:10.1103/PhysRevD.84.094506.

[185] K. Abe, et al., Observation of a near-threshold $\omega J / \psi$ mass enhancement in exclusive $B \rightarrow K \omega J / \psi$ decays, Phys. Rev. Lett. 94 (2005) 182002. arXiv:hep-ex/0408126, doi:10.1103/PhysRevLett. 94.182002.

[186] K. Abe, et al., Observation of a new charmonium state in double charmonium production in $e^{+} e^{-}$ annihilation at $\sqrt{s} \approx 10.6 \mathrm{GeV}$, Phys. Rev. Lett. 98 (2007) 082001. arXiv:hep-ex/0507019, doi:10.1103/PhysRevLett.98.082001.

[187] P. Pakhlov, et al., Production of new charmonium like states in $e^{+} e^{-} \rightarrow J / \psi D^{(*)} \bar{D}^{(*)}$ at $\sqrt{s} \approx 10.6$ GeV, Phys. Rev. Lett. 100 (2008) 202001. arXiv:0708.3812, doi:10.1103/PhysRevLett.100. 202001.

[188] B. Aubert, et al., Observation of a broad structure in the $\pi^{+} \pi^{-} J / \psi$ mass spectrum around 4.26-GeV/c ${ }^{2}$, Phys. Rev. Lett. 95 (2005) 142001. arXiv:hep-ex/0506081, doi:10.1103/ PhysRevLett.95.142001.

[189] K. Abe, et al., Study of the $Y(4260)$ resonance in $e^{+} e^{-}$collisions with initial state radiation at Belle, in: Proceedings of the 33rd International Conference on High Energy Physics (ICHEP '06): Moscow, Russia, July 26-August 2, 2006, 2006. arXiv:hep-ex/0612006.

[190] B. Aubert, et al., Study of the $\pi^{+} \pi^{-} J / \psi$ mass spectrum via initial-state radiation at BaBar, in: Proceedings, 34th International Conference on High Energy Physics (ICHEP 2008): Philadelphia, Pennsylvania, July 30-August 5, 2008, 2008. arXiv:0808.1543.

URL http://www-public.slac.stanford.edu/sciDoc/docMeta.aspx?slacPubNumber= slac-pub-13360

[191] J. P. Lees, et al., Study of the reaction $e^{+} e^{-} \rightarrow J / \psi \pi^{+} \pi^{-}$via initial-state radiation at BaBar, Phys. Rev. D86 (2012) 051102. arXiv:1204.2158, doi:10.1103/PhysRevD.86.051102.

[192] C. Z. Yuan, et al., Measurement of $e^{+} e^{-} \rightarrow \pi^{+} \pi^{-} J / \psi$ cross-section via initial state radiation at Belle, Phys. Rev. Lett. 99 (2007) 182004. arXiv:0707.2541, doi:10.1103/PhysRevLett.99. 182004.

[193] X. L. Wang, et al., Observation of two resonant structures in $e^{+} e^{-} \rightarrow \pi^{+} \pi^{-} \psi(2 S)$ via initial state radiation at Belle, Phys. Rev. Lett. 99 (2007) 142002. arXiv:0707.3699, doi:10.1103/ PhysRevLett .99.142002. 
[194] B. Aubert, et al., Evidence of a broad structure at an invariant mass of $4.32 \mathrm{GeV} / \mathrm{c}^{2}$ in the reaction $e^{+} e^{-} \rightarrow \pi^{+} \pi^{-} \psi(2 S)$ measured at BaBar, Phys. Rev. Lett. 98 (2007) 212001. arXiv: hep-ex/0610057, doi:10.1103/PhysRevLett.98.212001.

[195] Q. He, et al., Confirmation of the Y(4260) resonance production in ISR, Phys. Rev. D74 (2006) 091104. arXiv:hep-ex/0611021, doi:10.1103/PhysRevD.74.091104.

[196] T. E. Coan, et al., Charmonium decays of $Y(4260), \psi(4160)$ and $\psi(4040)$, Phys. Rev. Lett. 96 (2006) 162003. arXiv:hep-ex/0602034, doi:10.1103/PhysRevLett.96.162003.

[197] Q. Wang, C. Hanhart, Q. Zhao, Decoding the riddle of $Y(4260)$ and $Z_{c}(3900)$, Phys. Rev. Lett. 111 (13) (2013) 132003. arXiv:1303.6355, doi:10.1103/PhysRevLett.111.132003.

[198] C. Z. Yuan, et al., Observation of $e^{+} e^{-} \rightarrow K^{+} K^{-} J / \psi$ via initial state radiation at Belle, Phys. Rev. D77 (2008) 011105. arXiv:0709.2565, doi:10.1103/PhysRevD.77.011105.

[199] B. Aubert, et al., Study of $J / \psi \pi^{+} \pi^{-}$states produced in $B^{0} \rightarrow J / \psi \pi^{+} \pi^{-} K^{0}$ and $B^{-} \rightarrow$ $J / \psi \pi^{+} \pi^{-} K^{-}$, Phys. Rev. D73 (2006) 011101. arXiv:hep-ex/0507090, doi:10.1103/PhysRevD. 73.011101.

[200] E. van Beveren, G. Rupp, The $X(4260)$ and possible confirmation of $\psi(3 D), \psi(5 S), \psi(4 D), \psi(6 S)$, and $\psi(5 D)$ in $J / \psi \pi^{+} \pi^{-}$arXiv:0904.4351.

[201] M. Ablikim, et al., Determination of the $\psi(3770), \psi(4040), \psi(4160)$ and $\psi(4415)$ resonance parameters, eConf C070805 (2007) 02, [Phys. Lett.B660,315(2008)]. arXiv:0705.4500, doi: $10.1016 / j \cdot$ physletb.2007.11.100.

[202] Q. Wang, C. Hanhart, Q. Zhao, Decoding the riddle of $Y(4260)$ and $Z_{c}(3900)$, Phys. Rev. Lett. 111 (13) (2013) 132003. arXiv:1303.6355, doi:10.1103/PhysRevLett.111.132003.

[203] M. Ablikim, et al., Evidence of two resonant structures in $e^{+} e^{-} \rightarrow \pi^{+} \pi^{-} h_{c}$, Phys. Rev. Lett. 118 (9) (2017) 092002. arXiv:1610.07044, doi:10.1103/PhysRevLett.118.092002.

[204] G. Pakhlova, et al., Observation of a near-threshold enhancement in the $e^{+} e^{-} \rightarrow \Lambda_{c}^{+} \Lambda_{c}^{-}$cross section using initial-state radiation, Phys. Rev. Lett. 101 (2008) 172001. arXiv:0807.4458, doi: 10.1103/PhysRevLett.101.172001.

[205] S. K. Choi, et al., Observation of a resonance-like structure in the $\pi^{ \pm} \psi^{\prime}$ mass distribution in exclusive $B \rightarrow K \pi^{ \pm} \psi^{\prime}$ decays, Phys. Rev. Lett. 100 (2008) 142001. arXiv:0708.1790, doi: 10.1103/PhysRevLett.100.142001.

[206] R. Mizuk, et al., Observation of two resonance-like structures in the $\pi^{+} \chi_{c 1}$ mass distribution in exclusive $\overline{B^{0}} \rightarrow K^{-} \pi^{+} \chi_{c 1}$ decays, Phys. Rev. D78 (2008) 072004. arXiv:0806.4098, doi: 10.1103/PhysRevD.78.072004.

[207] R. Mizuk, et al., Dalitz analysis of $B \rightarrow K \pi^{+} \psi^{\prime}$ decays and the $Z(4430)^{+}$, Phys. Rev. D80 (2009) 031104. arXiv:0905.2869, doi:10.1103/PhysRevD.80.031104.

[208] K. Chilikin, et al., Observation of a new charged charmonium like state in $\bar{B}^{0} \rightarrow J / \psi K^{-} \pi^{+}$decays, Phys. Rev. D90 (11) (2014) 112009. arXiv:1408.6457, doi:10.1103/PhysRevD.90.112009.

[209] R. Aaij, et al., Observation of the resonant character of the $Z(4430)^{-}$state, Phys. Rev. Lett. 112 (22) (2014) 222002. arXiv:1404.1903, doi:10.1103/PhysRevLett.112.222002. 
[210] J. P. Lees, et al., Search for the $Z_{1}(4050)^{+}$and $Z_{2}(4250)^{+}$states in $\bar{B}^{0} \rightarrow \chi_{c 1} K^{-} \pi^{+}$and $B^{+} \rightarrow \chi_{c 1} K_{S}^{0} \pi^{+}$, Phys. Rev. D85 (2012) 052003. arXiv:1111.5919, doi:10.1103/PhysRevD. 85.052003.

[211] M. Ablikim, et al., Observation of a charged charmonium like structure in $e^{+} e^{-} \rightarrow \pi^{+} \pi^{-} J / \psi$ at $\sqrt{s}$ $=4.26 \mathrm{GeV}$, Phys. Rev. Lett. 110 (2013) 252001. arXiv:1303.5949, doi:10.1103/PhysRevLett. 110.252001 .

[212] Z. Q. Liu, et al., Study of $e^{+} e^{-} \rightarrow \pi^{+} \pi^{-} J / \psi$ and observation of a charged charmonium like state at Belle, Phys. Rev. Lett. 110 (2013) 252002. arXiv:1304.0121, doi:10.1103/PhysRevLett. 110.252002 .

[213] T. Xiao, S. Dobbs, A. Tomaradze, K. K. Seth, Observation of the charged hadron $Z_{c}^{ \pm}(3900)$ and evidence for the neutral $Z_{c}^{0}(3900)$ in $e^{+} e^{-} \rightarrow \pi \pi J / \psi$ at $\sqrt{s}=4170 \mathrm{MeV}$, Phys. Lett. B727 (2013) 366-370. arXiv:1304.3036, doi:10.1016/j.physletb.2013.10.041.

[214] M. Ablikim, et al., Confirmation of a charged charmoniumlike state $Z_{c}(3885)^{\mp}$ in $e^{+} e^{-} \rightarrow$ $\pi^{ \pm}\left(D \bar{D}^{*}\right)^{\mp}$ with double $D$ tag, Phys. Rev. D92 (9) (2015) 092006. arXiv:1509.01398, doi: 10.1103/PhysRevD.92.092006.

[215] M. Ablikim, et al., Observation of a charged charmoniumlike structure in $e^{+} e^{-} \rightarrow\left(D^{*} \bar{D}^{*}\right)^{ \pm} \pi^{\mp}$ at $\sqrt{s}=4.26 \mathrm{GeV}$, Phys. Rev. Lett. 112 (13) (2014) 132001. arXiv:1308.2760, doi:10.1103/ PhysRevLett.112.132001.

[216] M. Ablikim, et al., Observation of a charged charmonium like structure $Z_{c}(4020)$ and Search for the $Z_{c}(3900)$ in $e^{+} e^{-} \rightarrow \pi^{+} \pi^{-} h_{c}$, Phys. Rev. Lett. 111 (24) (2013) 242001. arXiv:1309.1896, doi:10.1103/PhysRevLett.111.242001.

[217] A. Ali, C. Hambrock, W. Wang, Tetraquark interpretation of the charged bottomonium-like states $Z_{b}^{+-}(10610)$ and $Z_{b}^{+-}(10650)$ and implications, Phys. Rev. D85 (2012) 054011. arXiv:1110.1333, doi:10.1103/PhysRevD.85.054011.

[218] M. Ablikim, et al., Observation of a charged $\left(D \bar{D}^{*}\right)^{ \pm}$mass peak in $e^{+} e^{-} \rightarrow \pi D \bar{D}^{*}$ at $\sqrt{s}=4.26$ GeV, Phys. Rev. Lett. 112 (2) (2014) 022001. arXiv:1310.1163, doi:10.1103/PhysRevLett. 112.022001 .

[219] M. Ablikim, et al., Observation of $Z_{c}(3900)^{0}$ in $e^{+} e^{-} \rightarrow \pi^{0} \pi^{0} J / \psi$, Phys. Rev. Lett. 115 (11) (2015) 112003. arXiv:1506.06018, doi:10.1103/PhysRevLett.115.112003.

[220] M. Ablikim, et al., Observation of a neutral structure near the $D \bar{D}^{*}$ mass threshold in $e^{+} e^{-} \rightarrow$ $\left(D \bar{D}^{*}\right)^{0} \pi^{0}$ at $\sqrt{s}=4.226$ and $4.257 \mathrm{GeV}$, Phys. Rev. Lett. 115 (22) (2015) 222002. arXiv: 1509.05620, doi:10.1103/PhysRevLett.115.222002.

[221] M. Ablikim, et al., Observation of $e^{+} e^{-} \rightarrow \pi^{0} \pi^{0} h_{c}$ and a neutral charmonium like structure $Z_{c}(4020)^{0}$, Phys. Rev. Lett. 113 (21) (2014) 212002. arXiv:1409.6577, doi:10.1103/ PhysRevLett.113.212002.

[222] M. Ablikim, et al., Observation of a neutral charmoniumlike state $Z_{c}(4025)^{0}$ in $e^{+} e^{-} \rightarrow$ $\left(D^{*} \bar{D}^{*}\right)^{0} \pi^{0}$, Phys. Rev. Lett. 115 (18) (2015) 182002. arXiv:1507.02404, doi:10.1103/ PhysRevLett.115.182002.

[223] J. Bian, A review of heavy exotic states, arXiv:1411.4343. 
[224] A. Bondar, et al., Observation of two charged bottomonium-like resonances in Y(5S) decays, Phys. Rev. Lett. 108 (2012) 122001. arXiv:1110.2251, doi:10.1103/PhysRevLett.108.122001.

[225] D. Ebert, R. N. Faustov, V. O. Galkin, Properties of heavy quarkonia and $B_{c}$ mesons in the relativistic quark model, Phys. Rev. D67 (2003) 014027. arXiv:hep-ph/0210381, doi:10.1103/ PhysRevD.67.014027.

[226] I. V. Danilkin, V. D. Orlovsky, Yu. A. Simonov, Hadron interaction with heavy quarkonia, Phys. Rev. D85 (2012) 034012. arXiv:1106.1552, doi:10.1103/PhysRevD.85.034012.

[227] D. V. Bugg, An explanation of Belle states $Z_{b}(10610)$ and $Z_{b}(10650)$, Europhys. Lett. 96 (2011) 11002. arXiv:1105.5492, doi:10.1209/0295-5075/96/11002.

[228] M. Karliner, H. J. Lipkin, Possibility of exotic states in the $\Upsilon$ system, arXiv:0802.0649.

[229] A. E. Bondar, A. Garmash, A. I. Milstein, R. Mizuk, M. B. Voloshin, Heavy quark spin structure in $Z_{b}$ resonances, Phys. Rev. D84 (2011) 054010. arXiv:1105.4473, doi:10.1103/PhysRevD. 84.054010 .

[230] K. F. Chen, et al., Observation of anomalous $\Upsilon(1 S) \pi^{+} \pi^{-}$and $\Upsilon(2 S) \pi^{+} \pi^{-}$production near the $\Upsilon(5 S)$ resonance, Phys. Rev. Lett. 100 (2008) 112001. arXiv:0710.2577, doi:10.1103/ PhysRevLett.100.112001.

[231] A. Garmash, et al., Observation of Zb(10610) and Zb(10650) Decaying to B Mesons, Phys. Rev. Lett. 116 (21) (2016) 212001. arXiv:1512.07419, doi:10.1103/PhysRevLett.116.212001.

[232] P. Krokovny, et al., First observation of the $Z \frac{0}{b}(10610)$ in a Dalitz analysis of $\Upsilon(10860)$ $\rightarrow \Upsilon(\mathrm{nS}) \pi^{0} \pi^{0}$, Phys.Rev. D88 (2013) 052016. arXiv:1308.2646, doi:10.1103/PhysRevD.88. 052016.

[233] T. Aaltonen, et al., Evidence for a narrow near-threshold structure in the $J / \psi \phi$ mass spectrum in $B^{+} \rightarrow J / \psi \phi K^{+}$decays, Phys. Rev. Lett. 102 (2009) 242002. arXiv:0903.2229, doi:10.1103/ PhysRevLett.102.242002.

[234] V. M. Abazov, et al., Search for the $X(4140)$ in $B^{+} \rightarrow J / \psi \phi K^{+}$decays with the D0 detector, Phys. Rev. D89 (1) (2014) 012004. arXiv:1309.6580, doi:10.1103/PhysRevD.89.012004.

[235] S. Chatrchyan, et al., Observation of a peaking structure in the $J / \psi \phi$ mass spectrum from $B^{ \pm} \rightarrow J / \psi \phi K^{ \pm}$decays, Phys. Lett. B734 (2014) 261-281. arXiv:1309.6920, doi:10.1016/ j.physletb.2014.05.055.

[236] J. P. Lees, et al., Study of $B^{ \pm, 0} \rightarrow J / \psi K^{+} K^{-} K^{ \pm, 0}$ and search for $B^{0} \rightarrow J / \psi \phi$ at BaBar, Phys. Rev. D91 (1) (2015) 012003. arXiv:1407.7244, doi:10.1103/PhysRevD.91.012003.

[237] R. Aaij, et al., Search for the $X(4140)$ state in $B^{+} \rightarrow J / \psi \phi K^{+}$decays, Phys. Rev. D85 (2012) 091103. arXiv:1202.5087, doi:10.1103/PhysRevD.85.091103.

[238] S. Cheng-Ping, XYZ particles at Belle, Chin. Phys. C34 (2010) 615-620. arXiv:0912.2386, doi:10.1088/1674-1137/34/6/001.

[239] R. Aaij, et al., Observation of $J / \psi \phi$ structures consistent with exotic states from amplitude analysis of $B^{+} \rightarrow J / \psi \phi K^{+}$decays, Phys. Rev. Lett. 118 (2) (2017) 022003. arXiv:1606.07895, doi:10.1103/PhysRevLett.118.022003. 
[240] T. Aaltonen, et al., Observation of the $Y(4140)$ structure in the $J / \psi \phi$ mass spectrum in $B^{ \pm} \rightarrow$ $J / \psi \phi K$ decaysarXiv:1101.6058.

[241] R. Aaij, et al., Amplitude analysis of $B^{+} \rightarrow J / \psi \phi K^{+}$decays, Phys. Rev. D95 (1) (2017) 012002. arXiv:1606.07898, doi:10.1103/PhysRevD.95.012002.

[242] C. Weisser, M. Williams, Machine learning and multivariate goodness of fitarXiv:1612.07186.

[243] E. S. Swanson, Cusps and exotic charmonia, Int. J. Mod. Phys. E25 (07) (2016) 1642010. arXiv: 1504.07952, doi:10.1142/S0218301316420106.

[244] X. Liu, The hidden charm decay of Y(4140) by the rescattering mechanism, Phys. Lett. B680 (2009) 137-140. arXiv:0904.0136, doi:10.1016/j.physletb.2009.08.049.

[245] M. Karliner, J. L. Rosner, Exotic resonances due to $\eta$ exchange, Nucl. Phys. A954 (2016) 365-370. arXiv:1601.00565, doi:10.1016/j.nuclphysa.2016.03.057.

[246] X. Chen, X. Lü, R. Shi, X. Guo, Mass of Y(4140) in Bethe-Salpeter equation for quarks, arXiv: 1512.06483 .

[247] Z.-G. Wang, Z.-C. Liu, X.-H. Zhang, Analysis of the $Y(4140)$ and related molecular states with QCD sum rules, Eur. Phys. J. C64 (2009) 373-386. arXiv:0907.1467, doi:10.1140/epjc/ s10052-009-1156-2.

[248] X. Liu, H.-W. Ke, The line shape of the radiative open-charm decay of $Y(4140)$ and $Y(3930)$, Phys. Rev. D80 (2009) 034009. arXiv:0907.1349, doi:10.1103/PhysRevD.80.034009.

[249] J.-R. Zhang, M.-Q. Huang, $(Q \bar{s})^{(*)}(\bar{Q} s)^{(*)}$ molecular states from QCD sum rules: a view on $Y(4140)$, J. Phys. G37 (2010) 025005. arXiv:0905.4178, doi:10.1088/0954-3899/37/2/ 025005 .

[250] G.-J. Ding, Possible molecular states of $D_{s}^{*}$ anti- $D_{s}^{*}$ system and $Y(4140)$, Eur. Phys. J. C64 (2009) 297-308. arXiv:0904.1782, doi:10.1140/epjc/s10052-009-1146-4.

[251] R. M. Albuquerque, M. E. Bracco, M. Nielsen, A QCD sum rule calculation for the $Y(4140)$ narrow structure, Phys. Lett. B678 (2009) 186-190. arXiv:0903.5540, doi:10.1016/j.physletb. 2009. 06.022 .

[252] T. Branz, T. Gutsche, V. E. Lyubovitskij, Hadronic molecule structure of the $Y(3940)$ and Y(4140), Phys. Rev. D80 (2009) 054019. arXiv:0903.5424, doi:10.1103/PhysRevD.80.054019.

[253] X. Liu, S.-L. Zhu, Y(4143) is probably a molecular partner of $Y(3930)$, Phys. Rev. D80 (2009) 017502, [Erratum: Phys. Rev.D85,019902(2012)]. arXiv:0903.2529, doi:10.1103/PhysRevD. 85.019902, 10.1103/PhysRevD.80.017502.

[254] R. F. Lebed, A. D. Polosa, $\chi_{c 0}(3915)$ as the lightest $c \bar{c} s \bar{s}$ state, Phys. Rev. D93 (9) (2016) 094024. arXiv:1602.08421, doi:10.1103/PhysRevD.93.094024.

[255] V. V. Anisovich, M. A. Matveev, A. V. Sarantsev, A. N. Semenova, Exotic mesons with hidden charm as diquark-antidiquark states, Int. J. Mod. Phys. A30 (32) (2015) 1550186. arXiv:1507. 07232, doi:10.1142/S0217751X15501869.

[256] Z.-g. Wang, Y.-f. Tian, Tetraquark state candidates: Y(4140), Y(4274) and X(4350), Int. J. Mod. Phys. A30 (2015) 1550004. arXiv:1502.04619, doi:10.1142/S0217751X15500049. 
[257] N. V. Drenska, R. Faccini, A. D. Polosa, Exotic hadrons with hidden charm and strangeness, Phys. Rev. D79 (2009) 077502. arXiv:0902.2803, doi:10.1103/PhysRevD.79.077502.

[258] F. Stancu, Can $Y(4140)$ be a $c \bar{c} s \bar{s}$ tetraquark?, J. Phys. G37 (2010) 075017. arXiv:0906.2485, doi:10.1088/0954-3899/37/7/075017.

[259] N. Mahajan, Y(4140): Possible options, Phys. Lett. B679 (2009) 228-230. arXiv:0903.3107, doi:10.1016/j.physletb.2009.07.043.

[260] Z.-G. Wang, Analysis of the $Y(4140)$ with QCD sum rules, Eur. Phys. J. C63 (2009) 115-122. arXiv:0903.5200, doi:10.1140/epjc/s10052-009-1097-9.

[261] L. Maiani, A. D. Polosa, V. Riquer, Interpretation of axial resonances in $J / \psi \phi$ at LHCb, Phys. Rev. D94 (5) (2016) 054026. arXiv:1607.02405, doi:10.1103/PhysRevD.94.054026.

[262] C. P. Shen, et al., Evidence for a new resonance and search for the $Y(4140)$ in the $\gamma \gamma \rightarrow \phi J / \psi$ process, Phys. Rev. Lett. 104 (2010) 112004. arXiv:0912.2383, doi:10.1103/PhysRevLett. 104.112004.

[263] G. 't Hooft, G. Isidori, L. Maiani, A. D. Polosa, V. Riquer, A Theory of Scalar Mesons, Phys. Lett. B662 (2008) 424-430. arXiv:0801.2288, doi:10.1016/j.physletb.2008.03.036.

[264] A. H. Fariborz, R. Jora, J. Schechter, Note on a sigma model connection with instanton dynamics, Phys. Rev. D77 (2008) 094004. arXiv:0801.2552, doi:10.1103/PhysRevD.77.094004.

[265] A. A. Belavin, A. M. Polyakov, A. S. Schwartz, Yu. S. Tyupkin, Pseudoparticle Solutions of the Yang-Mills Equations, Phys. Lett. B59 (1975) 85-87. doi:10.1016/0370-2693(75)90163-X.

[266] M. A. Shifman, A. I. Vainshtein, V. I. Zakharov, Instanton Density in a Theory with Massless Quarks, Nucl. Phys. B163 (1980) 46-56. doi:10.1016/0550-3213(80)90389-2.

[267] G. 't Hooft, How Instantons Solve the U(1) Problem, Phys. Rept. 142 (1986) 357-387. doi: 10.1016/0370-1573(86)90117-1.

[268] G. 't Hooft, The Physics of instantons in the pseudoscalar and vector meson mixingarXiv:hep-th/ 9903189.

[269] D. Black, A. H. Fariborz, J. Schechter, Mechanism for a next-to-lowest lying scalar meson nonet, Phys. Rev. D61 (2000) 074001. arXiv:hep-ph/9907516, doi:10.1103/PhysRevD.61.074001.

[270] E. Swanson, $Z_{b}$ and $Z_{c}$ exotic states as coupled channel cusps, Phys.Rev. D91 (2015) 034009. arXiv:1409.3291, doi:10.1103/PhysRevD.91.034009.

[271] D. V. Bugg, How resonances can synchronise with thresholds, J. Phys. G35 (2008) 075005. arXiv: 0802.0934, doi:10.1088/0954-3899/35/7/075005.

[272] E. Braaten, How the $Z(3900)$ reveals the spectra of quarkonium hybrid and tetraquark mesons, Phys. Rev. Lett. 111 (2013) 162003. arXiv:1305.6905, doi:10.1103/PhysRevLett.111.162003.

[273] F. E. Close, P. R. Page, The Production and decay of hybrid mesons by flux tube breaking, Nucl. Phys. B443 (1995) 233-254. arXiv:hep-ph/9411301, doi:10.1016/0550-3213(95)00085-7.

[274] J. J. Dudek, The lightest hybrid meson supermultiplet in QCD, Phys. Rev. D84 (2011) 074023. arXiv:1106.5515, doi:10.1103/PhysRevD.84.074023. 
[275] S. M. Ryan, Hadron spectroscopy from lattice quantum chromodynamics, EPJ Web Conf. 130 (2016) 01002. doi:10.1051/epjconf/201613001002.

[276] E. Kou, O. Pene, Suppressed decay into open charm for the Y(4260) being an hybrid, Phys. Lett. B631 (2005) 164-169. arXiv:hep-ph/0507119, doi:10.1016/j.physletb.2005.09.013.

[277] S.-L. Zhu, The Possible interpretations of Y(4260), Phys. Lett. B625 (2005) 212. arXiv:hep-ph/ 0507025, doi:10.1016/j.physletb.2005.08.068.

[278] C. A. Meyer, E. S. Swanson, Hybrid mesons, Prog. Part. Nucl. Phys. 82 (2015) 21-58. arXiv: 1502.07276, doi:10.1016/j.ppnp.2015.03.001.

[279] M. R. Pennington, Impressions of the meson spectrum: hybrids \& exotics, present and future, EPJ Web Conf. 113 (2016) 01014. arXiv:1509.02555, doi:10.1051/epjconf/201611301014.

[280] M. Berwein, N. Brambilla, J. Tarrs Castell, A. Vairo, Quarkonium hybrids with nonrelativistic effective field theories, Phys. Rev. D92 (11) (2015) 114019. arXiv:1510.04299, doi:10.1103/ PhysRevD.92.114019.

[281] H. Al Ghoul, et al., First results from the GlueX experiment, AIP Conf. Proc. 1735 (2016) 020001. arXiv:1512.03699, doi:10.1063/1.4949369.

[282] M. F. M. Lutz, et al., Physics performance report for PANDA: strong interaction studies with antiprotons, arXiv:0903.3905.

[283] M. Cleven, F.-K. Guo, C. Hanhart, U.-G. Meißner, Bound state nature of the exotic $Z_{b}$ states, Eur. Phys. J. A47 (2011) 120. arXiv:1107.0254, doi:10.1140/epja/i2011-11120-6.

[284] F. E. Close, P. R. Page, The $D^{* 0} \bar{D}^{0}$ threshold resonance, Phys. Lett. B578 (2004) 119-123. arXiv:hep-ph/0309253, doi:10.1016/j.physletb.2003.10.032.

[285] E. S. Swanson, The new heavy mesons: A status report, Phys. Rept. 429 (2006) 243-305. arXiv: hep-ph/0601110, doi:10.1016/j.physrep.2006.04.003.

[286] M. Cleven, Q. Wang, F.-K. Guo, C. Hanhart, U.-G. Meißner, Q. Zhao, Confirming the molecular nature of the $Z_{b}(10610)$ and the $Z_{b}(10650)$, Phys. Rev. D87 (7) (2013) 074006. arXiv:1301.6461, doi:10.1103/PhysRevD.87.074006.

[287] C. Bignamini, B. Grinstein, F. Piccinini, A. D. Polosa, C. Sabelli, Is the $X(3872)$ production cross section at Tevatron compatible with a hadron molecule interpretation?, Phys. Rev. Lett. 103 (2009) 162001. arXiv:0906.0882, doi:10.1103/PhysRevLett.103.162001.

[288] P. Artoisenet, E. Braaten, Production of the $X(3872)$ at the Tevatron and the LHC, Phys. Rev. D81 (2010) 114018. arXiv:0911.2016, doi:10.1103/PhysRevD.81.114018.

[289] T. Gutsche, M. Kesenheimer, V. E. Lyubovitskij, Radiative and dilepton decays of the hadronic molecule $Z_{c}^{+}(3900)$, Phys. Rev. D90 (9) (2014) 094013. arXiv:1410.0259, doi:10.1103/ PhysRevD.90.094013.

[290] M. Cleven, Systematic study of hadronic molecules in the heavy-quark sector, Ph.D. thesis, Bonn U. (2013). arXiv:1405.4195.

URL https://inspirehep.net/record/1296844/files/arXiv:1405.4195.pdf 
[291] F. K. Guo, C. Hanhart, Yu. S. Kalashnikova, P. Matuschek, R. V. Mizuk, A. V. Nefediev, Q. Wang, J. L. Wynen, Interplay of quark and meson degrees of freedom in near-threshold states: A practical parametrization for line shapes, Phys. Rev. D93 (7) (2016) 074031. arXiv:1602.00940, doi: 10.1103/PhysRevD.93.074031.

[292] P. Artoisenet, E. Braaten, D. Kang, Using line shapes to discriminate between binding mechanisms for the X(3872), Phys. Rev. D82 (2010) 014013. arXiv:1005.2167, doi:10.1103/PhysRevD.82. 014013.

[293] C. Hanhart, Yu. S. Kalashnikova, A. V. Nefediev, Interplay of quark and meson degrees of freedom in a near-threshold resonance: multi-channel case, Eur. Phys. J. A47 (2011) 101-110. arXiv: 1106.1185, doi:10.1140/epja/i2011-11101-9.

[294] C. Meng, J. J. Sanz-Cillero, M. Shi, D.-L. Yao, H.-Q. Zheng, Refined analysis on the X(3872) resonance, Phys. Rev. D92 (3) (2015) 034020. arXiv:1411.3106, doi:10.1103/PhysRevD.92. 034020 .

[295] T. Barnes, F. E. Close, E. S. Swanson, Molecular interpretation of the supercharmonium state Z(4475), Phys. Rev. D91 (1) (2015) 014004. arXiv:1409.6651, doi:10.1103/PhysRevD.91. 014004 .

[296] S. Weinberg, Tetraquark mesons in large $N$ quantum chromodynamics, Phys. Rev. Lett. 110 (2013) 261601. arXiv:1303.0342, doi:10.1103/PhysRevLett.110.261601.

[297] M. Knecht, S. Peris, Narrow tetraquarks at large N, Phys. Rev. D88 (2013) 036016. arXiv: 1307.1273, doi:10.1103/PhysRevD.88.036016.

[298] G. Rossi, G. Veneziano, The string-junction picture of multiquark states: an update, JHEP 06 (2016) 041. arXiv:1603.05830, doi:10.1007/JHEP06(2016)041.

[299] A. Hosaka, T. Iijima, K. Miyabayashi, Y. Sakai, S. Yasui, Exotic hadrons with heavy flavors: $X, Y, Z$, and related states, PTEP 2016 (6) (2016) 062C01. arXiv:1603.09229, doi:10.1093/ ptep/ptw045.

[300] C. DeTar, LQCD: Flavor Physics and Spectroscopy, PoS Lepton Photon 2015 (2016) 023. arXiv: 1511.06884 .

[301] Y. Chen, et al., Low-energy scattering of the $\left(D \bar{D}^{*}\right)^{ \pm}$system and the resonance-like structure $Z_{c}(3900)$, Phys. Rev. D89 (9) (2014) 094506. arXiv:1403.1318, doi:10.1103/PhysRevD.89. 094506 .

[302] L. Maiani, F. Piccinini, A. D. Polosa, V. Riquer, The Z(4430) and a New Paradigm for Spin Interactions in Tetraquarks, Phys. Rev. D89 (2014) 114010. arXiv:1405.1551, doi:10.1103/ PhysRevD .89.114010.

[303] A. Ali, C. Hambrock, I. Ahmed, M. J. Aslam, A case for hidden $b \bar{b}$ tetraquarks based on $e^{+} e^{-} \rightarrow b \bar{b}$ cross section between $\sqrt{s}=10.54$ and $11.20 \mathrm{GeV}$, Phys. Lett. B684 (2010) 28-39. arXiv:0911. 2787, doi:10.1016/j.physletb.2009.12.053.

[304] A. Ali, C. Hambrock, M. J. Aslam, A tetraquark interpretation of the BELLE data on the anomalous $\Upsilon(1 S) \pi^{+} \pi^{-}$and $\Upsilon(2 S) \pi^{+} \pi^{-}$production near the $\Upsilon(5 S)$ resonance, Phys. Rev. Lett. 104 (2010) 162001, [Erratum: Phys. Rev. Lett.107,049903(2011)]. arXiv:0912.5016, doi:10. 1103/PhysRevLett.104.162001,10.1103/PhysRevLett.107.049903. 
[305] A. Ali, C. Hambrock, S. Mishima, Tetraquark-based analysis and predictions of the cross sections and distributions for the processes $e^{+} e^{-} \rightarrow \Upsilon(1 S)\left(\pi^{+} \pi^{-}, K^{+} K^{-}, \eta \pi^{0}\right)$ near $\Upsilon(5 S)$, Phys. Rev. Lett. 106 (2011) 092002. arXiv:1011.4856, doi:10.1103/PhysRevLett.106.092002.

[306] R. A. Briceno, et al., Issues and opportunities in exotic hadrons, Chin. Phys. C40 (4) (2016) 042001. arXiv:1511.06779, doi:10.1088/1674-1137/40/4/042001.

[307] R. L. Jaffe, Exotica, Phys. Rept. 409 (2005) 1-45. arXiv:hep-ph/0409065, doi:10.1016/j. physrep.2004.11.005.

[308] C. Alexandrou, P. de Forcrand, B. Lucini, Evidence for diquarks in lattice QCD, Phys. Rev. Lett. 97 (2006) 222002. arXiv:hep-lat/0609004, doi:10.1103/PhysRevLett.97.222002.

[309] A. V. Manohar, M. B. Wise, Heavy quark physics, Camb. Monogr. Part. Phys. Nucl. Phys. Cosmol. 10 (2000) 1-191.

[310] M. Karliner, S. Nussinov, J. L. Rosner, $Q Q \bar{Q} \bar{Q}$ states: masses, production, and decays, Phys. Rev. D95 (3) (2017) 034011. arXiv:1611.00348, doi:10.1103/PhysRevD.95.034011.

[311] A. Ali, L. Maiani, A. D. Polosa, V. Riquer, Hidden-beauty charged tetraquarks and heavy quark spin conservation, Phys. Rev. D91 (1) (2015) 017502. arXiv:1412.2049, doi:10.1103/ PhysRevD.91.017502.

[312] D. Ebert, R. N. Faustov, V. O. Galkin, Masses of heavy tetraquarks in the relativistic quark model, Phys. Lett. B634 (2006) 214-219. arXiv:hep-ph/0512230, doi:10.1016/j.physletb. 2006.01 .026 .

[313] M. Nielsen, F. S. Navarra, S. H. Lee, New charmonium states in QCD sum rules: a concise review, Phys. Rept. 497 (2010) 41-83. arXiv:0911.1958, doi:10.1016/j.physrep.2010.07.005.

[314] R. Albuquerque, S. Narison, F. Fanomezana, A. Rabemananjara, D. Rabetiarivony, G. Randriamanatrika, $X Y Z$-like spectra from Laplace sum rule at N2LO in the chiral limit, Int. J. Mod. Phys. A31 (36) (2016) 1650196. arXiv:1609.03351, doi:10.1142/S0217751X16501967.

[315] A. Ali, L. Maiani, A. D. Polosa, V. Riquer, $B_{c}^{ \pm}$decays into tetraquarks, Phys. Rev. D94 (3) (2016) 034036. arXiv:1604.01731, doi:10.1103/PhysRevD.94.034036.

[316] D. Santel, et al., Measurements of the $\Upsilon(10860)$ and $\Upsilon(11020)$ resonances via $\sigma\left(e^{+} e^{-} \rightarrow\right.$ $\Upsilon(n S) \pi^{+} \pi^{-}$), Phys. Rev. D93 (1) (2016) 011101. arXiv:1501.01137, doi:10.1103/PhysRevD. 93.011101.

[317] A. E. Bondar, A. Garmash, A. I. Milstein, R. Mizuk, M. B. Voloshin, Heavy quark spin structure in $Z_{b}$ resonances, Phys. Rev. D84 (2011) 054010. arXiv:1105.4473, doi:10.1103/PhysRevD. 84.054010 .

[318] A. Ali, W. Wang, Production of the exotic $1^{--}$hadrons $\phi(2170), X(4260)$ and $Y_{b}(10890)$ at the LHC and Tevatron via the Drell-Yan mechanism, Phys. Rev. Lett. 106 (2011) 192001. arXiv: 1103.4587, doi:10.1103/PhysRevLett.106.192001.

[319] B. Aubert, et al., A structure at $2175 \mathrm{MeV}$ in $e^{+} e^{-} \rightarrow \phi f_{0}(980)$ observed via initial-state radiation, Phys. Rev. D74 (2006) 091103. arXiv:hep-ex/0610018, doi:10.1103/PhysRevD.74.091103.

[320] M. Ablikim, et al., Observation of $Y(2175)$ in $J / \psi \rightarrow \eta \phi f_{0}(980)$, Phys. Rev. Lett. 100 (2008) 102003. arXiv:0712.1143, doi:10.1103/PhysRevLett.100.102003. 
[321] C. P. Shen, et al., Observation of the $\phi(1680)$ and the $Y(2175)$ in $e^{+} e^{-} \rightarrow \phi \pi^{+} \pi^{-}$, Phys. Rev. D80 (2009) 031101. arXiv:0808.0006, doi:10.1103/PhysRevD.80.031101.

[322] N. V. Drenska, R. Faccini, A. D. Polosa, Higher tetraquark particles, Phys. Lett. B669 (2008) 160-166. arXiv:0807.0593, doi:10.1016/j.physletb.2008.09.038.

[323] T. J. Burns, Phenomenology of $P_{c}(4380)^{+}, P_{c}(4450)^{+}$and related states, Eur. Phys. J. A51 (11) (2015) 152. arXiv:1509.02460, doi:10.1140/epja/i2015-15152-6.

[324] F.-K. Guo, U.-G. Meißner, W. Wang, Z. Yang, How to reveal the exotic nature of the $P_{c}(4450)$, Phys. Rev. D92 (7) (2015) 071502. arXiv:1507.04950, doi:10.1103/PhysRevD.92.071502.

[325] X.-H. Liu, Q. Wang, Q. Zhao, Understanding the newly observed heavy pentaquark candidates, Phys. Lett. B757 (2016) 231-236. arXiv:1507.05359, doi:10.1016/j.physletb.2016.03.089.

[326] M. Mikhasenko, A triangle singularity and the LHCb pentaquarks, sarXiv:1507.06552.

[327] U.-G. Meißner, J. A. Oller, Testing the $\chi_{c 1} p$ composite nature of the $P_{c}(4450)$, Phys. Lett. B751 (2015) 59-62. arXiv:1507.07478, doi:10.1016/j.physletb.2015.10.015.

[328] H.-X. Chen, W. Chen, X. Liu, T. G. Steele, S.-L. Zhu, Towards exotic hidden-charm pentaquarks in QCD, Phys. Rev. Lett. 115 (17) (2015) 172001. arXiv:1507.03717, doi:10.1103/ PhysRevLett.115.172001.

[329] J. He, $\bar{D} \Sigma_{c}^{*}$ and $\bar{D}^{*} \Sigma_{c}$ interactions and the LHCb hidden-charmed pentaquarks, Phys. Lett. B753 (2016) 547-551. arXiv:1507.05200, doi:10.1016/j.physletb.2015.12.071.

[330] L. Roca, J. Nieves, E. Oset, LHCb pentaquark as a $\bar{D}^{*} \Sigma_{c}-\bar{D}^{*} \Sigma_{c}^{*}$ molecular state, Phys. Rev. D92 (9) (2015) 094003. arXiv: 1507.04249, doi:10.1103/PhysRevD.92.094003.

[331] R. Chen, X. Liu, X.-Q. Li, S.-L. Zhu, Identifying exotic hidden-charm pentaquarks, Phys. Rev. Lett. 115 (13) (2015) 132002. arXiv:1507.03704, doi:10.1103/PhysRevLett.115.132002.

[332] C. W. Xiao, U. G. Meißner, $J / \psi\left(\eta_{c}\right) N$ and $\Upsilon\left(\eta_{b}\right) N$ cross sections, Phys. Rev. D92 (11) (2015) 114002. arXiv:1508.00924, doi:10.1103/PhysRevD.92.114002.

[333] G.-N. Li, X.-G. He, M. He, Some predictions of diquark model for hidden charm pentaquark discovered at the LHCb, JHEP 12 (2015) 128. arXiv:1507.08252, doi:10.1007/JHEP12(2015) 128 .

[334] A. Mironov, A. Morozov, Is the pentaquark doublet a hadronic molecule?, JETP Lett. 102 (5) (2015) 271-273, [Pisma Zh. Eksp. Teor. Fiz.102,no.5,302(2015)]. arXiv:1507.04694, doi:10. 7868/S0370274X15170038, 10.1134/S0021364015170099.

[335] V. V. Anisovich, M. A. Matveev, J. Nyiri, A. V. Sarantsev, A. N. Semenova, Pentaquarks and resonances in the $p J / \psi$ spectrum, arXiv:1507.07652.

[336] R. Ghosh, A. Bhattacharya, B. Chakrabarti, The masses of $P_{c}^{*}(4380)$ and $P_{c}^{*}(4450)$ in the quasi particle diquark mode arXiv:1508.00356.

[337] Z.-G. Wang, Analysis of $P_{c}(4380)$ and $P_{c}(4450)$ as pentaquark states in the diquark model with QCD sum rules, Eur. Phys. J. C76 (2) (2016) 70. arXiv:1508.01468, doi:10.1140/epjc/ s10052-016-3920-4. 
[338] Z.-G. Wang, T. Huang, Analysis of the $\frac{1}{2}^{ \pm}$pentaquark states in the diquark model with QCD sum rules, Eur. Phys. J. C76 (1) (2016) 43. arXiv:1508.04189, doi:10.1140/epjc/ s10052-016-3880-8.

[339] A. Ali, I. Ahmed, M. J. Aslam, A. Rehman, Heavy quark symmetry and weak decays of the b-baryons in pentaquarks with a $c \bar{c}$ component, Phys. Rev. D94 (5) (2016) 054001. arXiv: 1607.00987, doi:10.1103/PhysRevD.94.054001.

[340] R. F. Lebed, The pentaquark candidates in the dynamical diquark picture, Phys. Lett. B749 (2015) 454-457. arXiv:1507.05867, doi:10.1016/j.physletb.2015.08.032.

[341] R. Zhu, C.-F. Qiao, Pentaquark states in a diquark-triquark model, Phys. Lett. B756 (2016) 259-264. arXiv:1510.08693, doi:10.1016/j.physletb.2016.03.022.

[342] R. Aaij, et al., Evidence for exotic hadron contributions to $\Lambda_{b}^{0} \rightarrow J / \psi p \pi^{-}$decays, Phys. Rev. Lett. 117 (8) (2016) 082003, [Addendum: Phys. Rev. Lett.118,119901(2017)]. arXiv: 1606.06999, doi:10.1103/PhysRevLett.118.119901,10.1103/PhysRevLett.117.082003, 10. 1103/PhysRevLett.117.109902.

[343] L. Maiani, A. D. Polosa, V. Riquer, From pentaquarks to dibaryons in $\Lambda_{b}(5620)$ decays, Phys. Lett. B750 (2015) 37-38. arXiv:1508.04459, doi:10.1016/j.physletb.2015.08.049. 